\title{
WestVirginiaUniversity
}

THE RESEARCH REPOSITORY @ WVU

Graduate Theses, Dissertations, and Problem Reports

2011

\section{Modeling of Roughness and Dissipation of Actuated Polymer Films}

Joaquin Maria Gutierrez

West Virginia University

Follow this and additional works at: https://researchrepository.wvu.edu/etd

\section{Recommended Citation}

Gutierrez, Joaquin Maria, "Modeling of Roughness and Dissipation of Actuated Polymer Films" (2011). Graduate Theses, Dissertations, and Problem Reports. 4726.

https://researchrepository.wvu.edu/etd/4726

This Thesis is protected by copyright and/or related rights. It has been brought to you by the The Research Repository @ WVU with permission from the rights-holder(s). You are free to use this Thesis in any way that is permitted by the copyright and related rights legislation that applies to your use. For other uses you must obtain permission from the rights-holder(s) directly, unless additional rights are indicated by a Creative Commons license in the record and/ or on the work itself. This Thesis has been accepted for inclusion in WVU Graduate Theses, Dissertations, and Problem Reports collection by an authorized administrator of The Research Repository @ WVU. For more information, please contact researchrepository@mail.wvu.edu. 


\title{
Modeling of Roughness and Dissipation of Actuated Polymer Films
}

\author{
by \\ Joaquín María Gutiérrez \\ Thesis submitted to the \\ College of Engineering and Mineral Resources \\ at West Virginia University \\ in partial fulfillment of the requirements \\ for the degree of \\ Master of Science
in
Mechanical Engineering
}

Ever J. Barbero, Ph.D., Chair

Darran R. Cairns, Ph.D.

Victor H. Mucino, Ph.D.

Department of Mechanical and Aerospace Engineering

Morgantown, West Virginia

2011

Keywords: Dissipation, Actuated Polymer Films, Finite Element, ABAQUS ${ }^{\circledR}$, MATLAB $^{\circledR}$, Hyperelasticity, Viscoelasticity, Bergstrom - Boyce Model 


\section{Abstract \\ Modeling of Roughness and Dissipation of Actuated Polymer Films}

\section{Joaquin M. Gutierrez}

Actuated polymer films (APF) are a novel kind of material with several possible applications (e.g., dynamic roughness for aerodynamic flow control, tactile responsive touch panels, dust mitigation systems for solar panels and other devices, and tunable hydrophobicity surfaces). The development of the required analytical and numerical tools for the design and analysis of APF's is addressed in this research.

A hyperelastic hysteretic material model (Bergstrom-Boyce) is used to model the APF. A program to calibrate the Bergstrom-Boyce material parameters from experimental data is developed using MATLAB ${ }^{\circledR}$. The soundness of this calibration tool is assessed using experimental data from the literature.

To calculate the response of the APF and the power requirement to actuate the film, a program is written. The performance of this program is validated with experimental results from the literature. Two- and three-dimensional models are analyzed and compared using ABAQUS ${ }^{\circledR}$.

A procedure for the determination of the volume fraction of the actuators embedded in the APF is proposed using digital image processing techniques and Voronoi diagrams. 


\section{Dedication}

To my wife, Sofia. 


\section{Acknowledgments}

I owe my deepest gratitude to my advisor, Dr. Ever J. Barbero. He has done an excellent job guiding me through this thesis, from the very first stages of the research until the review of this manuscript. I also would like to thank to the members of the examination committee, Dr. Darran R. Cairns and Dr. Victor H. Mucino.

I appreciate the help from Nick Morris, who supplied the pictures of the actuated polymer film samples used in Chapter 5. I am also grateful to my friends Fritz Campo and Joaquín Fernández Bugna who helped me many times.

I would like to thank to my family, who have always supported me. Lastly, I truly appreciate the support and love of my wife, Sofia. 


\section{Contents}

$\begin{array}{lll}1 & \text { Introduction } & 1\end{array}$

1.1 Background . . . . . . . . . . . . . . . . . . . . . . . . 1

1.2 Literature Review . . . . . . . . . . . . . . . . . . . . . . 2

1.3 Thesis Outline . . . . . . . . . . . . . . . . . . . . . . . 3

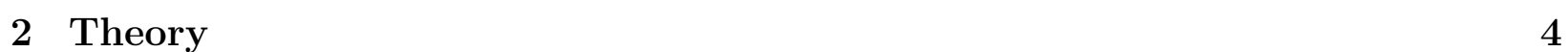

2.1 Hvperelastic Materials . . . . . . . . . . . . . . . . . 4

2.1.1 Classification of Hyperelastic Models . . . . . . . . . . . . . . . 4

2.2 Mullins Effect . . . . . . . . . . . . . . . . . . . . 5

2.3 True Stress - True Strain . . . . . . . . . . . . . . . . . . . 5

2.4 Finite Deformation Kinematics $\ldots \ldots \ldots$

2.5 Stress Measures . . . . . . . . . . . . . . . . . . . . . . . 8

2.6 Bergström - Bovce Material Model . . . . . . . . . . . . . . . . . 9

2.6 .1 Constitutive and Kinematic Model ... . . . . . . . . . . . . 9

2.7 Uniform Uniaxial Deformation and Incompressible Material . . . . . . . . . . 12

2.7 .1 Application to the BB Model . . . . . . . . . . . . . . . . . 15

$\begin{array}{llr}3 & \text { BB Model Parameters Fit } & 17\end{array}$

3.1 Program Structure . . . . . . . . . . . . . . . . . . . . . . . . . 17

3.1 .1 Main Program . . . . . . . . . . . . . . . . . . . . . 17

3.1 .2 Constitutive Equation Function . . . . . . . . . . . . . . . . . . . 19

3.1 .3 Error Function . . . . . . . . . . . . . . . . . . . . . . . . . . . . 19

3.2 Examples . . . . . . . . . . . . . . . . . . . . 20

\begin{tabular}{|lll}
4 & Finite Element Analysis & $\mathbf{2 4}$
\end{tabular}

4.1 Introduction . . . . . . . . . . . . . . . . . . . . . . . . . . . . . 24

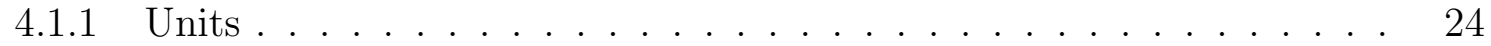

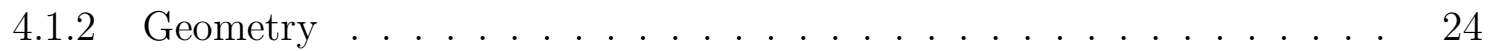

4.1 .3 Material Models and Properties . . . . . . . . . . . . . . . . . . . 24

4.1 .4 Hvbrid Elements . . . . . . . . . . . . . . . . . . . . . 27

4.1 .5 Periodic Boundarv Conditions . . . . . . . . . . . . . . . . . . 27

$4.1 .6 \quad$ Geometric Non-Linearity . . . . . . . . . . . . . . . . . . . . . . . . 28

4.1 .7 Post-Processing . . . . . . . . . . . . . . . . . . . . . . . . . 28

4.2 Models to Compare with the Laboratory Experiments . . . . . . . . . . . . . 28

4.2 .1 Geometry . . . . . . . . . . . . . . . . . . . . . . . . . 28 
$4.2 .2 \quad$ Material Properties . . . . . . . . . . . . . . . . . . . . . . . . . . . . . . . 29

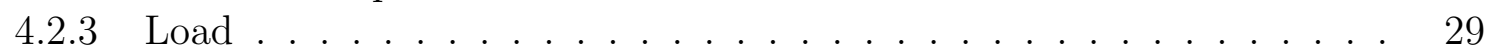

4.2 .4 Contact $\ldots \ldots \ldots \ldots \ldots$

4.2 .5 Mesh . . . . . . . . . . . . . . . . . . . . . . . 30

4.2 .6 Partially Constrained Top Glass Model . . . . . . . . . . . . . . . . 31

4.2 .7 Totally Constrained Top Glass Model . . . . . . . . . . . . . . . . . . 33

4.3 Models of the Application . . . . . . . . . . . . . . . . . . . . . . 36

4.3 .1 Material Properties . . . . . . . . . . . . . . . . . . . . . . . . . . . . 37

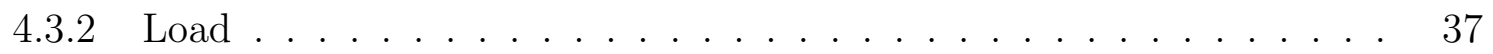

4.3 .3 Two-Dimensional Diluted Model . . . . . . . . . . . . . . . . . . 37

4.3.4 Three-Dimensional Diluted Model . . . . . . . . . . . . . . . . . . . . 39

4.3 .5 Correlation between 2D and 3D . . . . . . . . . . . . . 42

4.3 .6 Two Dimensional - Parametric Analysis . . . . . . . . . . . . . . 44

5 Volume Fraction Calculation $\quad 56$

5.1 Introduction . . . . . . . . . . . . . . . . . . . . . 56

5.2 Program Structure . . . . . . . . . . . . . . . . . . . . . . . . 56

5.2 .1 Image Processing . . . . . . . . . . . . . . . . . . . . . 56

5.2 .2 Computational Geometry $\ldots \ldots \ldots$

5.2 .3 Area Fraction $\ldots \ldots \ldots \ldots$

5.2 .4 Volume Fraction . . . . . . . . . . . . . . . . . . . . . 63

5.2 .5 RVE dimensions . . . . . . . . . . . . . . . . . . . . . . . . . . . . 63

5.3 Results . . . . . . . . . . . . . . . . . . . . . . 64

6 Conclusions and Future Work $\quad 65$

6.1 Future Work . . . . . . . . . . . . . . . . . . . . . . . . 65

6.1 .1 Temperature Dependence . . . . . . . . . . . . . . . . . 65

6.1 .2 Torque Measurement . . . . . . . . . . . . . . . . . . . . . . . 65

6.1 .3 Base film mechanical properties . . . . . . . . . . . . . . . . 66

6.2 Conclusions . . . . . . . . . . . . . . . . . . . . 67

\begin{tabular}{|ll}
\hline A Code of the program to fit the BB model & $\mathbf{7 0}$
\end{tabular}

A.1 Main Program . . . . . . . . . . . . . . . . . . . . . 70

A.2 Constitutive Equation Function $\ldots \ldots \ldots \ldots \ldots$. . . . . . . . . 72

A.3 Error Function . . . . . . . . . . . . . . . . . . . . . . . . . 75

\begin{tabular}{lr}
\hline B Dissipation Calculation & 79
\end{tabular}

B.1 Comparison . . . . . . . . . . . . . . . . . . . . . . . . . . 79

B.2 PYTHON Script . . . . . . . . . . . . . . . . . . . . . . . . . . . . . . 82

B.3 Code Listing . . . . . . . . . . . . . . . . . . . . . . . . . . 83

\begin{tabular}{|l|l|l|l}
\hline C Relationship between the two- and three-dimensional loads & 85
\end{tabular}

C.1 Three-dimensional case . . . . . . . . . . . . . . . . . . . . . . . . . 8 85

C.2 Two-dimensional case . . . . . . . . . . . . . . . . . . . . . . . . 86

\begin{tabular}{lr}
\hline D Minimum RVE depth & 88
\end{tabular} 
\begin{tabular}{ll}
\hline E ABAQUS Scripts & $\mathbf{9 0}$
\end{tabular} E.1 Contact Model - Partially Constrained Top Glass . . . . . . . . . . . . . . . 90

E.2 Two-Dimensional Model . . . . . . . . . . . . . . . . . . . . . . 103

E.3 Three-Dimensional Model . . . . . . . . . . . . . . . . . . . . . . . 121

\begin{tabular}{|lll}
\hline F Code of Volume Fraction Calculation Script & 135
\end{tabular}

\begin{tabular}{ll}
\hline G Companion CD Contents & 143
\end{tabular}

\begin{tabular}{lr}
\hline Bibliography & 144
\end{tabular} 


\section{List of Figures}

$2.1 \quad$ BB Model Scheme $\quad \ldots \ldots \ldots \ldots$

2.2 Uniaxial Deformation . . . . . . . . . . . . . . . . . . . . . . . . 13

3.1 Acrvlate-Butadiene Rubber (ABR) . . . . . . . . . . . . . . . 20

3.2 Nitrile Rubber . . . . . . . . . . . . . . . . . . . . . . . . . . . . . . . . . . . 22

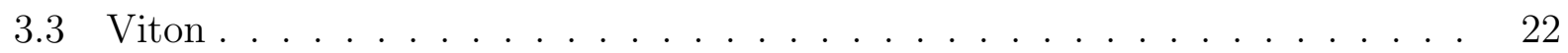

3.4 Silicone Rubber . . . . . . . . . . . . . . . . . . . . . . . . . . . . . . . 23

4.1 Stress-Strain Curves for Hvtrel 4056 (TPE) . . . . . . . . . . . . . . . . . 27

4.2 Two-Dimensional, Two-Actuators RVE Geometry . . . . . . . . . . . . . . 29

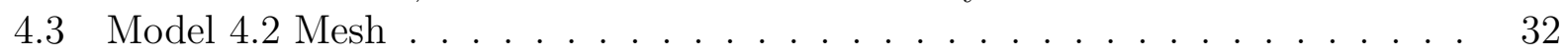

4.4 Vertical displacement of the top surface of the base film . . . . . . . . . . . 33

4.5 Vertical displacement contour plot. actuators with same polarity . . . . . . . 34

4.6 Vertical displacement contour plot. actuators with opposite polaritv . . . . 3 35

4.7 Vertical reaction of the top glass as a function of the base film compressibility 36

4.8 Two-Dimensional. One-Actuator Application RVE Geometry . . . . . . . . . 38

4.9 Svmmetrv on the RVE . . . . . . . . . . . . . . . . . . . . . . . . . 39

4.10 Three-Dimensional, One-Actuator Application RVE Geometry . . . . . . . . 39

4.11 Three-dimensional model mesh . . . . . . . . . . . . . . . . . . . 41

4.12 Surf. dissip. power $-V_{f}$ for 3D models $\ldots \ldots \ldots$. . . . . . . . 42

4.13 Dissip. power $-V_{f}$ for $3 \mathrm{D}$ models $\ldots \ldots \ldots$. . . . . . . . . 43

4.14 Ratios for roughness and surface dissipation power . . . . . . . . . . . . 43

4.15 Roughness as a function of the excitation frequencv . . . . . . . . . . . . 46

4.16 Surface Dissipation Power as a function of the excitation frequency . . . . 46

4.17 Roughness as a function of the surface traction magnitude . . . . . . . . . . 47

4.18 Surf. dissip. power as a func. of the surf. traction magnitude . . . . . . . . . 47

4.19 Roughness as a function of the actuator's vertical offset . . . . . . . . . . . 48

4.20 Surface Dissipation Power as a function of the actuator's vertical offset . . . 48

4.21 Roughness as a function of the actuator's aspect ratio . . . . . . . . . . . . 49

4.22 Surface Dissipation Power as a function of the actuator's aspect ratio . . . . 50

4.23 Roughness as a function of the volume fraction . . . . . . . . . . . . 51

4.24 Surface Dissipation Power as a function of the volume fraction . . . . . . . 51

4.25 Roughness as a function of the actuator Young's modulus . . . . . . . . . . 52

4.26 Surf. dissip. power as a func. of the actuator $\mathrm{E} \ldots \ldots \ldots$. . . . . . . . 52

4.27 Roughness as a function of the top film Young's modulus . . . . . . . . . . 53 
4.28 Surf. dissip. power as a func. of the top film E . . . . . . . . . . . . . 53

4.29 Roughness as a function of the number of elements in the mesh . . . . . . 54

4.30 Surface Dissipation Power as a function of the number of elements in the mesh 55

5.1 Image A of the actuators embedded in PVA . . . . . . . . . . . . . . 57

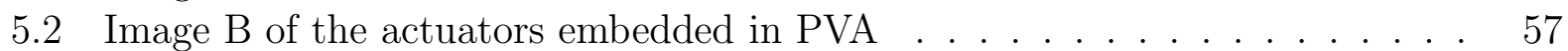

5.3 Image A converted to black and white . . . . . . . . . . . . . . . . . 58

5.4 Image B converted to black and white . . . . . . . . . . . . . . . . 59

5.5 Image A after being filled and morphologically opened and closed . . . . . . 59

5.6 Image B after being filled and morphologically opened and closed . . . . . . 60

5.7 Image A after removal of the filtered regions . . . . . . . . . . . . . . . . 61

5.8 Image B after removal of the filtered regions . . . . . . . . . . . . . . . . . 61

5.9 Image A - Actuators and their associated Voronoi cells . . . . . . . . . . . . 62

5.10 Image B - Actuators and their associated Voronoi cells . . . . . . . . . . . . 62

5.11 Image A - Bounding boxes for the interior Voronoi cells . . . . . . . . . . . . 63

5.12 Image B - Bounding boxes for the interior Voronoi cells . . . . . . . . . . . . 64

B.1 Stress-Strain curve for $\dot{\epsilon}=0.05 \ldots \ldots \ldots$. . . . . . . . . . . 81

B.2 Creep dissipation energy (ALLCD) for the whole model . . . . . . . . . . . . . 82

C.1 Three-dimensional torque differential . . . . . . . . . . . . . . . . 86

C.2 Two-dimensional torque differential . . . . . . . . . . . . . . . . . . 87

D.1 Spherical droplets become prolate spheroids when stretched . . . . . . . . . . 88

D.2 Minimum theoretical depth of the RVE . . . . . . . . . . . . . . . . . 89 


\section{List of Tables}

3.1 Parameters fit for the BB material model . . . . . . . . . . . . . . . . 21

4.1 Stress-Strain Curves for Hvtrel 4056 (TPE) . . . . . . . . . . . . . . . . 26

4.2 Two Actuators RVE Two-DimensionalGeometrv . . . . . . . . . . . . . . . . . 29

4.3 Two-Dimensional. One Actuator Application RVE Geometry . . . . . . . . . 38

4.4 Three-Dimensional. One Actuator Application RVE Geometry . . . . . . . . 39

4.5 Results for the two- and three-dimensional diluted models . . . . . . . . . . 41

4.6 Results for the two-dimensional parametric models . . . . . . . . . . . . 45

5.1 Results for the mean value $\bar{x}$ and standard deviation $s$ estimators $\ldots \ldots 64$

B.1 Vertical displacement of the top surface as a function of time . . . . . . . 80 


\section{Table of Symbols and Notation}

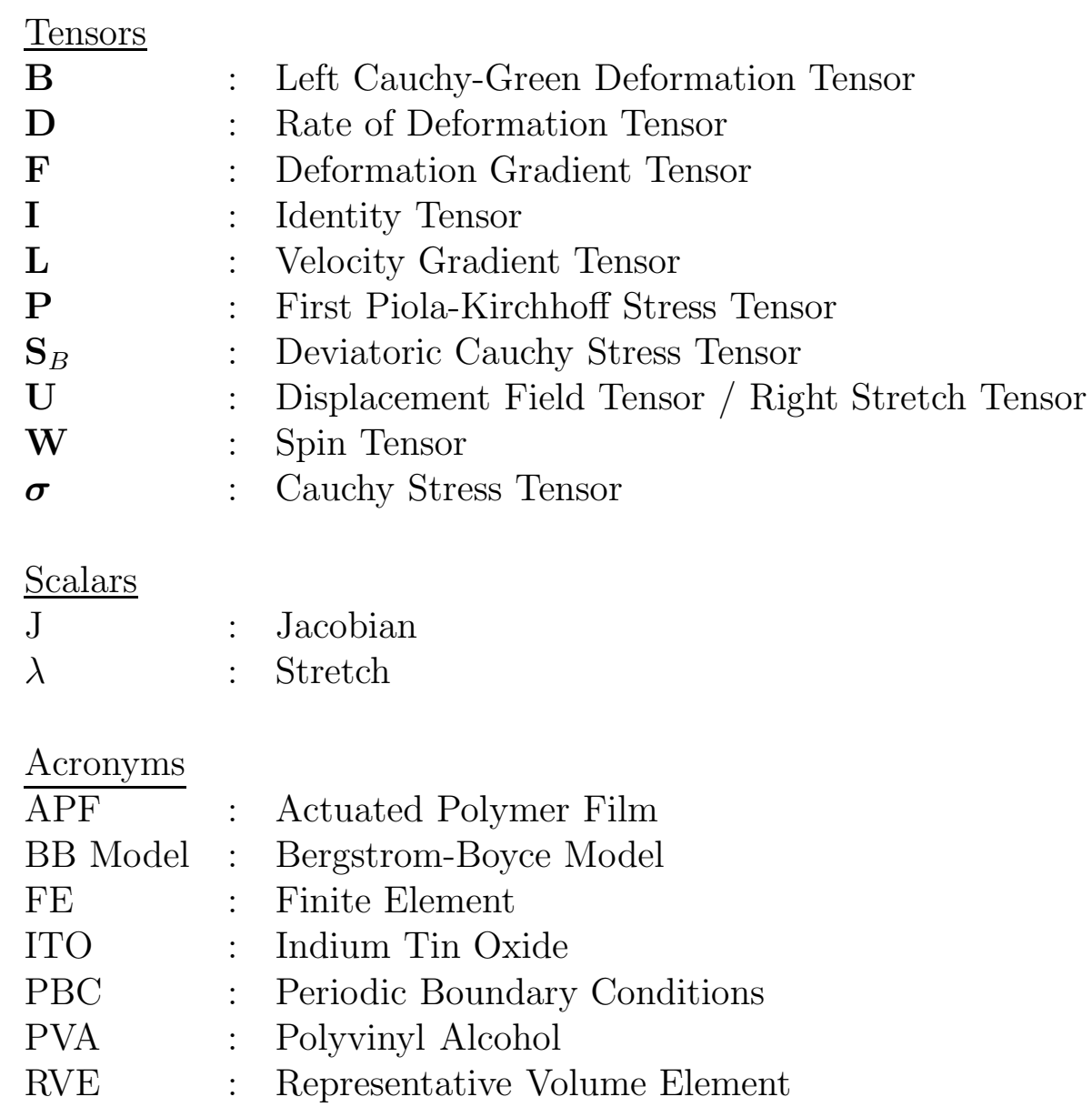

Boldface letters denote tensors. 


\section{Chapter 1}

\section{Introduction}

\section{$1.1 \quad$ Background}

The goal of the present work is to develop a set of tools for the design and analysis of actuated polymer films (APF). In the context of this work, the actuator polymer films considered are made of electrically responsive liquid crystal ellipsoids embedded in a polymeric film. When these actuators are subjected to an electric field, they try to align with the field gradient.

A possible application of these novel materials is in the aerodynamic flow control field. It has been studied that flow separation in airfoils can be reduced when the leading edge can change its roughness at specific frequencies and amplitudes (sub-boundary layer) [33, 39, 28]. Reductions in the flow separation can result in lift enhancement, drag reduction or both. A crucial factor in the feasibility of the application of dynamic roughness for flow control, is that the power savings associated with the improved aerodynamics must be greater that the power required to actuate the APF. Therefore, the ability to calculate the power consumption and the roughness achieved when the APF is actuated is of paramount importance.

Other possible applications of the actuated polymer films suggested by Morris et al. [42], include tactile responsive touch panels, dust mitigation systems for solar panels and other devices, and tunable hydrophobicity surfaces.

As it was mentioned in the previous paragraphs, it is very important to be able to calculate how much will the surface of the actuated polymer film change its geometry and at what cost, power-wise speaking. Although this could be determined with physical testing, it is of special interest to have the analytical and numerical tools to do it virtually. Since the actuated polymer film technology is new and still under development, the capacity of doing virtual testing allows to explore different scenarios in advance. For example, currently, the liquid crystal actuators are embedded in polyvinyl alcohol (PVA) [42], but because PVA is soluble in water [48, it could not be used for dynamic roughness applications in aircraft wings. Therefore, different materials (possibly rubbery elastomers) should be analyzed as possible candidates. Furthermore, many achievable geometric configurations (varying the actuator's geometry, film thickness, etc) can be tested using a parametric analysis, creating savings in time and money.

A procedure for the calculation of the power required to actuate the APF and the roughness obtained in the surface of the film are at the core of the present work. Other related 
tasks, like the fit of experimental data to a particular material model and the determination of the volume fraction of the actuators in the APF are also developed in this document.

\subsection{Literature Review}

The key component in the actuated polymer films are the actuators themselves. Responsive liquid crystal rods have been developed by Cairns et al. [21, 44]. The first potential applications sought for these actuators were active mixing in microfluidic channels and electro-rheological fluids [21].

More recently, the idea of embedding these actuators in a polymeric film was investigated by Morris et al. [42]. Currently the electric field is applied to the film when it is placed between two plates of indium-tin oxide (ITO) coated glass. For applications like aerodynamic flow control, at least one of the plates must be removed and the external surface of the APF should be coated with an electrically conductive thin layer in order to apply the voltage differential [20].

Since the possible applications of the actuated polymer films require a measurable change in the roughness of the surface, the deformations are expected to be finite. Therefore the infinitesimal theory shall not be used, and a hyperelastic model should be used.

A relatively new constitutive model for large strain, time-dependent, elastomeric materials has been developed by Bergstrom and Boyce [11, 13]. This model has the ability to capture the viscous rate-dependence and the non-linear relationship between stress and strain. This model was later slightly modified by Bergstrom and Boyce to improve the hysteretic behavior of the model under cyclic loading [16]. The Bergstrom-Boyce model has been also successfully used to model soft biological tissues [16] and filled elastomers [14, 15].

The hyperelastic model that will be used in conjunction with the Bergstrom-Boyce model in the present work is the model proposed by Arruda and Boyce [4].

In the recent years, some authors have worked on the finite element implementation of the Bergstrom-Boyce model [23, 3, 24]. The Bergstrom-Boyce model has been implemented in general purpose commercial finite element packages like ABAQUS ${ }^{\circledR}$ and ANSYS ${ }^{\circledR}$. For ABAQUS ${ }^{\circledR}$, at least until version 6.9 there is a flaw in the calculation of the dissipated energy (see Appendix $\mathrm{B}$ ). ANSYS ${ }^{\circledR}$ introduced the Bergstrom-Boyce model in the 12.0 release. The author found inconsistencies in the results from the implementation used in ANSYS ${ }^{\circledR} 12.0$, and reported this fact to the company's Technical Support [34]. Later, ANSYS ${ }^{\circledR}$ issued a Defect Analysis Report confirming the problem [1].

Johansson et al. report a calibration procedure for the Bergstrom-Boyce model using a gradient-based optimization method [35, 36] for the uniaxial stress state. In the present work, a gradient-free optimization method will be used to do the calibration of the Bergstrom-Boyce model. The gradient-free method is easier to implement because the analytical derivatives of the constitutive equation with respect to the parameters and the flow rule are not needed, but a higher computational cost must be paid, for the same accuracy in the results. 


\subsection{Thesis Outline}

In Chapter 2, the theoretical aspects that will be used in the following Chapters are developed from first principles. A brief description of some of the different hyperelastic models is given, and the necessity for such models is outlined. Some basic equations of finite kinematics are derived. The Bergstrom-Boyce model is introduced and the particular case of uniaxial deformation is analyzed.

A procedure to fit the parameters of the Bergstrom-Boyce model is developed for uniaxial loading, assuming incompressibility of the material in Chapter 3. This procedure is employed to fit experimental data for rubbery materials, available from the literature, and the goodness of fit is assessed.

Finite element models of the actuated polymer films are developed in Chapter 4. A program is written to calculate the response of the APF and the power requirement to actuate the film. The performance of this program is validated with experimental results from the literature.

Models to compare with the laboratory are analyzed in the first part of Chapter 4. In these models, the electrically conductive thin coating is not included.

Then, two- and three-dimensional models including the top film are analyzed. These models represent what the real application of the actuated polymer films (APF) would be. The power required to actuate the films is calculated and the response of the top surface, as well. The correlation of the dissipation power and roughness between the two- and threedimensional models is computed.

At the end of the Chapter 4, a parametric analysis is done on a two-dimensional model. The geometry, loading and mesh are varied to analyze their impact in the results. A mesh convergence study is also carried out.

Finally, a novel methodology to calculate the volume fraction of the actuators embedded in the APF is proposed. The input for this method is a digital image of the APF. A series of image enhancement techniques are applied to the original image and the area of each actuator is digitally measured. The domain is partitioned using Voronoi graphs and the volume fraction is statistically calculated. 


\section{Chapter 2}

\section{Theory}

\subsection{Hyperelastic Materials}

Hyperelastic materials are derived from a postulated Helmholtz free energy potential $\Psi$. If the free energy function is a function of the strain only, then it is referred as the strain energy function [38, 31].

$$
\Psi=\Psi(\mathbf{F})
$$

The formulation of hyperelastic materials is needed to describe the behavior of materials under finite deformations.

\subsubsection{Classification of Hyperelastic Models}

The hyperelastic material models can be classified in two groups,

1. Phenomenological Models

2. Mechanistic or Statistical Models

The phenomenological models describe the material behavior from a macroscopic point of view, fitting the parameters of postulated equations to experimental data [31]. Some examples of phenomenological models are Saint Venant-Kirchhoff, Odgen, Mooney-Rivlin and Yeoh.

The mechanistic or statistical models focus on the deformation mechanism and its relation with the microscopic structure of the material. Rubbery materials are made of a very large number of extremely long polymer chains. Due to the large number of possible configurations on each chain and the large number of chains, it is reasonable to use statistic methods to derive the elasticity equations for these materials [38, §8.1],[46, §3.3]. Some examples of mechanistic models are Neo-Hookean, Arruda-Boyce, Treloar, Anand and Flory. In the present work the Arruda-Boyce hyperelastic model will be used. 


\subsection{Mullins Effect}

During the first loading cycles many elastomers present a stress softening behavior. This behavior is associated with damage. After reaching a certain strain level, subsequent loading and unloading up to that strain will not cause further softening after a few cycles [31]. In the present work this phenomena is not going to be analyzed, but it is important to bear in mind that material samples should be conditioned before testing to remove this effect [11].

\subsection{True Stress - True Strain}

It is important to understand the difference between true stresses and strains and their engineering counterparts. The engineering stress is defined as the force acting per undeformed unit area. The true stress is the force acting per unit area in the deformed configuration. If the strains are infinitesimal the difference is minimal, but this is not true as the strains are finite.

The engineering strain is defined as

$$
\epsilon^{\mathrm{eng}}=\frac{\Delta L}{L}=\frac{\ell-L}{L}=\frac{\ell}{L}-1
$$

where $L$ and $\ell$ are the initial and final lengths of the fiber, respectively.

Rearranging (2.2), the stretch is defined as

$$
\lambda=\frac{\ell}{L}=\epsilon^{\mathrm{eng}}+1
$$

The true, or logarithmic, strain $\epsilon$ is obtained by integration of the incremental strain $\delta \epsilon$,

$$
\begin{aligned}
\delta \epsilon & =\frac{\delta \ell}{\ell} \\
\int \delta \epsilon & =\int_{L}^{\ell} \frac{1}{\ell} \delta \ell \\
\epsilon & =\ln (\ell)-\ln (L)=\ln \left(\frac{\ell}{L}\right)
\end{aligned}
$$

From (2.3) and (2.4),

$$
\epsilon=\ln (\lambda)=\ln \left(1+\epsilon^{\mathrm{eng}}\right)
$$

The relationship between the normal true stress and the nominal or engineering stress is given by,

$$
\sigma=\sigma^{\text {eng }} \lambda
$$

this equation will be derived at the end of Section 2.7. 


\subsection{Finite Deformation Kinematics}

The purpose of this section is to write down some basic equations of Continuum Mechanics that will be used for the development of the forthcoming sections. Some derivations are included for the sake of clarity, but by no means the intention of this chapter is to develop all the equations that are derived in any Continuum Mechanics book.

If a continuum medium is subjected to a motion field, the displacement field $\mathbf{U}$ can be represented as

$$
\mathbf{U}(\mathbf{X}, t)=\mathbf{x}(\mathbf{X}, t)-\mathbf{X}
$$

where $\mathbf{X}$ is the current position and $\mathbf{X}$ is the position in the reference configuration [31, 38]. The gradient of the the displacement field with respect $\mathbf{X}$ gives,

$$
\frac{\partial \mathbf{U}}{\partial \mathbf{X}}=\frac{\partial \mathbf{x}}{\partial \mathbf{X}}-\mathbf{I}
$$

The deformation gradient tensor $\mathbf{F}$ is defined as,

$$
\mathbf{F}=\frac{\partial \mathbf{x}}{\partial \mathbf{X}}
$$

and is a two-point tensor since references to the deformed and the undeformed configurations.

Rearranging (2.8) and using (2.9), the deformation gradient can be expressed as a function of the displacement field.

$$
\mathbf{F}=\frac{\partial \mathbf{U}}{\partial \mathbf{X}}+\mathbf{I}
$$

The determinant of the deformation gradient tensor $\mathbf{F}$ is known as the Jacobian,

$$
J=\operatorname{det}(\mathbf{F})
$$

and represents the change of volume.

Applying the polar decomposition to the deformation gradient $\mathbf{F}$,

$$
\mathbf{F}=\mathbf{R} \cdot \mathbf{U}=\mathbf{v} \cdot \mathbf{R}
$$

where $\mathbf{U}, \mathbf{v}^{1}$ are the right and left stretch tensors, respectively, and $\mathbf{R}$ is the rotation tensor. Because $\mathbf{U}, \mathbf{v}$ are symmetric and positive definite second order tensors, it is possible to diagonalize them by using the coordinate transformation defined by their respective eigenvectors. The values of the diagonal terms are defined by the associated eigenvalues. This procedure is also known as spectral decomposition.

When the left stretch tensor $\mathbf{v}$ is transformed to principal coordinates, takes the form

$$
\mathbf{v}=\left[\begin{array}{ccc}
\lambda_{1} & 0 & 0 \\
0 & \lambda_{2} & 0 \\
0 & 0 & \lambda_{3}
\end{array}\right]
$$

\footnotetext{
${ }^{1}$ Note that the displacement field and the right stretch tensors share the same symbol, $\mathbf{U}$, although they represent different variables. From here on, $\mathbf{U}$ will be used to represent the right stretch tensor.
} 
where $\lambda_{1}, \lambda_{2}$ and $\lambda_{3}$ are the principal stretches, which are calculated as the eigenvalues of $\mathbf{v}$.

It can be proved that

$$
\operatorname{det}(\mathbf{R})=1
$$

Recalling the following property from determinants,

$$
\operatorname{det}(\mathbf{A} \cdot \mathbf{B})=\operatorname{det}(\mathbf{A}) \operatorname{det}(\mathbf{B})
$$

and, substituting (2.12) and (2.14) in (2.11), it can be seen that

$$
J=\operatorname{det}(\mathbf{F})=\operatorname{det}(\mathbf{v} \cdot \mathbf{R})=\operatorname{det}(\mathbf{v}) \operatorname{det}(\mathbf{R})=\operatorname{det}(\mathbf{v})=\lambda_{1} \lambda_{2} \lambda_{3}
$$

The deformation gradient $\mathbf{F}$ can be decomposed into volume-changing (dilatational) and volume-preserving (distortional) parts using the following multiplicative decomposition,

$$
\mathbf{F}=\left(J^{1 / 3} \mathbf{I}\right) \mathbf{F}^{*}=J^{1 / 3} \mathbf{F}^{*}
$$

where $\mathbf{F}^{*}$ is the distortional part and $\left(J^{1 / 3} \mathbf{I}\right)$ is the dilatational part.

It also will be useful to introduce the velocity gradient $\mathbf{L}$,

$$
L_{i, j}=\frac{\partial v_{i}}{\partial x_{j}}
$$

where the velocity $\mathbf{v}$ is given by,

$$
v_{i}=\frac{\partial x_{i}}{\partial t}
$$

Using the chain-rule, it is possible to express (2.18) as

$$
L_{i, j}=\frac{\partial v_{i}}{\partial X_{a}} \frac{\partial X_{a}}{\partial x_{j}}
$$

Replacing $v$ by its definition (2.19), and using Clairaut's theorem,

$$
\frac{\partial v_{i}}{\partial X_{a}}=\frac{\partial}{\partial X_{A}}\left(\frac{\partial x_{i}}{\partial t}\right)=\frac{\partial}{\partial t}\left(\frac{\partial x_{i}}{\partial X_{A}}\right)=\frac{\partial \mathbf{F}}{\partial t}=\dot{\mathbf{F}}
$$

And noting that

$$
\frac{\partial X_{a}}{\partial x_{j}}=\mathbf{F}^{-1},
$$

it is possible to express $\mathbf{L}$ in terms of $\mathbf{F}$ and its time derivative [31, Equation 2.139].

$$
\mathbf{L}=\dot{\mathbf{F}} \cdot \mathbf{F}^{-1}
$$

Any second order tensor can be decomposed into a symmetric and anti-symmetric (skewsymmetric) parts. For the velocity gradient tensor $\mathbf{L}$, this additive decomposition yields 


$$
\mathbf{L}=\mathbf{D}+\mathbf{W}
$$

with

$$
\begin{aligned}
\mathbf{D} & =\frac{1}{2}\left(\mathbf{L}+\mathbf{L}^{T}\right) \\
\mathbf{W} & =\frac{1}{2}\left(\mathbf{L}-\mathbf{L}^{T}\right)
\end{aligned}
$$

where $\mathbf{D}, \mathbf{W}$ are the rate of deformation and spin tensors, respectively.

Furthermore, the total deformation gradient $\mathbf{F}$ can be decomposed using a multiplicative decomposition

$$
\mathbf{F}=\mathbf{F}^{e} \cdot \mathbf{F}^{p}
$$

where $\mathbf{F}^{e}$ is the elastic component and $\mathbf{F}^{p}$ is the viscous or plastic component.

The Left Cauchy-Green deformation tensor (also known as Finger tensor) B is written in terms of the spatial (deformed) coordinates as

$$
\mathbf{B}=\mathbf{F} \cdot \mathbf{F}^{T}
$$

Note that it is named as the left deformation tensor because the deformation gradient $\mathbf{F}$ is written unaltered to the left of the dot in the right hand side of (2.28).

The left Cauchy-Green deformation tensor of the distortional part of the deformation $\mathbf{B}^{*}$ can be written in terms of $\mathbf{F}^{*}$ as

$$
\mathbf{B}^{*}=\mathbf{F}^{*} \cdot \mathbf{F}^{* T}=J^{-2 / 3} \mathbf{F} \cdot \mathbf{F}^{T}
$$

Any tensor can be decomposed into a deviatoric part and a spherical part using an additive decomposition.

$$
\begin{aligned}
\mathbf{A} & =\alpha \mathbf{I}+\operatorname{dev}[\mathbf{A}] \\
\alpha & =\frac{\operatorname{tr}[\mathbf{A}]}{3}
\end{aligned}
$$

The deviatoric part is noted using the deviatoric operator $\operatorname{dev}[]$ and from (2.30) and (2.31),

$$
\operatorname{dev}[]=[]-\frac{\operatorname{tr}[]}{3} \mathbf{I}
$$

\subsection{Stress Measures}

The Cauchy stress tensor $\boldsymbol{\sigma}$ is also know as the true stress tensor because the traction vectors obtained when multiplied by a normal vector, represent the force applied per unit area in the deformed configuration. In the other hand, the first Piola-Kirchhoff stress tensor $\mathbf{P}$ is 


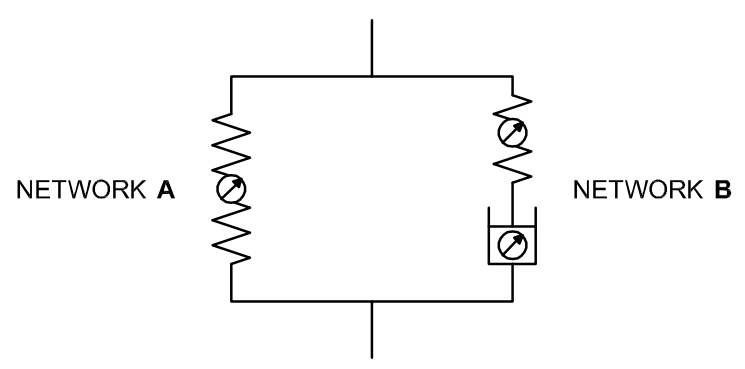

Figure 2.1: BB Model Scheme

known as the nominal stress tensor because considers the undeformed area. The relationship between both can be calculated using Nanson's formula and is given by,

$$
\mathbf{P}=J \boldsymbol{\sigma} \cdot \mathbf{F}^{-T}
$$

\subsection{Bergström - Boyce Material Model}

The Bergström - Boyce Material Model (BB Model) has the ability to reproduce the hyperelastic and rate-dependent viscous behavior of rubber-like polymers. The most simple viscoelastic models, e.g. Prony Series, are lineal models. Thus, they can not capture rate dependency in the viscous behavior. One of the principal objectives of the present work is to calculate the dissipation of the Actuated Polymer Film as a function of the frequency of the excitation. Therefore, a material model with a hysteretic behavior sensitive to the frequency is needed.

The BB model is relatively new and has been implemented in general purpose Finite Element Analysis packages like ABAQUS ${ }^{\circledR}$ and ANSYS ${ }^{\circledR}$ with different success [1].

\subsubsection{Constitutive and Kinematic Model}

The BB model can be conceptually thought as a two networks in parallel. Network A characterizes the response when the viscous effects are not present (equilibrium), while Network $\mathrm{B}$ carries the time dependent component (non-equilibrium). A schematic representation of this model is given in Figure 2.1.

The dials in the springs and dashpot in Figure 2.1 reflect that the response of these elements is not linear. In order to account for finite strains, the spring elements should be modeled with a hyperelastic model. Different models could be used in the networks A and $\mathrm{B}$, but they are usually modeled using the same kind of hyperelastic model.

Because of the parallel nature of the BB model, both branches are subjected to the same deformation gradient $\mathbf{F}$ [13, 17,

$$
\mathbf{F}=\mathbf{F}_{A}=\mathbf{F}_{B}
$$

Given (2.34) is possible to apply the decomposition given by (2.27) to $\mathbf{F}_{B}$ 


$$
\mathbf{F}_{B}=\mathbf{F}_{B}^{e} \cdot \mathbf{F}_{B}^{p}
$$

In a parallel system, all the branches are subjected to the same deformation gradient. But each branch can, in general, have a different stress. Therefore the resulting stress will be sum of the stresses from each branch. For the BB model there are only two networks, thus the resulting stress will be

$$
\boldsymbol{\sigma}=\boldsymbol{\sigma}_{A}+\boldsymbol{\sigma}_{B}
$$

where $\boldsymbol{\sigma}$ is the Cauchy (true) stress tensor.

The next steps will be to find the strain-stress relationships of networks A and B.

\section{Network A}

Here, a relationship between the elastic part of the deformation gradient $\mathbf{F}^{e}$ and the Cauchy stress tensor $\sigma_{A}$ must be defined. For this purpose any hyperelastic model can be used.

In the present work the path followed by Bergstrom and Boyce [13, 11, 15, 16] will be taken, adopting the eight-chain network model proposed by Arruda and Boyce [4]. In this model, the Cauchy stress tensor is given by

$$
\boldsymbol{\sigma}_{A}=\frac{\mu_{A}}{J \overline{\lambda^{*}}} \frac{\mathscr{L}^{-1}\left(\overline{\lambda^{*}} / \lambda_{A}^{\text {lock }}\right)}{\mathscr{L}^{-1}\left(1 / \lambda_{A}^{\text {lock }}\right)} \operatorname{dev}\left[\mathbf{B}^{*}\right]+\kappa(J-1) \mathbf{I}
$$

where $\mu_{A}, \lambda_{A}^{\text {lock }}, \kappa$ are the hyperelastic parameters of network A (initial shear modulus, limiting chain stretch and bulk modulus, respectively) and $\mathbf{B}_{A}^{*}=\mathbf{B}_{B}^{*}=\mathbf{B}^{*} \cdot \mathscr{L}^{-1}(x)$ is the inverse Langevin function and $\overline{\lambda^{*}}$ is the effective distortional chain stretch given by

$$
\overline{\lambda^{*}}=\sqrt{\operatorname{tr}\left[\mathbf{B}^{*}\right] / 3}
$$

The Langevin function is given by

$$
\mathscr{L}(\beta)=\operatorname{coth} \beta-\frac{1}{\beta}
$$

The following approximation to the inverse Langevin function is proposed by Bergstrom [12],

$$
\mathscr{L}^{-1}(x)= \begin{cases}1.31446 \tan (1.58986 x)+0.91209 x, & \text { if }|x|<0.84136 \\ 1 /(\operatorname{sign}(x)-x), & \text { if } 0.84136 \leq|x|<1\end{cases}
$$

In this manuscript, the series expansion approximation (2.41) of the inverse Langevin will be used [45, 37].

$$
\mathscr{L}^{-1}(x) \approx 3 x+\frac{9}{5} x^{3}+\frac{297}{175} x^{5}+\frac{1539}{875} x^{7}+\frac{126117}{67375} x^{9}+\ldots
$$

Although (2.40) has been reported to perform slightly better than (2.41) [12], the latter will be used because is what ABAQUS ${ }^{\circledR}$ uses in its formulation [26, Section 4.6.1]. Therefore, it is sound to use the same expression both in the fitting of the material parameters and in the finite element simulations. 


\section{Network B}

Within network B, the elastic and viscous elements are laid in series. Thus, they share the same stress but the deformation in each element might be different. The stress on the network $\mathrm{B}$ is calculated in a similar fashion as it was done for network A, using the hyperelastic 8chain model by Arruda and Boyce, but in this case only the elastic part of the deformation gradient $\mathbf{F}_{B}^{e}$ should be used,

$$
\boldsymbol{\sigma}_{B}=\frac{\mu_{B}}{J_{B}^{e} \overline{\lambda_{B}^{e *}}} \frac{\mathscr{L}^{-1}\left(\overline{\lambda_{B}^{e *}} / \lambda_{B}^{\text {lock }}\right)}{\mathscr{L}^{-1}\left(1 / \lambda_{B}^{\text {lock }}\right)} \operatorname{dev}\left[\mathbf{B}_{B}^{e *}\right]+\kappa\left[J_{B}^{e}-1\right] \mathbf{I}
$$

where $\mu_{B}$ and $\lambda_{B}^{\text {lock }}$ are the parameters of the hyperelastic part of network B. It is common to express $\mu_{B}$ as a function of $\mu_{A}$, introducing the parameter $s$

$$
\mu_{B}=\mu_{A} s
$$

And usually, it is assumed that the bulk modulus $\kappa$ and the limiting chain stretch $\lambda^{\text {lock }}$ is the same for both networks,

$$
\lambda^{\text {lock }}=\lambda_{A}^{\text {lock }}=\lambda_{B}^{\text {lock }}
$$

The time dependent part of network B is analyzed introducing its velocity gradient $\mathbf{L}_{B}$. Substituting (2.27) in (2.23),

$$
\begin{aligned}
\mathbf{L}_{B} & =\dot{\mathbf{F}}_{B} \cdot \mathbf{F}_{B}^{-1} \\
& =\overline{\left(\mathbf{F}_{B}^{e} \cdot \mathbf{F}_{B}^{p}\right)} \cdot\left(\mathbf{F}_{B}^{e} \cdot \mathbf{F}_{B}^{p}\right)^{-1} \\
& =\left(\mathbf{F}_{B}^{e} \cdot \dot{\mathbf{F}}_{B}^{p}+\dot{\mathbf{F}}_{B}^{e} \cdot \mathbf{F}_{B}^{p}\right) \cdot \mathbf{F}_{B}^{p^{-1}} \cdot \mathbf{F}_{B}^{e^{-1}} \\
& =\mathbf{F}_{B}^{e} \cdot \mathbf{F}_{B}^{p} \cdot \mathbf{F}_{B}^{p^{-1}} \cdot \mathbf{F}_{B}^{e^{-1}}+\mathbf{F}_{B}^{e} \cdot \mathbf{F}_{B}^{p} \cdot \mathbf{F}_{B}^{p^{-1}} \cdot \mathbf{F}_{B}^{e^{-1}} \\
& =\mathbf{F}_{B}^{e} \cdot \mathbf{L}_{B}^{p} \cdot \mathbf{F}_{B}^{e^{-1}}+\mathbf{F}_{B}^{e} \cdot \mathbf{I} \cdot \mathbf{F}_{B}^{e^{-1}} \\
& =\underbrace{\mathbf{F}_{B}^{e} \cdot \mathbf{L}_{B}^{p} \cdot \mathbf{F}_{B}^{e^{-1}}}_{\widetilde{\mathbf{L}_{B}^{p}}}+\mathbf{L}_{B}^{e} \\
& =\widetilde{\mathbf{L}_{B}^{p}}+\mathbf{L}_{B}^{e}
\end{aligned}
$$

Using (2.24) it is possible to decompose the tensor $\widetilde{\mathbf{L}_{B}^{p}}$ into the rate of deformation and spin tensors,

$$
\widetilde{\mathbf{L}_{B}^{p}}=\widetilde{\mathbf{D}_{B}^{p}}+\widetilde{\mathbf{W}_{B}^{p}}
$$

In the derivation of the $\mathrm{BB}$ model it is assumed that the spin tensor is equal to zero, $\widetilde{\mathbf{W}_{B}^{p}}=0$. Under this assumption, it is possible to rewrite $(2.45)$ as

$$
\mathbf{L}_{B}=\widetilde{\mathbf{D}_{B}^{p}}+\mathbf{L}_{B}^{e}
$$

The rate of deformation of the network B is assumed to follow the flow rule given by, 


$$
\widetilde{\mathbf{D}_{B}^{p}}=\dot{\gamma}_{B} \frac{\mathbf{S}_{B}}{\tau_{B}}
$$

where $\dot{\gamma}_{B}$ is the effective viscous strain rate in network $\mathrm{B}, \mathbf{S}_{B}$ is the deviatoric part of the Cauchy stress tensor $\boldsymbol{\sigma}_{B}$ acting in the network B and $\tau_{B}$ is an effective stress in network B defined by the Frobenius norm of $\mathbf{S}_{B}$.

$$
\tau_{B}=\left\|\mathbf{S}_{B}\right\|_{F}=\sqrt{\left.\operatorname{tr} \overline{\mathbf{S}_{B}^{T}} \cdot \mathbf{S}_{B}\right]}
$$

where $\overline{\mathbf{S}_{B}^{T}}$ is the conjugate transpose of $\mathbf{S}_{B}$. Being $\mathbf{S}_{B}$ a real and symmetric second order tensor,

$$
\tau_{B}=\sqrt{\operatorname{tr}\left[\mathbf{S}_{B} \cdot \mathbf{S}_{B}\right]}=\sqrt{\mathbf{S}_{B}: \mathbf{S}_{B}}
$$

The effective viscous strain rate in network B $\dot{\gamma}_{B}$ is driven by

$$
\dot{\gamma}_{B}=\left(\frac{\dot{\gamma}_{0}}{\tau_{\text {base }}^{m}}\right)\left(\overline{\lambda_{B}^{p}}-1+E\right)^{c} \tau_{B}^{m}
$$

where $\dot{\gamma}_{0} / \tau_{\text {base }}^{m}, c$ and $m$ are material parameters, $E$ is a constant added to improve the numerical convergence and $\overline{\lambda_{B}^{p}}$ is the chain stretch given by

$$
\overline{\lambda_{B}^{p}}=\sqrt{\frac{\operatorname{tr}\left[\mathbf{F}_{B}^{p^{T}} \cdot \mathbf{F}_{B}^{p}\right]}{3}}
$$

The left hand side of the flow rule (2.48) can be replaced by $\mathbf{F}_{B}^{e} \cdot \mathbf{F}_{B}^{p} \cdot \mathbf{F}_{B}^{p^{-1}} \cdot \mathbf{F}_{B}^{e^{-1}}$ from (2.45),

$$
\mathbf{F}_{B}^{e} \cdot \dot{\mathbf{F}}_{B}^{p} \cdot \mathbf{F}_{B}^{p^{-1}} \cdot \mathbf{F}_{B}^{e^{-1}}=\dot{\gamma}_{B} \frac{\mathbf{S}_{B}}{\tau_{B}}
$$

Solving for $\mathbf{F}_{B}^{p}$,

$$
\begin{aligned}
\dot{\mathbf{F}}_{B}^{p} & =\frac{\dot{\gamma}_{B}}{\tau_{B}} \mathbf{F}_{B}^{e^{-1}} \cdot \mathbf{S}_{B} \cdot \underbrace{\mathbf{F}_{B}^{e} \cdot \mathbf{F}_{B}^{p}}_{\mathbf{F}_{B}=\mathbf{F}_{A}=\mathbf{F}} \\
& =\frac{\dot{\gamma}_{B}}{\tau_{B}} \mathbf{F}_{B}^{e^{-1}} \cdot \mathbf{S}_{B} \cdot \mathbf{F}
\end{aligned}
$$

\subsection{Uniform Uniaxial Deformation and Incompressible Material}

In this section the uniform uniaxial deformation will be developed. This type of deformation is very important because it corresponds to the case of tensile/compressive testing. Later 


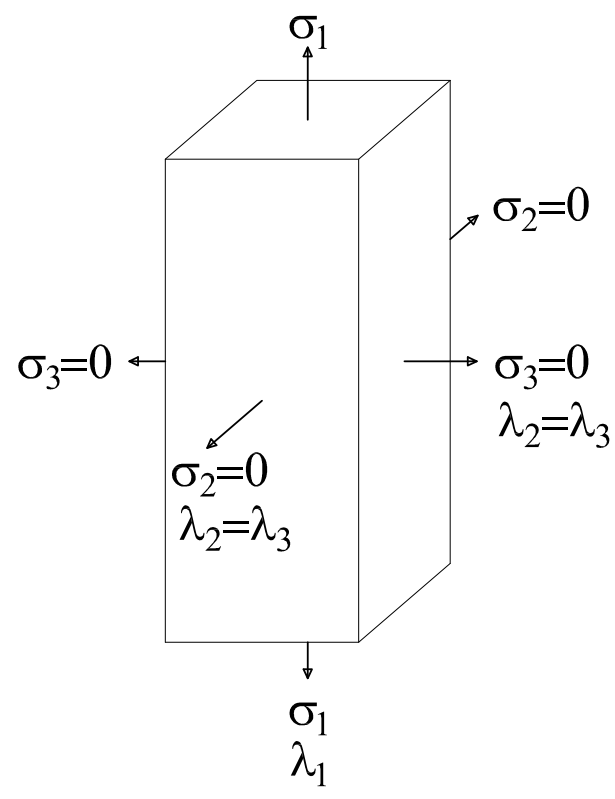

Figure 2.2: Uniaxial Deformation

on, it will be used for fitting material parameters to experimental data. Many rubber-like materials and elastomers show an incompressible or near incompressible behavior, allowing to introduce the incompressibility hypothesis. If the material it is assumed to incompressible, then

$$
J=\operatorname{det}(\mathbf{F})=1
$$

Under this assumption the following relationship must be satisfied,

$$
\lambda_{1} \lambda_{2} \lambda_{3}=1
$$

In uniaxial deformation, two of the stretches are equal between them [30]. Arbitrary picking $\lambda_{2}=\lambda_{3}$ and replacing in (2.56), it it possible to solve for $\lambda_{2}$

$$
\lambda_{2}=\sqrt{\frac{1}{\lambda_{1}}}
$$

Also, in uniaxial deformation there are no rotations. Therefore,

$$
\mathbf{R}=\mathbf{I} \quad \Rightarrow \quad \mathbf{F}=\mathbf{v}
$$

and the deformation gradient $\mathbf{F}$ becomes 


$$
\mathbf{F}=\left[\begin{array}{ccc}
\lambda & 0 & 0 \\
0 & 1 / \sqrt{\lambda} & 0 \\
0 & 0 & 1 / \sqrt{\lambda}
\end{array}\right]
$$

Replacing (2.59) into (2.29),

$$
\mathbf{B}^{*}=\left[\begin{array}{ccc}
\lambda^{2} & 0 & 0 \\
0 & 1 / \lambda & 0 \\
0 & 0 & 1 / \lambda
\end{array}\right]
$$

With the explicit form of $\mathbf{B}^{*}$ for the uniaxial and incompressible case, it is possible to write the equations for the effective distortional chain stretch $\overline{\lambda^{*}}$,

$$
\overline{\lambda^{*}}=\sqrt{\operatorname{tr}\left[\mathbf{B}^{*}\right] / 3}=\sqrt{\frac{1}{3}\left(\lambda^{2}+\frac{2}{\lambda}\right)}
$$

and for the deviatoric part of the distortional deformation $\operatorname{dev}\left[\mathbf{B}^{*}\right]$,

$$
\begin{aligned}
\operatorname{dev}\left[\mathbf{B}^{*}\right] & =\mathbf{B}^{*}-\frac{1}{3} \operatorname{tr}\left[\mathbf{B}^{*}\right] \mathbf{I} \\
\operatorname{dev}\left[\mathbf{B}^{*}\right] & =\left[\begin{array}{ccc}
\lambda^{2} & 0 & 0 \\
0 & 1 / \lambda & 0 \\
0 & 0 & 1 / \lambda
\end{array}\right]-\frac{1}{3}\left(\lambda^{2}+\frac{2}{\lambda}\right)\left[\begin{array}{lll}
1 & 0 & 0 \\
0 & 1 & 0 \\
0 & 0 & 1
\end{array}\right]
\end{aligned}
$$

The state of stress for the uniaxial deformation is given by,

$$
\boldsymbol{\sigma}=\left[\begin{array}{ccc}
\sigma_{11} & 0 & 0 \\
0 & 0 & 0 \\
0 & 0 & 0
\end{array}\right]
$$

The relationship between the true stress and the nominal stress for the uniaxial case can be calculated using (2.33),

$$
\mathbf{P}=J\left[\begin{array}{ccc}
\sigma_{11} & 0 & 0 \\
0 & 0 & 0 \\
0 & 0 & 0
\end{array}\right] \cdot\left[\begin{array}{ccc}
1 / \lambda & 0 & 0 \\
0 & \sqrt{\lambda} & 0 \\
0 & 0 & \sqrt{\lambda}
\end{array}\right]=\left[\begin{array}{ccc}
\sigma_{11} / \lambda & 0 & 0 \\
0 & 0 & 0 \\
0 & 0 & 0
\end{array}\right]
$$

Therefore, the relationship between the nominal and true stress in the loading direction is

$$
P_{11}=\frac{\sigma_{11}}{\lambda}
$$

as seen in (2.6). 


\subsubsection{Application to the BB Model}

Replacing (2.62) in (2.37) it is possible to express the Cauchy stress tensor of the network A as a function of the stretch in the direction of the uniaxial strain $\lambda$,

$$
\begin{array}{r}
\boldsymbol{\sigma}_{A}=\frac{\mu_{A}}{J \overline{\lambda^{*}}} \frac{\mathscr{L}^{-1}\left(\overline{\lambda^{*}} / \lambda_{A}^{\text {lock }}\right)}{\mathscr{L}^{-1}\left(1 / \lambda_{A}^{\text {lock }}\right)}\left\{\left[\begin{array}{ccc}
\lambda^{2} & 0 & 0 \\
0 & 1 / \lambda & 0 \\
0 & 0 & 1 / \lambda
\end{array}\right]-\frac{1}{3}\left(\lambda^{2}+\frac{2}{\lambda}\right)\left[\begin{array}{ccc}
1 & 0 & 0 \\
0 & 1 & 0 \\
0 & 0 & 1
\end{array}\right]\right\}+ \\
\kappa(J-1)\left[\begin{array}{lll}
1 & 0 & 0 \\
0 & 1 & 0 \\
0 & 0 & 1
\end{array}\right]
\end{array}
$$

Although the factor $(J-1)$ in the last term of $(2.66)$ is equal to zero because incompressibility was assumed $(J=1)$, it will be useful to keep it.

Under the uniaxial deformation assumption and for an isotropic material, the only nonzero term of the stress tensor is $\sigma_{A_{11}}$. Writing explicitly the stress component $\sigma_{A_{22}}$,

$$
\sigma_{A_{22}}=\frac{\mu_{A}}{J \overline{\lambda^{*}}} \frac{\mathscr{L}^{-1}\left(\overline{\lambda^{*}} / \lambda_{A}^{\text {lock }}\right)}{\mathscr{L}^{-1}\left(1 / \lambda_{A}^{\text {lock }}\right)}\left\{\frac{1}{3}\left(\frac{1}{\lambda}-\lambda^{2}\right)\right\}+\kappa(J-1) 1
$$

and equating it to zero $\sigma_{A_{22}}=0$, it is possible to solve for $\kappa(J-1)$,

$$
\kappa(J-1)=\frac{\mu_{A}}{J \overline{\lambda^{*}}} \frac{\mathscr{L}^{-1}\left(\overline{\lambda^{*}} / \lambda_{A}^{\text {lock }}\right)}{\mathscr{L}^{-1}\left(1 / \lambda_{A}^{\text {lock }}\right)}\left\{\frac{1}{3}\left(\lambda^{2}-\frac{1}{\lambda}\right)\right\}
$$

The result obtained would be the same if instead of choosing $\sigma_{A_{22}}, \sigma_{A_{33}}$ was used.

Lastly, replacing (2.68) in (2.66) the only non-zero stress component $\sigma_{A_{11}}$ is expressed in terms of the stretch in the loading direction [35].

$$
\sigma_{A_{11}}=\frac{\mu_{A}}{\overline{\lambda^{*}}} \frac{\mathscr{L}^{-1}\left(\overline{\lambda^{*}} / \lambda_{A}^{\text {lock }}\right)}{\mathscr{L}^{-1}\left(1 / \lambda_{A}^{\text {lock }}\right)}\left(\lambda^{2}-\frac{1}{\lambda}\right)
$$

Similarly, for the stress component $\sigma_{B_{11}}$ in network B,

$$
\sigma_{B_{11}}=\frac{\mu_{B}}{\overline{\lambda_{B}^{e *}}} \frac{\mathscr{L}^{-1}\left(\overline{\lambda_{B}^{e *}} / \lambda_{B}^{\text {lock }}\right)}{\mathscr{L}^{-1}\left(1 / \lambda_{B}^{\text {lock }}\right)}\left(\lambda_{B}^{e^{2}}-\frac{1}{\lambda_{B}^{e}}\right)
$$

where the effective distortional chain stretch in the elastic part of network B is given by

$$
\overline{\lambda_{B}^{e *}}=\sqrt{\frac{1}{3}\left(\left(\lambda_{B}^{e *}\right)^{2}+\frac{2}{\lambda_{B}^{e *}}\right)}
$$

For the uniaxial state,

$$
\mathbf{S}_{B}=\operatorname{dev}\left[\boldsymbol{\sigma}_{B}\right]=\frac{\sigma_{B_{11}}}{3}\left[\begin{array}{ccc}
2 & 0 & 0 \\
0 & -1 & 0 \\
0 & 0 & -1
\end{array}\right]
$$




$$
\begin{gathered}
\tau_{B}=\sqrt{\operatorname{tr}\left[\mathbf{S}_{B} \cdot \mathbf{S}_{B}\right]}=\left|\sigma_{B_{11}}\right| \sqrt{\left(\frac{2}{3}\right)^{2}+2\left(\frac{-1}{3}\right)^{2}}=\sqrt{\frac{2}{3}}\left|\sigma_{B_{11}}\right| \\
\mathbf{F}_{B}^{e^{-1}}=\left[\begin{array}{ccc}
1 / \lambda_{B}^{e} & 0 & 0 \\
0 & \sqrt{\lambda_{B}^{e}} & 0 \\
0 & 0 & \sqrt{\lambda_{B}^{e}}
\end{array}\right]
\end{gathered}
$$

Substituting (2.72), (2.73) and (2.74) in the flow rule (2.54),

$$
\begin{aligned}
& \dot{\mathbf{F}}_{B}^{p}=\frac{\dot{\gamma}_{B}}{\sqrt{\frac{2}{3}}\left|\sigma_{B_{11}}\right|}\left[\begin{array}{ccc}
1 / \lambda_{B}^{e} & 0 & 0 \\
0 & \sqrt{\lambda_{B}^{e}} & 0 \\
0 & 0 & \sqrt{\lambda_{B}^{e}}
\end{array}\right] \cdot\left(\frac{\sigma_{B_{11}}}{3}\left[\begin{array}{ccc}
2 & 0 & 0 \\
0 & -1 & 0 \\
0 & 0 & -1
\end{array}\right]\right) . \\
& {\left[\begin{array}{ccc}
\lambda & 0 & 0 \\
0 & 1 / \sqrt{\lambda} & 0 \\
0 & 0 & 1 / \sqrt{\lambda}
\end{array}\right]}
\end{aligned}
$$

Writing the component in the loading direction $\dot{\mathbf{F}}_{B 11}^{p}$, and replacing $\dot{\gamma}_{B}$ by (2.51),

$$
\dot{\lambda}_{B}^{p}=\left(\frac{\dot{\gamma}_{0}}{\tau_{\text {base }}^{m}}\right)\left(\sqrt{\frac{1}{3}\left(\left(\lambda_{B}^{p}\right)^{2}+\frac{2}{\lambda_{B}^{p}}\right)}-1+E\right)^{c}\left(\sqrt{\frac{2}{3}}\left|\sigma_{B_{11}}\right|\right)^{m}\left[\sqrt{\frac{2}{3}} \operatorname{sign}\left(\sigma_{B_{11}}\right)\right] \lambda_{B}^{p}
$$

The above equation is a first-order ordinary differential equation (ODE) in $\lambda_{B}^{p}$. Therefore, only one initial condition is needed to solve the ODE. 


\section{Chapter 3}

\section{BB Model Parameters Fit}

The parameters of the BB Model can be fit to uniaxial experimental data using a MATLAB ${ }^{\circledR}$ program written for this purpose. The program is used to fit available uniaxial experimental data from Reference [10] for Nitrile Rubber (NR), Acrylate-Butadiene Rubber (ABR), Silicone Rubber (SR) and Viton.

The theory behind the BB Model and the particular case of uniaxial deformation have been developed in Chapter 2 .

\subsection{Program Structure}

This program is divided in three files, fit $10 . \mathrm{m}, \mathrm{bb} 10 . \mathrm{m}$ and bb10_error.m. In addition to these files, the non built-in minimization function FMINSEARCHCON.m [27] is used to fit the material model to the experimental data and it should be in the same directory or in the path.

The variables Y_data, X_data, DATA_SETS, ROW_INDEX and LENGTH_DATA_SETS are defined as global and they will be used to pass information between the three parts of the program.

The code listing for this program is included in the Appendix A. The code is thoroughly commented.

\subsubsection{Main Program}

\section{Experimental Data}

The main file of the program is fit $10 . \mathrm{m}$. In this MATLAB ${ }^{\circledR}$ file the experimental data is loaded. The program allows to input the results of more that one experiment. Each set of data is stored in a cell inside the variable M. The variables DATA_SETS, ROW_INDEX and LENGTH_DATA_SETS are used to keep track of how many experiments will be used and how many data points each experiment has.

The experimental data needed to do the uniaxial fit for the BB model is composed of time, strain and stress. The stresses and strains that are input to this program must be true values, not nominal values. If the experimental data is available in the latter fashion, it should be converted to true values using (2.5) and (2.6) . 
The true stress $\sigma$ will be considered to be a function of time $t$ and the true strain $\epsilon$ history. Therefore, $\sigma=\sigma(t, \epsilon(t))$.

The BB model has the ability to capture the rate dependent behavior, characteristic of many elastomers. But in order to do a successful fit of the parameters that define this model, it is imperative that the experimental data is sampled, at least, for two different strain rates.

\section{Initial Values and Bounds}

The initial values for the parameters must be defined in this section. These initial values will be used as a starting point by the solver, and they are stored in the array beta0. The function FMINSEARCHCON allows the use of bound constraints for the parameters to fit. This feature is used in this problem because the value of some of the parameters of the BB model are restricted to certain ranges. For example, the initial shear modulus of network $\mathrm{A}, \mu_{A}$ must be positive. Also the creep strain exponent $c$ must be in the $[-1,0]$ interval. The lower and upper bounds for the parameters are stored in the LB and UB arrays, respectively. For unbounded values, the bound is set as $\infty$ using the inf command in MATLAB ${ }^{\circledR}$.

\section{Set the minimization algorithm options}

The options for the termination of FMINSEARCHCON must be specified here.

TolFun Tolerance on the function to minimize. If the difference between two consecutive iterations of the value of the function to minimize is less that TolFun the iteration process will be terminated.

TolX Tolerance on the parameters. Similar to TolFun, but the difference is evaluated on the value of the parameters that are being fitted.

MaxFunEvals Maximum number of evaluations of the function to minimize.

MaxIter Maximum number of iterations to be used during the minimization.

Usually the set of points to be fit is very large. Therefore, the computational time required to do this kind of non-linear minimization is considerable. It was found that the most effective parameter to control the time spent on the optimization is MaxIter. The improvement on the goodness of fit parameter $R^{2}$, changes marginally when MaxIter is changed from 10 to 100 . The results that will be presented later in this Chapter have been calculated using MaxIter $=10$.

\section{Call the minimization algorithm}

Once the initial values and bounds of the parameters and the options for the minimization algorithm have been set, the program actually calls to FMINSEARCHCON.

A function that quantifies the difference between the experimental values and the predicted values must be defined. The error function is the function that the will be minimized by FMINSEARCHCON. This function will be described in Section 3.1.3, 


\section{Output}

Once the minimization algorithm has finished, either because it has converged to the defined criteria or because it has exceeded the maximum number of iterations, the main program will show the estimated parameters and it will calculate the goodness of fit $R^{2}$.

\subsubsection{Constitutive Equation Function}

In this Section the constitutive equation for the uniaxial loading case of the Bergstrom-Boyce material model will be numerically implemented. Given a strain-time curve, the constitutive equation will calculate the stress. In order to do this, the ordinary differential equation (2.76) must be solved. In this program the solution of the ODE is done using MATLAB ${ }^{\circledR}$ 's built-in ode23 solver.

At the beginning of each experimental data set, the strain and stress are known and equal to zero. Therefore, the total stretch is equal to the elastic and viscous stretches, $\lambda=\lambda_{B}^{e}=\lambda_{B}^{p}=1$. This will be the initial condition for the ODE solver for the first time step of every experimental data set. The ODE solver will integrate the flow rule (2.76) from the current time step to the following. Once it solves for viscous stretch $\lambda_{B}^{p}$ for the following time step, the elastic stretch $\lambda_{B}^{e}$ is calculated using the multiplicative decomposition given by (2.35), particularized for the uniaxial case $\left(\lambda=\lambda_{B}^{e} \lambda_{B}^{p}\right)$.

Having the elastic stretch for network B $\lambda_{B}^{e}$ and the total stretch $\lambda$, it is possible to find the stress in both networks employing (2.69) and (2.70).

At this point, all the ingredients required to start the next iteration are available. The viscous stretch $\lambda_{B}^{p}$ calculated at this step will become the initial condition to solve the ODE for the following step.

\subsubsection{Error Function}

The error function uses the constitutive equation function developed in the previous section and calculates the difference between the stress calculated by the constitutive equation and the experimental stress. The smaller the difference, the better the fit is. There are different functions that can be used to measure the error. In the present work the Normalized Median Absolute Difference will be used.

$$
\operatorname{ERROR}=100 \frac{\sum_{i=1}^{n}\left|\sigma_{i}^{\exp }-\sigma_{i}^{\mathrm{fit}}\right|}{\sum_{i=1}^{n}\left|\sigma_{i}^{\exp }\right|}
$$

where $n$ is the number of experimental data points, $\sigma^{\exp }$ is vector containing all the measured stresses and $\sigma^{\mathrm{fit}}$ is the vector containing the stresses calculated using the constitutive law and the current value of the parameters. The minimization process is driven by reducing the error given by (3.1). 


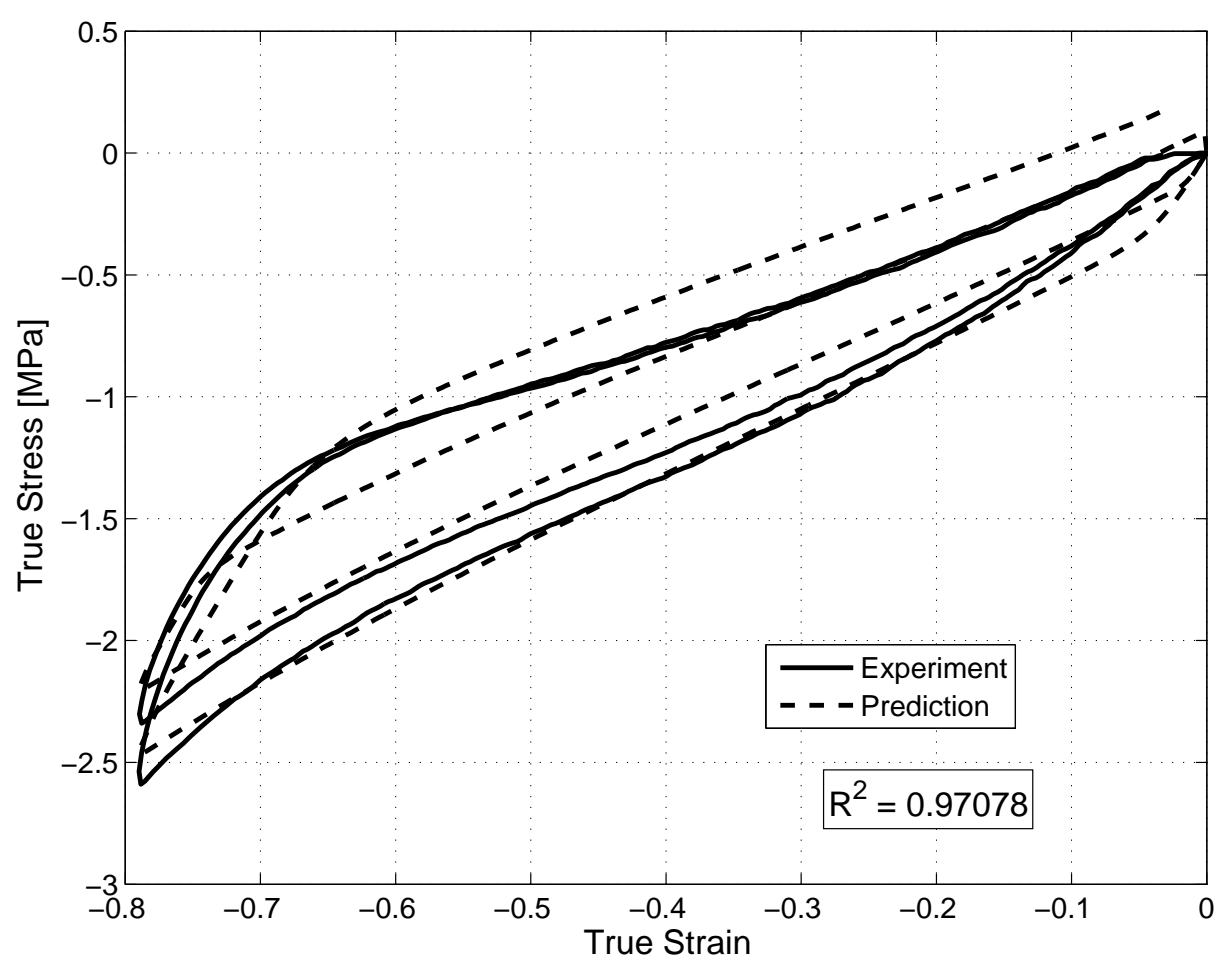

Figure 3.1: Acrylate-Butadiene Rubber (ABR)

\section{$3.2 \quad$ Examples}

In this Section the parameters for the BB model are calculated for four different materials. The experimental data was obtained from the Dr. Bergstrom's website [10]. The calculated parameters and the coefficient of determination $R^{2}$ are included in Table 3.1.

For the four cases the maximum number of iterations (MaxIter) was set to 10. For each material two or three experimental data sets were available, except for Viton. For this material only two data sets were available, but one of them could not be used because some inconsistencies in the data. For the other three cases, the coefficients of determination are higher than 0.95 .

The stress-strain plots of the experimental data and the predicted values are included in Figures 3.1 - 3.4. 


\begin{tabular}{|c|c|cccccc|c|c|}
\hline Material & $R^{2}$ & $\mu_{A}$ & $\lambda_{\text {lock }}$ & $s$ & $\dot{\gamma}_{0} / \tau_{\text {base }}^{m}$ & $c$ & $m$ & MaxIter & Data Sets \\
\hline ABR & 0.971 & 0.910 & 2.073 & 2.251 & 0.686 & -0.471 & 2.406 & 10 & Compression1 and 2 \\
NR & 0.976 & 1.130 & 6.382 & 2.300 & 0.540 & -0.990 & 4.500 & 10 & Nitrile01,02 and 03 \\
Viton & 0.898 & 1.633 & 1.282 & 1.736 & 0.398 & -0.909 & 9.852 & 10 & Viton_slow $^{1}$ \\
SR & 0.952 & 0.830 & 5.224 & 2.636 & 0.527 & -0.624 & 4.393 & 10 & Silicone01, 02 and 03 \\
\hline
\end{tabular}

Table 3.1: Parameters fit for the BB material model

\footnotetext{
${ }^{a}$ The second data set available, Viton_fast.txt could not be used in the fit because, for some unknown reason, there were several duplicated times with different values of stress and strain.
} 


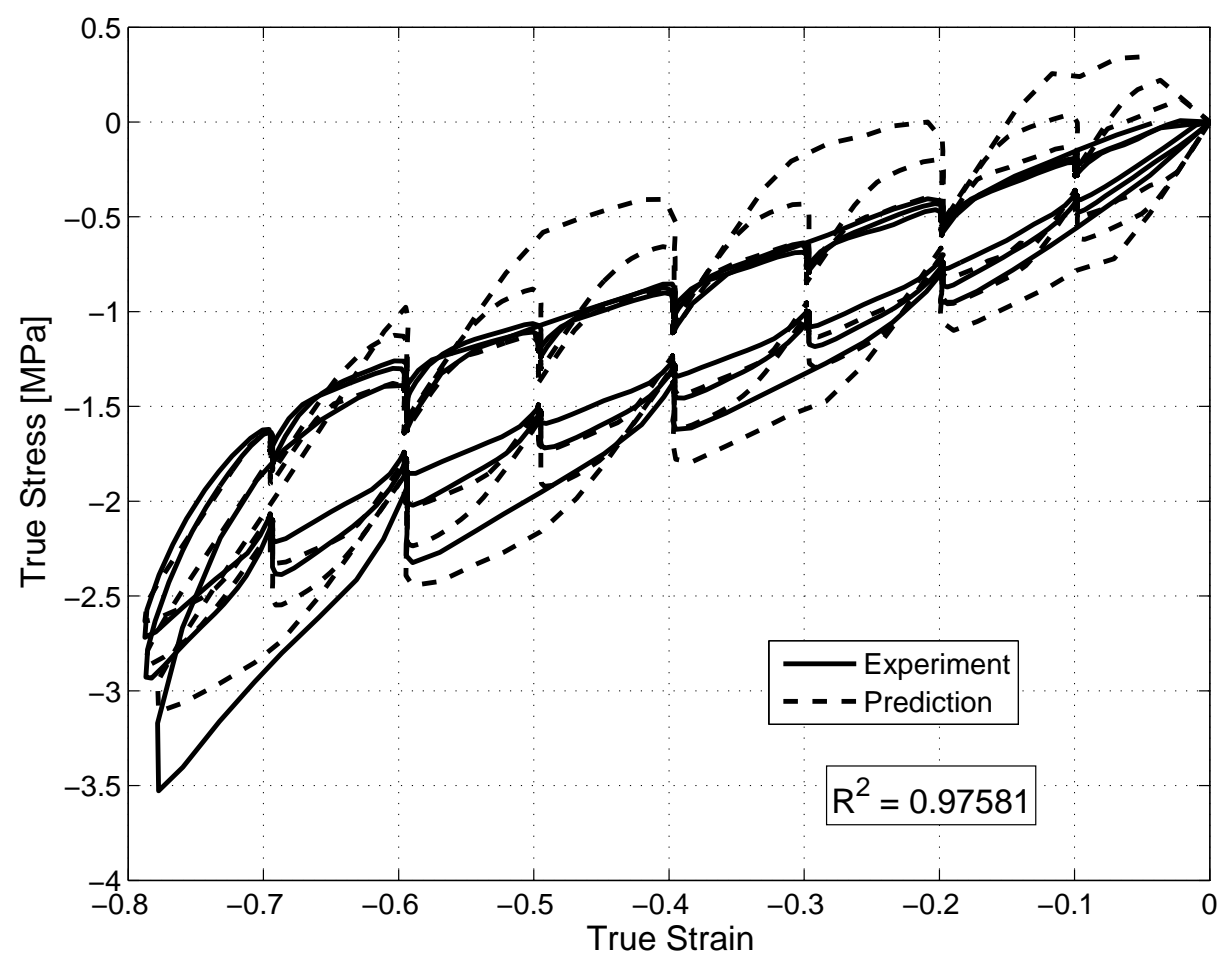

Figure 3.2: Nitrile Rubber

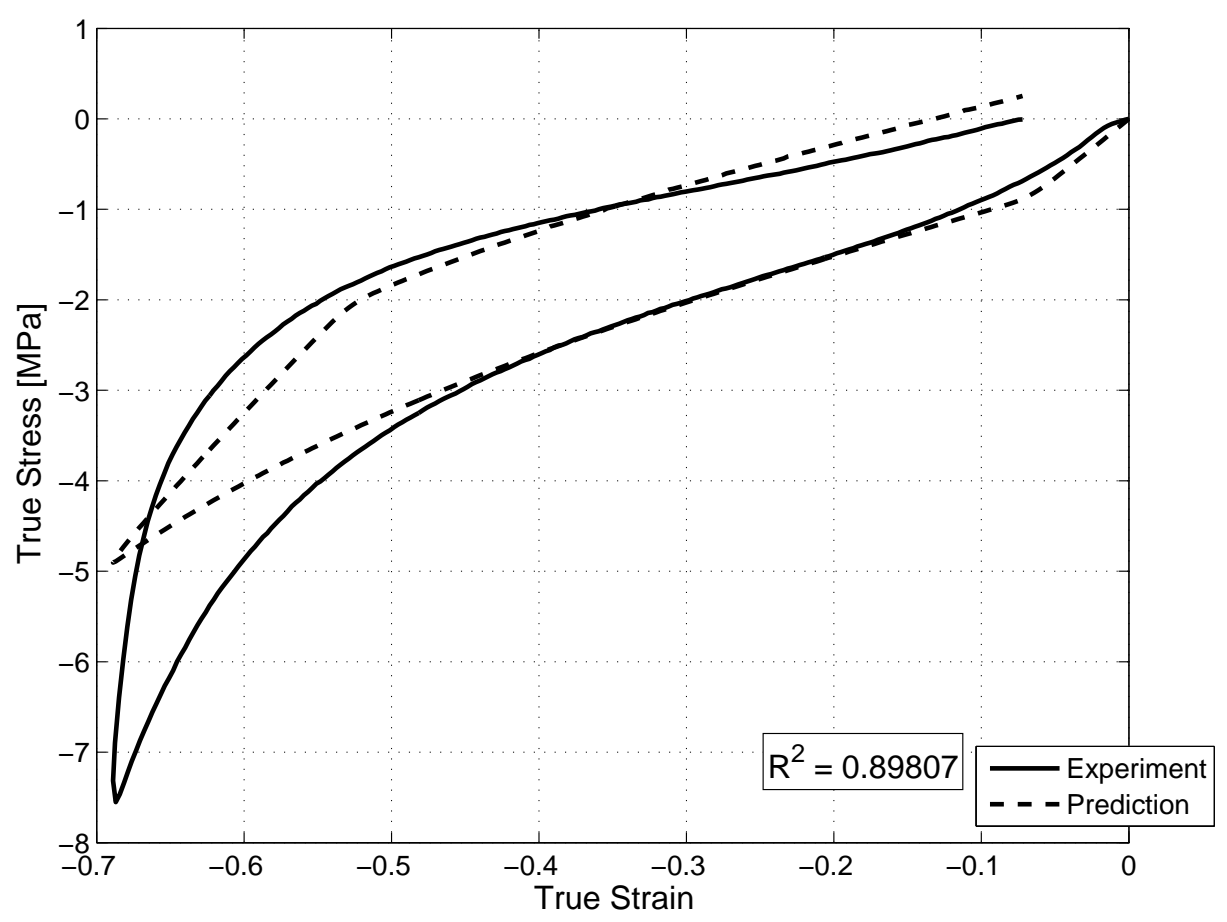

Figure 3.3: Viton 


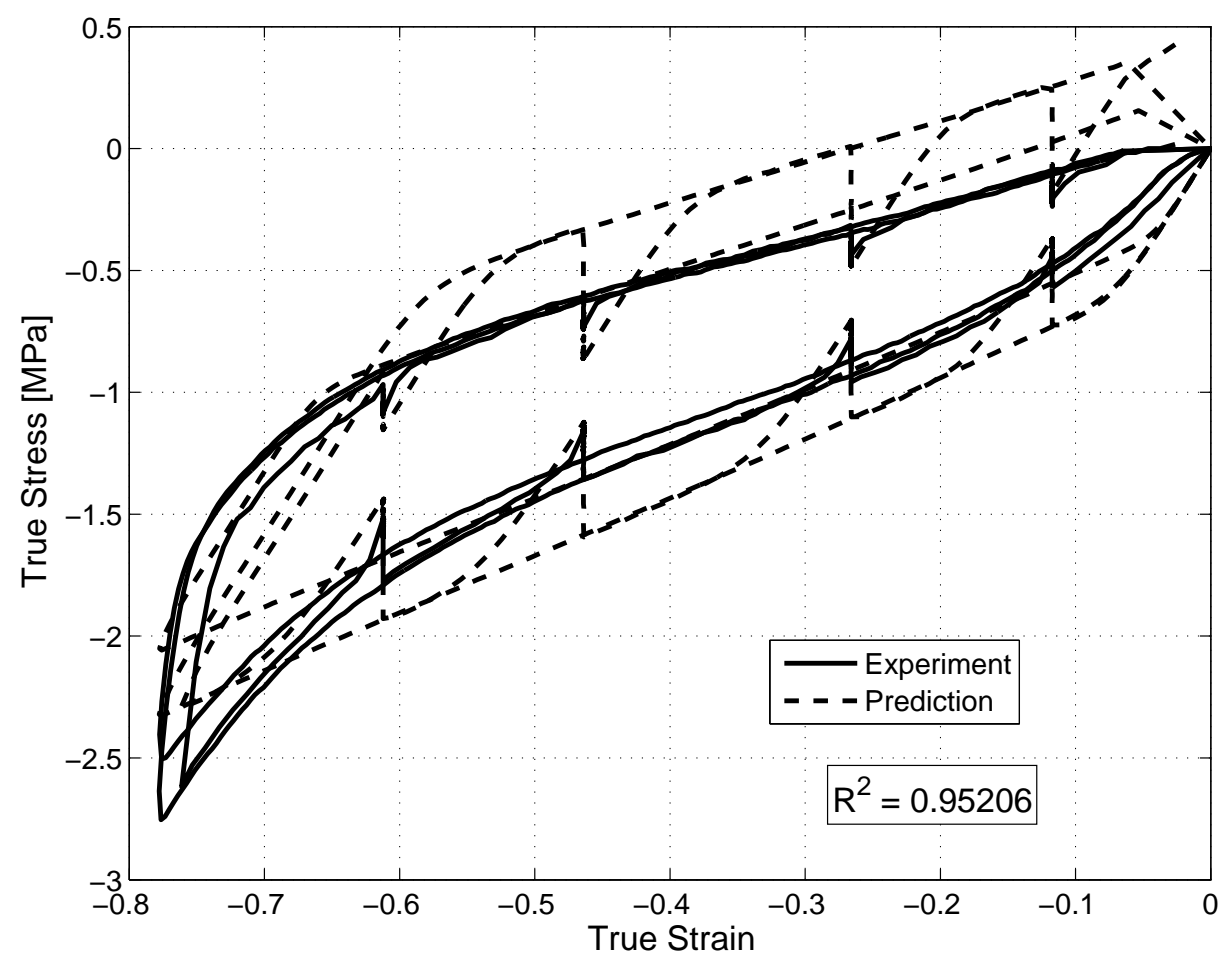

Figure 3.4: Silicone Rubber 


\section{Chapter 4}

\section{Finite Element Analysis}

\subsection{Introduction}

In this Chapter four different types of models will be analyzed. In this Section the parameters that are common to all the models will be introduced.

\subsubsection{Units}

The base units used in the models included in this Chapter are newtons $(\mathrm{N})$ and millimeters $(\mathrm{mm})$. Thus, the stresses and modulus are measured in mega-pascals (MPa).

\subsubsection{Geometry}

The dimensions of each model will be described in the respective sections. It will be considered that the width of the RVE is along the $x$ direction, the thickness is along the $y$ direction and that the depth runs along the $z$ direction. The $x, y, z$ coordinate system and a schematic drawing of the RVE are included in Figure 4.10.

\subsubsection{Material Models and Properties}

In the development of the finite element models, three materials are defined. One for the actuator (ellipse in two-dimensional models and ellipsoid in three-dimensional models), other for the base film where the actuator is embedded and lastly, one for the top film. The top film must be electrically conductive, in order to apply the voltage potential to actuate the ellipses/ellipsoids! 1 .

\section{Actuator}

The actuator is modeled using a linear elastic material model, with Young's modulus $E=$ $3000 \mathrm{MPa}$ and Poisson ratio $\nu=0.3$. The Young's modulus used is based on an upper value

\footnotetext{
${ }^{1}$ The words ellipsoid and ellipse will used interchangeably, although strictly speaking ellipsoid should be used only for three-dimensional geometries while ellipse for two-dimensional geometries.
} 
estimation [20]. The effect of changing the actuator Young's modulus will be studied in Section 4.3.6.

\section{Base Film}

The base film is modeled using the Bergstrom-Boyce Model (BB Model) [13, 14]. The equilibrium (relaxed) response follows the hyperlastic model proposed by Arruda and Boyce [4] with material parameters fit to DuPont's Hytrel 4056 (TPE) elastomer. The equilibrium (relaxed) properties were obtained from CAMPUS Database [19] and are listed in Table 4.1.

The base film material will be assumed to be incompressible, a reasonable hypothesis for most elastomers. Under the assumption of incompressibility many simplifications can be done in the kinematic equations but some special considerations must be taken when applying the finite element method.

It is important to bear in mind that this material was chosen as a starting point in order to build the models. A thorough analysis should be made to asses which material has the optimum properties (mechanical, optical, processing, etc.) for a particular application. Additionally, it should be noted that the analyses have been done at a single arbitrary temperature $\left(23^{\circ} \mathrm{C}\right)$. Shall the intended application will be used at a different temperature (or at variable temperatures), this should be taken into account in the model. This can be done using the time-temperature superposition [8].

The hyperlastic model can be changed if other model fits better the available data. For example, some trials have been done using the Polynomial Strain Energy Potential (degree 2). But as Hytrel 4056 is being used just as an example and it is not the material that will be used in the application, there is no point of choosing a particular model at this stage.

The parameters of the Arruda-Boyce hyperelastic model that fit the data in Table 4.1 were calculated using ABAQUS ${ }^{\circledR}$ and are $\mu_{A}=4.68 \mathrm{MPa}, \lambda_{A}^{\text {lock }}=5311.53$ and $d=0.00 / \mathrm{MPa}$, where $\mu_{A}$ is the shear modulus of network $\mathrm{A}, \lambda_{A}^{\text {lock }}$ is the limiting chain stretch and $d$ is the compressibility parameter.

There are four remaining parameters of the BB Model that characterize the time dependent and hysteretic behavior of the material. Because the data required to fit these parameters is not available from the literature, these parameters were taken from the literature as usual values for polymers. The adopted values are $s=2.0, \dot{\gamma}_{0} / \tau_{\text {base }}^{m}=0.5 /\left(s \mathrm{MPa}^{m}\right), m=4.5$ and $c=-1.0$, where $s$ is the stress scaling factor (2.43), $\dot{\gamma}_{0} / \tau_{\text {base }}^{m}$ is the creep parameter (2.51), $m$ is the effective stress exponent (2.51) and $c$ is the creep strain exponent (2.51).

The procedure developed in Chapter 3 can be used to fit all the required parameters of the $\mathrm{BB}$ model from experimental data for any desired material.

\section{Top Film}

The top film is modeled using a linear elastic material model, with Young's modulus $E=$ $70000 \mathrm{MPa}$ and Poisson ratio $\nu=0.3$. The Young's modulus used is based on an upper value estimation [20]. The effect of changing the top film modulus will be addressed in Section 4.3.6. 


\begin{tabular}{cc|ccc}
\hline$\epsilon^{\text {eng }}[]$ & $\sigma^{\text {eng }}[\mathrm{MPa}]$ & $\epsilon^{\text {eng }}[]$ & $\sigma^{\text {eng }}[\mathrm{MPa}]$ \\
\hline 0.047 & 2.83 & 1.050 & 8.69 \\
0.083 & 4.29 & 1.080 & 8.73 \\
0.127 & 5.50 & 1.130 & 8.79 \\
0.163 & 6.20 & 1.160 & 8.84 \\
0.207 & 6.83 & 1.210 & 8.91 \\
0.243 & 7.21 & 1.240 & 8.96 \\
0.286 & 7.55 & 1.290 & 9.03 \\
0.323 & 7.74 & 1.320 & 9.09 \\
0.366 & 7.92 & 1.370 & 9.17 \\
0.402 & 8.02 & 1.400 & 9.22 \\
0.446 & 8.09 & & 1.450 & 9.30 \\
0.482 & 8.15 & 1.480 & 9.37 \\
0.525 & 8.18 & 1.530 & 9.45 \\
0.562 & 8.20 & 1.560 & 9.52 \\
0.605 & 8.23 & 1.610 & 9.60 \\
0.641 & 8.25 & 1.640 & 9.68 \\
0.685 & 8.29 & 1.680 & 9.78 \\
0.721 & 8.32 & 1.720 & 9.86 \\
0.765 & 8.36 & 1.760 & 10.00 \\
0.801 & 8.39 & 1.800 & 10.10 \\
0.844 & 8.44 & 1.840 & 10.20 \\
0.881 & 8.48 & 1.880 & 10.20 \\
0.924 & 8.53 & & 1.920 & 10.40 \\
0.960 & 8.57 & 1.960 & 10.50 \\
1.000 & 8.62 & & & \\
& & & & \\
& & & \\
0.000 & 10.60
\end{tabular}

Table 4.1: Stress-Strain Curve for Hytrel 4056 (TPE) at $23^{\circ} \mathrm{C}$ [19] 


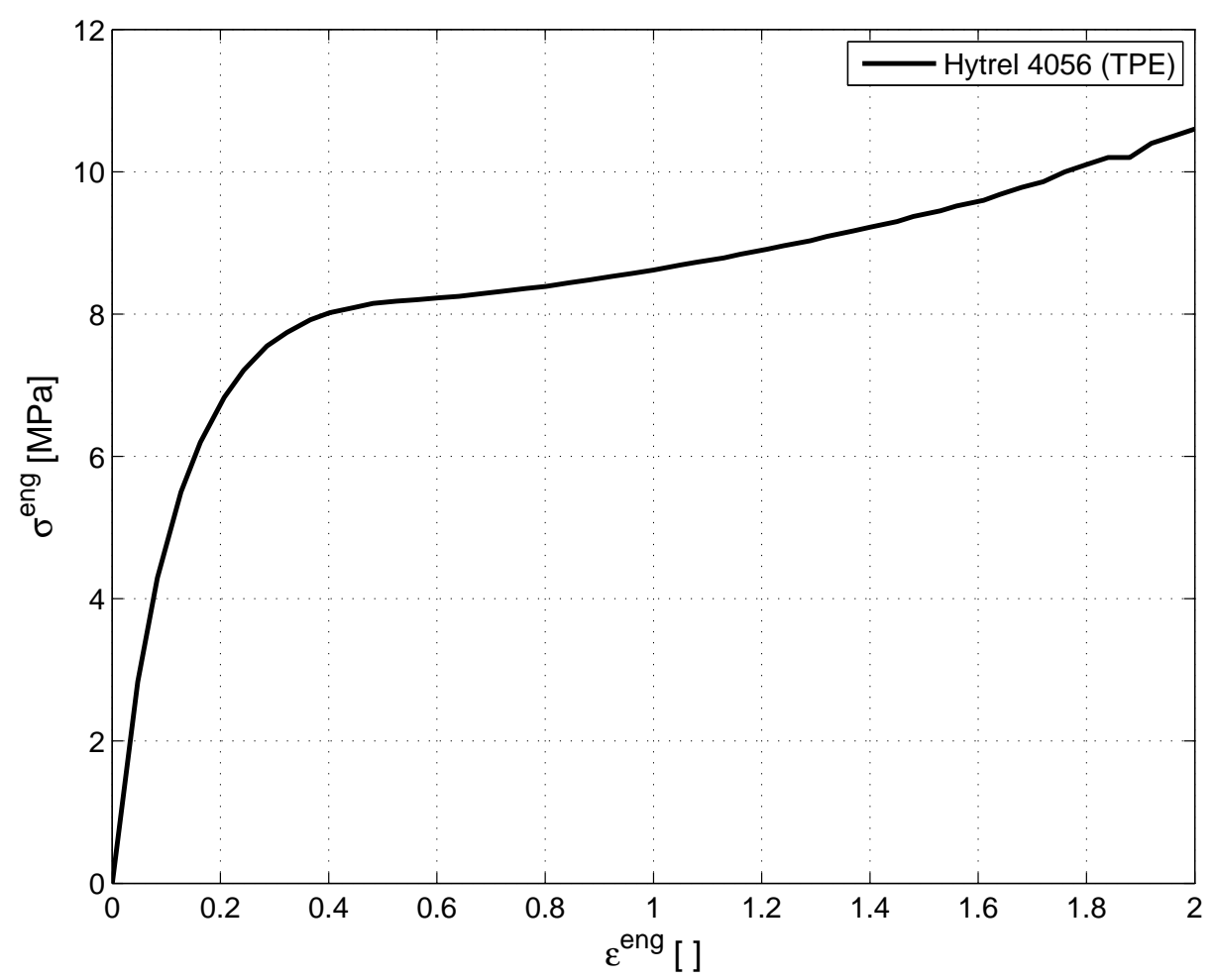

Figure 4.1: Stress-Strain Curves for Hytrel 4056 (TPE) at $23^{\circ} \mathrm{C}$

\subsubsection{Hybrid Elements}

When a material is incompressible or near incompressible, the traditional displacement based finite element formulations (where all the unknowns of the problems are kinematic variables) fail because of a numerical singularity (volumetric locking). The cause of this problem is that very small changes in the displacements (associated with dilatational deformation) cause very large changes in the hydrostatic pressure. The solution is to treat the pressure as an independent unknown. The basis for this approach is given by the mixed or hybrid finite element formulations. In the Hellinger-Reissner mixed method, the displacements and stresses are treated as unknowns. The more general method by $\mathrm{Hu}$-Washizu, also treats the strains as basic variables, in addition to the displacements and stresses [47, 18].

\subsubsection{Periodic Boundary Conditions}

All the models developed in this Chapter, represent a portion of the whole structure. It is assumed that this portion or substructure repeats itself periodically. If the geometry, material properties, boundary conditions and load are periodic, the solution has to be periodic too. When modeling the substructure it is necessary to recover this periodic behavior on the boundaries of the model. This is achieved using periodic boundary conditions [7, $\S 2.3 .4$ and $\S 6.2]$. 


\subsubsection{Geometric Non-Linearity}

In the models analyzed in this Chapter the deformations are finite. Therefore, the current or deformed configurations are no longer similar to the reference configuration, as in the infinitesimal deformation theory [49, §5]. This distinction makes the problem non-linear and the superposition principle is no longer applicable.

\subsubsection{Post-Processing}

\section{Dissipated Energy}

Although ABAQUS ${ }^{\circledR}$ has the option to calculate the creep dissipated energy in the model (History Output ALLCD), the results are not accurate. Therefore, a PYTHON script is used to do this calculation. A simple example showing this flaw is included in Appendix B, For this analyses, the dissipated energy at one point is defined as the difference between the recoverable elastic energy and the integral between the stress-strain curve. Inertial forces, and therefore kinetic energy, are neglected. For the whole model, the problem reduces to do this integration at each integration point and then to integrate over the volume of model. If the resulting dissipation energy is divided by the duration of the analysis, then a dissipation power can be calculated. Lastly, for application purposes, it will be useful to divide the dissipation power by the width and depth of the RVE, which will define the surface dissipation power.

\section{Roughness}

The roughness will be defined as the difference between the maximum and minimum vertical displacement of the top surface of the RVE. For time dependent loadings, a difference can be calculated for each time frame in the solution process. In this case, the roughness will be the maximum of these differences over all the time frames.

\subsection{Models to Compare with the Laboratory Experi- ments}

At this time, December 2010, the research group that has developed the actuated polymer films is researching how to apply the electrically conductive thin coating (top film) to the base film. In the meantime, the electric potential is being applied by placing the film sample between opposing pieces of indium-tin oxide (ITO) glass, materializing the electrodes for the application of a electric potential [42].

\subsubsection{Geometry}

The models will be two-dimensional. Plane strain will be assumed. The RVE will include two ellipses and the top film will not be included. Table 4.2 has the basic dimensions and Figure 4.2 shows a schematic representation. 


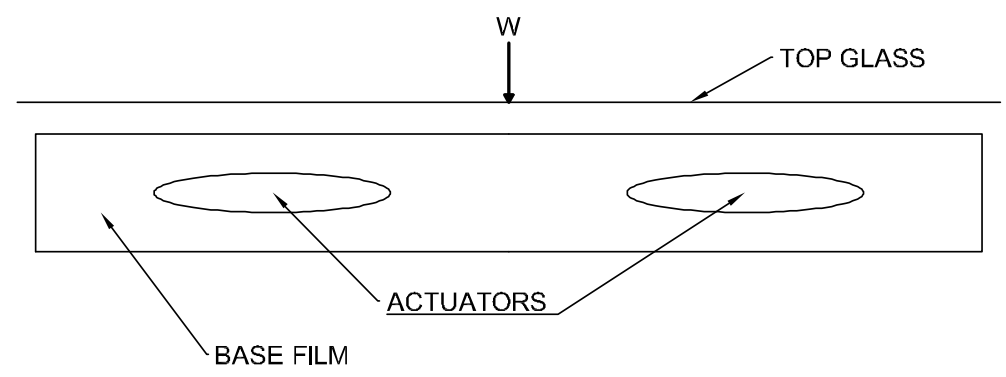

Figure 4.2: Two-Dimensional, Two-Actuators RVE Geometry

\begin{tabular}{l|ccc} 
Component & Width $[\mu \mathrm{m}]$ & Thickness $[\mu \mathrm{m}]$ & Depth $[\mu \mathrm{m}]$ \\
\hline Ellipse & 60 & 10 & $\infty$ \\
Base Film & 240 & 30 & $\infty$
\end{tabular}

Table 4.2: Two Actuators RVE Two-DimensionalGeometry

\subsubsection{Material Properties}

The material properties of the actuator and base film are the ones in Section 4.1.3. For these analyses the viscous behavior is not included. Therefore the base film is modeled using only the hyperlasticity model, without hysteresis.

\subsubsection{Load}

These analyses will be considered as static, this implies than the potential differential should be applied and kept for the duration of the experiment, giving enough time to relax the viscous part of the stress.

The top and bottom surfaces of each ellipse are loaded, respectively, in opposite directions with a uniform distributed load applied in the $x$ direction (parallel to the ellipse major axis) to generate the torque. The magnitude of the the load does not change if the surfaces change their size during the deformation. It could be argued that the magnitude of the load should change when the actuator rotates with respect to the electric field, but the rotations are so small that $\cos \alpha=0.9998$, where $\alpha$ is the maximum rotation angle. The angle was calculated from the model "3D-2" listed in Table 4.4. Also, it is assumed that the direction does not change when the the ellipses deforms (no following forces).

The magnitude of the distributed traction that generates the torque is assumed to be $10 \mathrm{MPa}$. This value was adopted because the resultant displacements from the models are in good agreement (same order of magnitude) with experimental results done in an alike actuated polymer film [42]. Furthermore, it is assumed that the actuators generate torque by a volume and not by an area effect. Cairns et al. propose an equation where the generated torque due to the applied electric field is proportional to the volume $V$ of the actuator, both for cylindrical [21, Equation 1] and spherical [22, Equation 1] geometries. In the latter reference, the torque per unit volume $\Gamma$ is explicitly defined. 
The relationship between the loading for the two- and three-dimensional cases is discussed in Appendix C] In two models the polarity of both ellipses will be the same, while opposite in two other models.

For the finite element models where the top glass is included, a single load (surface traction) magnitude will be analyzed. The effect of changing the magnitude of the surface traction will be studied in Section 4.3.6, where in addition to $10 \mathrm{MPa}$, loads of $5 \mathrm{MPa}$ and $15 \mathrm{MPa}$ will be analyzed.

\subsubsection{Contact}

In these models the effect of the glass resting on the top surface will be included. The top glass will be idealized as a infinitely rigid surface. The kinematics of any rigid body in a two-dimensional space can be described with the three degrees of freedom of any point. This reference point on the analytic rigid surface (top glass) is set at its geometric center. Its mass will be taken as $1.25 \mathrm{~g} / \mathrm{cm}^{2}$, excerpting a pressure of $1.23 \times 10^{-4} \mathrm{MPa}$. The resulting force is applied at the reference point.

A surface to surface, finite sliding algorithm was used in these models. The model was also analyzed using small sliding and both results are in good agreement. The interface between the APF and the glass is assumed to be frictionless. In the surface to surface formulation, some small penetration may occur, but large penetrations of nodes from the master surface into the slave surface are unlikely to occur.

When analyzing contact problems it is important to pay special attention to the following points,

Master-Slave Surfaces The master surface must always be the one that is made of a stiffer material. If both materials have similar stiffness, the surface which has a coarser mesh should be defined as the master surface to minimize the penetration into the slave surface. Following the first criteria, in these examples the analytical rigid surface must be the master.

Normals The normals within each surface should be consistent. The normals of the master surface must point towards the slave surface, and vice versa.

Contact Initiation If the contact between two parts is generated by a force and not by a displacement, it is advisable to create a load step before applying the force where the contact will be generated by a prescribed displacement.

Solver Type When using the finite sliding contact algorithm it is strongly recommended to use a general un-symmetric solver.

\subsubsection{Mesh}

The mesh is composed of 15713 linear, quadrilateral, continuum plane strain elements (See Figure 4.3). The base film is meshed with reduced integration, hybrid elements (CP4RH). Hybrid elements must be used when simulating incompressible materials. ABAQUS ${ }^{\circledR}$ Theory Manual [26, §3.2.4] also suggests that reduced integration elements should be used when 
modeling incompressible materials at finite strain. The actuator is meshed using reduced integration (CP4R) elements, because it is an cost-effective method if the solution is expected to be smooth or if the stresses and/or strains in the region are not of concern.

\subsubsection{Partially Constrained Top Glass Model}

In this Section two different models will be analyzed, none of them having the conductive top film. The purpose of these models is to develop the numerical tools required to, eventually, do a comparison with the experimental results. Each model will include two actuators in the RVE. The difference between the two models is the polarization of both actuators.

Here, the term polarization is used to describe in which direction the actuator will rotate when trying to align with the electric field. The polarization will be considered to be positive when the actuator rotates counter clock-wise from the observer and negative when it does it in a clock-wise fashion. In a sample of APF the polarization of each actuator is random. Therefore is important to analyze the response of the RVE that includes two actuators with the same or opposite polarity.

The PYTHON script used to create and analyze this model in ABAQUS ${ }^{\circledR}$ is listed in the Appendix E.1.

\section{Boundary Conditions}

Base Film The base film is modeled as glued to the bottom glass (not included in the model). Therefore both translational degrees of freedom are set to zero on the bottom surface of the base film. Periodic boundary conditions are applied to the vertical edges of the base film.

Top Glass It is assumed that a glass would be lifted evenly by the combined effect of all the actuators in a sample relatively large. Therefore the rotation of the glass is set to zero. Also the displacement in the horizontal direction is set to zero, leaving only the vertical displacement as an active degree of freedom.

\section{Results}

As it can be seen in Figure 4.4, the difference between the maximum and minimum displacements at the top surface, both for the same and opposite polarity cases, is about the same. For the case in which both actuators have the same polarity, the roughness is $1.89 \mu \mathrm{m}$ and for the the case in which the polarity of each actuator is different, $1.85 \mu \mathrm{m}$. Thus, it could be said that the roughness in both cases is very similar. But in the opposite polarity case, the valley in the center of the RVE is noticeably narrower and more shallow.

Figures 4.5 and 4.6 show the contour plots of the vertical displacement for the same and opposite polarities, respectively. In these plots it can also be seen the analytic rigid surface that models the top glass, in light orange color.

If in the application of the APF, the actuators are close as in these examples and the polarity of each ellipse is random, the overall response of the film would be the average of 


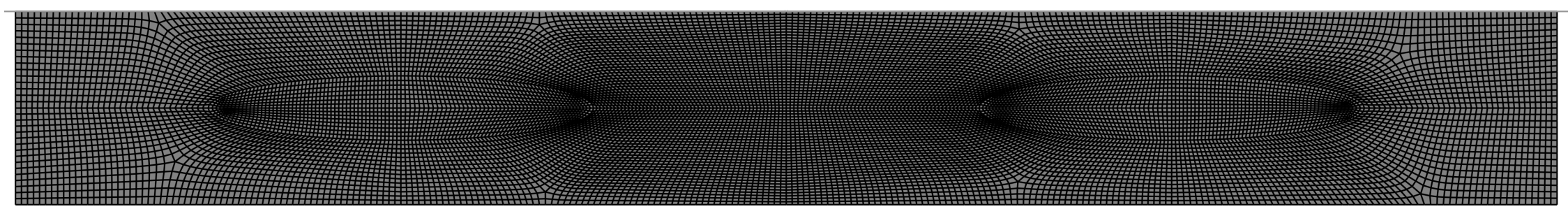

Figure 4.3: Model 4.2 Mesh 


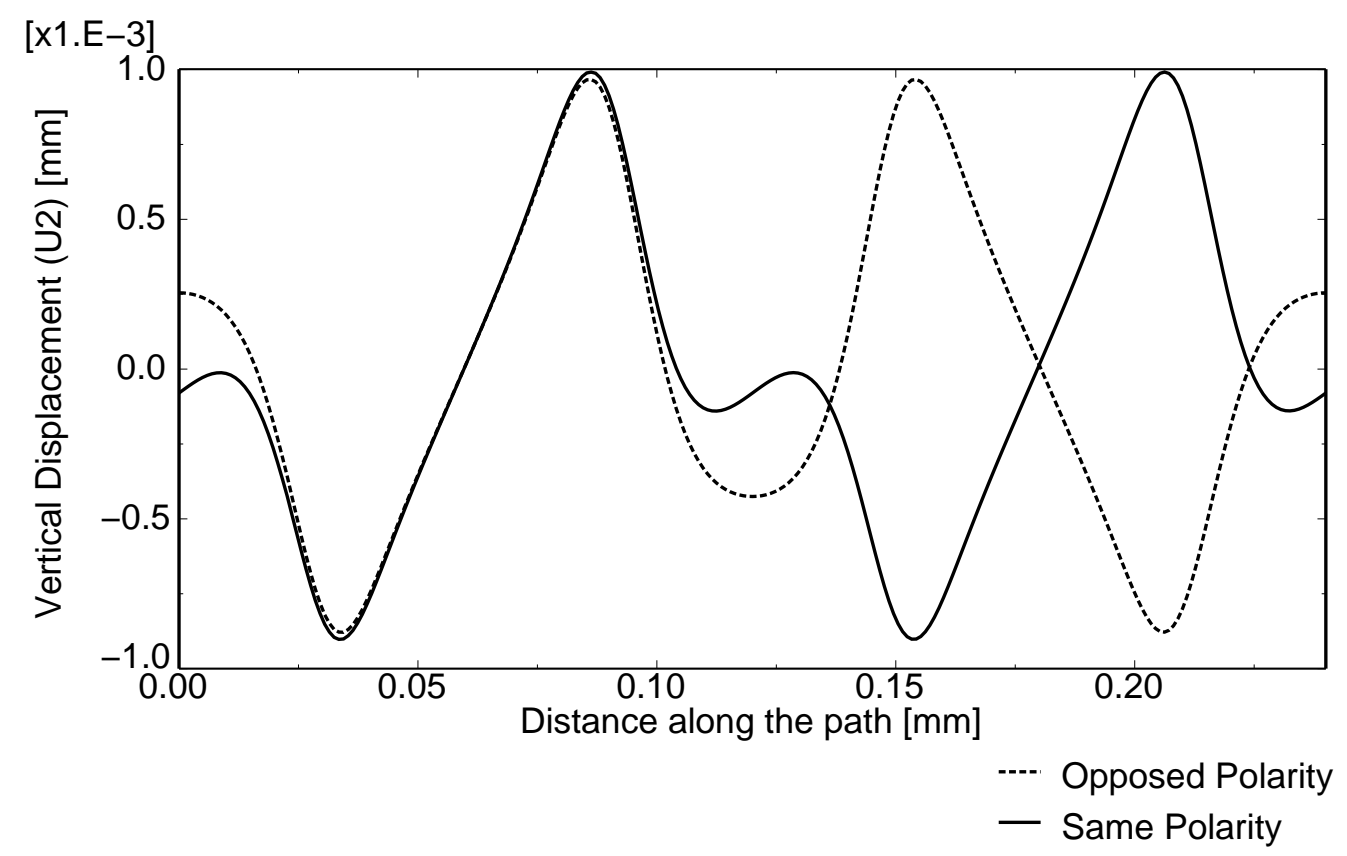

Figure 4.4: Vertical displacement of the top surface of the base film

the two cases treated in this section. If the actuators are far from each other, there will be no neighboring effect and the problem should be modeled as diluted? 2 .

\subsubsection{Totally Constrained Top Glass Model}

The objective by fixing the top glass is to quantify the vertical reaction that the actuated polymer film generates against the top glass.

\section{Boundary Conditions}

Base Film The boundary conditions on the base film are the same as in the previous model, where both translational degrees of freedom are set to zero on the bottom surface of the base film. Also, periodic boundary conditions are applied to the vertical edges of the base film.

Top Glass In these models, the top glass is assumed to have its three degrees of freedom forced to be null. Here, the vertical displacement is assumed to be kept in place by means of a testing device set to displacement control.

\section{Results}

For these models, where the top glass is fully constrained, the reaction force of the rigid analytic surface is very similar regardless the polarization of the two actuators. Therefore,

\footnotetext{
${ }^{2}$ In a diluted model, the separation between the actuators is large enough to consider that the interaction with other actuators is negligible.
} 

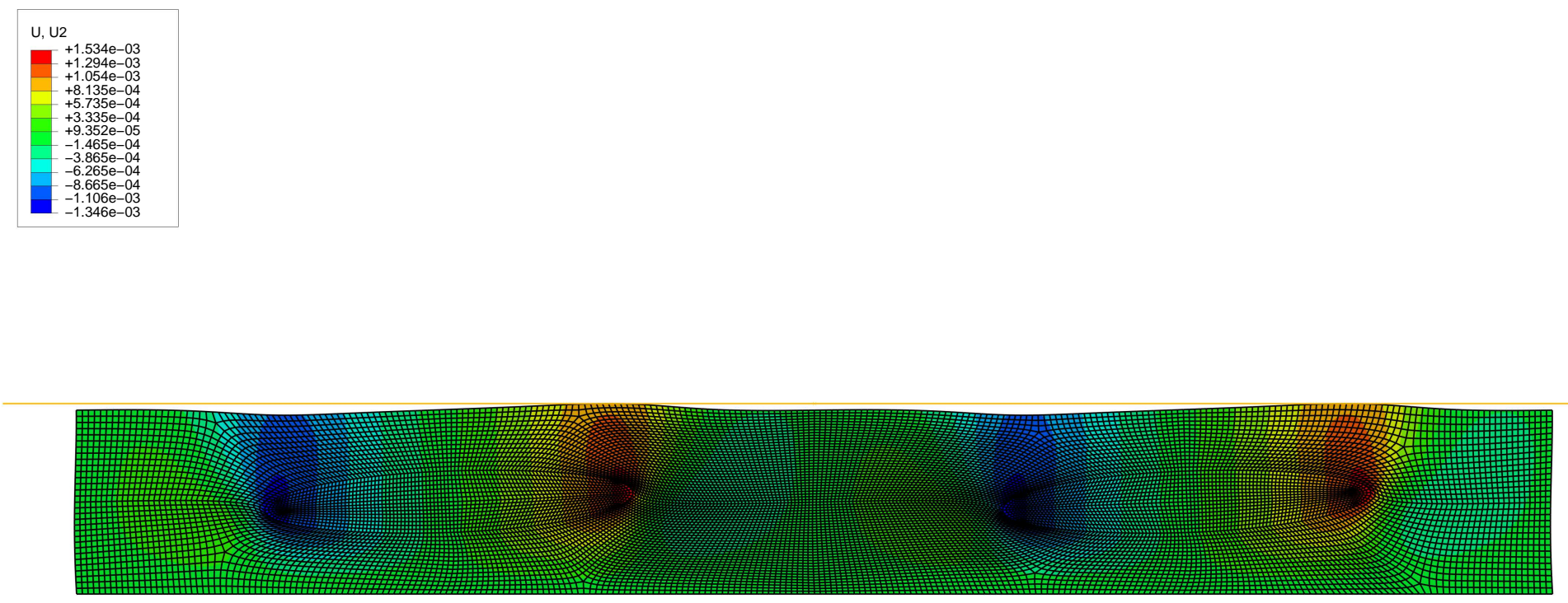

Figure 4.5: Vertical displacement contour plot, actuators with same polarity

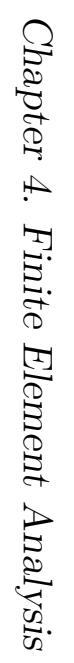



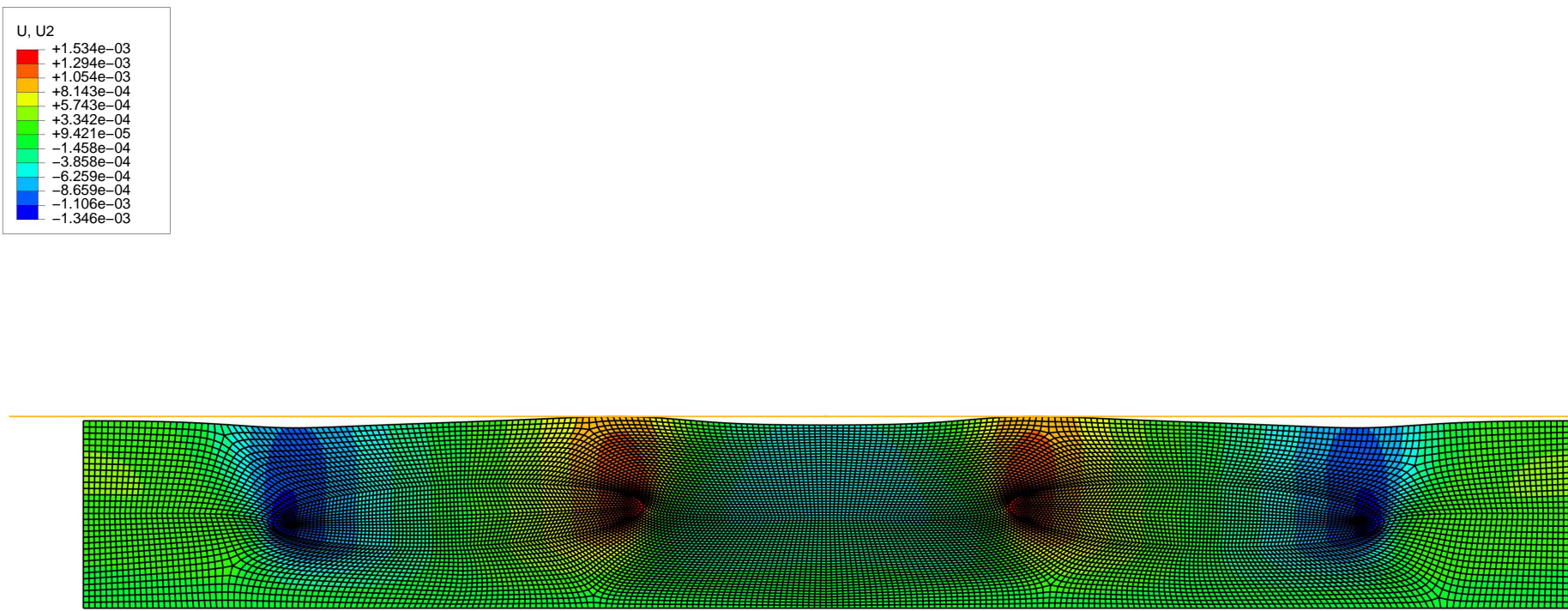

Figure 4.6: Vertical displacement contour plot, actuators with opposite polarity

2
0
0
0
0
0
0
0
0
0
0
0
0
0
0
0
0
0
0
0
0
0
0
0
0 


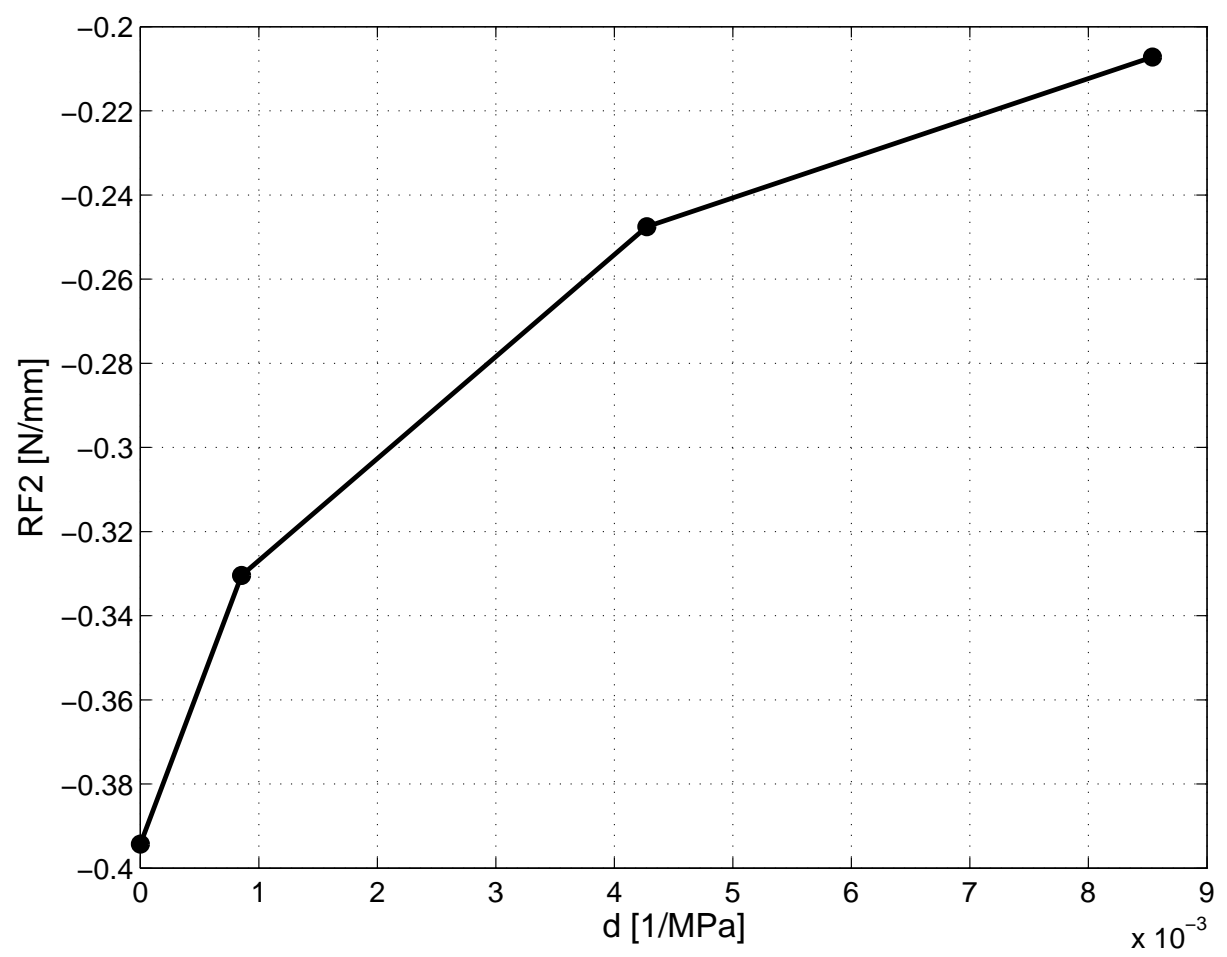

Figure 4.7: Vertical reaction of the top glass as a function of the base film compressibility

only the results for same polarization case will be discussed.

The restriction on the displacement of the top glass, in addition to the glued boundary condition on the bottom of the film and the periodic boundary conditions on the sides of the RVE, highly confines the APF in the model. Under this condition the numerical solution of the problem is highly dependent on the bulk modulus of the material $\kappa$ [16, Appendix A]. The bulk modulus of the material $\kappa$ is related to the compressibility parameter $d$ by

$$
d=\frac{2}{\kappa}
$$

In Figure 4.7, the vertical reaction force at the top glass reference point is plotted against the compressibility parameter $d$. It can be seen that the reaction force is highly dependent on the compressibility parameter. Therefore, the bulk modulus of the base film should be measured accurately in order to compare the reaction force obtained numerically with the experimental measurement.

\subsection{Models of the Application}

In this Section, models of the intended application are analyzed. For some possible applications of the actuated polymer films, like tunable roughness coatings, the electric field is supposed to be applied through an electrically conductive thin film acting as a electrode on the top surface and the substrate where the APF is glued to. Therefore, the top film must be included in the models that will analyze the response of the APF for applications like 
aerodynamic flow control or dust mitigation systems.

It is of special concern to develop the framework that will allow to analyze the response of the actuated polymer films and to calculate the required power to actuate the films. The latter requires to include a hysteretic material model.

In Sections 4.3.3 and 4.3.4 diluted two- and three-dimensional models are developed, respectively. These models have only one actuator embedded in the base film, which is modeled with a width of five times the actuator's length. This is purposely done to analyze the case where the actuators are far from each other (diluted case). The results from these models will be used later, in Section 4.3.5, to correlate the roughness and dissipation between the two- and three-dimensional models, as a function of the volume fraction $V_{f}$.

Lastly, in Section 4.3.6, a series of parametric analyses will be done using a two-dimensional model. The top surface response (roughness) and dissipation power will be analyzed varying the geometry and frequency of excitation. It will be assumed that the three-dimensional behavior can be extrapolated from the two-dimensional results by employing the outcome of Section 4.3.5.

\subsubsection{Material Properties}

The material properties of the actuator, top film and base film are the ones in Section 4.1.3,

\subsubsection{Load}

For the application models the load definition is similar to Section 4.2.3, but in this case the uniform distributed load has a sinusoidal function of time,

$$
F_{x}(t)=A \sin (B t)
$$

where $A$ is the amplitude and $2 \pi B$ is the period of the excitation.

The magnitude $A$ of the surface traction is assumed to be $10 \mathrm{MPa}$, for all but a few cases. In Section 4.3.6, magnitudes of $5 \mathrm{MPa}$ and $15 \mathrm{MPa}$ will be also considered.

The solver is set to a time increment size equal or smaller than $1 / 120$ of the excitation period. The duration of the analysis matches the excitation period. Therefore, at least 120 steps are used to solve the problem. Such a fine time discretization is done because the results from each time step will be used to integrate the dissipation of the model during the post-processing.

\subsubsection{Two-Dimensional Diluted Model}

\section{Geometry}

This model is two-dimensional. Plane strain is assumed. The RVE includes one actuator and the top film. Table 4.3 has the basic dimensions and Figure 4.8 shows a schematic representation of the RVE. 


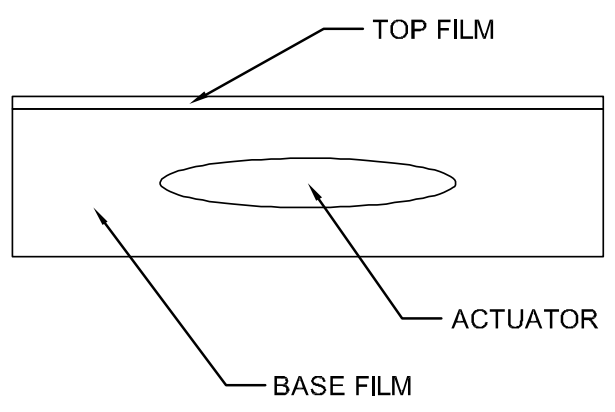

Figure 4.8: Two-Dimensional, One-Actuator Application RVE Geometry

\begin{tabular}{l|ccc} 
Component & Width $[\mu \mathrm{m}]$ & Thickness $[\mu \mathrm{m}]$ & Depth $[\mu \mathrm{m}]$ \\
\hline Ellipse & 60 & 10 & $\infty$ \\
Base Film & 300 & 30 & $\infty$ \\
Top Film & 300 & 0.03 & $\infty$
\end{tabular}

Table 4.3: Two-Dimensional, One Actuator Application RVE Geometry

\section{Load}

The frequency of the load applied by the actuator is $200 \mathrm{~Hz}$. For some of the applications of the APF, the required excitation frequency is expected to be in the $30 \mathrm{~Hz}$ to $200 \mathrm{~Hz}$ range [32. Furthermore, the required frequency is inversely proportional to the roughness. Because the calculated roughness from the finite element analysis results is smaller than the optimum, the top of the frequency range is chosen. In Section 4.3.6, a parametric analysis varying the magnitude and frequency of the excitation will be done. The results are shown in Figures 4.15, 4.16, 4.17 and 4.18.

\section{Boundary Conditions}

The base film is modeled as glued to the bottom glass (not included in the model). Therefore, both translational degrees of freedom are set to zero on the bottom surface of the base film. Periodic boundary conditions are applied to the vertical edges of the RVE.

\section{Mesh}

The elements used are the same type as in the model developed in Section 4.2. For this analysis, only a few (314 out of 13621) constant strain triangular elements are used to improve mesh transitions. The triangular elements used are CPE3 and CPE3H, the former in the actuator and the latter in the base film.

\section{Results}

The dissipation power per unit surface and roughness for this analysis are included in Table 4.5, 


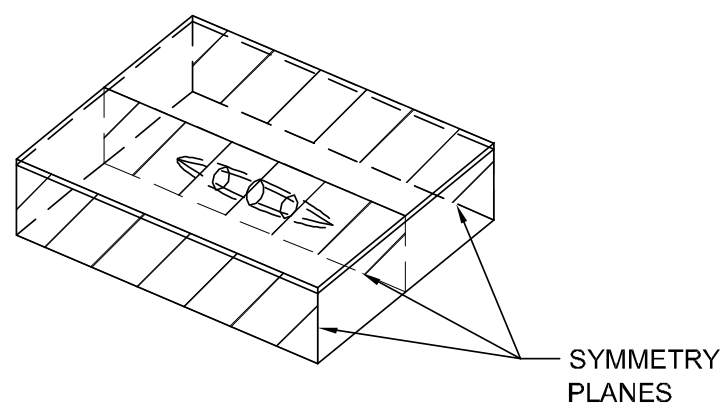

Figure 4.9: Symmetry on the RVE

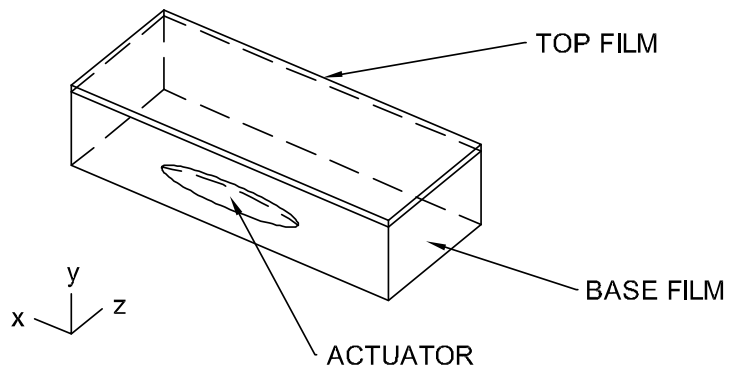

Figure 4.10: Three-Dimensional, One-Actuator Application RVE Geometry

\subsubsection{Three-Dimensional Diluted Model}

These models are three-dimensional. The RVE includes one actuator and the top film. As it can be seen in Figure 4.9, the RVE presents symmetry planes. Symmetry is exploited, so only one half of the RVE is modeled. Table 4.4 has the basic dimensions and Figure 4.10 shows a schematic representation of the RVE.

The PYTHON script used to analyze these models is listed in the Appendix E.3.

\section{Geometry}

Four different volume fractions $(0.70 \%, 1.39 \%, 1.92 \%$ and $3.32 \%)$ will be analyzed, by changing the depth of the RVE $(5.25 \mu \mathrm{m}, 9.81 \mu \mathrm{m}, 12.50 \mu \mathrm{m}$ and $25.00 \mu \mathrm{m})$. With the current process used to create the actuated polymer films there is a minimum theoretical separation

\begin{tabular}{l|ccc} 
Component & Width $[\mu \mathrm{m}]$ & Thickness $[\mu \mathrm{m}]$ & Depth $[\mu \mathrm{m}]$ \\
\hline Ellipsoid & 60 & 10 & 5 \\
Base Film & 300 & 30 & Variable: $5.25-25$ \\
Top Film & 300 & 0.03 & Variable: $5.25-25$
\end{tabular}

Table 4.4: Three-Dimensional, One Actuator Application RVE Geometry 
between actuators in the direction of the depth of the RVE (See Appendix (D), given by

$$
\min \mathrm{DEPTH}=a \sqrt[3]{\mathrm{AR}}
$$

where $a$ is the minor radii of the actuator and AR is the aspect ratio (4.5). For these examples, $a=5 \mu \mathrm{m}$ and $\mathrm{AR}=6$. Therefore, the minimum theoretical depth is $9.1 \mu \mathrm{m}$. Three of the four cases span between the minimum depth and the equivalent to a separation of 5 minor diameters between the ellipsoids centers. The fourth case $($ DEPTH $=5.25 \mu \mathrm{m})$ is included to have a solution close to what it would be the plane strain condition, used in Section 4.3 .3 .

\section{Load}

For the three-dimensional models, only one excitation frequency will be analyzed. The load applied on the actuator has, as it was pointed out in Section 4.3.2, a frequency of $200 \mathrm{~Hz}$. A parametric analysis varying the excitation magnitude and frequency will be done in a two-dimensional model in Section 4.3.6. The results from the two-dimensional model can be correlated with the three-dimensional case using the ratios shown in Figure 4.14, as a first approximation.

\section{Boundary Conditions}

The base film is modeled as glued to the substrate where the APF is applied. Therefore, the three translational degrees of freedom are set to zero on the bottom surface of the base film. The two side surfaces (the surfaces that have the $x$ axis a normal, have periodic boundary conditions enforced between them. Lastly, the front and back surfaces ( $z$ axis as normal), have symmetry boundary conditions $(\mathrm{UZ}=\mathrm{ROTX}=\mathrm{ROTY}=0)$.

\section{Mesh}

The mesh is composed of linear, hexahedral, continuum elements. The number of elements varies for each volume fraction $V_{f}$ analyzed (See Table 4.5). All the elements of the mesh use a reduced integration scheme. In the top film this is done to avoid shear locking, using (C3D8R) elements. The base film is meshed with reduced integration, hybrid elements (C3D8RH). Hybrid elements must be used when simulating incompressible materials. ABAQUS ${ }^{\circledR}$ Theory Manual [26, §3.2.4] also suggests that reduced integration elements should be used when modeling incompressible materials at finite strain. The actuator is meshed using reduced integration (C3D8R) elements, because it is an cost-effective method if the solution is expected to be smooth or if the stresses and/or strains in the region are not of concern.

The RVE is partitioned into twelve cells to allow for structured meshing. Along the width of the RVE, the element size tapers towards the sides of the RVE, where the actuator's effect is negligible. The elements of the top film have a very high aspect ratio, something that is not desirable, but they do not cause an error flag during the analysis. The cause, is that the thickness of the top film is extremely small compared to the two other dimensions. Better shaped elements could be obtained by making a finer mesh, which should have a transition within the base film, significantly increasing the computational cost of the analysis. 


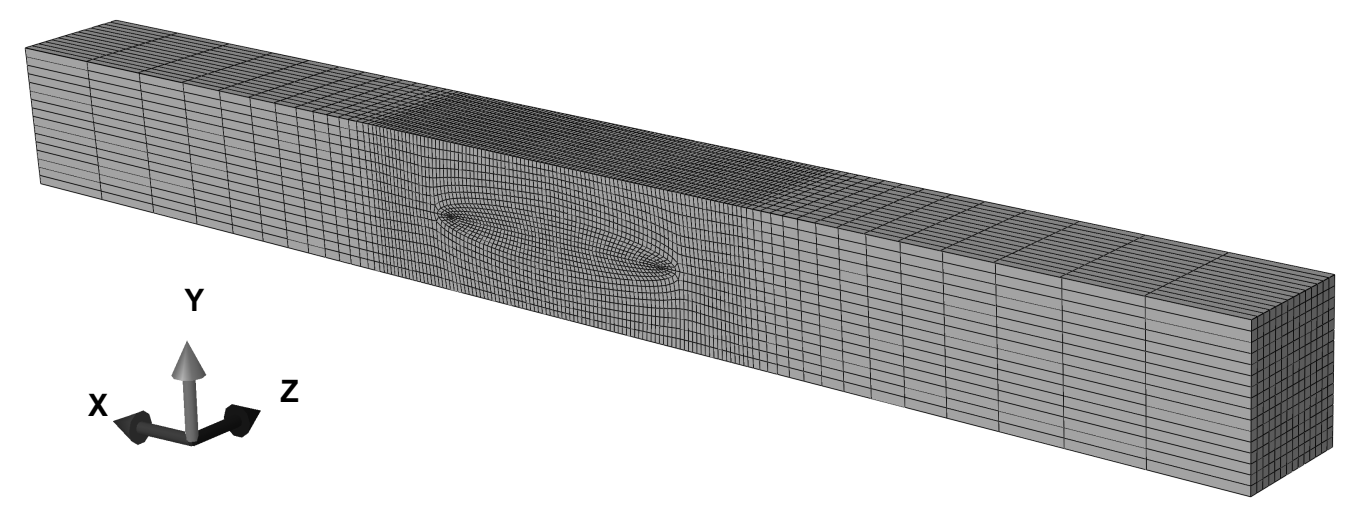

Figure 4.11: Three-dimensional model mesh

\begin{tabular}{|c|c|c|c|c|c|c|}
\hline $\begin{array}{l}\overrightarrow{0} \\
\frac{0}{0} \\
0 \\
\sum\end{array}$ & 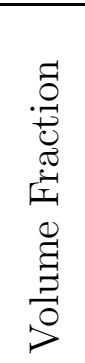 & 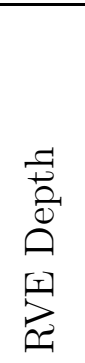 & 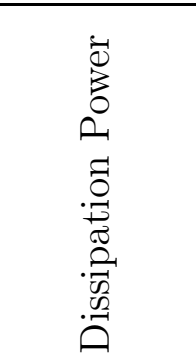 & 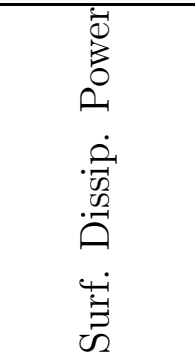 & 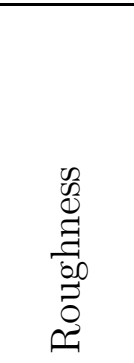 & 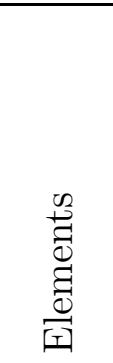 \\
\hline & {$[\%]$} & {$[\mu \mathrm{m}]$} & {$[\mathrm{mW}]$} & {$\left[\mathrm{mW} / \mathrm{mm}^{2}\right]$} & {$[\mu \mathrm{m}]$} & \\
\hline $2 \mathrm{D}-1$ & 5.23 & $\infty$ & $0.012638^{1}$ & 0.0421 & 0.9440 & 13621 \\
\hline $3 \mathrm{D}-1$ & 3.32 & 5.25 & 0.0000496 & 0.0314 & 0.8344 & 12482 \\
\hline $3 \mathrm{D}-2$ & 1.92 & 9.1 & 0.0000310 & 0.0114 & 0.5146 & 16630 \\
\hline $3 \mathrm{D}-3$ & 1.39 & 12.5 & 0.0000294 & 0.0078 & 0.4512 & 25970 \\
\hline $3 \mathrm{D}-4$ & 0.70 & 25 & 0.0000264 & 0.0035 & 0.4487 & 33976 \\
\hline
\end{tabular}

Table 4.5: Results for the two- and three-dimensional diluted models

Figure 4.3 shows the mesh for the deepest $(25 \mu \mathrm{m})$ model.

\section{Results}

The computational effort to solve the three-dimensional models is considerable. For example, the largest model took $3 \mathrm{~h} 10 \mathrm{~min}$ to solve and post-process in a PC system with a 4-core CPU and 4 GB RAM. The dissipation power, dissipation power per unit surface and roughness for these analyses are included in Table 4.5. As the depth of the RVE is increased, the dissipation power per unit area decreases, not only because the neighboring effect is reduced but mostly because the dissipation caused by the actuator is divided by a larger area. In the limit, the surface dissipation tends to zero when the volume fraction goes to zero, as it can be seen in Figure 4.12, In Figure 4.13, it can be seen that although the dissipation power is reduced for lower volume fractions (due to the neighboring effect), the extrapolation to $V_{f}=0$ yields a dissipation power of $2.5 \times 10^{-5} \mathrm{~mW}$. This limiting case could be considered as a single actuator embedded in an infinite medium. 


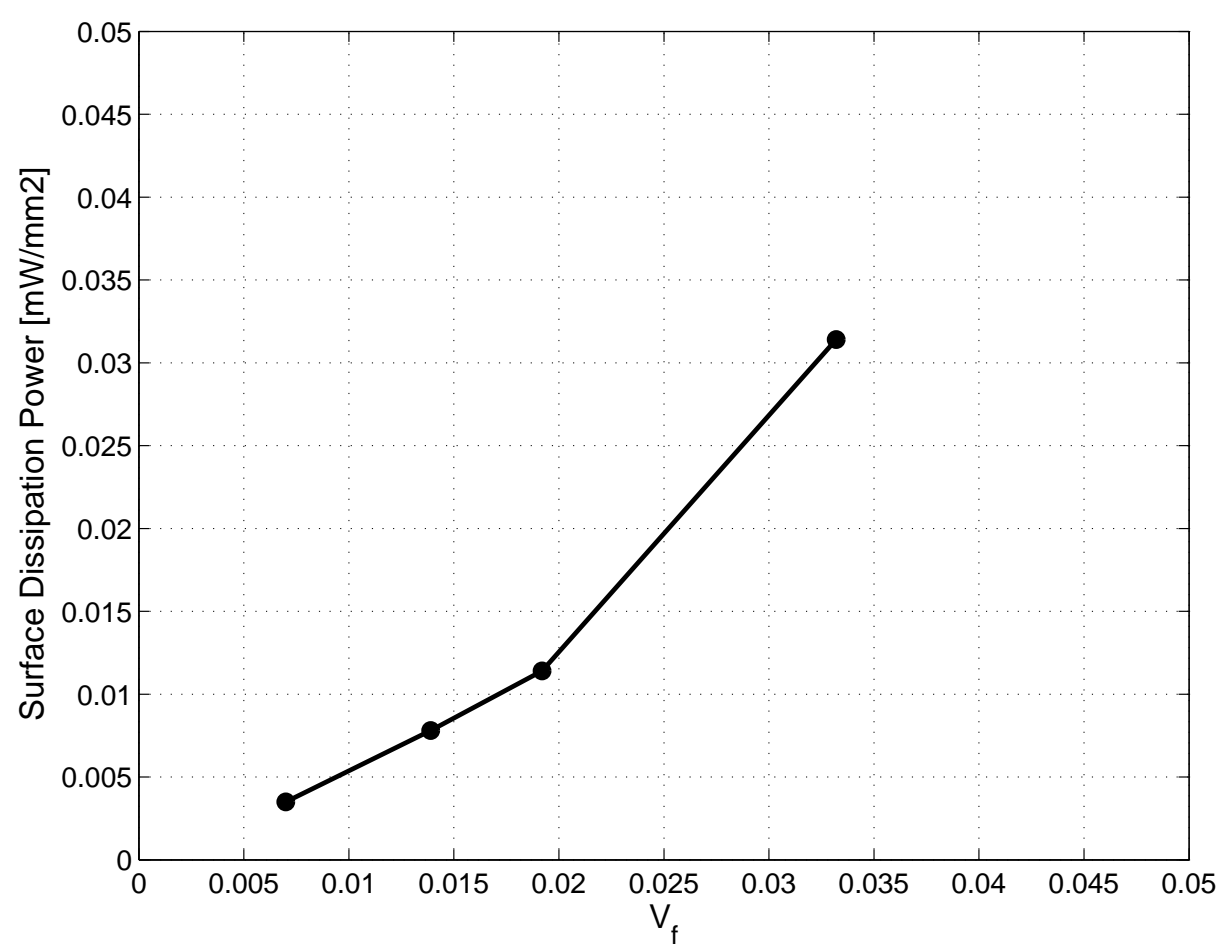

Figure 4.12: Surface dissipation power as a function of the volume fraction for the threedimensional models

\subsubsection{Correlation between $2 \mathrm{D}$ and $3 \mathrm{D}$}

Based on the results from Sections 4.3.3 and 4.3.4, a correlation between the two- and threedimensional response based on the volume fraction $V_{f}$ is proposed. For the two-dimensional model the volume fraction $V_{f}$ is equal to the area fraction $A_{f}$, because the model is assumed to be in plane strain. The surface dissipation power and roughness from the two-dimensional model are set as the reference values and the ratios of three-dimensional to two-dimensional results are calculated as a function of the volume fraction, as follows:

$$
r_{[\bullet]}\left(V_{f}\right)=\frac{[\bullet]_{3 \mathrm{D}}\left(V_{f}\right)}{[\bullet]_{2 \mathrm{D}}\left(A_{f}\right)}
$$

The calculated ratios $r_{R}, r_{D}$ for the roughness and dissipation, respectively, are included in Figure 4.14.

It is important to bear in mind that these results were calculated for a sinusoidal load with $10 \mathrm{MPa}$ amplitude and $200 \mathrm{~Hz}$ frequency for an RVE width of $300 \mu \mathrm{m}$, resulting in $A_{f}=V_{f}=5.23 \%$ for the two-dimensional case.

\footnotetext{
${ }^{1}$ Because this result is from a plane strain model, the units are per mm depth. Thus, they should not be compared with the other results under this column.
} 


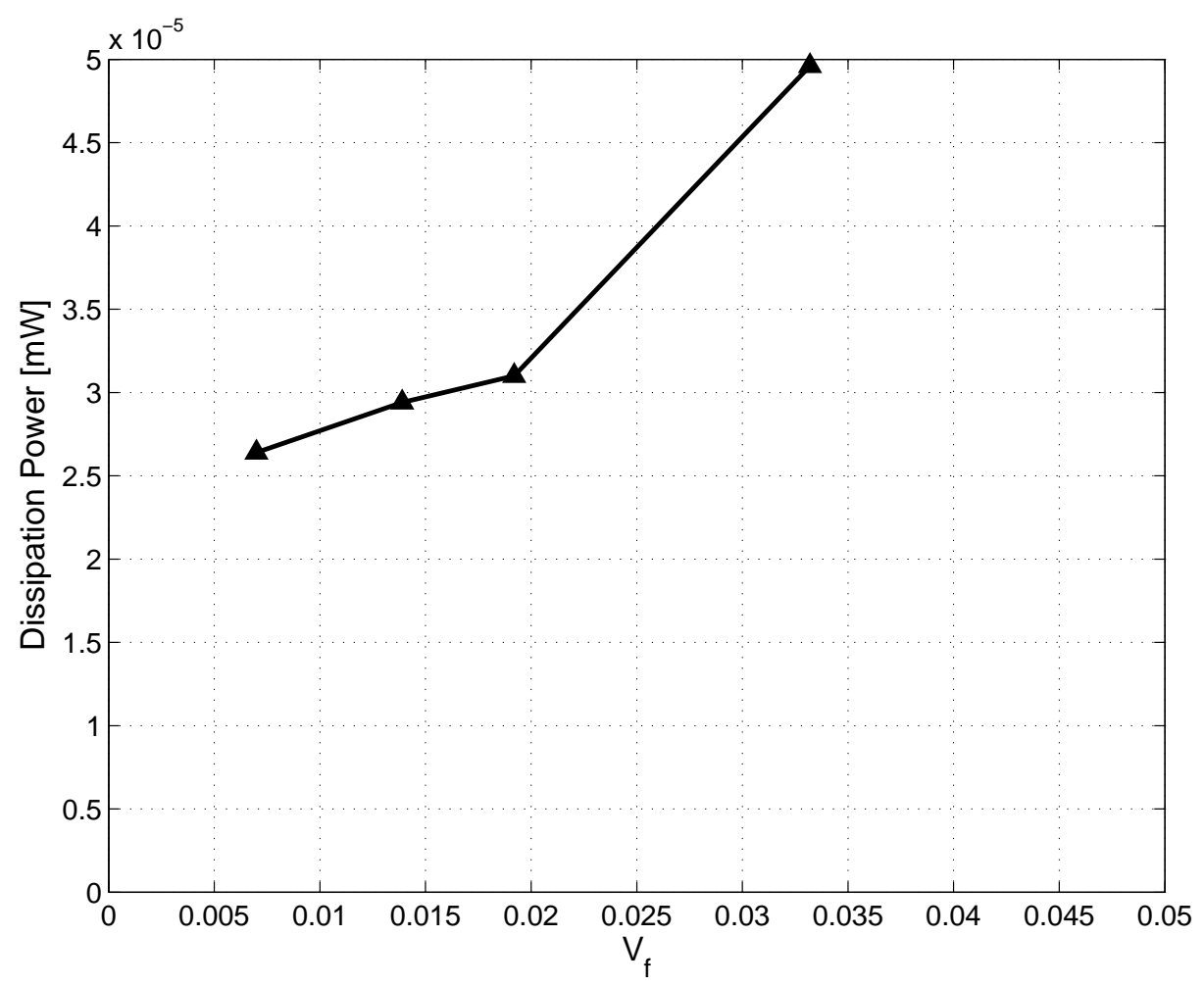

Figure 4.13: Dissipation power as a function of the volume fraction for the three-dimensional models

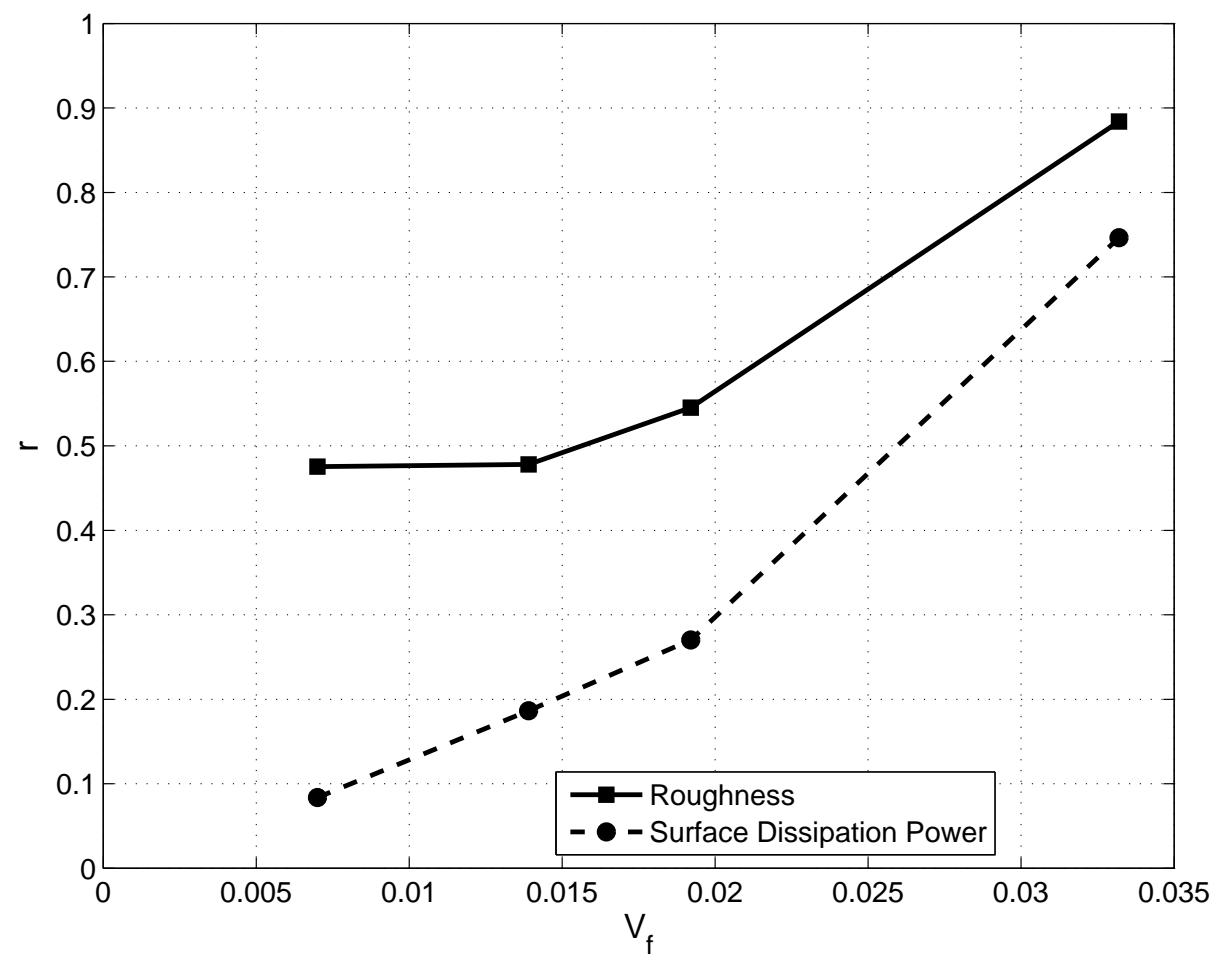

Figure 4.14: Ratios for roughness and surface dissipation power 


\subsubsection{Two Dimensional - Parametric Analysis}

In this section a two-dimensional parametric analysis is done on a RVE with one actuator. Because periodic boundary conditions are enforced, it is asssumed that all the actuators are coherent or polarized (i.e. all the right tips will go up and all the left tips will go down). The problem with two neighboring actuators having opposite polarization has been treated in Section 4.2 ,

The parameters that will be analyzed are: frequency and magnitude of the excitation, volume fraction (changing the width of the film), vertical location of the actuator within the base film, aspect ratio of the actuator and the Young's modulus of the top film and the actuator. Also a mesh dependency analysis will be done to show that the results are convergent.

The model 2D-P-01 in Table 4.6 is set as a reference for comparison purposes in the parametric analyses.

The PYTHON script used to analyze these models is the same as in Section 4.3.3, which is listed in the Appendix E.2.

All the results of this section are listed in Table 4.6.

\section{Excitation Frequency}

Here, a fixed width $(120 \mu \mathrm{m})$ of the RVE will be considered. The frequency of the excitation will be changed from $20 \mathrm{~Hz}$ to $200 \mathrm{~Hz}$ in increments of $30 \mathrm{~Hz}$. The results are shown in Figures 4.15 and 4.16, and they are based on the models 2D-P-01 to 2D-P-07.

\section{Torque Magnitude}

As the torque generated by actuators depends on the intensity of the electric field applied, and the magnitude of the torque has not been experimentally determined yet (see Section 6.1), a parametric study will be done to assess its influence on the APF. In the present work, the torque generated by the actuators is materialized by the application of a surface traction with the same magnitude but opposite directions in the top and bottom surfaces of the actuators. Here, the magnitude of the traction will be increased and reduced to study the response of the APF. Being the torque proportional to the applied traction, if the latter is changed, the former will also change accordingly.

The results are shown in Figures 4.17 and 4.18, The models used to create these figures are 2D-P-01, 2D-P-20 and 2D-P-21, which are listed in Table 4.6. As it can be seen in these figures, the response is non-linear, as it was expected because the base film material is not linear (see Figure 4.1).

\section{Actuator Location}

In this Section the vertical location of the ellipse is modified and the response is analyzed. Figures 4.19 and 4.20 show the results of the models 2D-P-01, 2D-P-08 and 2D-P-09. It is noticeable how, both surface dissipation and roughness, increase as the actuator is closer to the top surface. If the roughness needs to be increased and power consumption is not a concern, moving the actuators towards the top surface could be a good option. 


\begin{tabular}{|c|c|c|c|c|c|c|c|c|c|c|c|c|}
\hline $\begin{array}{l}\overrightarrow{0} \\
\frac{0}{0} \\
\stackrel{2}{z}\end{array}$ & 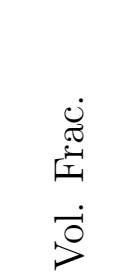 & 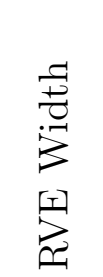 & 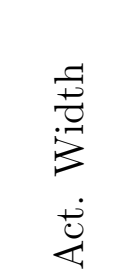 & 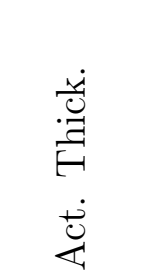 & 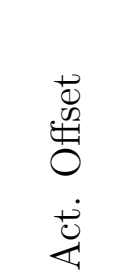 & 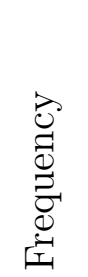 & 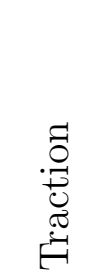 & 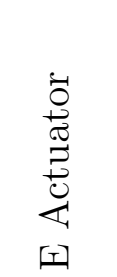 & 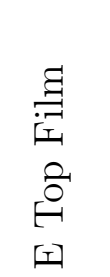 & 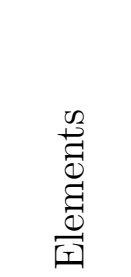 & 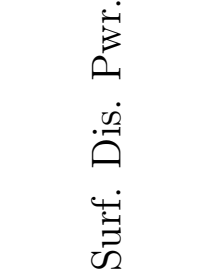 & 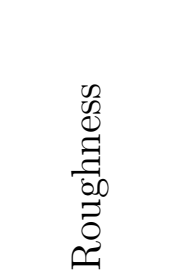 \\
\hline & & {$[\mathrm{mm}]$} & {$[\mathrm{mm}]$} & {$[\mathrm{mm}]$} & {$[\mathrm{mm}]$} & {$[\mathrm{Hz}]$} & {$[\mathrm{MPa}]$} & {$[\mathrm{GPa}]$} & {$[\mathrm{GPa}]$} & & {$\left[\mathrm{mW} / \mathrm{mm}^{2}\right]$} & {$[\mathrm{mm}]$} \\
\hline $2 \mathrm{D}-\mathrm{P}-01$ & 0.1308 & 0.12 & 0.0600 & 0.0100 & 0.000 & 200 & 10 & 3 & 70 & 8040 & 0.1072 & 0.000959 \\
\hline 2D-P-02 & 0.1308 & 0.12 & 0.0600 & 0.0100 & 0.000 & 170 & 10 & 3 & 70 & 8040 & 0.0961 & 0.000980 \\
\hline 2D-P-03 & 0.1308 & 0.12 & 0.0600 & 0.0100 & 0.000 & 140 & 10 & 3 & 70 & 8040 & 0.0842 & 0.001005 \\
\hline 2D-P-04 & 0.1308 & 0.12 & 0.0600 & 0.0100 & 0.000 & 110 & 10 & 3 & 70 & 8040 & 0.0709 & 0.001038 \\
\hline 2D-P-05 & 0.1308 & 0.12 & 0.0600 & 0.0100 & 0.000 & 80 & 10 & 3 & 70 & 8040 & 0.0560 & 0.001083 \\
\hline 2D-P-06 & 0.1308 & 0.12 & 0.0600 & 0.0100 & 0.000 & 50 & 10 & 3 & 70 & 8040 & 0.0388 & 0.001151 \\
\hline 2D-P-07 & 0.1308 & 0.12 & 0.0600 & 0.0100 & 0.000 & 20 & 10 & 3 & 70 & 8040 & 0.0179 & 0.001285 \\
\hline 2D-P-08 & 0.1308 & 0.12 & 0.0600 & 0.0100 & -0.005 & 200 & 10 & 3 & 70 & 8809 & 0.0268 & 0.000327 \\
\hline 2D-P-09 & 0.1308 & 0.12 & 0.0600 & 0.0100 & 0.005 & 200 & 10 & 3 & 70 & 8426 & 0.1935 & 0.001904 \\
\hline 2D-P-10 & 0.1744 & 0.09 & 0.0600 & 0.0100 & 0.000 & 200 & 10 & 3 & 70 & 4428 & 0.1647 & 0.001084 \\
\hline 2D-P-11 & 0.1046 & 0.15 & 0.0600 & 0.0100 & 0.000 & 200 & 10 & 3 & 70 & 5292 & 0.0829 & 0.000940 \\
\hline 2D-P-12 & 0.0523 & 0.30 & 0.0600 & 0.0100 & 0.000 & 200 & 10 & 3 & 70 & 13621 & 0.0421 & 0.000944 \\
\hline 2D-P-13 & 0.1308 & 0.12 & 0.0600 & 0.0100 & 0.000 & 200 & 10 & 3 & 70 & 1931 & 0.0951 & 0.000917 \\
\hline 2D-P-14 & 0.1308 & 0.12 & 0.0600 & 0.0100 & 0.000 & 200 & 10 & 3 & 70 & 17394 & 0.1072 & 0.000959 \\
\hline 2D-P-15 & 0.1308 & 0.12 & 0.0600 & 0.0100 & 0.000 & 200 & 10 & 3 & 70 & 4915 & 0.1052 & 0.000958 \\
\hline 2D-P-16 & 0.0824 & 0.12 & 0.0238 & 0.0159 & 0.000 & 200 & 10 & 3 & 70 & 4101 & 0.0238 & 0.015874 \\
\hline $2 \mathrm{D}-\mathrm{P}-17$ & 0.1602 & 0.12 & 0.0900 & 0.0082 & 0.000 & 200 & 10 & 3 & 70 & 4239 & 0.0274 & 0.000622 \\
\hline 2D-P-18 & 0.1308 & 0.12 & 0.0600 & 0.0100 & 0.000 & 200 & 10 & 3 & 70 & 8830 & 0.1059 & 0.000956 \\
\hline 2D-P-19 & 0.1308 & 0.12 & 0.0600 & 0.0100 & 0.000 & 200 & 10 & 3 & 70 & $8830^{1}$ & 0.1078 & 0.000962 \\
\hline 2D-P-20 & 0.1308 & 0.12 & 0.0600 & 0.0100 & 0.000 & 200 & 5 & 3 & 70 & 8830 & 0.0089 & 0.000377 \\
\hline 2D-P-21 & 0.1308 & 0.12 & 0.0600 & 0.0100 & 0.000 & 200 & 15 & 3 & 70 & 8830 & 0.3405 & 0.001704 \\
\hline 2D-P-22 & 0.1308 & 0.12 & 0.0600 & 0.0100 & 0.000 & 200 & 10 & 2 & 70 & 8830 & 0.1084 & 0.000968 \\
\hline $2 \mathrm{D}-\mathrm{P}-23$ & 0.1308 & 0.12 & 0.0600 & 0.0100 & 0.000 & 200 & 10 & 1 & 70 & 8830 & 0.1168 & 0.000990 \\
\hline 2D-P-24 & 0.1308 & 0.12 & 0.0600 & 0.0100 & 0.000 & 200 & 10 & 3 & 50 & 8830 & 0.1061 & 0.000954 \\
\hline $2 \mathrm{D}-\mathrm{P}-25$ & 0.1308 & 0.12 & 0.0600 & 0.0100 & 0.000 & 200 & 10 & 3 & 30 & 8830 & 0.1065 & 0.000950 \\
\hline 2D-P-26 & 0.1308 & 0.12 & 0.0600 & 0.0100 & 0.000 & 200 & 10 & 3 & 30 & $9070^{2}$ & 0.1065 & 0.000950 \\
\hline
\end{tabular}

Table 4.6: Results for the two-dimensional parametric models

\footnotetext{
${ }^{a}$ This model has been solved using quadratic elements (CPE8R and CPE8RH).
}

${ }^{b}$ Two elements were used through the thickness of the top film in this model. 


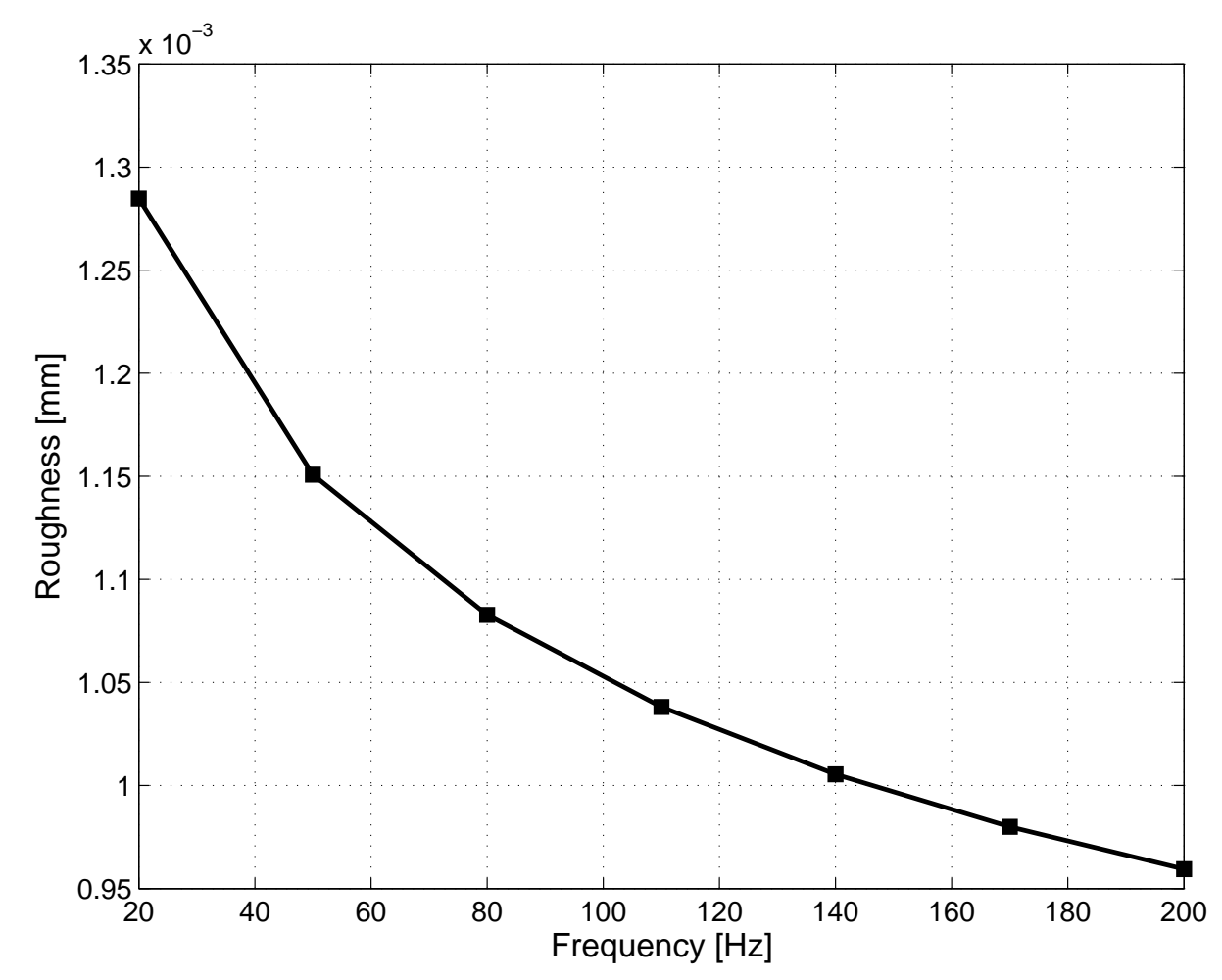

Figure 4.15: Roughness as a function of the excitation frequency

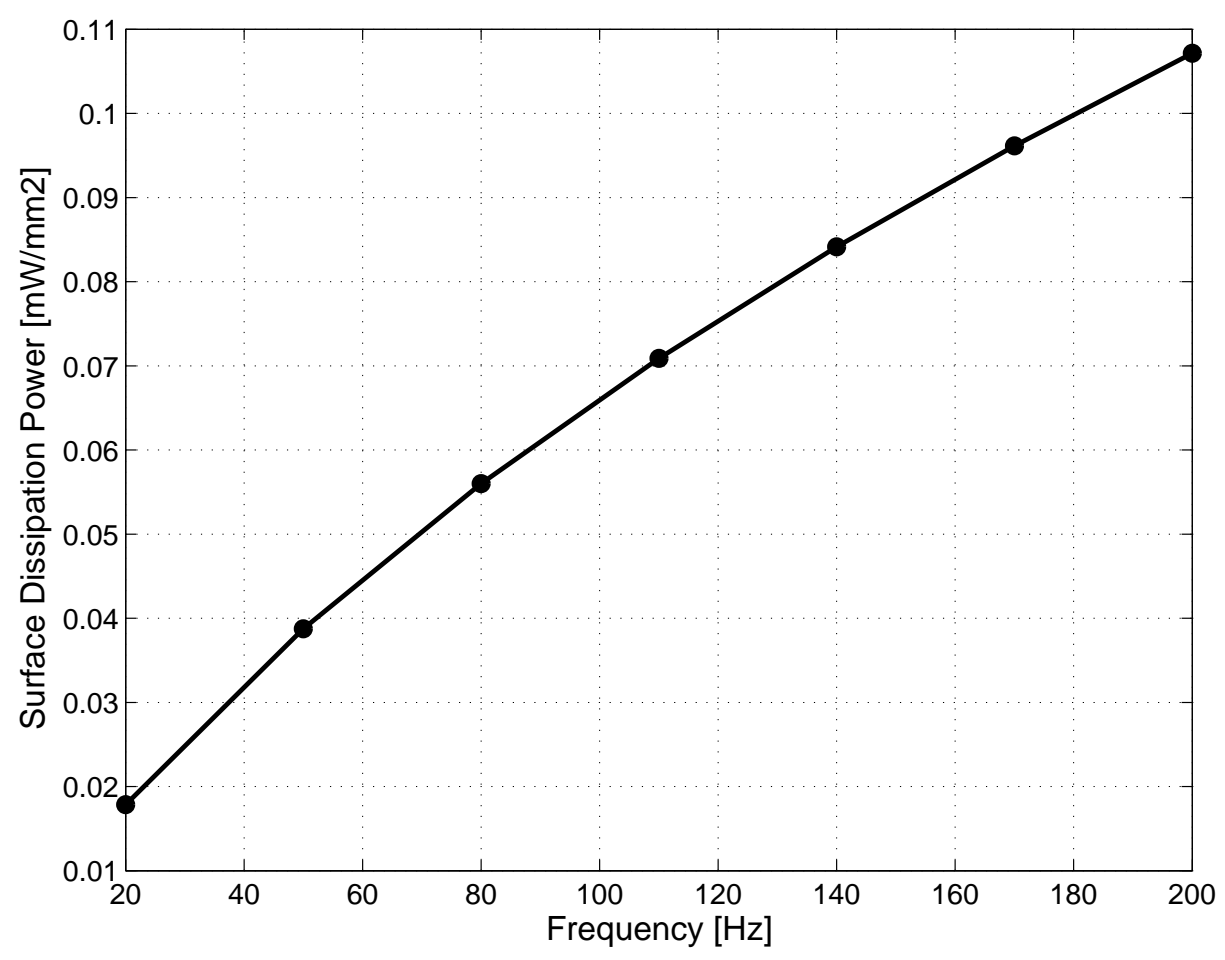

Figure 4.16: Surface Dissipation Power as a function of the excitation frequency 


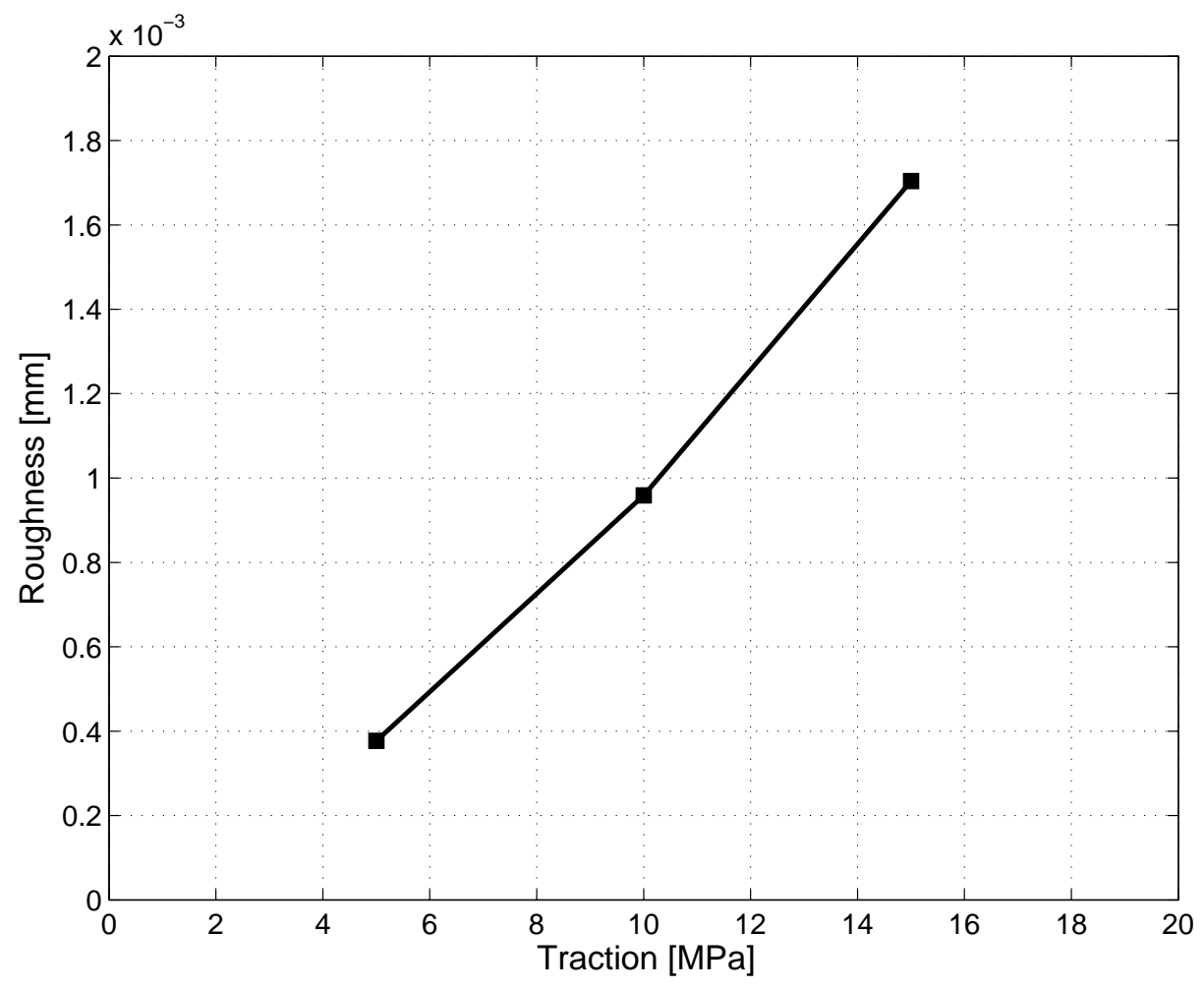

Figure 4.17: Roughness as a function of the surface traction magnitude

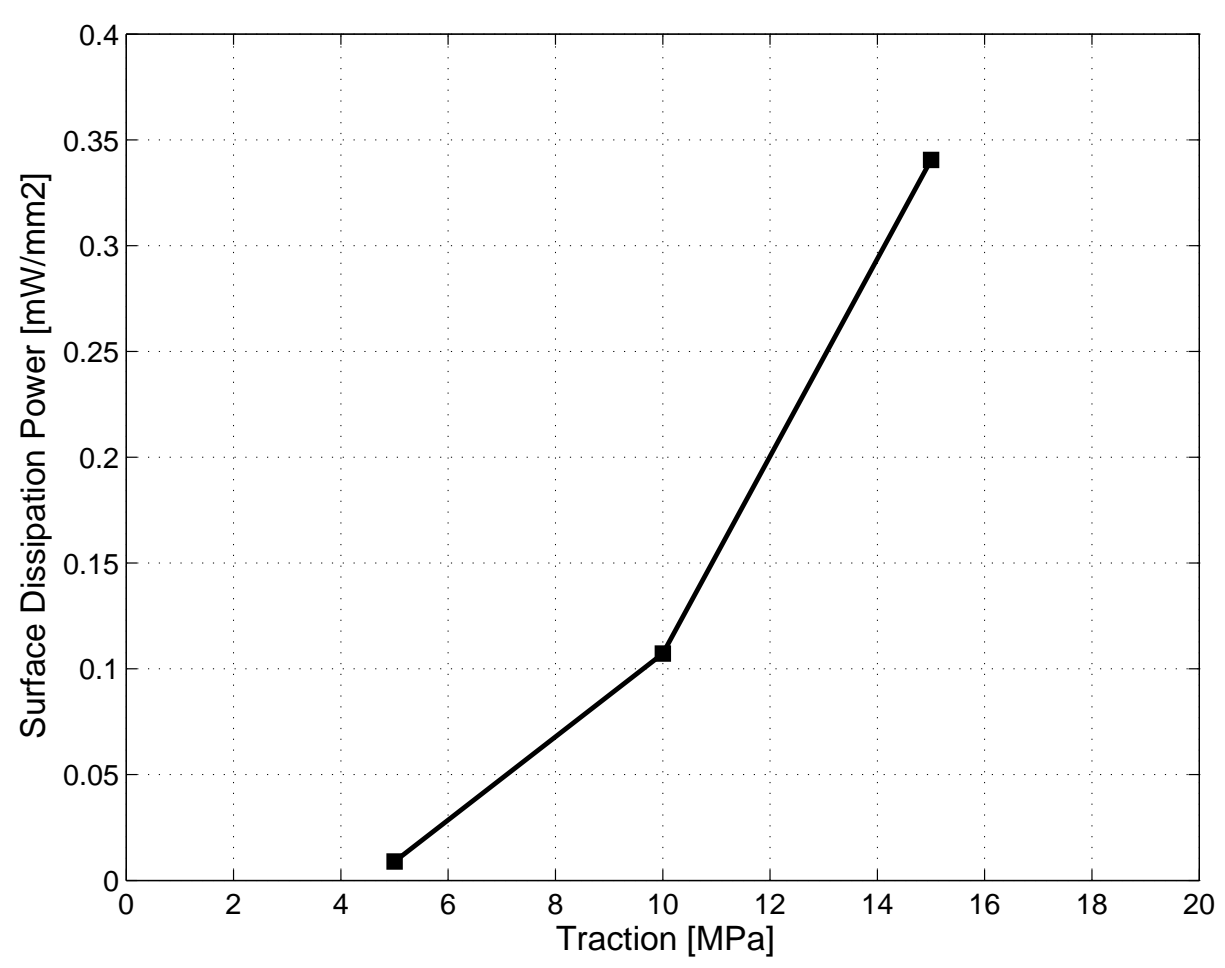

Figure 4.18: Surface dissipation power as a function of the surface traction magnitude 


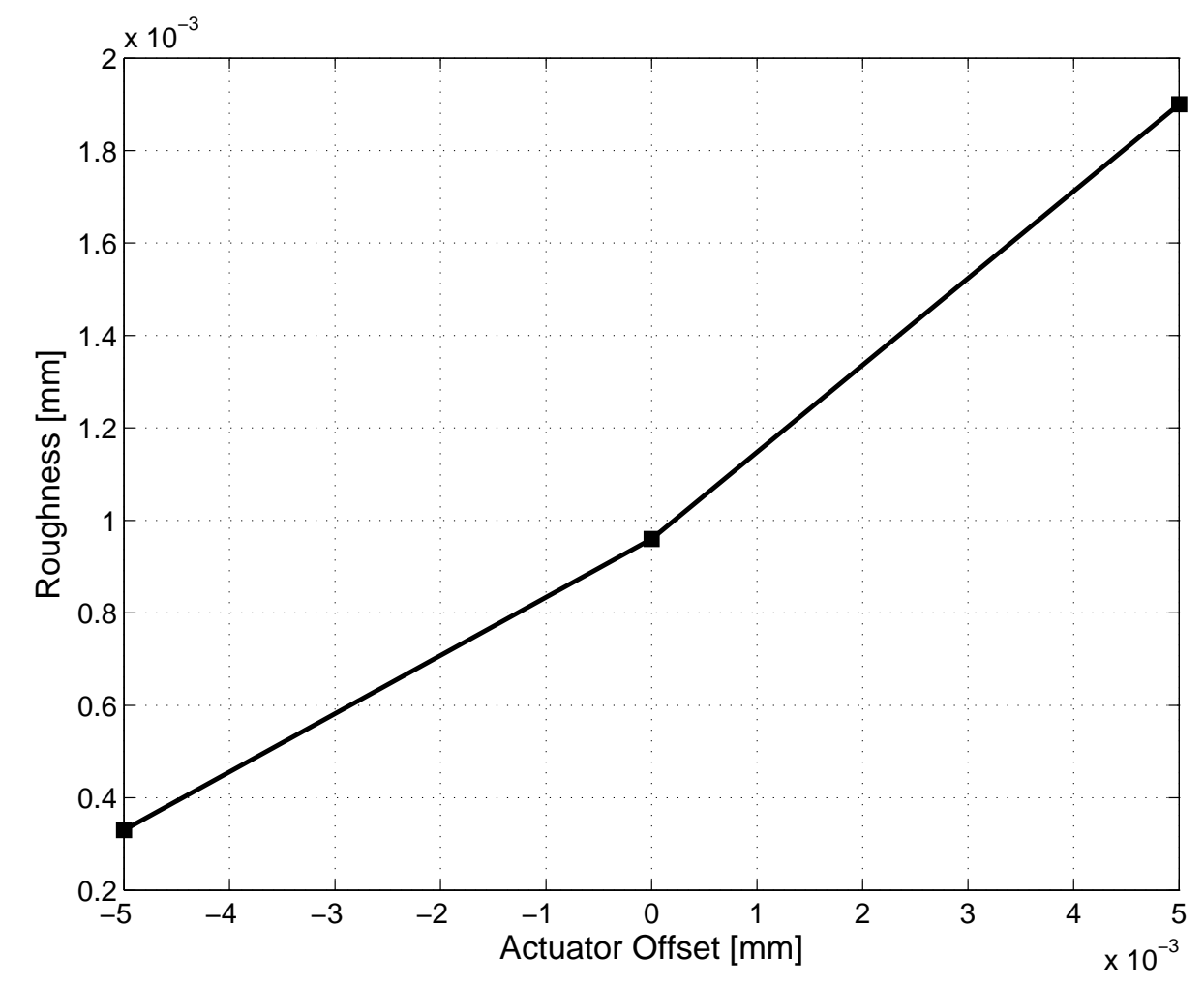

Figure 4.19: Roughness as a function of the actuator's vertical offset

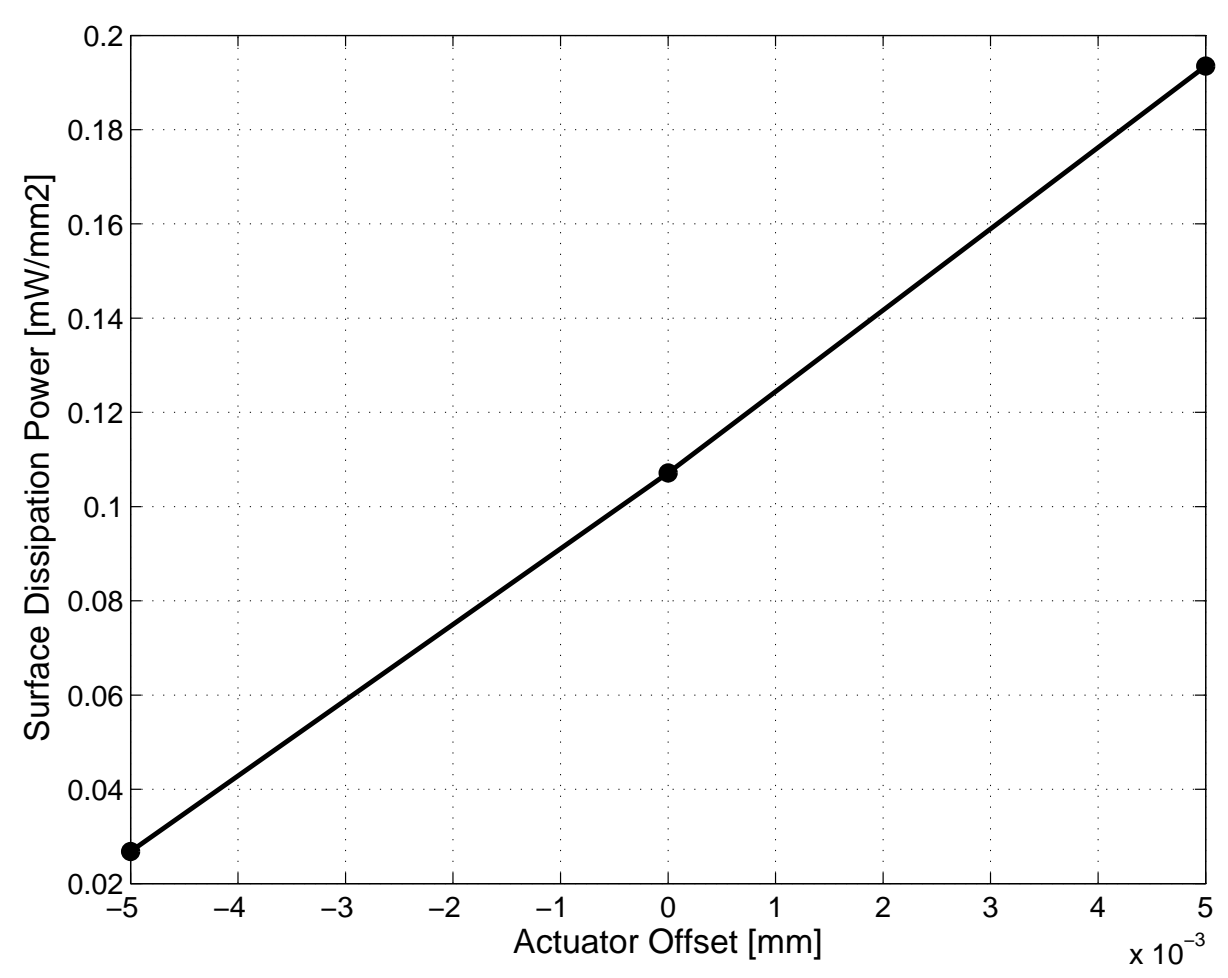

Figure 4.20: Surface Dissipation Power as a function of the actuator's vertical offset 


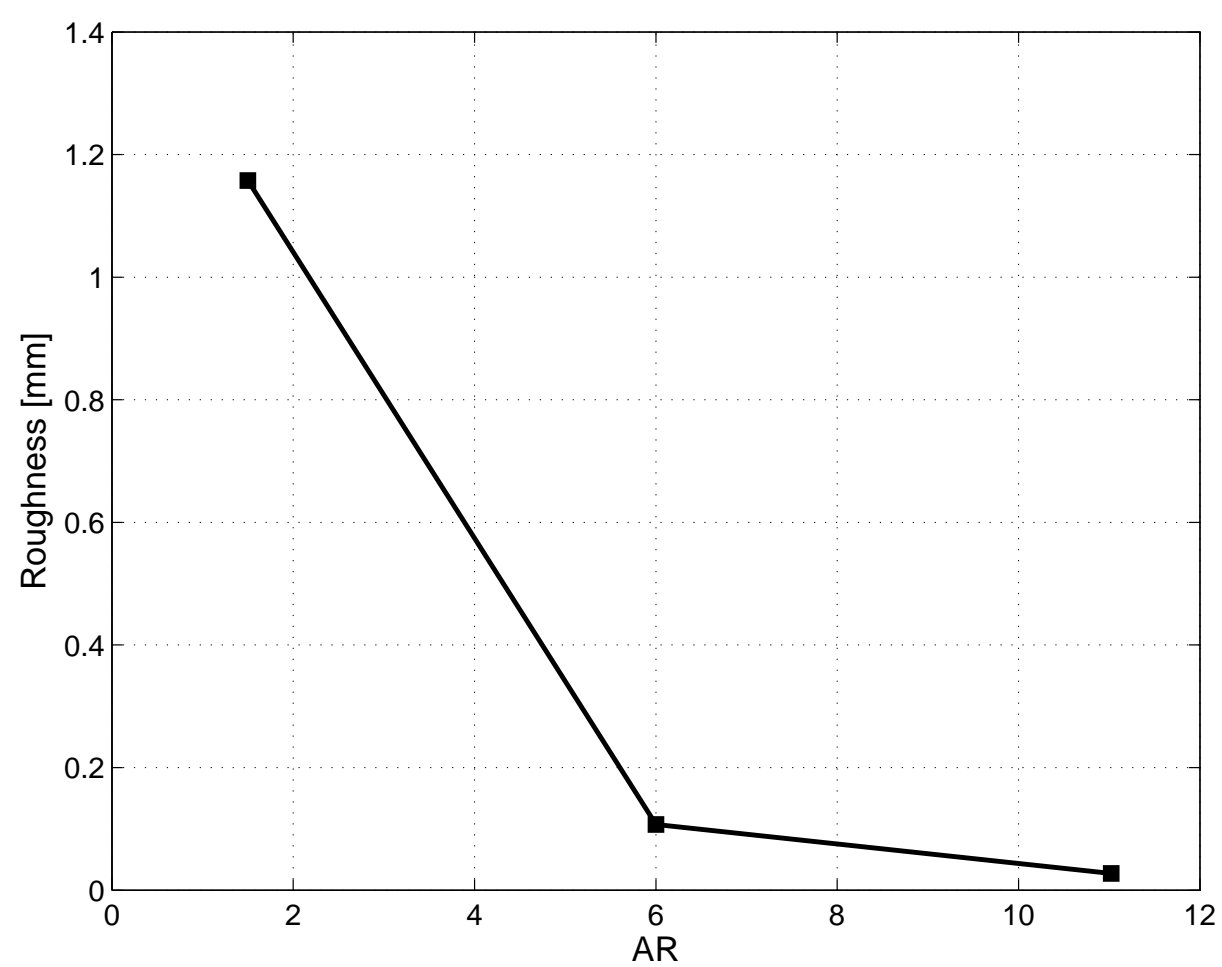

Figure 4.21: Roughness as a function of the actuator's aspect ratio

\section{Actuator Geometry}

In this Section the geometry of the actuator is changed and the response is analyzed. Three models (2D-P-01, 2D-P-16 and 2D-P-17) are created where the geometry of the actuator is changed under the constraint of keeping the volume of the ellipsoid constant (not the area). The roughness and surface dissipation power are plotted against the actuator's aspect ratio AR in Figures 4.21 and 4.22, respectively. The aspect ratio is defined as,

$$
\mathrm{AR}=\frac{c}{a}
$$

where $c, a$ are the major and minor radii, respectively. And the volume of a prolate spheroid is given by,

$$
\mathrm{Vol}=\frac{4}{3} \pi a^{2} c
$$

An effort to analyze actuators with aspect ratio equal or close to 1 (circle) was done without results. The mesh gets highly distorted and the solver does not converge. A possible solution, beyond the scope of the present work, could be a coupled Eulerian-Lagrangian analysis [25, §13.1.1].

\section{Volume Fraction}

To study the effect of the volume fraction $V_{f}$, the width of the RVE is changed and the response is analyzed. The results from models 2D-P-01 and 2D-P-10 to 2D-P-12 are plotted 


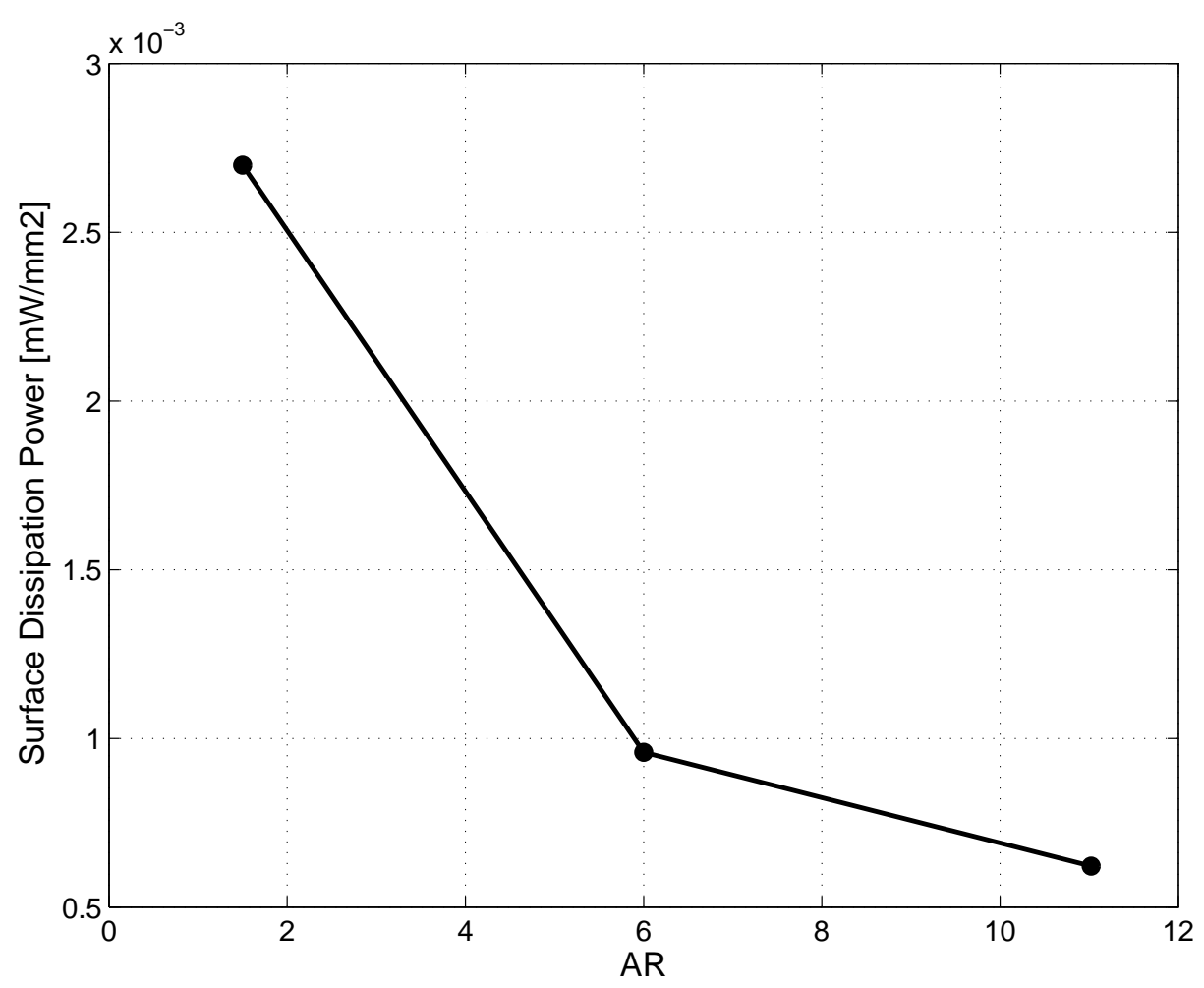

Figure 4.22: Surface Dissipation Power as a function of the actuator's aspect ratio

in Figures 4.23 and 4.24 .

Note that the roughness as defined in this work, is a local measure of the deformation around the actuator. Therefore, the increase of the roughness with the volume fraction shown in Figure 4.23, shows the interaction effect with the neighboring actuators.

\section{Actuator and Top Film Modulus}

In this Section, the actuator and top film Young's modulus will be changed and the impact on the response will be assessed. In the models 2D-P-22 and 2D-P-23, the actuator's modulus will be set to $2000 \mathrm{MPa}$ and $1000 \mathrm{MPa}$, respectively, while keeping all the other parameters equal to the ones of the reference model 2D-P-01. The top film modulus will be reduced from $70000 \mathrm{MPa}$ to $50000 \mathrm{MPa}$ and $30000 \mathrm{MPa}$ in the models 2D-P-24 and 2D-P-25, respectively. For the two latter models, all the other parameters are the same as in model 2D-P-01.

The surface dissipation power and roughness results for all the aforementioned models are included in Table 4.6 and shown in Figures 4.25 to 4.28. As it can be seen in these figures, the impact on the results of changes in the order of $300 \%$ in the actuator Young's modulus and $233 \%$ in the top film Young's modulus is very small. The most noticeable change is in the surface dissipation power, when the actuators Young's modulus is reduced to $1000 \mathrm{MPa}$, as it is shown in Figure 4.26. The change in the response due to changes in the top film modulus are negligible (see Figures 4.27) and 4.28). This latter fact might happen because the top film, although it has a higher modulus than the base film, it is 1000 times thinner; and the bending stiffness of a plate is proportional to its thickness cubed. 


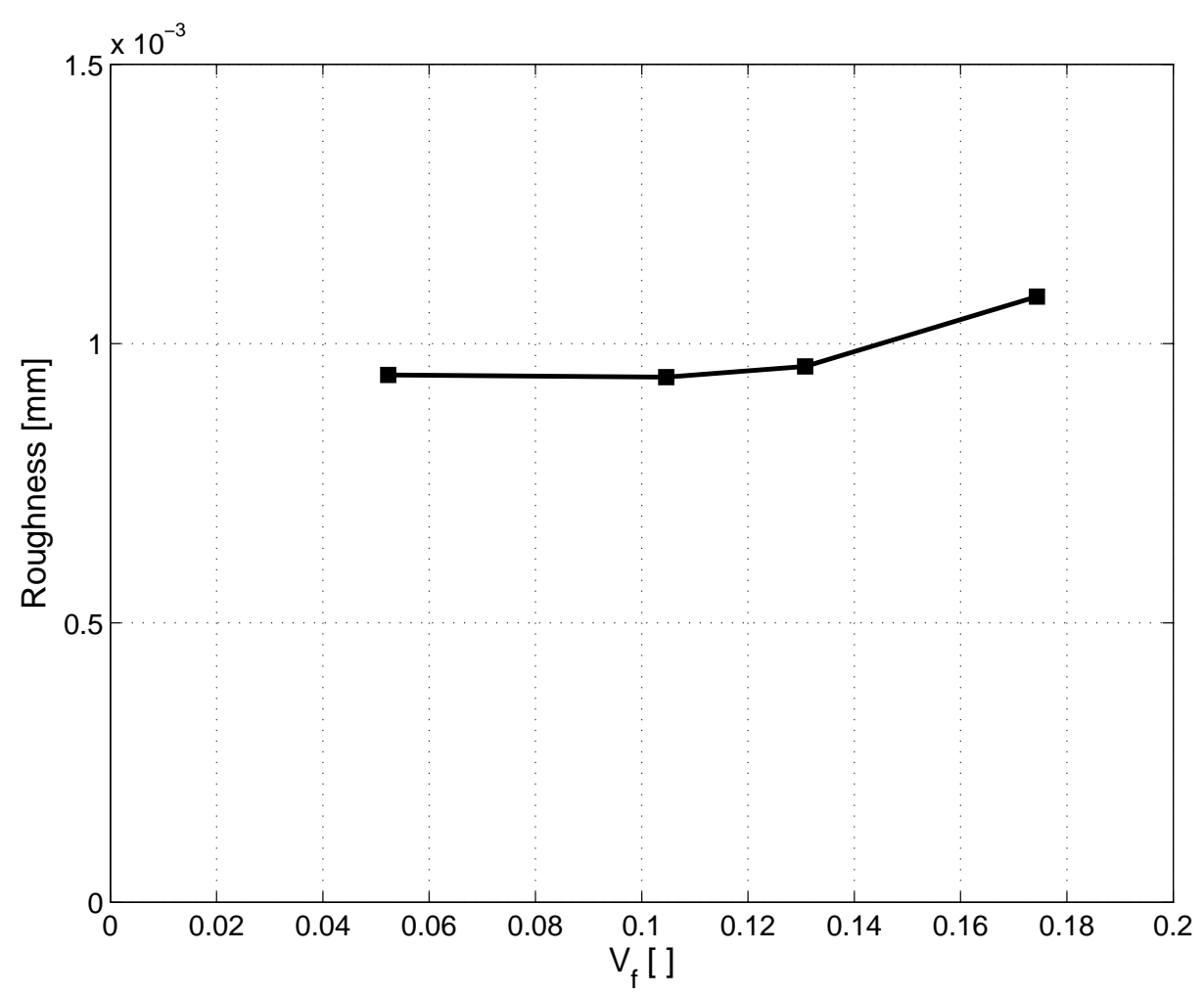

Figure 4.23: Roughness as a function of the volume fraction

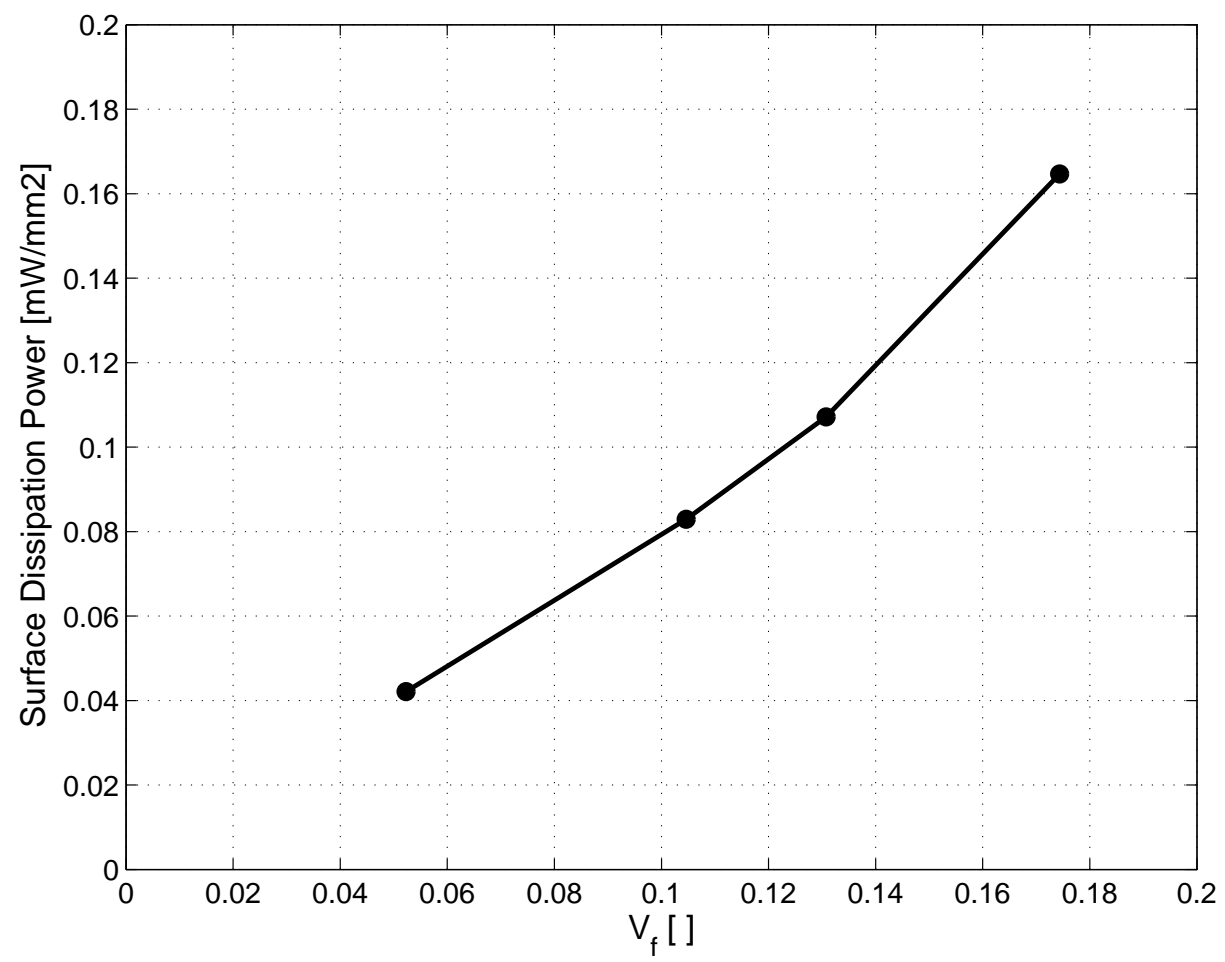

Figure 4.24: Surface Dissipation Power as a function of the volume fraction 


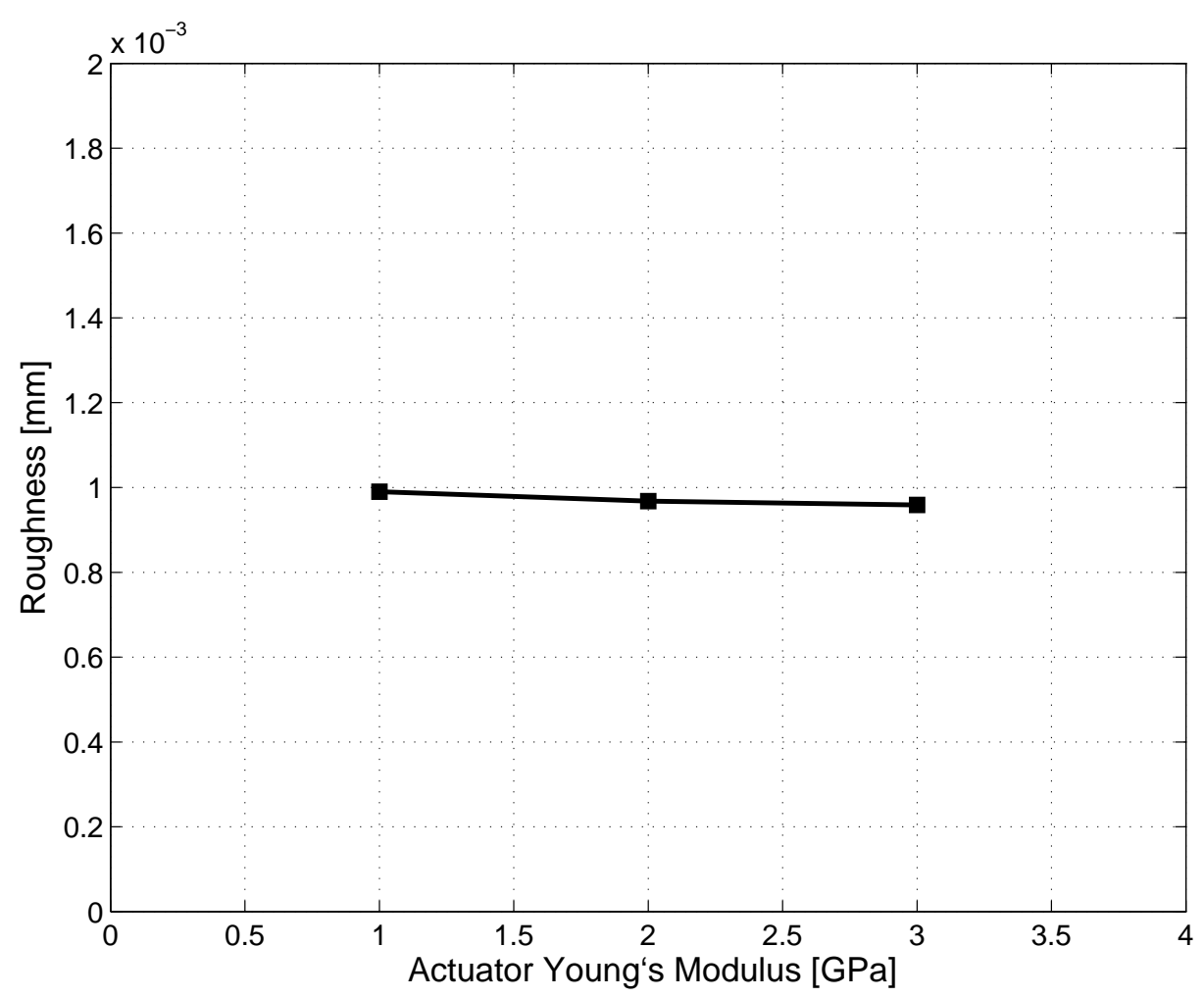

Figure 4.25: Roughness as a function of the actuator Young's modulus

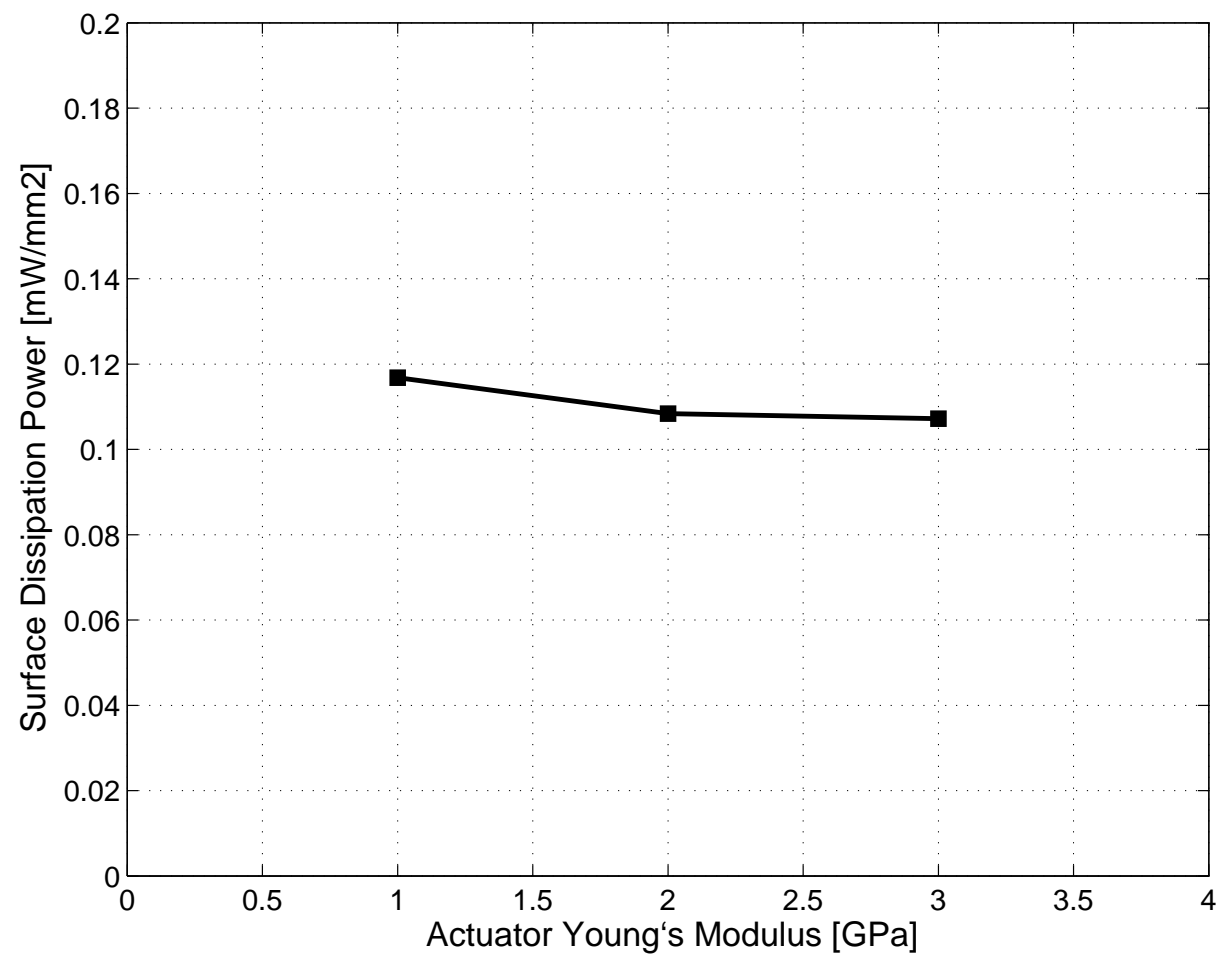

Figure 4.26: Surface dissipation power as a function of the actuator Young's modulus 


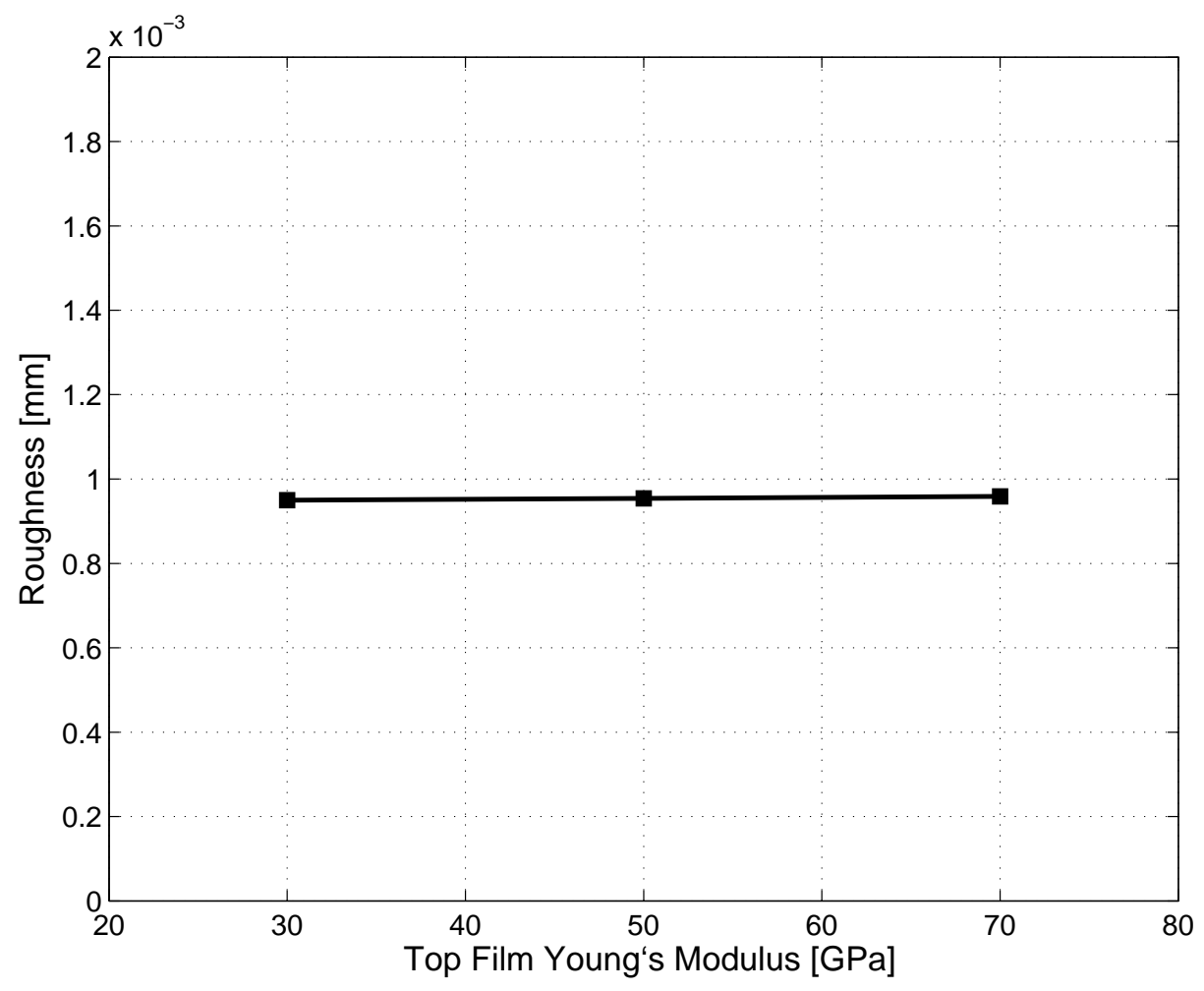

Figure 4.27: Roughness as a function of the top film Young's modulus

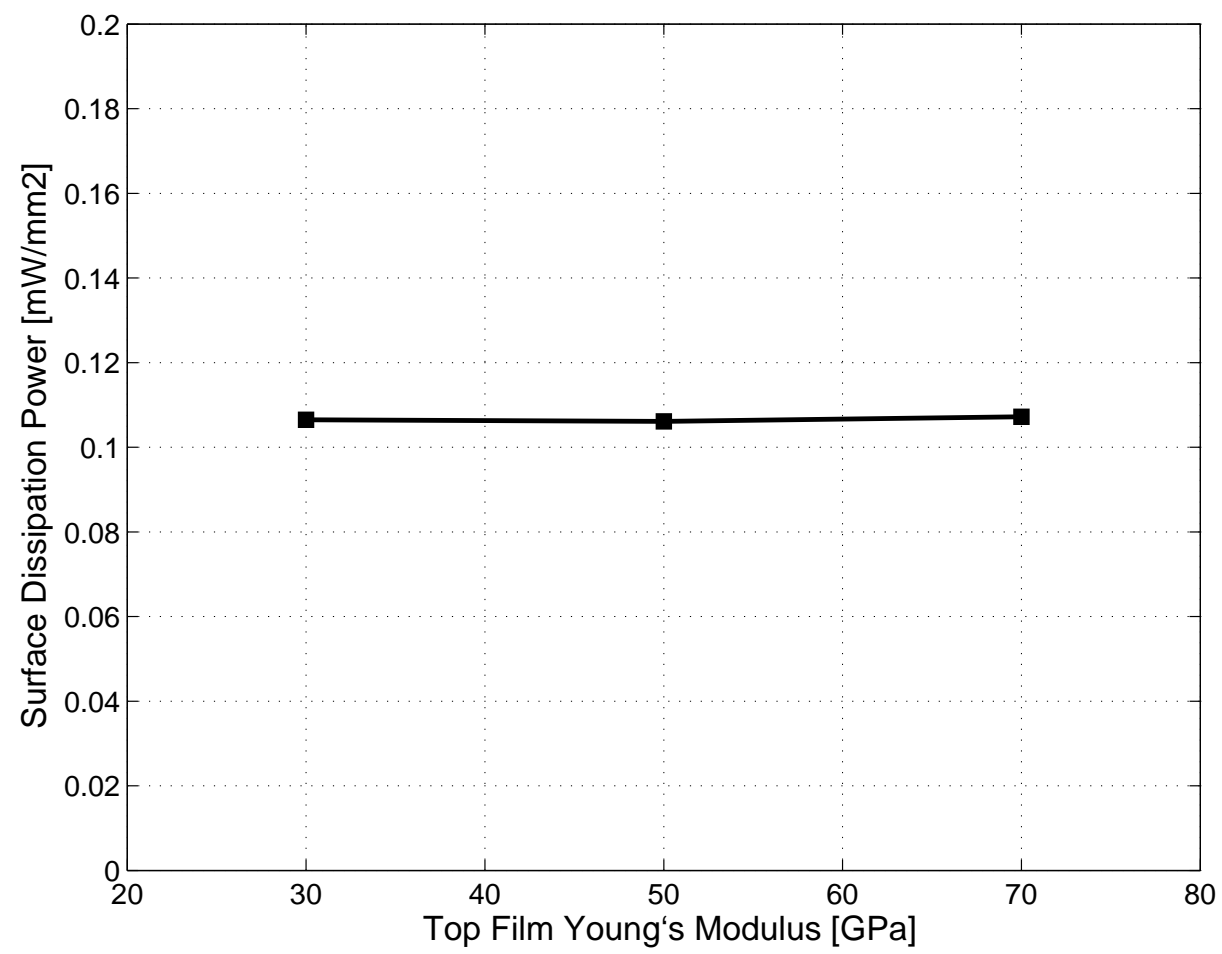

Figure 4.28: Surface dissipation power as a function of the top film Young's modulus 


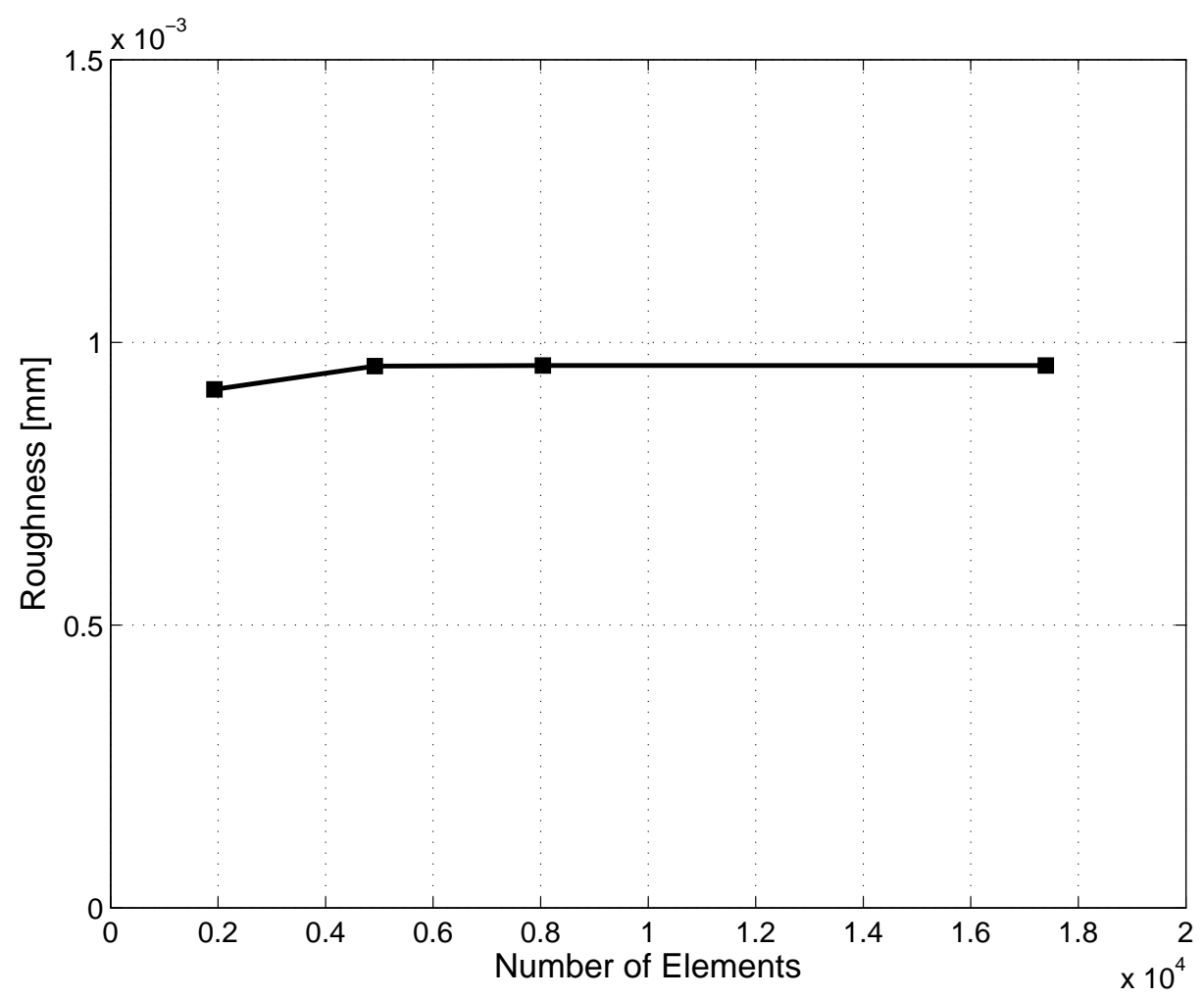

Figure 4.29: Roughness as a function of the number of elements in the mesh

\section{Mesh Dependency Study}

h-Refinement In the h-refinement method, the same type of elements is used, but the size of the elements is changed [50]. For this case, complete regeneration will be used. From a computational point of view this is very expensive, but for this academic problem it is feasible to do it.

Meshes with different element sizes will be generated and the results will be compared. If the problem is mesh independent, the results should converge asymptotically as the element size is reduced.

In Figures 4.29 and 4.30, it can be seen that the results are very similar when the number of elements is increased from 8040 to 17394 . The aforementioned figures are based on the results of models 2D-P-01, 2D-P-13, 2D-P-14 and 2D-P-15.

p-Refinement The idea behind the p-refinement method is to keep the original mesh, but the degree of the elements is changed. ABAQUS ${ }^{\circledR}$ has to types of elements available, linear and quadratic. In order to compare the results, two models are analyzed; one with linear (4 noded), plane strain continuum elements and the other with quadratic (8 noded) elements. The ratio between the roughness and surface dissipation power between both meshes are 1.0063 and 1.0176, respectively. The results for these models are listed in Table 4.6 as 2D-P-18 and 2D-P-19. Being the latter the one meshed with quadratic elements. 


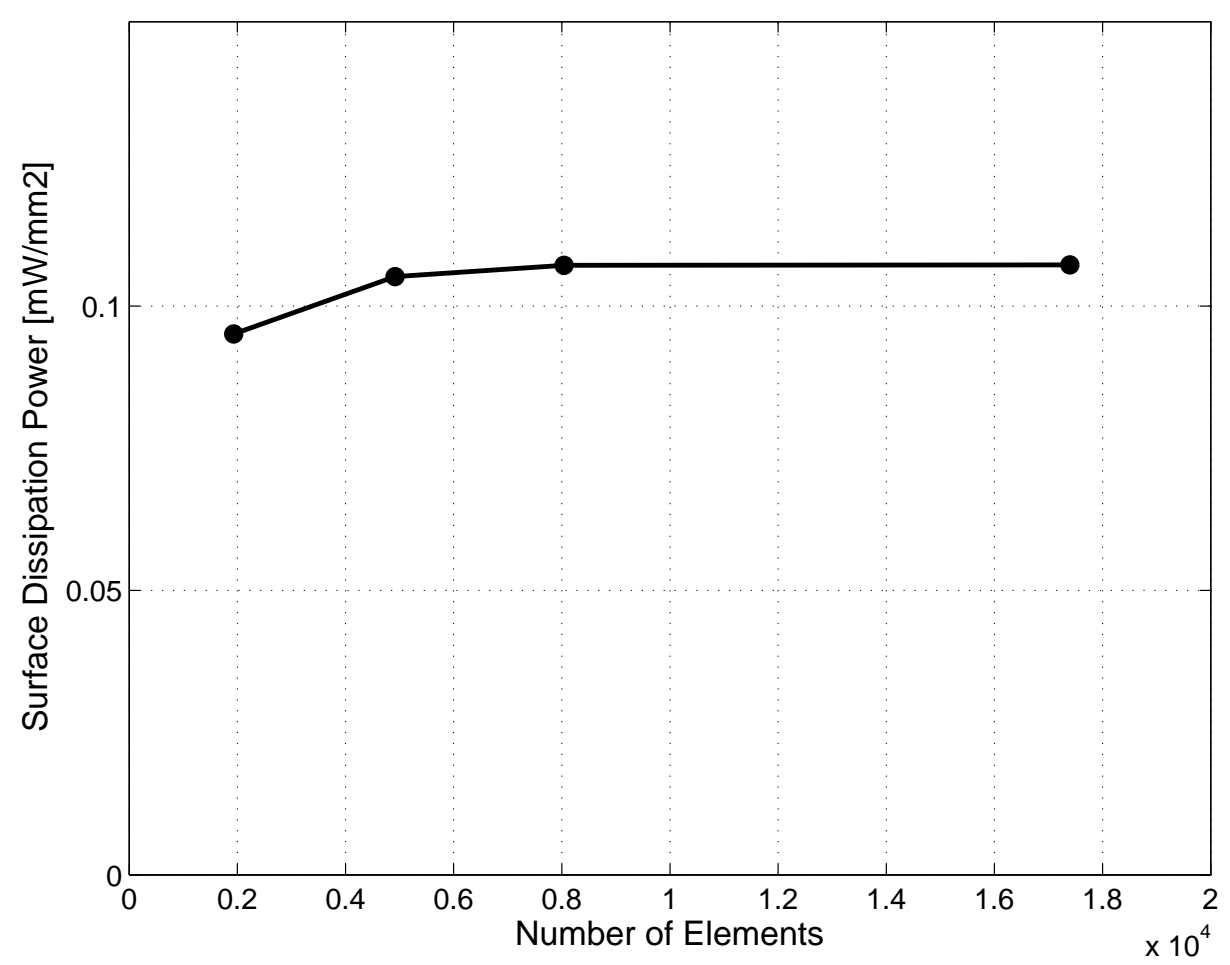

Figure 4.30: Surface Dissipation Power as a function of the number of elements in the mesh 


\section{Chapter 5}

\section{Volume Fraction Calculation}

\subsection{Introduction}

In this Chapter a tool that will help to define the volume fraction $V_{f}$ and the dimensions of the representative volume element (RVE) will be developed. The aim is to determine which is the typical volume fraction and RVE based in an digital image of the actuated polymer film (APF) obtained with optical microscopy.

This will be done in a MATLAB ${ }^{\circledR}$ script where digital image processing, computational geometry and statistics will be used. MATLAB ${ }^{\circledR}$ Image Processing Toolbox will be used. The whole process will be explained in the next sections using an example.

\subsection{Program Structure}

\subsubsection{Image Processing}

The analysis begins with a color image obtained from an optical microscope (Leitz Laborlux $12 \mathrm{ME}$ ) of the APF. Figures 5.1 and 5.2, show two regions of an APF sample. As it can be seen in these pictures, the size distribution of the ellipsoids is not very uniform. Also, the size of the ellipses is smaller that what is expected to be used in the applications of the APF's. At the present time, the research group that has developed the APF is investigating for a method to control the size of the droplets that, after stretching, will morph to ellipsoids. It is expected that for the application the size of the actuators will be larger and more uniform. The aforementioned Figures will be used to explain the process proposed for the calculation of the volume fraction.

The first goal is to convert Figures 5.1 and 5.2 into binary images. In order to do this the color images are first converted to gray scale. Then, adaptive histogram equalization (adapthisteq command in MATLAB ${ }^{\circledR}$ ) is applied to improve the image contrast. This type of equalization is called adaptive because instead of taking the image as a whole, it operates on different areas improving the contrast locally. This is useful if the image has non-uniform illumination [29]. After improving the contrast, the threshold that will define whether a pixel will be black or white is calculated using the graythresh command. Then, the gray scale image is converted to black and white employing the im2bw command and the 


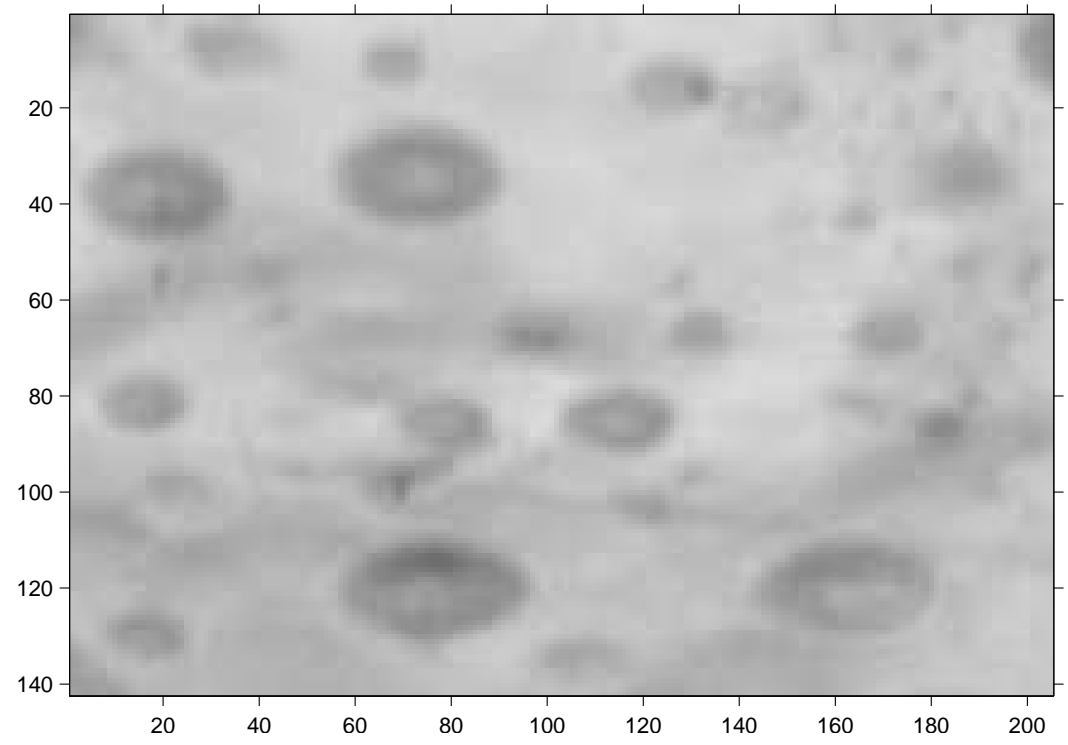

Figure 5.1: Image A of the actuators embedded in PVA (by Morris [41])

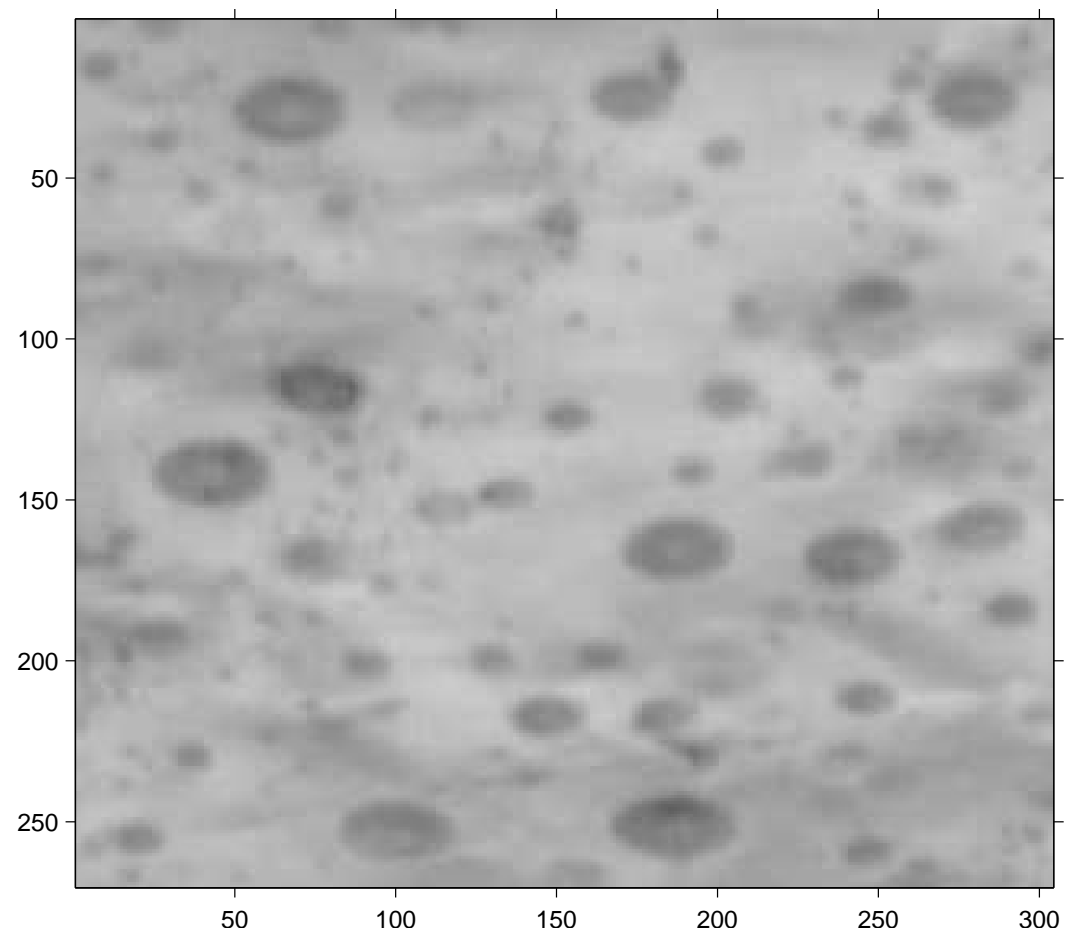

Figure 5.2: Image B of the actuators embedded in PVA (by Morris [41]) 


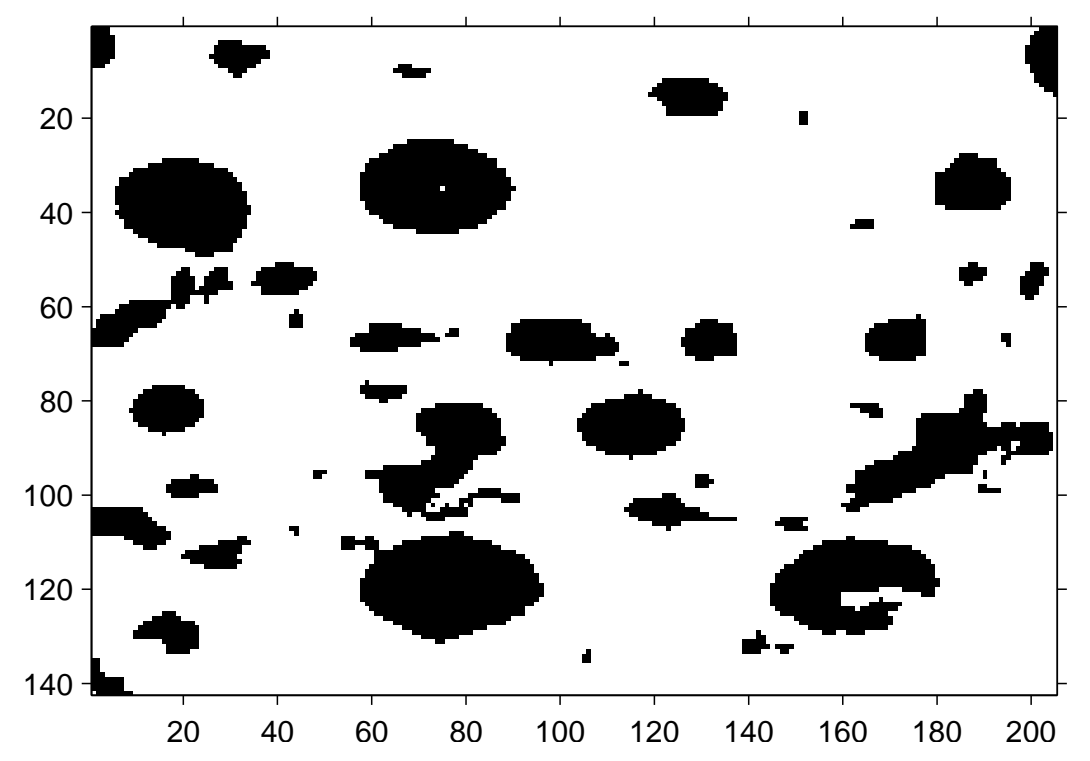

Figure 5.3: Image A converted to black and white

threshold calculated in the previous step.

At this point, the original images are now converted to a black and white images as it can be seen in Figures 5.3 and 5.4. In these Figures, it can be seen that there are some white spots in the interior of a few ellipses. Thus, the next step will be to fill the interior of the regions using the command imfill.

After filling the interior of the regions, the images are morphologically opened and closed using the commands imopen and imclose, respectively. The combination of these two operations will smoothen the contours of the regions and will break thin connections between neighboring regions [29].

As it can be seen in Figures 5.5 and 5.6, the boundaries are smoother, the interior regions are full and some of small regions were eliminated.

At this stage, all the modifications to the original images are done. The next step is to detect all the connected regions in the binary images, using MATLAB ${ }^{\circledR}$ 's command bwconncomp. Then the command regionprops is used to analyze the properties of all the regions found. In this script, the area, minor and major axes, eccentricity, orientation, centroid and bounding box for each region are calculated. A detailed list of all the possible properties to be analyzed can be found in MATLAB ${ }^{\circledR}$ Documentation [40].

A criteria is set to discard all the regions that should not be considered as actuators. This script will discard all the regions for which their areas are outside a specified range and all the regions for which the eccentricity is smaller than a specified value. The eccentricity is given by,

$$
e=\sqrt{1-\left(\frac{2 a}{2 c}\right)^{2}}
$$

where $2 c, 2 a$ are the major and minor axis of the region, respectively. In the examples, some small regions are discarded because their area is smaller that the minimum and/or 


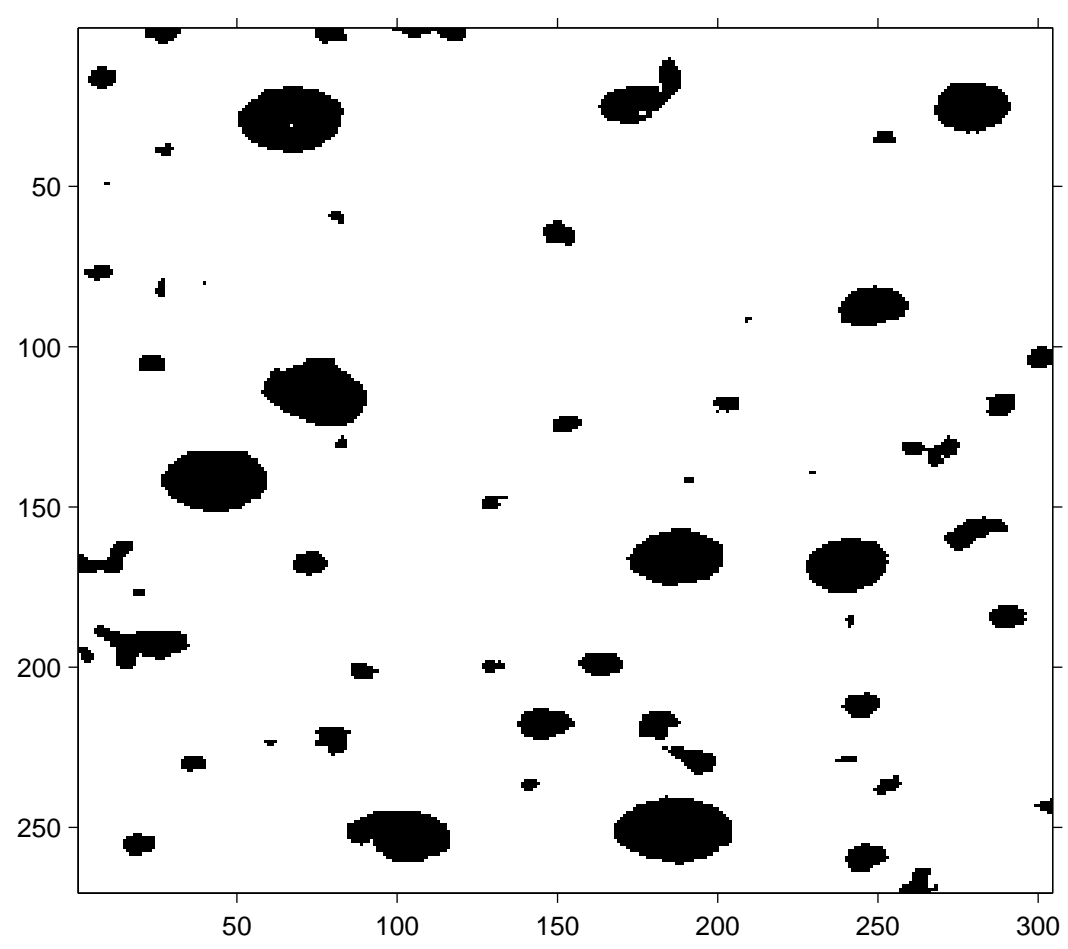

Figure 5.4: Image B converted to black and white

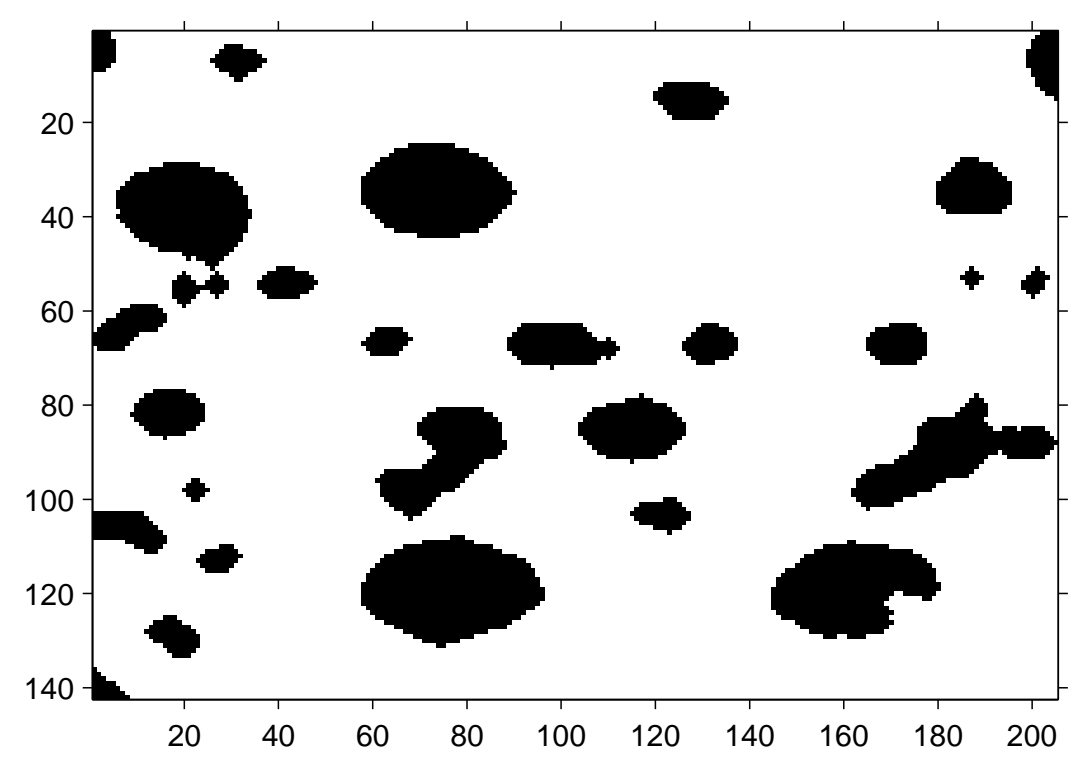

Figure 5.5: Image A after being filled and morphologically opened and closed 


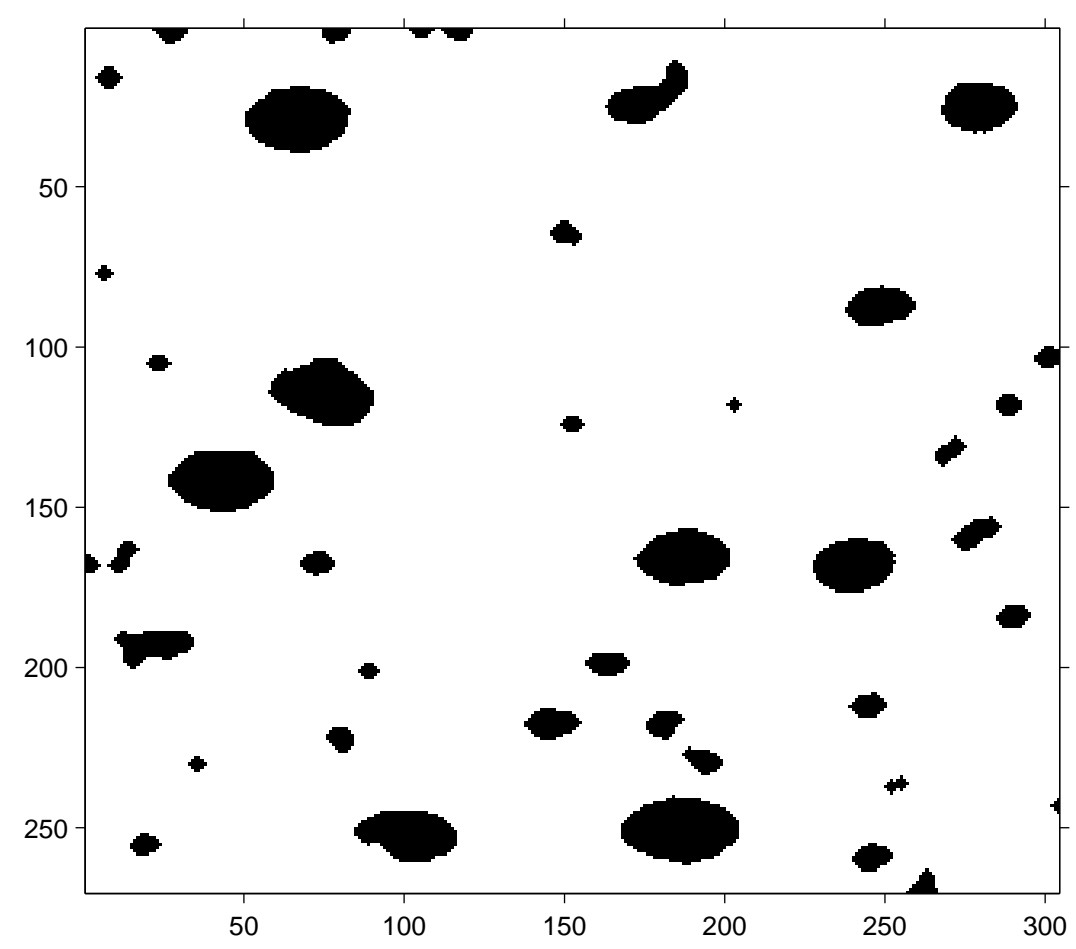

Figure 5.6: Image B after being filled and morphologically opened and closed

because their eccentricity is close to 1 . Obviously, the area and eccentricity criteria must be consistent with the image analyzed and it is likely that they will have to be modified to work correctly if the shape of the "typical" actuator changes.

After removing the regions that did not pass the filter, the resulting images are shown in Figures 5.7 and 5.8. At this point, all the image processing is done.

\subsubsection{Computational Geometry}

In this Section, the Voronoi diagram theory [9, §7] will be used to divide the image into cells. Each cell is associated with a site or point, that in this manuscript is the centroid of each actuator. The region defined by each Voronoi cell has the property of being the set of points that is closer to the Voronoi site associated with the cell than to any other Voronoi site. Figures 5.9 and 5.10 show the division of the images into the Voronoi cells associated with each actuator. In MATLAB ${ }^{\circledR}$, the functions voronoin and voronoi calculate the vertexes of the cells and plot the Voronoi diagram, respectively.

\subsubsection{Area Fraction}

The area fraction for each cell can be easily calculated, as the ratio between the area of the actuator and the area of the respective Voronoi cell. In the present work, the area fraction will be calculated only for the interior cells because for the cells that are on the boundaries of the image, nothing can be told about how far is the closest actuator. This explains why is so important to have a picture big enough to show several actuators. 


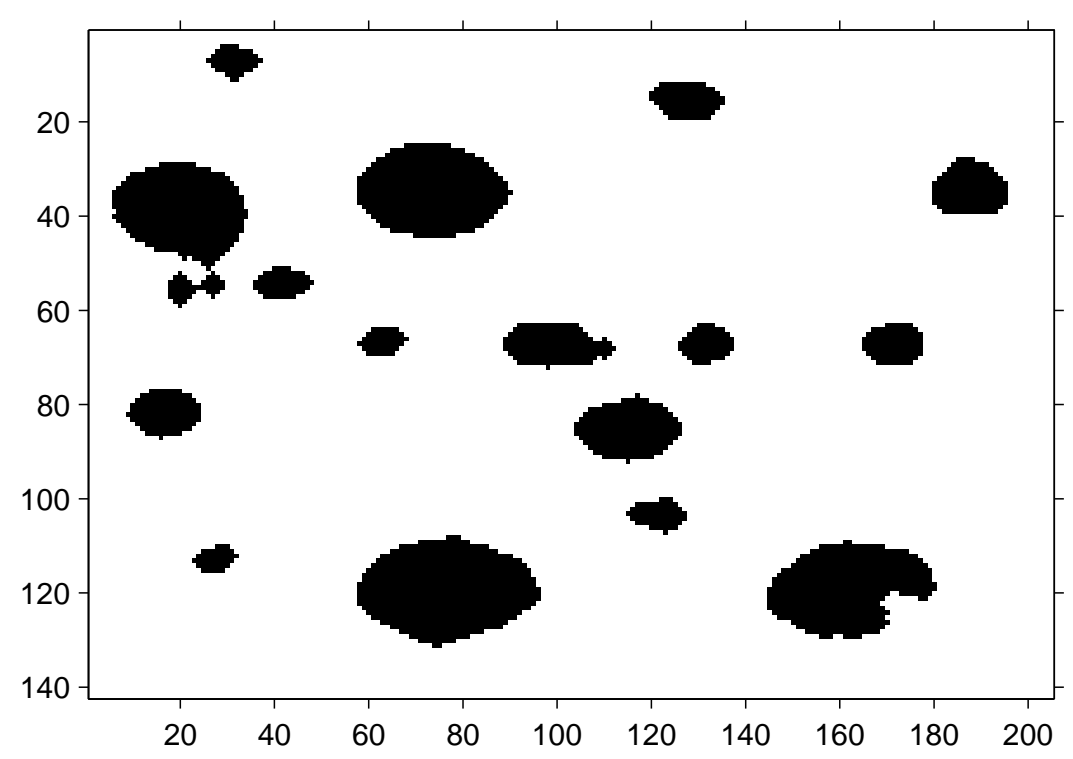

Figure 5.7: Image A after removal of the filtered regions

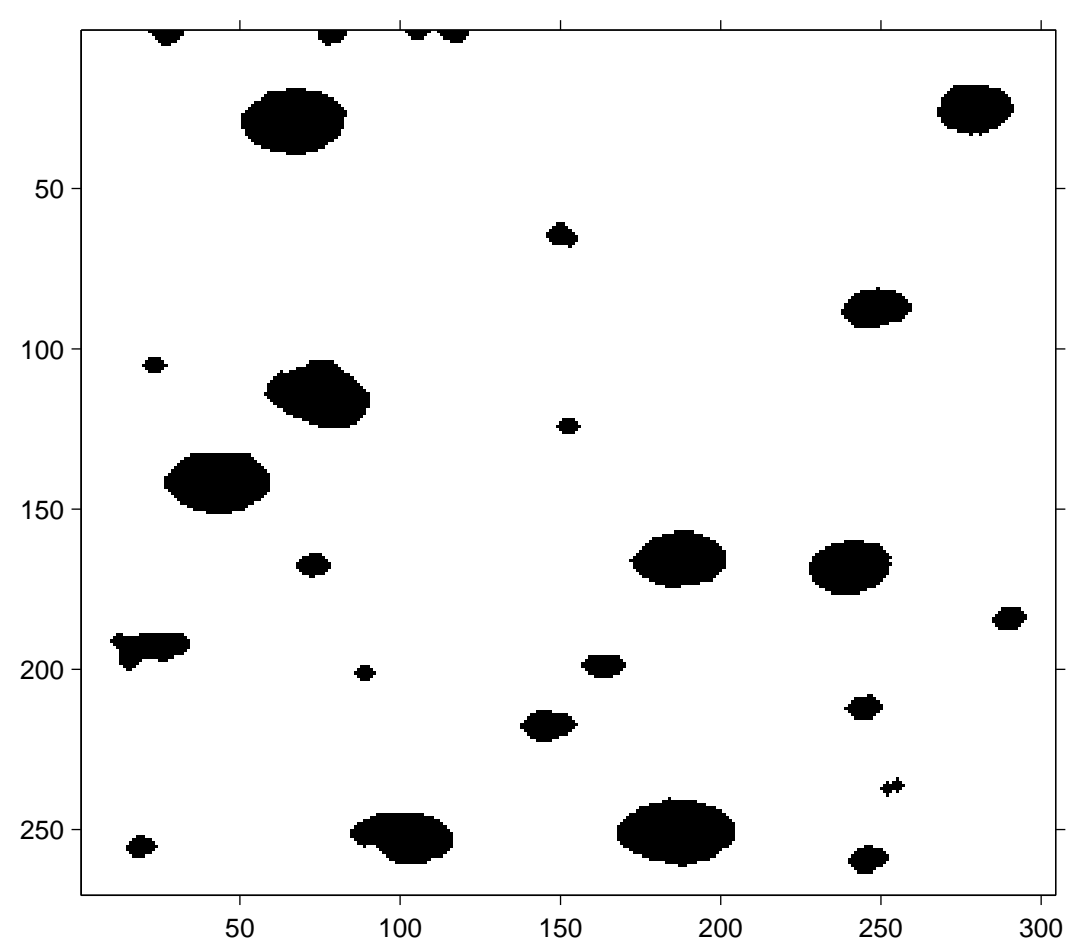

Figure 5.8: Image B after removal of the filtered regions 


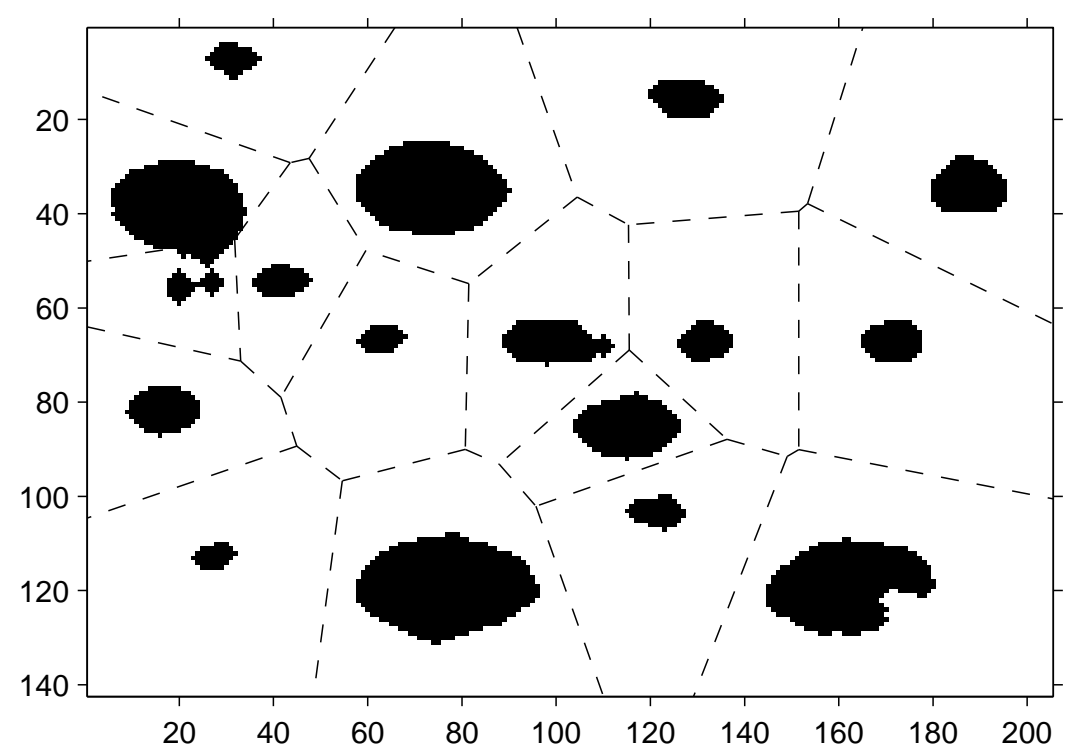

Figure 5.9: Image A - Actuators and their associated Voronoi cells

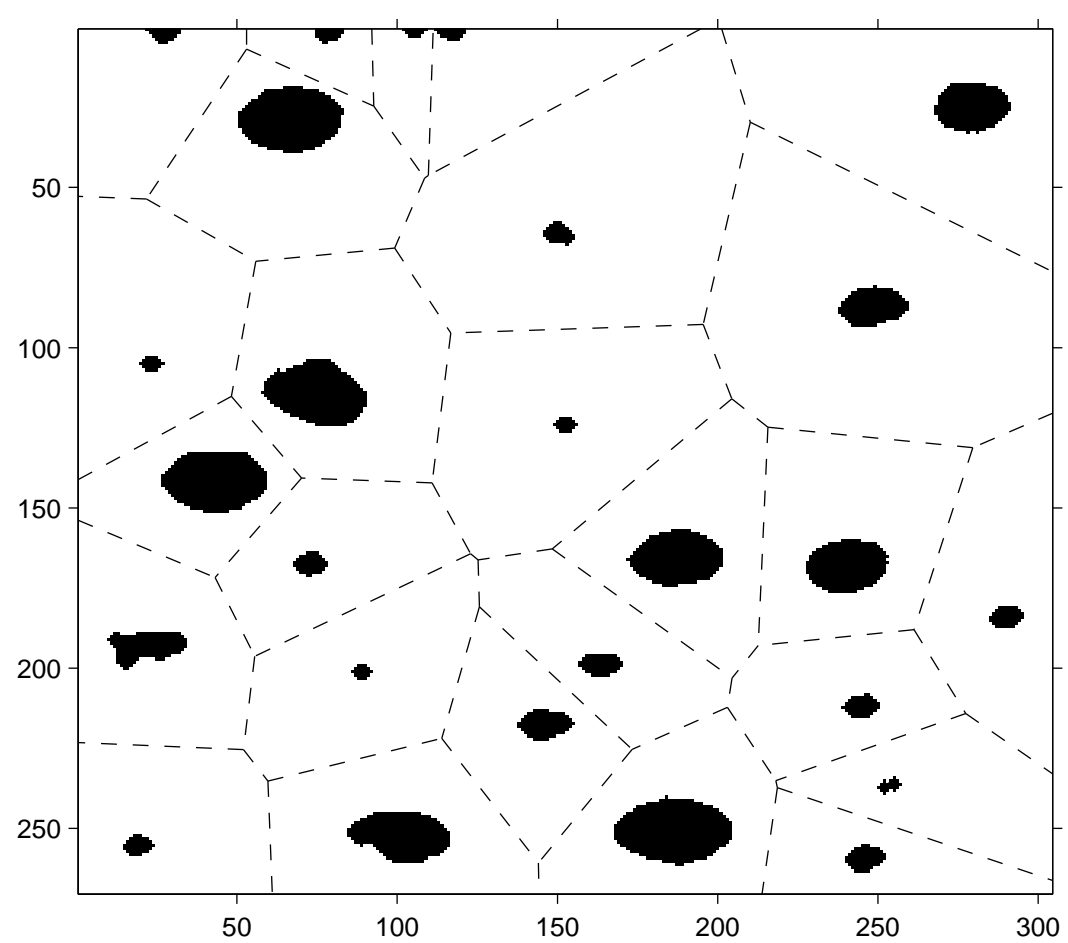

Figure 5.10: Image B - Actuators and their associated Voronoi cells 


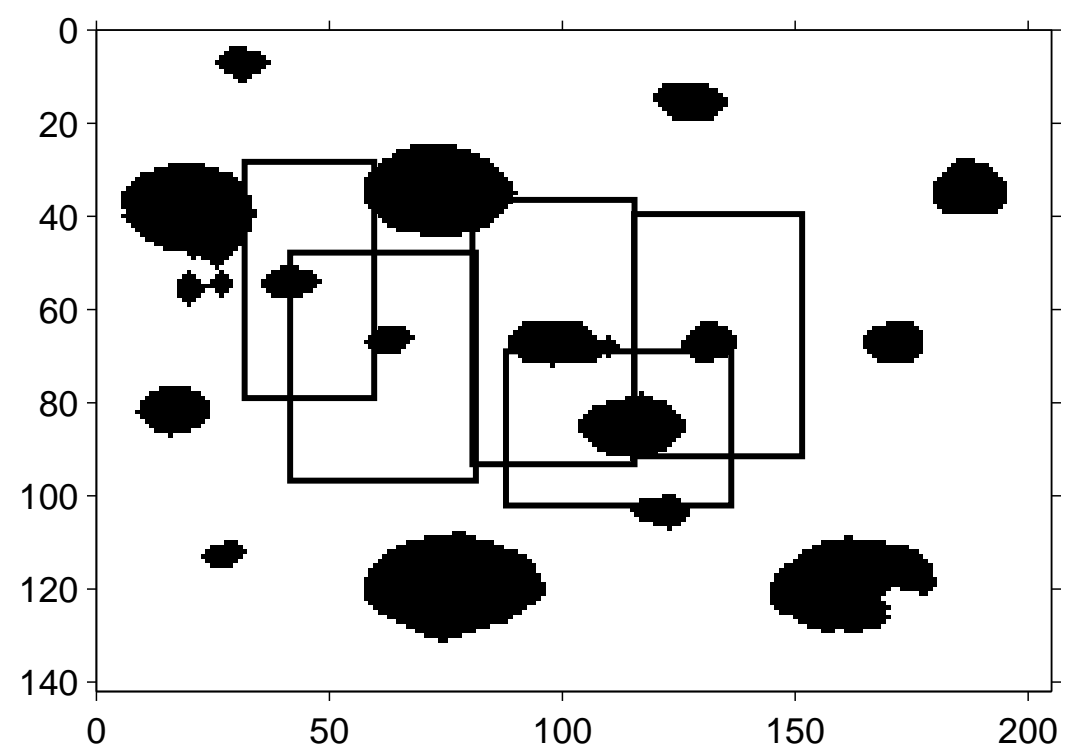

Figure 5.11: Image A - Bounding boxes for the interior Voronoi cells

\subsubsection{Volume Fraction}

From a two-dimensional image it is not possible to calculate the volume fraction $V_{f}$ without additional information. In order to estimate the volume fraction, the thickness of the film must be given and assumed to be constant. Furthermore, a three-dimensional geometry of the actuator must be assumed. For these examples, the three-dimensional geometry is considered to be a prolate spheroid and the major and minor radii for this geometry are assumed to be one half of the major and minor axes of the connected region (which were obtained from the binary images in Section 5.2.1).

\subsubsection{RVE dimensions}

In this Section, an approximated method to calculate the "typical" width and depth of the representative volume element (RVE) will be developed. These dimensions, and the thickness of the APF, are needed to analyze the APF using the finite element method and periodic boundary conditions, as it was done in Section 4. Based on the vertexes of the polygon of each Voronoi cell, an upper bound on the dimensions of the RVE can taken as the bounding box that encloses all the vertexes for each cell.

For the examples that are being used in this Chapter, the bounding boxes for the interior cells are displayed in Figures 5.11 and 5.12. Here, the boxes are assumed to be aligned with the $x-y$ directions. Hence, it is expected that the $x$ direction in the original image is parallel to the direction in which the APF was stretched.

As it was mentioned before, the dimensions of the RVE calculated here, constitute an upper bound. As it can be seen in Figures 5.11 and 5.12, there is some overlap between the bounding boxes, so the dimensions of the true RVE are smaller.

A lower bound on the RVE dimensions could be built considering the largest rectangle inscribed inside each cell. Although this problem has known solutions [2, 43], it is far from 


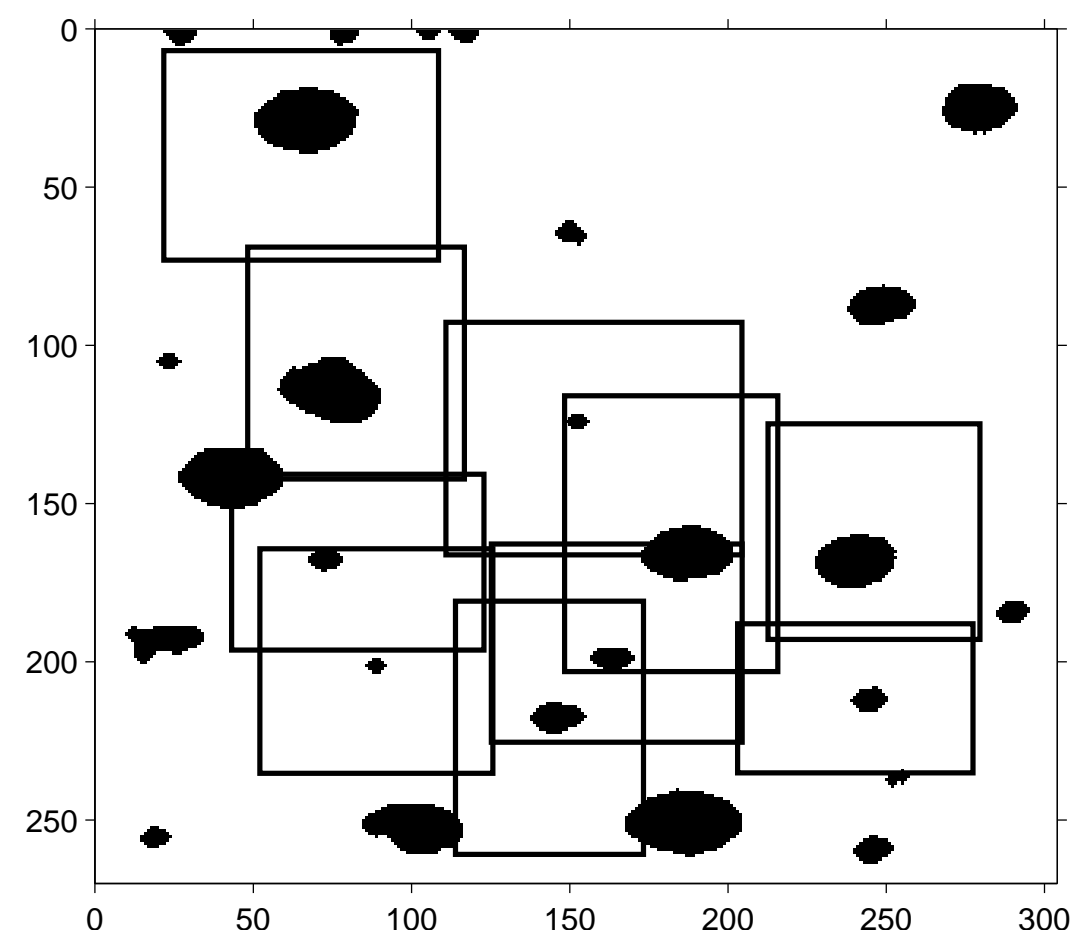

Figure 5.12: Image B - Bounding boxes for the interior Voronoi cells

\begin{tabular}{|cc|cc|}
\hline & & $\bar{x}$ & $s$ \\
\hline Actuator Area & {$\left[\mathrm{\mu m}^{2}\right]$} & 30.1664 & 31.4807 \\
Area Fraction & {[]} & 0.0632 & 0.0523 \\
Volume Fraction & {[]} & 0.0191 & 0.0209 \\
RVE Width & {$[\mu \mathrm{m}]$} & 74.9373 & 10.1906 \\
RVE Depth & {$[\mu \mathrm{m}]$} & 68.4344 & 11.5257 \\
\hline
\end{tabular}

Table 5.1: Results for the mean value $\bar{x}$ and standard deviation $s$ estimators

simple to implement, and therefore will not be included in the present work.

\subsection{Results}

The results for Image B (Figure 5.2) will be included in this Section. The source code of the complete program is included in Appendix E. The thickness of the APF sample depicted in Figures 5.1 and 5.2 is $36 \mu \mathrm{m}$ [41]. This thickness will be used in the calculation of the volume fraction of the present example.

The mean value and standard deviation estimations for different variables are listed in Table 5.1. The estimates on the area of the actuators are based on a sample size of 26 (all the actuators in the picture), while the volume fraction, area fraction and RVE dimensions are based on a sample size of 10 (only the interior cells). 


\section{Chapter 6}

\section{Conclusions and Future Work}

\subsection{Future Work}

\subsubsection{Temperature Dependence}

In the present work it was assumed that the process is isothermal. If desired, the mechanical properties of the materials used in the model can be made temperature dependent. A thermal analysis should be done to evaluate the change of the temperature within the RVE due to the hysteresis cycles.

\subsubsection{Torque Measurement}

The torque generated by the actuators when they are subjected to an electric field must be assessed. Although an analytical expression has been proposed [22, 21, Equation 1], this equation can not be immediately used because one of the parameters, the dielectric anisotropy $\Delta \epsilon$ is not known due to non-uniformly aligned regions of the liquid crystals in the actuators [21]. The determination of the torque generated by the actuators by indirect messurement will be necessary to input the magnitude $A$ in (4.2). Some of the possible techniques are discussed in this section.

\section{Optical Perfilometer}

Once the material science group develops the process to apply a conductive coating to the top of the film, the following experiment could be done. A sample of the APF is actuated, keeping the potential differential fixed. The surface of the APF is analyzed with an optical perfilometer, which requires no physical contact with the sample. When the displacements stabilize, the profile of surface is recorded. Then, the load in the FE model is changed until the magnitude of the displacements is similar to the experimental observation. This method is advantageous because it would involve measuring the response of the film as close as it would be in the final application of the APF. The major drawback is that the application of the conductive coating has not been successfully developed, yet. In addition, the College of Engineering and Mineral Resources at West Virginia University does not own this piece of equipment at the present time. 


\section{Fringe Pattern}

A sample of the APF is placed between two indium tin oxide (ITO) coated glasses, the potential differential is applied and depth of the valleys is measured using optical interferometry. Once the displacements are known, the applied torque in the FE model could be varied until a similar response is observed. If one of the surfaces is glued the glass, it might help to avoid interference between the fringe patterns created by the each tip. Using this technique, the measurement is possible but not very easy to do. This APF would lack of the conductive thin film, which modifies the mechanical behavior of the film. Furthermore, the APF used in this case would be different to the one that is intended for the application, for aerodynamic flow control for example, where the conductive coating is required.

\section{Micro Indenter - Force Control}

The bottom of a medium sized sample (e.g. $25 \mathrm{~mm}$ by $25 \mathrm{~mm}$ ) of the APF is glued to an indium tin oxide (ITO) coated glass, and another ITO coated glass is laid on top of the film. The micro indenter tip is moved until it makes contact with the top glass. Then, the micro indenter is set to force control. The force is set to zero and the displacement of the top glass is recorded, while the voltage differential is applied to the coated glasses.

If the mechanical properties of the base film are known, a FE analysis can be done using the script listed in Appendix E.1 and the applied load can be back-calculated so that the displacement of the analytic rigid surface matches the experimental displacement of the top glass.

\section{Contact Perfilometer}

As for the method that employs an Optical Perfilometer, the procedure for the application of the conductive coating should the established, before the following experiment can be done. The electric potential is applied, and after waiting until the displacements stabilize (viscous effects), a replica of the surface is made. This can be done applying a release agent and casting an UV curable resin, for example, on the top surface of the deformed APF. The necessity of the replica is because the perfilometer sensor could be damaged if it gets in contact with the conductive coating, when the potential differential is applied. The surface of the replica can be later analyzed with a contact perfilometer. Once the displacements are known, the applied torque in the FE model could be varied until a similar response is observed.

\subsubsection{Base film mechanical properties}

For the calculation of the dissipation and response of the APF, the base film material mechanical properties should be determined for the selected or candidate materials. The parameters of the $\mathrm{BB}$ model have been calculated for some elastomers using experimental data from the literature [10] and the program developed in Chapter 3. The calculated parameters and the goodness of fit are listed in Table 3.1.

In order to fit the parameters of the BB Model the following tests are suggested by Bergstrom [10], 
- Uniaxial tensile testing at three different true strain rates [6].

- Uniaxial compressive testing at three different true strain rates [5].

To achieve a constant true strain rate, the testing machine must be set to displacement control and the cross-head displacement must follow an exponential law. The samples should be conditioned to remove the Mullins effect, introduced in Section 2.2.

Currently, the APF has the actuators embedded in polyvinyl alcohol (PVA). Cairns 20] indicates that it is unlikely to get reliable testing data for PVA because its mechanical properties are highly dependent on the degree of polymerization (DP) and the humidity of the sample. Furthermore, it is improbable that for most of the possible applications, where the APF would be exposed to the environment (e.g. aerodynamic flow control), the base film will be PVA due to its solubility in water [48.

\subsection{Conclusions}

In this research effort, a set of analytical and numerical tools for the design and analysis of actuated polymer films has been developed. It is important to understand the difference between analysis and design. At the design stage there are, usually, several unknowns in terms of the geometry, material properties and loads. Therefore, the tools used for design should be able to cope with many different cases with enough simplicity that allows to study a large number of combinations of the uncertain variables in a reasonable amount of time. Sometimes, some simplifications must be done, for the sake of speed. On the other hand, the analysis tools must be as accurate and precise as possible, because in the analysis stage a great part of the original unknowns that were present at the design stage have been already quantified and the number of possible combinations has been narrowed.

Sophisticated three-dimensional models like the ones presented in Section 4.3 .4 give the most accurate and precise results, while simplified two-dimensional models, like the ones presented in Section 4.3.6, allowed to run a large number of cases (about twenty-five in this manuscript) in an affordable amount of time. Although there are still many unknowns of the actuated polymer films, in the present work the three-dimensional models have been used to define a ratio between the results of these and a two-dimensional model. This ratio can be used as a design tool, to extrapolate the results of the simplified two-dimensional models to the three-dimensional case, as a function of the volume fraction.

A remarkable fact from the results of the three-dimensional models (Table 4.5), is that as the volume fraction $V_{f}$ decreases, both the total dissipation power and the roughness reach threshold values. For the dissipation power, it can be clearly seen in Figure 4.13 that the dissipation should be close to $2.5 \times 10^{-5} \mathrm{~mW}$, if the volume fraction is extrapolated to zero. Conversely, the surface dissipation power, tends to zero as the volume fraction tends to zero (Figure 4.12). The latter conclusion is physically sound, because if a single actuator, which has a finite dissipation power, is embedded in a infinite medium, the dissipation power per unit surface should be, in the limit, equal to zero.

A similar behavior can be observed in Figure 4.14, for the roughness and surface dissi-

pation ratios as a function of the volume fraction. While the roughness ratio has a plateau 
for volume fractions smaller than $1.5 \%$, the ratio for the surface dissipation power is smaller than 0.1 for $V_{f}=0.7 \%$.

Several interesting observations can be extracted from the parametric analyses done in Section 4.3.6. It is worth to mention that while the roughness decreases with increasing excitation frequencies, the surface dissipation power follows the opposite conduct (Figures 4.15 and 4.16, respectively). This behavior arises from the viscous network of the base film material model. For a creeping material it is expected that the strains will grow with time, if the load is kept along the time. In the case of the actuated polymer films, the higher the frequency, the less time that the base film will have to deform. Thus, the roughness is reduced at higher frequencies. Regarding the surface dissipation power, higher frequencies of excitation will cause more displacement reversal cycles in the base film. From basic mechanics, it is known that the dissipation in a dash-pot or damper is associated to the first derivative of the displacement with respect to time, which for a sinusoidal movement becomes maximum when the displacement is reversed from positive to negative or vice versa.

The roughness and the surface dissipation power were higher, when the applied torque was increased (see Figures 4.17 and 4.18). It is important to notice that in order to increase the roughness by a $78 \%$, the dissipation is triplicated. This fact puts in evidence the nonlinear behavior of the viscous effects in the analyzed models.

When the vertical position of the actuator was changed, by translating it closer to the top surface, both the dissipation and the roughness increased. The opposite response was obtained when the actuator was moved closer to the bottom surface.

Another geometric parameter that was varied to study its effect in the results, is the aspect ratio of the actuator. Although only three different aspect ratios were investigated, the roughness and the surface dissipation power seem to follow a decaying exponential law, as it is shown in Figures 4.21 and 4.22 .

From the mesh dependency analysis, it can be concluded that for a two-dimensional model with an RVE width of $120 \mu \mathrm{m}$ and thickness of $30.03 \mu \mathrm{m}$, the change in the results is marginal for meshes with more than 4900 elements (e.g. less than $0.1 \%$ change of the roughness). Also, the use of quadratic elements showed a very small variation in the results (about $0.4 \%$ roughness difference).

Large changes in the top film and actuator modulus did not show a significant impact in the results (Figures 4.25 to 4.28). Therefore, it could be said that the response of the APF model has a very small sensitivity to these parameters.

A parametric study of the base film material properties was not done because the material was purposely chosen to be very compliant. Furthermore, the base film material is characterized by several parameters and in contrast to geometric parameters, the material parameters can not be tailored as desired. In this work, the parameters for the hyperelastic behavior were fit from experimental data available from the literature for Hytrel 4056. The parameters that characterize the viscous behavior were chosen to be similar to the ones of Nitrile Rubber (Section 3).

For the dissipation calculation, a custom script was written in Python. Appendix B showed that ABAQUS ${ }^{\circledR}$ built in variable for this purpose yields an incorrect result, by estimating about twice of the true dissipation. The proposed method was compared with experimental data from Bergstrom [16], showing very good agreement.

As it can be seen in Figure B.1, the solution obtained from the Finite Element model is 
very close to the experimental results. This result supports the fact that the FE solution using the Bergstrom-Boyce material model is representative of the real behavior of elastomeric materials under cyclic loading.

For all the models analyzed in Chapter 4, the material properties for the base film were obtained from the literature for a chosen material candidate. But it is important to bear in mind that a procedure to fit any desired material, from experimental data, was developed in Chapter 3. The performance of this procedure was assessed by fitting several materials from experimental data available in the literature and calculating the goodness of fit.

Lastly, a novel approach was used in Chapter 5 for the determination of the volume fraction from digital images of the APF. The proposed technique also allowed to calculate the geometry of the "typical" RVE, which is very useful to analyze the APF using finite element analysis with periodic boundary conditions. For illustration purposes, an example was done using a digital image of an APF sample.

In conclusion, a varied set of powerful tools for the design and analysis of actuated polymer films were developed and implemented in this work. It is expected that these procedures will be of valuable help in the process of filling the gap from the laboratory, where the actuated polymer films are being developed, to their successful application. 


\section{Appendix A}

\section{Code of the program to fit the BB model}

\section{A.1 Main Program}

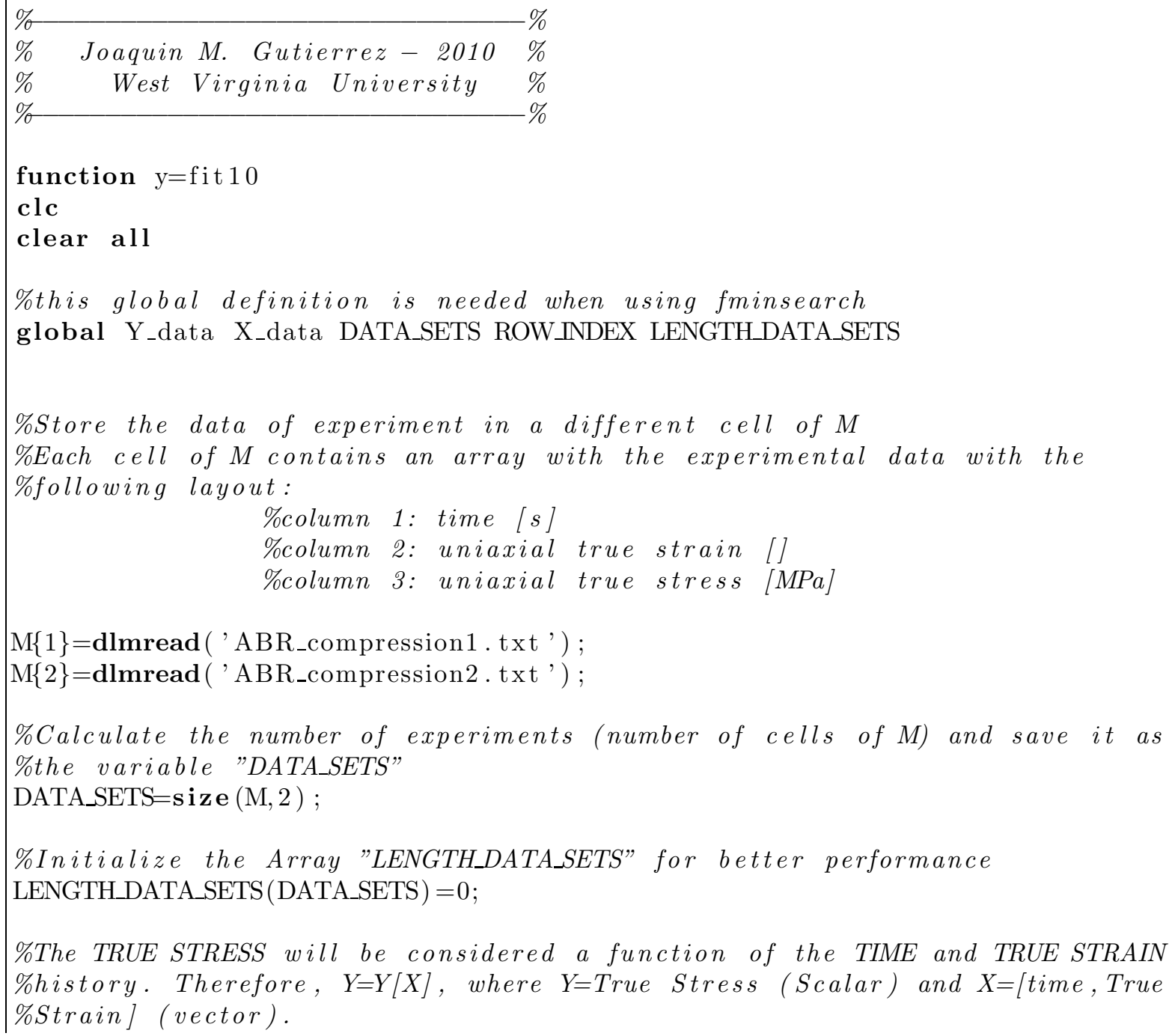




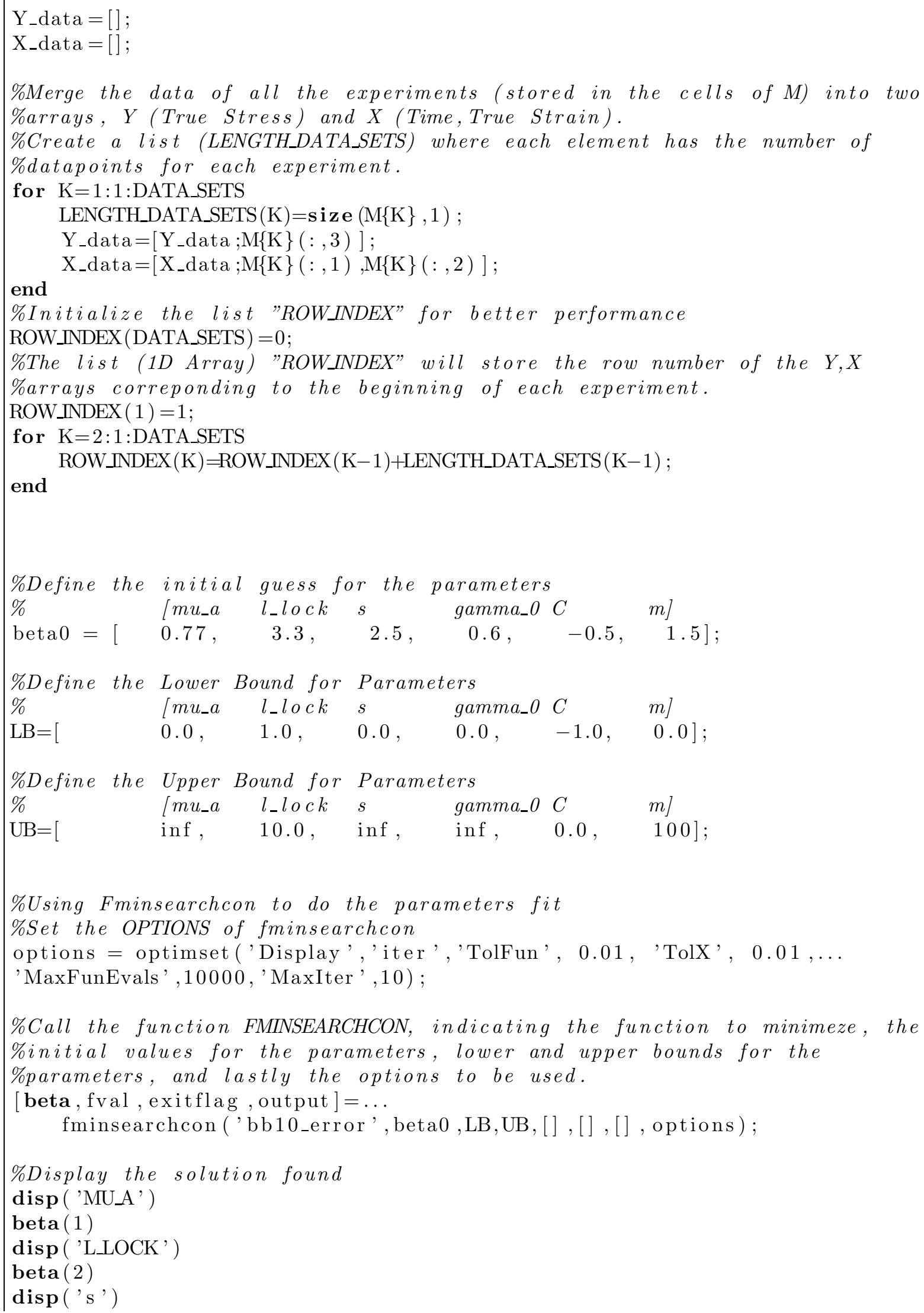




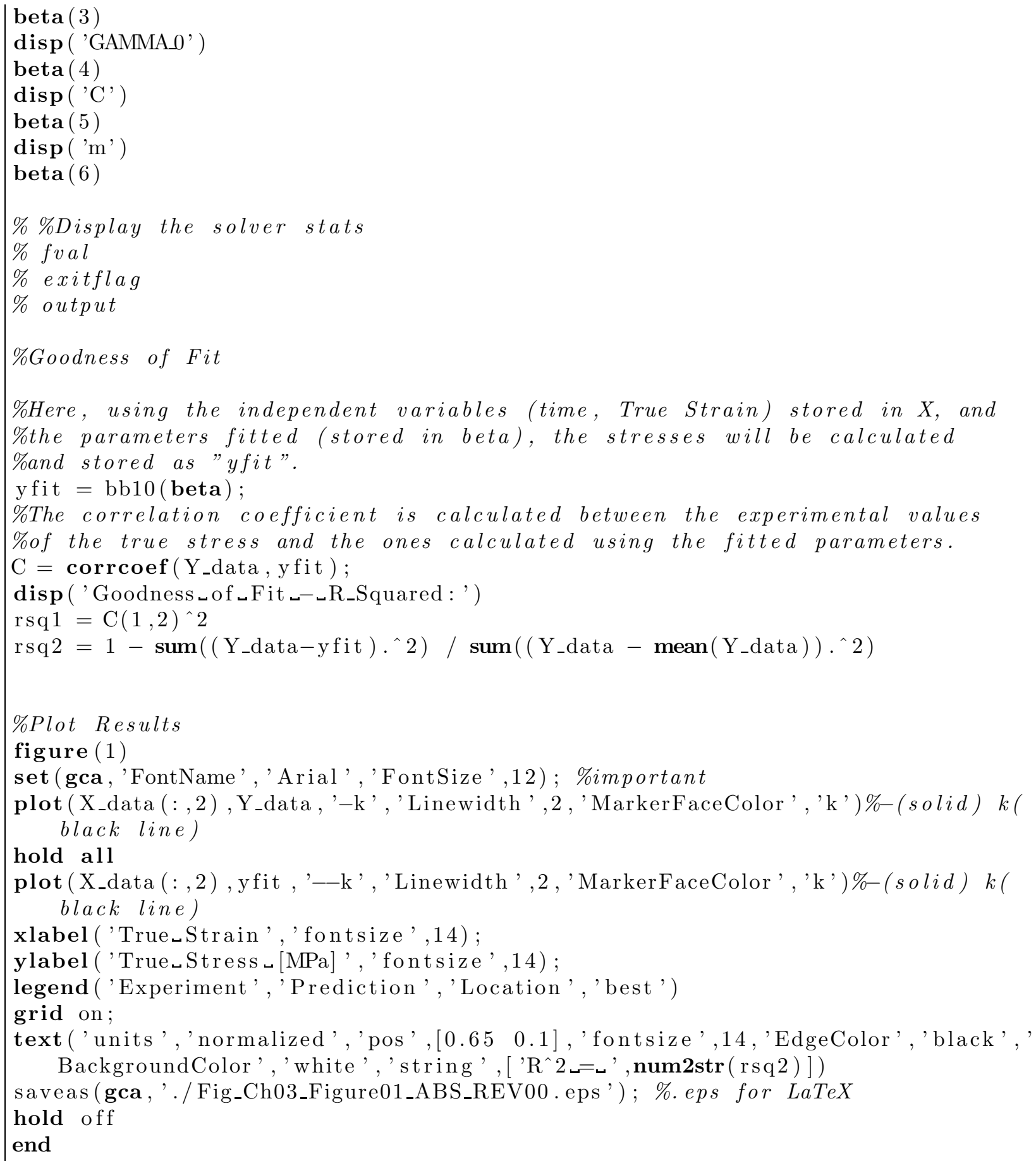

\section{A.2 Constitutive Equation Function}

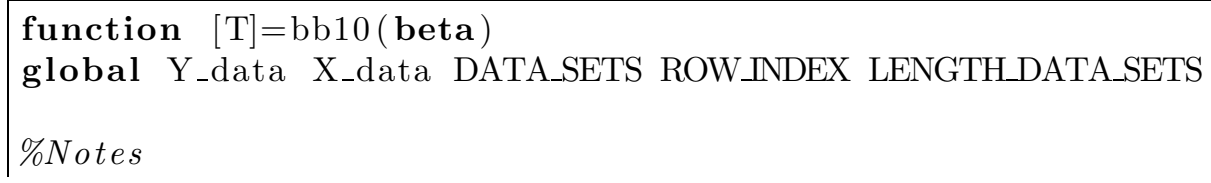




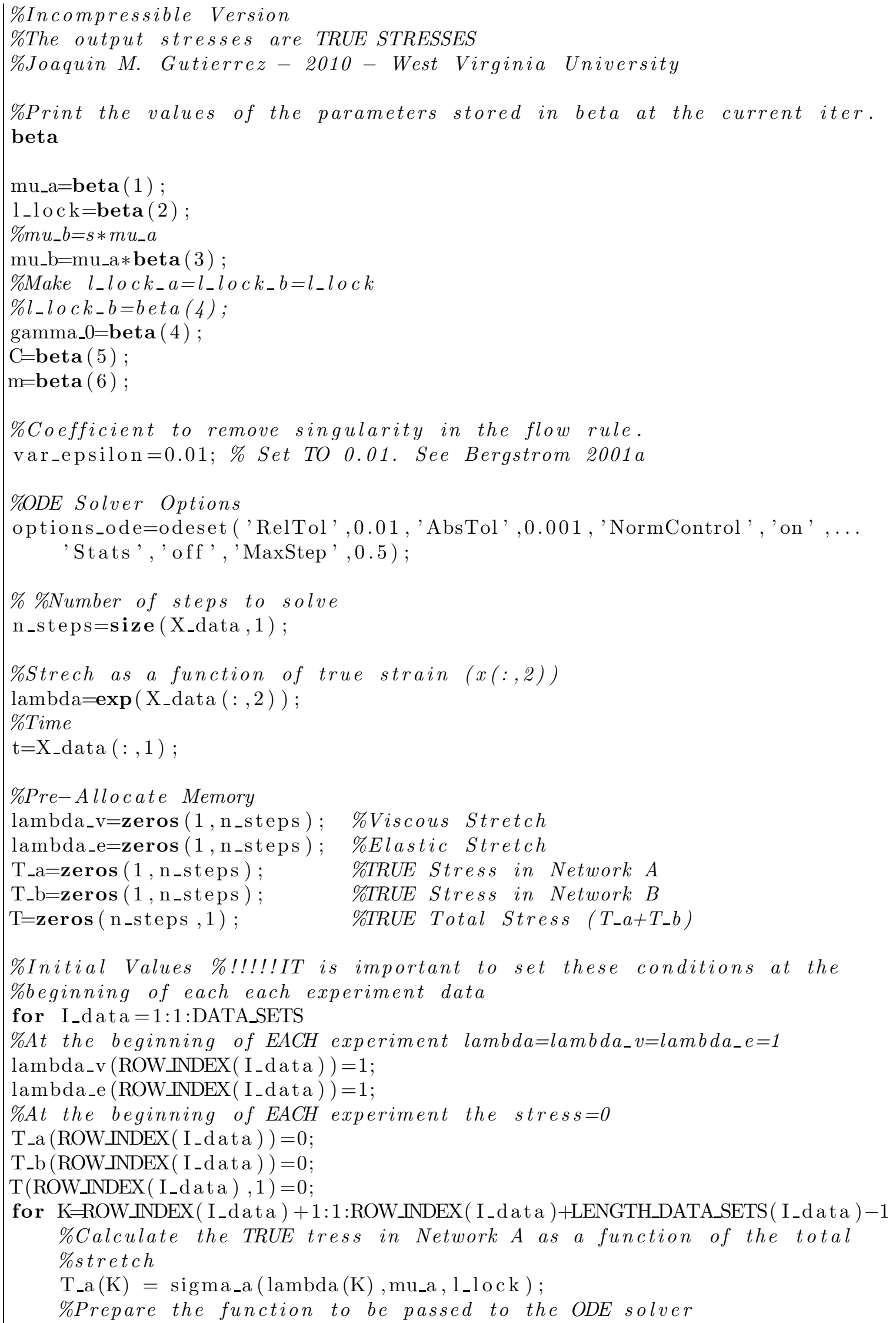




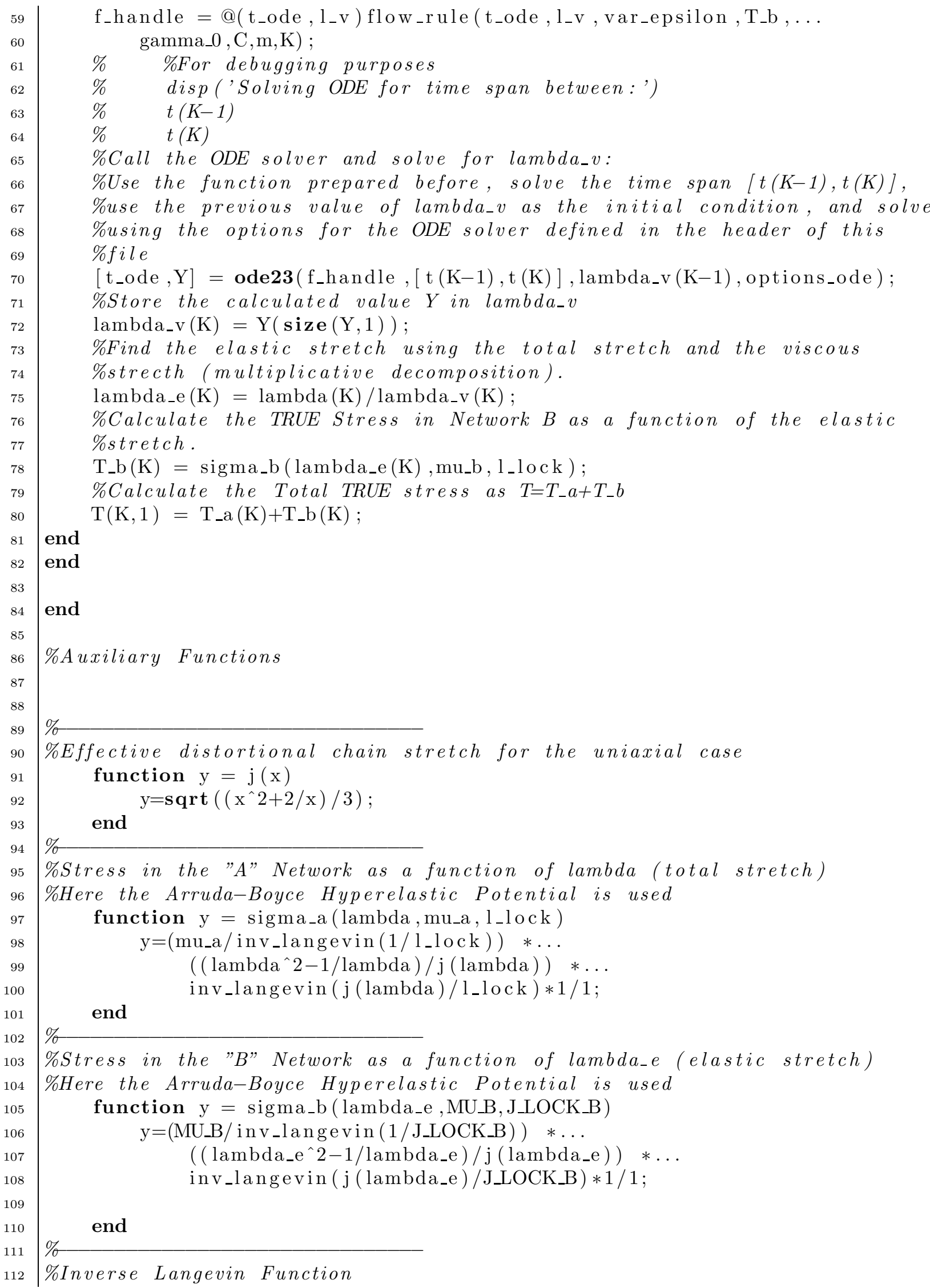




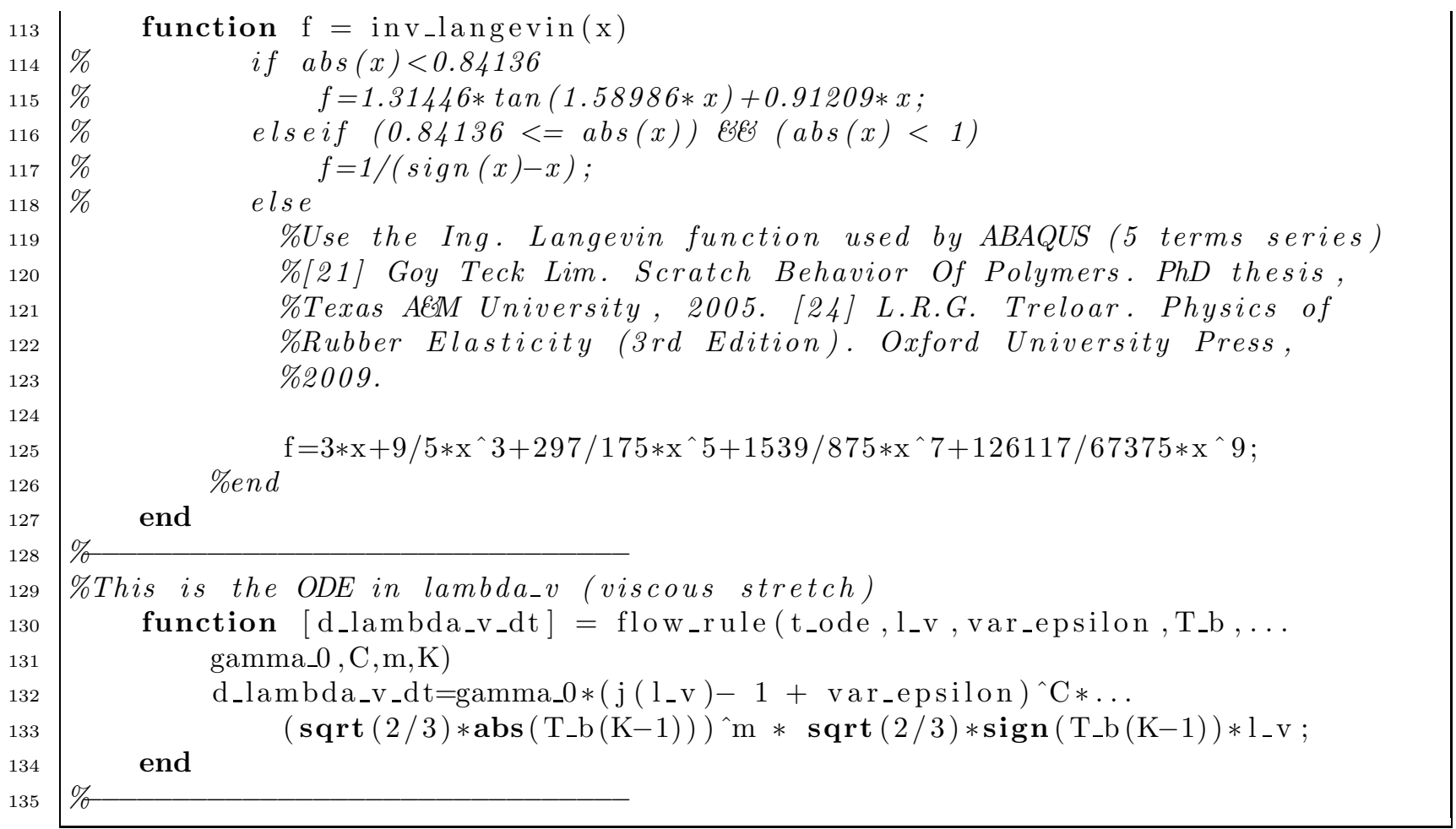

\section{A.3 Error Function}

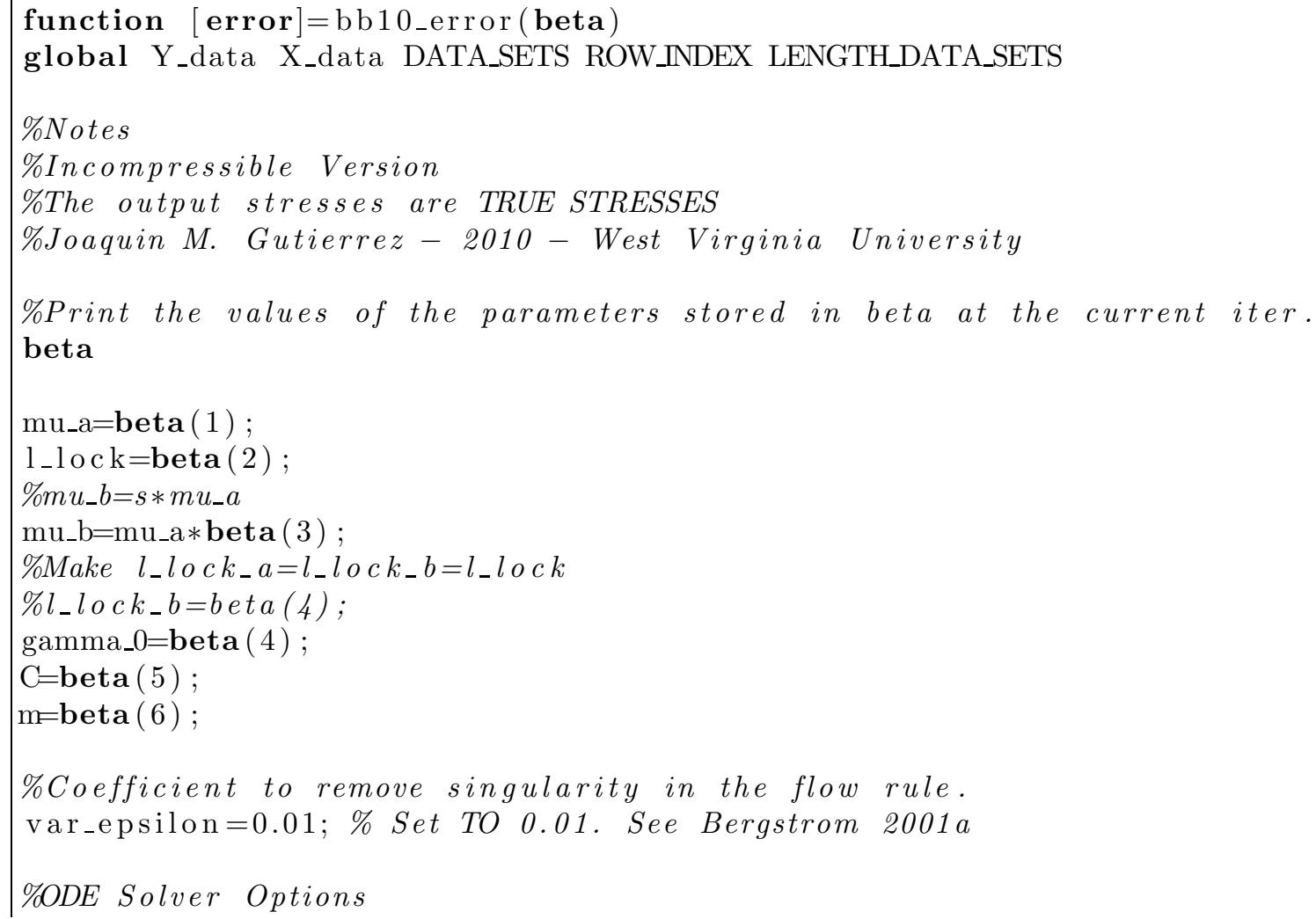




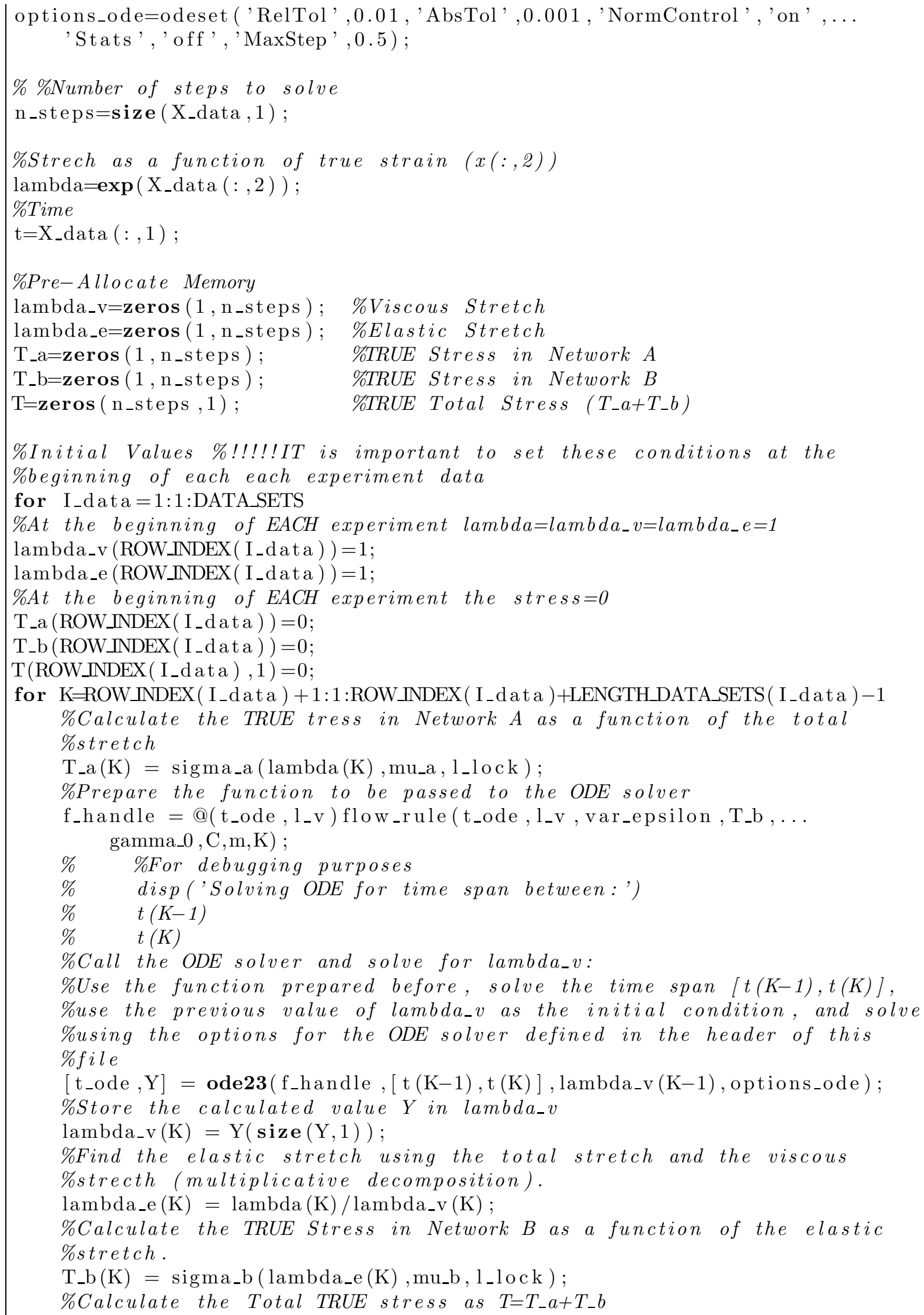


end

end

\%Calculate the error (difference) between the experimental data (Total True $\%$ Stress from Testing) and the predicted Total True Stress using the fitted \%parameters.

$\%$ Definition of the error,

\%OPTION "A": abs. val. of the difference between the prediction and the $\%$ measurement

\%error=norm $\left(Y_{-}\right.$data-T);

\%OPTION "B": MSD (Mean square deviation)

\%error $=100 * \operatorname{sum}\left(\left(Y_{-}\right.\right.$data $\left.\left.-T\right) \cdot{ }^{\wedge} 2\right) /$ length $(T)$;

\%OPTION "C": NMAD (Normalized Median Absolute Difference)

error $=100 * \operatorname{sum}\left(\operatorname{abs}\left(Y_{\text {_data }}-\mathrm{T}\right)\right) / \operatorname{sum}(\operatorname{abs}($ Y_data $))$;

end

\%Auxiliary Functions

$\%$

\%Effective distortional chain stretch for the uniaxial case

function $\mathrm{y}=\mathrm{j}(\mathrm{x})$

end

$y=\operatorname{sqrt}\left(\left(x^{\wedge} 2+2 / x\right) / 3\right) ;$

$\%$

\%Stress in the "A" Network as a function of lambda (total stretch)

\%Here the Arruda-Boyce Hyperelastic Potential is used

function $\mathrm{y}=$ sigma_a (lambda,mu_a, l_lock)

$\mathrm{y}=($ mu_a/inv_langevin $(1 /$ l_lock $)) * \ldots$

$(($ lambda^ $2-1 /$ lambda $) / \mathrm{j}($ lambda $)) * \ldots$

end

inv_langevin ( j (lambda)/l_lock $) * 1 / 1$;

$\%$

\%Stress in the "B" Network as a function of lambda-e (elastic stretch)

\%Here the Arruda-Boyce Hyperelastic Potential is used

function $\mathrm{y}=$ sigma_b (lambda_e, MU_B, J_LOCK_B)

$\mathrm{y}=($ MU_B/ inv_langevin (1/J_LOCK_B $)) * \ldots$

$(($ lambda_e^ $2-1 /$ lambda_e $) / j($ lambda_e $)) * \ldots$

inv_langevin ( j ( lambda_e ) J_LOCK_B $) * 1 / 1$;

end

$\%$

\%Inverse Langevin Function

function $\mathrm{f}=$ inv_langevin $(\mathrm{x})$

$\% \quad$ if abs $(x)<0.84136$

$\% \quad f=1.31446 * \tan (1.58986 * x)+0.91209 * x$;

$\% \quad$ elseif $(0.84136<=a b s(x)) 836(a b s(x)<1)$

$\% \quad f=1 /(\operatorname{sign}(x)-x)$;

$\% \quad$ else

\%Use the Ing. Langevin function used by ABAQUS (5 terms series) 


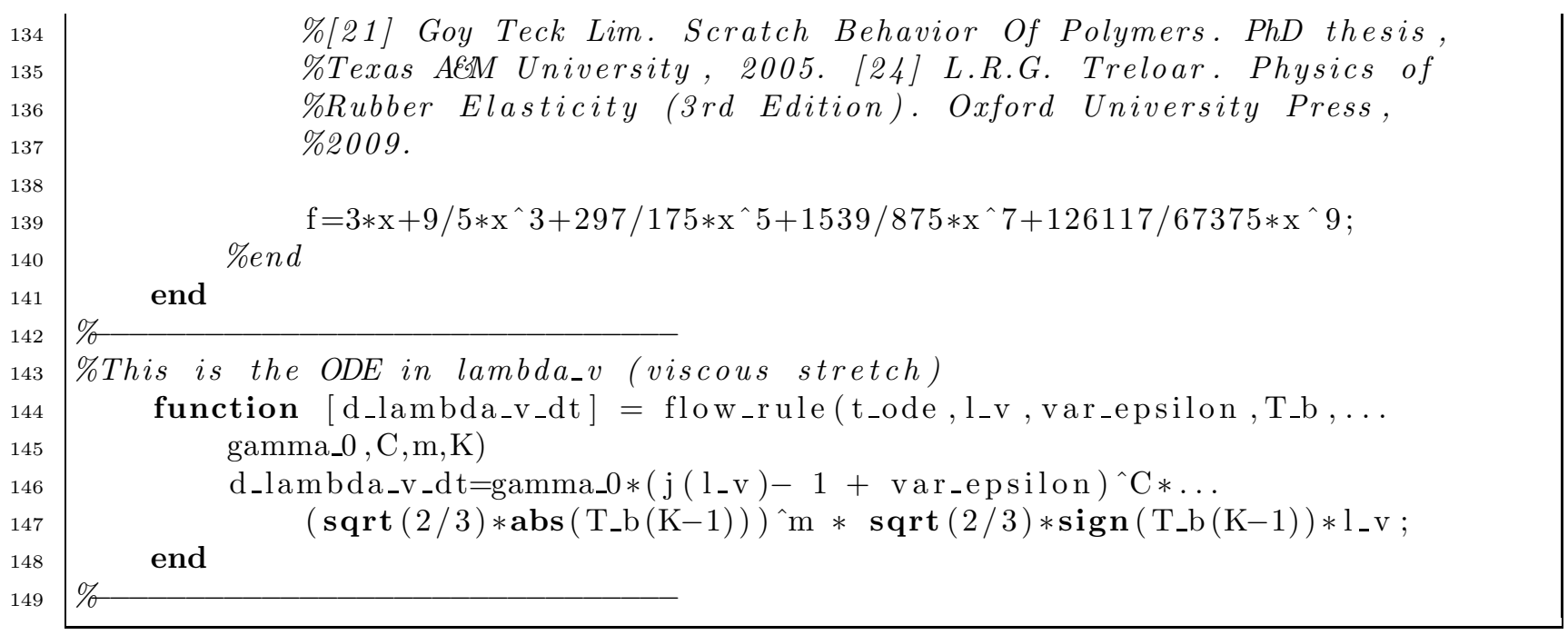




\section{Appendix B}

\section{Dissipation Calculation}

\section{B.1 Comparison}

The aim of this Section is to show that ABAQUS ${ }^{\circledR}$ built-in creep energy dissipation calculation (ALLCD) has a flaw, and therefore should not be used.

A simple uniaxial compression model will be simulated. The geometry, material properties, boundary conditions and loading are the same that were used in Reference [16, Figure $2]$.

The geometry is a disc with diameter $\phi=28 \mathrm{~mm}$ and height $h=13 \mathrm{~mm}$. The material properties used are $\mu_{A}=1.31 \mathrm{MPa}, \lambda_{A}^{\text {lock }}=\lambda_{B}^{\text {lock }}=3.00, d=2 / \kappa=0.004 / \mathrm{MPa}, s=$ $3.37, \dot{\gamma}_{0} / \tau_{\text {base }}^{m}=0.33 /\left(s \mathrm{MPa}^{m}\right), m=5.21$ and $c=-1.0$.

The bottom surface of the disk is restrained from moving in the vertical direction and the top surface will have an imposed displacement. In Reference [16], the loading is done by imposing a constant compressive true strain rate. In order to reproduce this in the present work, an amplitude defined by a table is used in the ABAQUS ${ }^{\circledR}$ model. Given the true strain rate $\dot{\epsilon}$ and the undeformed height of the sample $h$, the displacement of the top surface during the loading phase is described by,

$$
U_{2}(t)=-h(1-\exp (\dot{\epsilon} t))
$$

The maximum true strain to be achieved is -0.8 . Using a strain rate of $-0.05 / \mathrm{s}$, it takes $16 \mathrm{~s}$ to reach the maximum strain, and further $16 \mathrm{~s}$ to unload. The table used to define the displacement in ABAQUS ${ }^{\circledR}$ is listed in Table B.1.

Because this loading condition creates a uniform state of stress (the stress tensor is the same for all the points in the continuum), analyzing only one point is enough to understand the behavior of the whole. In Figure B.1 the true stress-strain 1 curve in the loading direction is plotted for one of the integration points in model (line with markers). These results are in very good agreement with the experimental results of Bergstrom and Boyce [16, Figure 2], which are also shown in Figure B.1.

The area enclosed by the experimental stress-strain curve at a strain rate equal to $0.05 / \mathrm{s}$ was calculated in AutoCAD, resulting in a dissipation energy density of $0.5878 \mathrm{MPa}$. The area enclosed by the stress-strain curve represents the dissipated energy density for the

\footnotetext{
${ }^{1}$ ABAQUS ${ }^{\circledR}$ refers to the true strain as logarithmic strain.
} 


\begin{tabular}{cc} 
Time $[\mathrm{s}]$ & $U_{2}[\mathrm{~mm}]$ \\
\hline 0 & 0.000 \\
1 & -0.634 \\
2 & -1.237 \\
3 & -1.811 \\
4 & -2.357 \\
5 & -2.876 \\
6 & -3.369 \\
7 & -3.839 \\
8 & -4.286 \\
9 & -4.711 \\
10 & -5.115 \\
11 & -5.500 \\
12 & -5.865 \\
13 & -6.213 \\
14 & -6.544 \\
15 & -6.859 \\
16 & -7.159 \\
17 & -6.859 \\
18 & -6.544 \\
19 & -6.213 \\
20 & -5.865 \\
21 & -5.500 \\
22 & -5.115 \\
23 & -4.711 \\
24 & -4.286 \\
25 & -3.839 \\
26 & -3.369 \\
27 & -2.876 \\
28 & -2.357 \\
29 & -1.811 \\
30 & -1.237 \\
31 & -0.634 \\
32 & 0.000 \\
& \\
\hline
\end{tabular}

Table B.1: Vertical displacement of the top surface as a function of time 


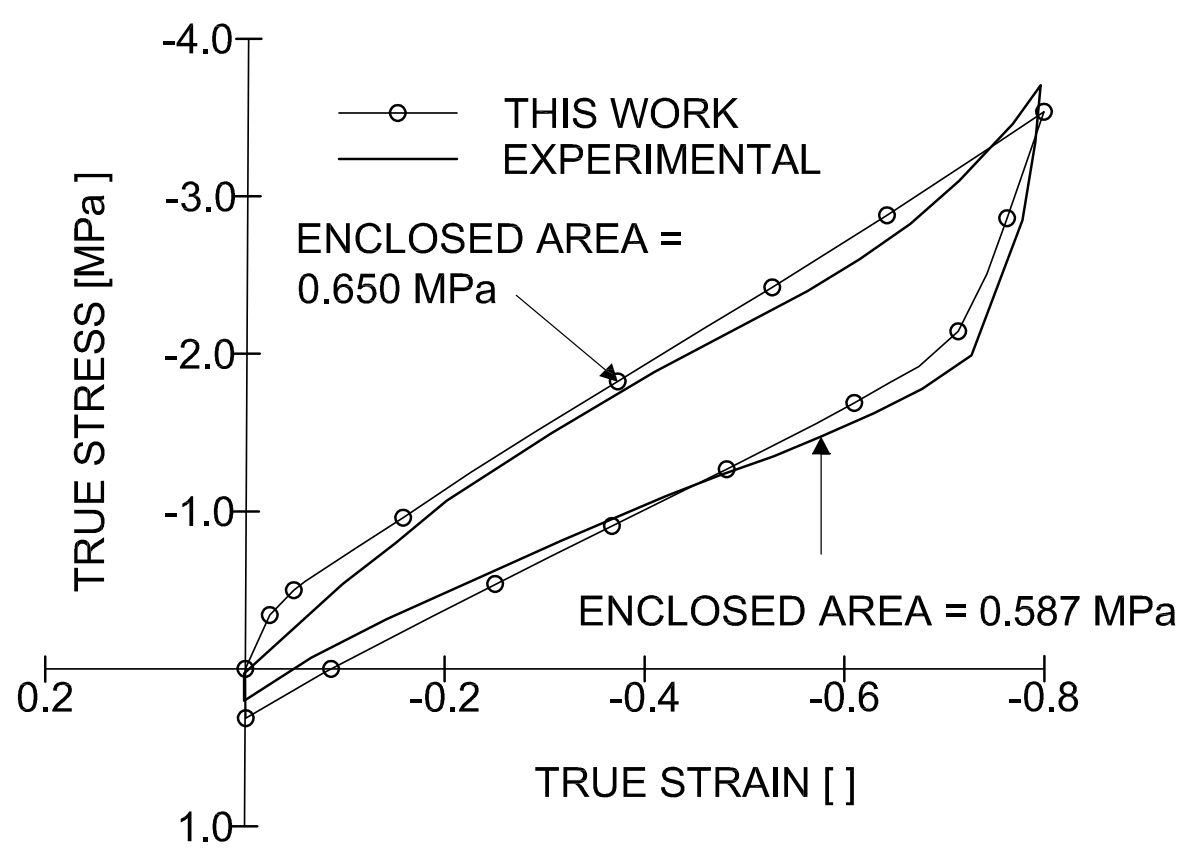

Figure B.1: Stress-Strain curve for strain rate 0.05 (Experimental data from [16, Fig. 2])

loading-unloading cycle due to the hysteretic behavior of the material. For the numerical example done in this work, the dissipated energy density $u$ is equal to $0.6501 \mathrm{MPa}$, which is in good agreement with the experimental results by Bergstrom and Boyce $(0.5878 \mathrm{MPa})$.

To calculate the energy dissipated in the whole model a simplification will be introduced, by multiplying the dissipated energy density by the undeformed volume of the element. Although this is not true because the Cauchy stress belongs to the deformed configuration, due to the low compressibility of the material used $(d=0.004 / \mathrm{MPa})$, the change in volume of the model is small. Therefore, the total energy dissipated in the sample used by Bergstrom and Boyce can be approximated by

$$
\mathscr{U}_{\text {creep }} \approx \pi \frac{\phi^{2}}{2} h u=8005 \mathrm{~mm}^{3} 0.5878 \mathrm{MPa}=4705 \mathrm{~mJ}
$$

On the other hand, when the total creep-dissipated energy is queried at the last time step through the history output variable ALLCD, the result given by ABAQUS ${ }^{\circledR}$ is $\left.A L L C D\right|_{t=32 \mathrm{~s}}=$ $10578.7 \mathrm{~mJ}$. The evolution of the variable ALLCD is included in Figure B.2.

The dissipation energy calculated by ABAQUS ${ }^{\circledR}$ is over twice as the energy calculated in (B.2) for the experimental sample. When the dissipation is calculated by numerical integration using the post-processing script developed by the author and presented in the following Section, the dissipated energy obtained is $5255.1 \mathrm{~mJ}$, which is in very good agreement with the experimental data. Thus, the dissipation calculation for the BB model can not be done 


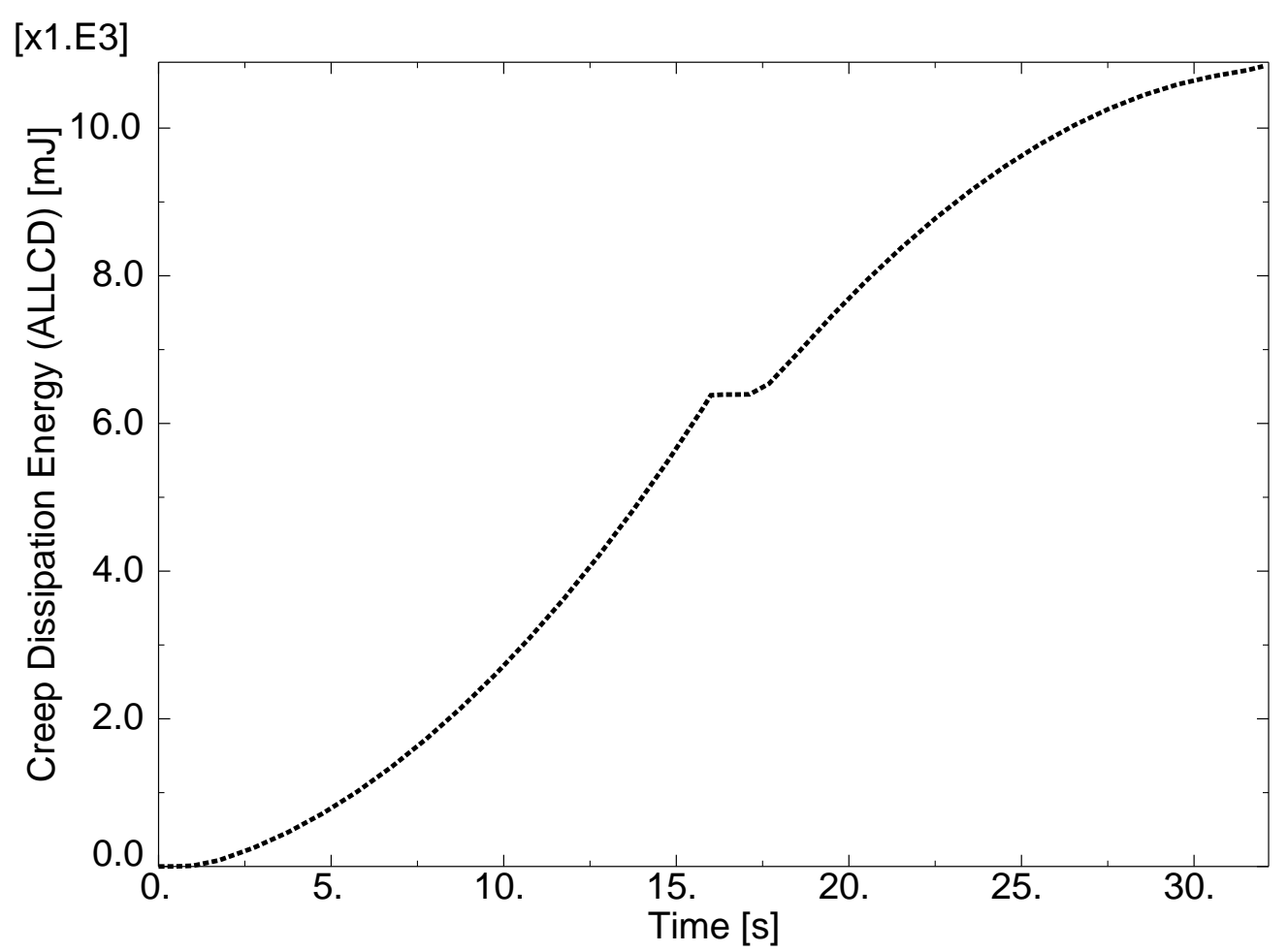

Figure B.2: Creep dissipation energy (ALLCD) for the whole model

using ABAQUS ${ }^{\circledR}$ built-in method and must be done using the script proposed by the author.

\section{B.2 PYTHON Script}

In order to calculate the dissipated energy correctly, a PYTHON script was developed. This script is ran after the analysis is done and it uses the output database from ABAQUS ${ }^{\circledR}$. The field outputs that this script must read are

LE Logarithmic Strain Tensor

S Cauchy Stress Tensor

IVOL Integration Point Volume

The script must be able also to query the history output ALLSE, the total recoverable (elastic) strain energy at the last frame (time step) of the analysis.

The total strain energy can be calculated using numerical integration as

$$
\mathscr{W}=\sum_{k=2}^{n} \sum_{l=1}^{m} \sum_{i=1}^{p}\left(\mathrm{LE}_{i}^{k, l}-\mathrm{LE}_{i}^{k-1, l}\right) \frac{\left(\mathrm{S}_{i}^{k, l}+\mathrm{S}_{i}^{k-1, l}\right)}{2} \frac{\left(\mathrm{IVOL}^{k, l}+\mathrm{IVOL}^{k-1, l}\right)}{2}
$$


where $n$ is the number of frames in the analysis (time steps), $m$ is the number of integration points in the model and $p$ is the number of independent components of the stress and strain tensors in the analysis. For example, in a three-dimensional analysis $p=6$, while in a plane strain analysis $p=3$. The first (outer) summation loops over the frames of the analysis, the second over integration points and the third over the components of the stress and strain tensors.

To save computational time, the integration can be carried out only in the regions made of hysteretic material. For an elastic material, the strain energy is equal to zero when the material is unloaded. Viscous materials exhibit a phenomena where the strains lag behind the stresses. This means that even when the stress is removed, the strain might not be equal to zero. Therefore, the script must subtract the recoverable elastic strain energy from the total strain energy in order to calculate the dissipated energy.

$$
\mathscr{U}_{\text {creep }}=\mathscr{W}-\left.\mathrm{ALLSE}\right|_{k=n}
$$

where $\mathscr{W}$ is given by $(\overline{B .3})$ and ALLSE $\left.\right|_{k=n}$ is the value of the recoverable strain energy at the last frame for the region where $\mathscr{W}$ was calculated.

A temporary variable is created to hold the frame repository. This noticeably speeds up the process. Further performance improvements could be done by accessing the output database using $\mathrm{C}++$ instead of PYTHON.

\section{B.3 Code Listing}

The following lines of code will be included in most of the scripts used to analyze the different models in Chapter 4. They are included here again, independently of the other code, for the sake of clarity.

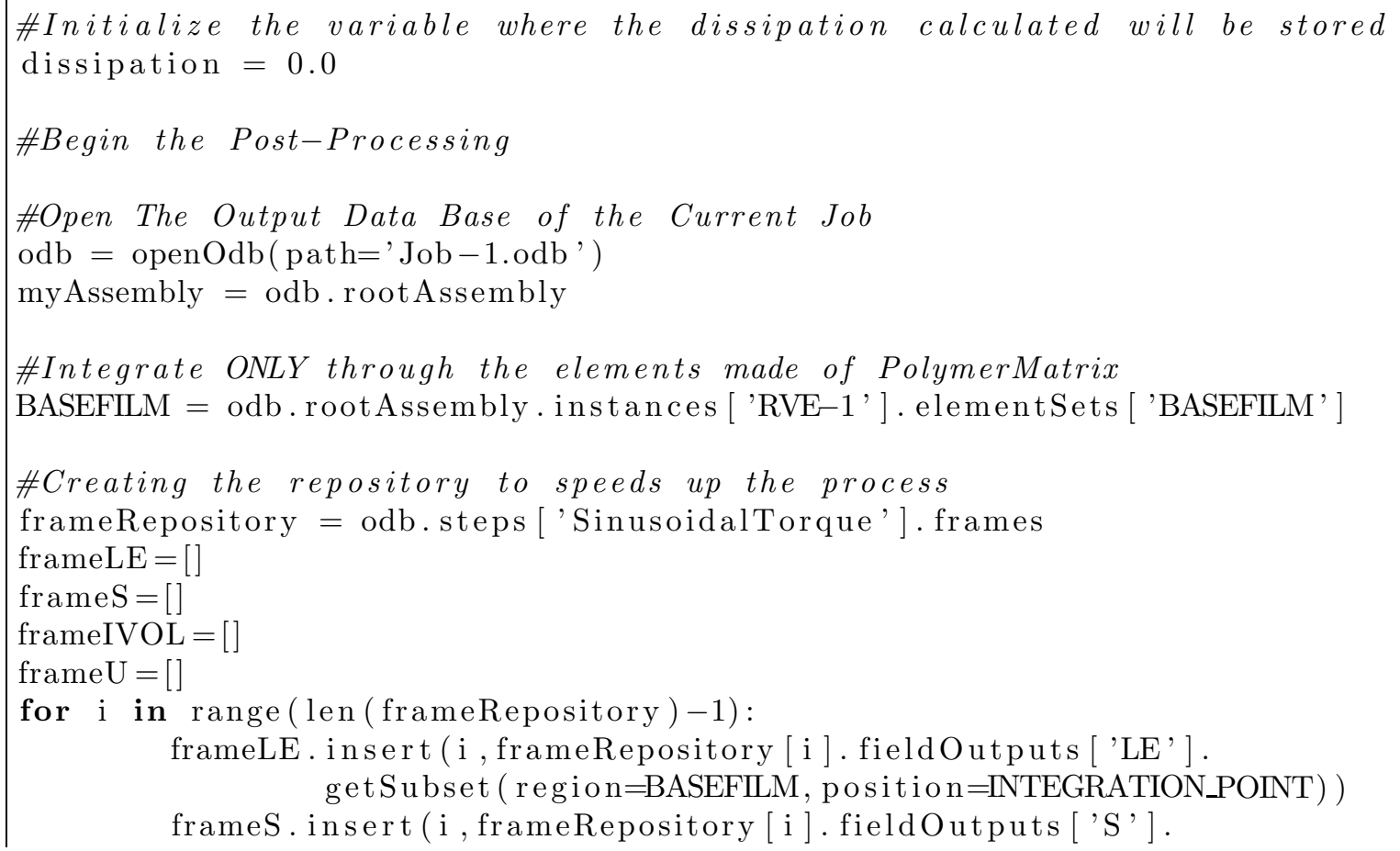


get $\mathrm{Subset}$ ( $\mathrm{r}$ e g i o n=BASEFILM, p o sitio $\mathrm{n}=$ INTEGRATION_POINT) $)$ frameIVOL . insert (i frameRepository [i ]. fieldOutputs ['IVOL'] . get $\mathrm{Subset}$ ( r e gi o n=BASEFILM, p o sitio $\mathrm{n}=$ INTEGRATION_POINT) $)$ frameU . insert (i, frameRepository [i ]. fieldOutputs ['U'] . get Subset ( region=TOP, position $=$ NODAL) )

\#Number of frames in the step to be integrated

N_frames=len (frameLE)

\#Initialize the following temporary variable

dummy $=0$

\#Do the integration. The index II loops over the integration points, JJ loops over the frames, KK over the different components of the stress/strain tensors.

for II in range( 0 , len (frameLE [0]. values)):

for JJ in range $\left(0+1, N_{-}\right.$frames $)$:

$\mathrm{x}_{-} \mathrm{c}=$ frameLE $[\mathrm{JJ}]$. values $[\mathrm{II}]$. data

$\mathrm{X}_{-} \mathrm{w}=$ frameLE $[\mathrm{JJ}-1]$. values [II ]. data

$y_{-} c=$ frameS $[\mathrm{JJ}]$. values $[\mathrm{II}]$. data

$\mathrm{y}_{-} \mathrm{w}=$ frameS $[\mathrm{JJ}-1]$. values [II ]. data

$\mathrm{v}_{-} \mathrm{C}=$ frameIVOL $[\mathrm{JJ}]$. values [II ]. data

$\mathrm{v}-\mathrm{W}=$ frameIVOL $[\mathrm{JJ}-1]$. values $[\mathrm{II}]$. data

dummy $=0.25 *\left(\mathrm{x}_{-} \mathrm{c}-\mathrm{x}_{-} \mathrm{w}\right) *\left(\mathrm{y}_{-} \mathrm{c}+\mathrm{y}_{-} \mathrm{w}\right) *\left(\mathrm{v}_{-} \mathrm{c}+\mathrm{v}_{-} \mathrm{w}\right)$

dummy_ $2=0$

for KK in range (0, len (dummy)):

dummy_2=dummy_2+dummy $[\mathrm{KK}]$

dissipation=dissipation+dummy_2

\#Subtract the Recoverable elastic strain in the THE BASE FILM (Polymer Matrix Set)

dissipation=dissipation -odb.steps ['SinusoidalTorque'] . historyRegions ['

ElementSet „BASEFILM'] . historyOutputs ['ALLSE']. data [-1][1]

print dissipation 


\section{Appendix $\mathrm{C}$}

\section{Relationship between the two- and three-dimensional loads}

Cairns et al. suggest that the actuators generate torque by volume [21, 22]. In the following Sections, the relationship between the surface traction that must be applied to generate torque by volume for the two- and three-dimensional cases will be developed.

\section{C.1 Three-dimensional case}

In the three-dimensional case the geometry of the actuator is a prolate spheroid, a particular case of an ellipsoid. The equation that describes the boundary of a prolate spheroid is given by,

$$
\frac{x^{2}+y^{2}}{a^{2}}+\frac{z^{2}}{c^{2}}=1, \quad c>a
$$

where $a, c$ are the minor and major radii, respectively.

The torque generated by a uniform traction acting in the surface of the actuator in the $z$ direction, can be calculated considering only $1 / 8$ of the actuator due to symmetry conditions.

If the differential torque is generated by a traction $F_{z}$ acting on the surface of the ellipsoid, times the arm, the differential torque is proportional to the volume. The arm is given by the height $y$ from the $x z$ plane to the surface where the traction is applied, as it can be seen in Figure C.1.

From (C.1), for the first octant, the distance $y$ to the $x z$ plane can be written as,

$$
y(x, z)=\sqrt{\left(1-\frac{z^{2}}{c^{2}}\right) a^{2}-x^{2}}
$$

Therefore, for every point in the $x z$ plane, the corresponding arm is given by (C.2). The torque generated by the actuator can be calculated as

$$
\text { Torque }=8 \iint_{\Omega} y(x, z) F_{z} d x d z
$$



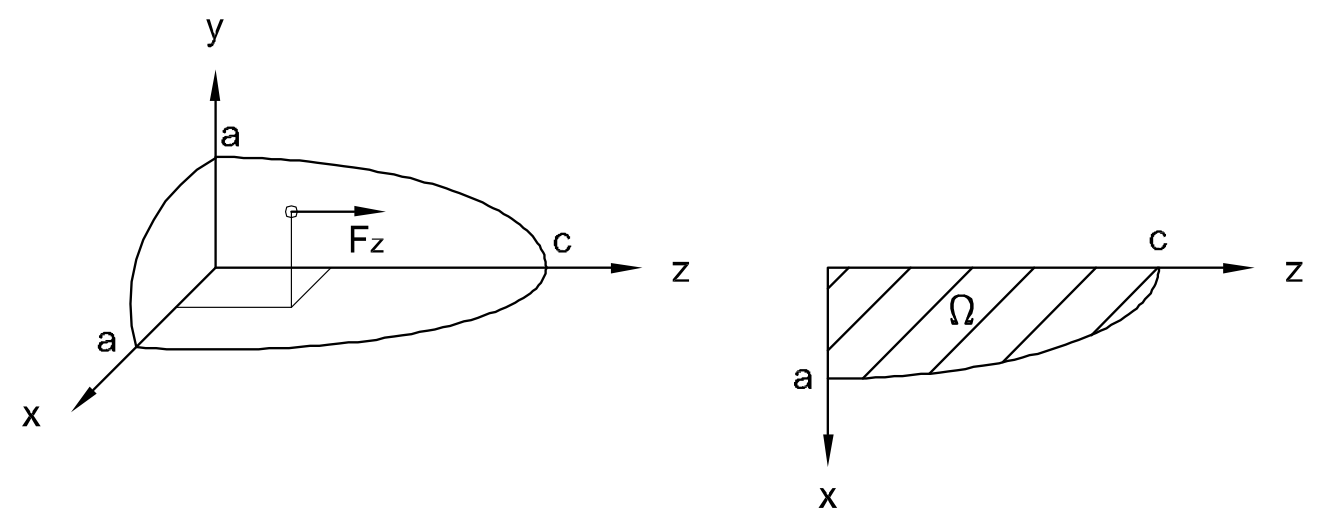

Figure C.1: Three-dimensional torque differential

As the surface traction $F_{z}$ is independent of $x, z$, it can be pulled out of the integral. The remaining integral multiplied by eight, is equivalent to the volume of the prolate spheroid.

$$
\text { Torque }=F_{z} 8 \iint_{\Omega} y(x, z) d x d z=F_{z} \text { Volume }
$$

Therefore, it can be said that the relation between the torque generated by the actuator and its volume is constant and equal to the value of the surface traction $F_{z}$,

$$
\frac{\text { Torque }}{\text { Volume }}=F_{z}
$$

\section{C.2 Two-dimensional case}

For the two-dimensional case, the geometry of the actuator is an elliptic cylinder. In this case, symmetry dictates that the calculation of the torque can be split in four parts.

$$
\text { Torque }=4 \int_{0}^{L} \int_{0}^{c} y(z) F_{z} d z d x
$$

where $L$ is the depth of the model.

Again, the surface traction $F_{z}$ is independent of $x, z$, it can be pulled out of the integral,

$$
\text { Torque }=F_{z} L 4 \int_{0}^{c} y(z) d z
$$

where $L 4 \int_{0}^{c} y(z) d z$ is the Volume of the actuator. Therefore, it is possible to express the relation between the torque generated by the elliptical cylinder shaped actuator and its volume as, 


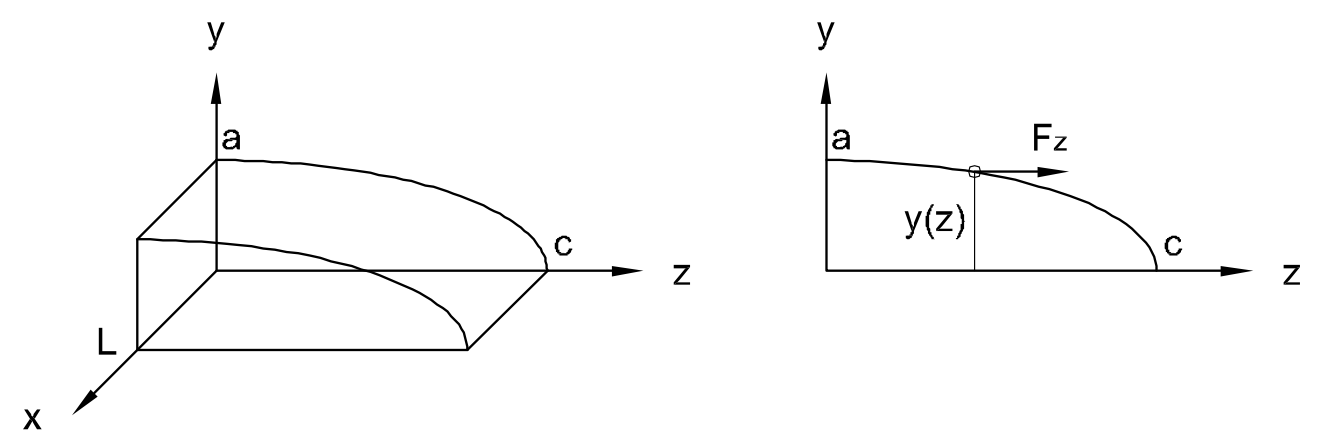

Figure C.2: Two-dimensional torque differential

$$
\frac{\text { Torque }}{\text { Volume }}=F_{z}
$$

From (C.5) and (C.8) it can be concluded that for a given surface traction $F_{z}$, the generated torque per unit volume of actuator is the same for the two- and three-dimensional cases. 


\section{Appendix D}

\section{Minimum RVE depth}

The process currently used to create the actuated polymer film, consists in the dispersion of liquid crystal droplets in a base film made of polyvinyl alcohol (PVA). After the PVA has dried, the film is stretched. In this stretched configuration, the film is exposed to ultra violet light to cure the liquid crystal actuators [42].

Assuming that the droplets were perfect spheres before the stretching and that when stretched and cured they become prolate spheroids, it is possible to calculate the minimum separation, in the depth direction, that two parallel actuators can have (See Figure D.1). An additional assumption must be made, that the volume of the liquid crystal does not changes when the film is stretched.

The volume of the initial spherical droplet is given by,

$$
\text { Volume }_{\text {sphere }}=\frac{4}{3} \pi r^{3}
$$

where $r$ is the radius of the sphere.

The volume of a prolate spheroid is given by,

$$
\text { Volume }_{\text {p.sph. }}=\frac{4}{3} \pi a^{2} c
$$

where $a, c$ are the minor and major radii, respectively.

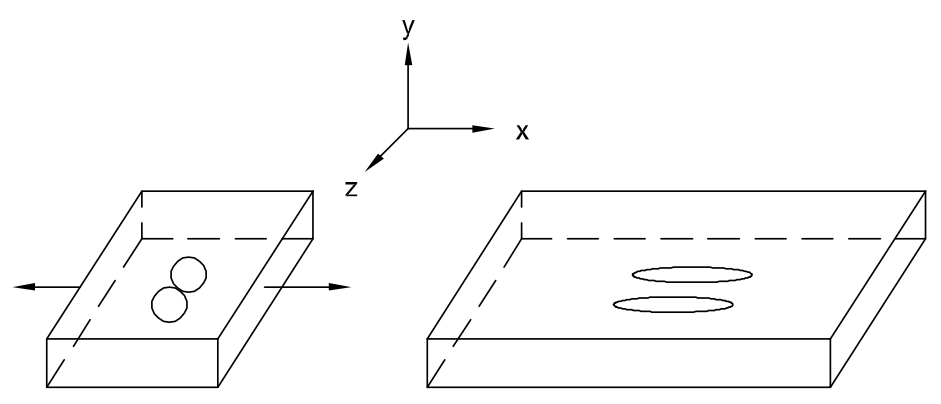

Figure D.1: Spherical droplets become prolate spheroids when stretched 


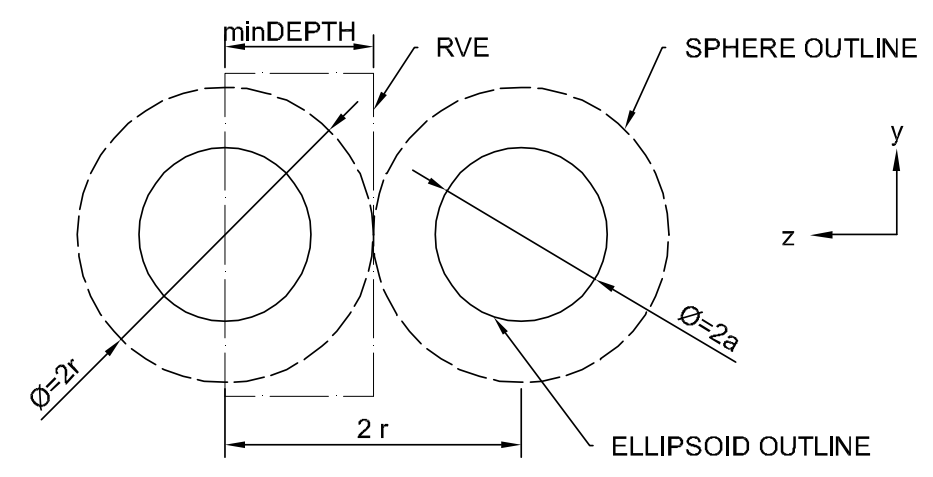

Figure D.2: Minimum theoretical depth of the RVE

If the volume of the liquid crystal droplet does not change during the stretching,

$$
\text { Volume }_{\text {p.sph. }}=\text { Volume }_{\text {sphere }}
$$

Therefore,

$$
r^{3}=a^{2} c
$$

Defining the aspect ratio AR of the prolate spheroid as,

$$
\mathrm{AR}=\frac{c}{a}
$$

and replacing this definition in (D.4), it is possible to find the radius of the original sphere as a function of the prolate spheroid minor radius $a$ and its aspect ratio AR,

$$
r=a \sqrt[3]{\mathrm{AR}}
$$

Under the aforementioned assumptions, in the limit case where two spherical droplets were cast in such way that they are tangential, the minimum depth for the RVE in the stretched configuration is given by (D.6), as it can be seen in Figure D.2. 


\section{Appendix E}

\section{ABAQUS Scripts}

\section{E.1 Contact Model - Partially Constrained Top Glass}

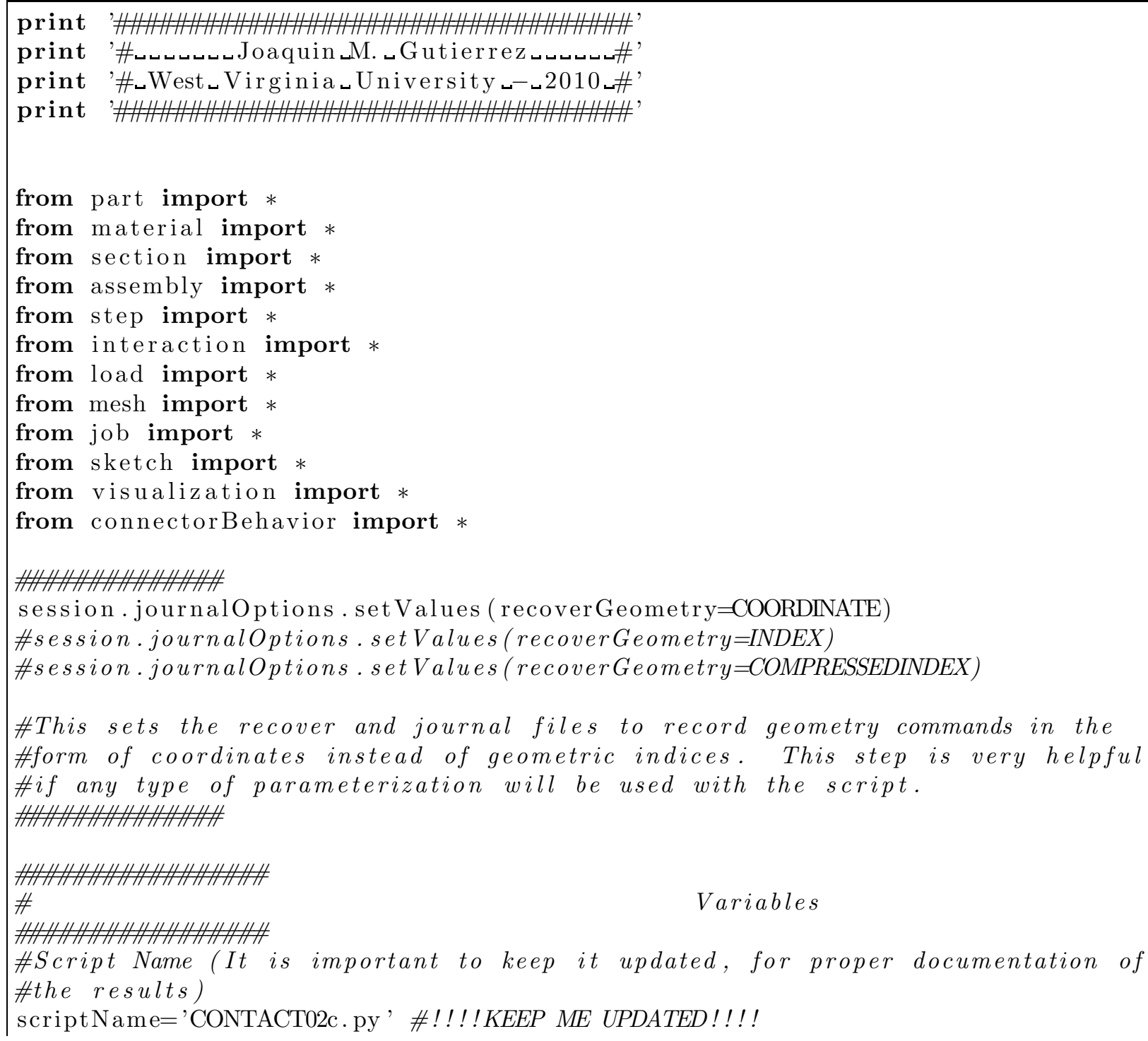




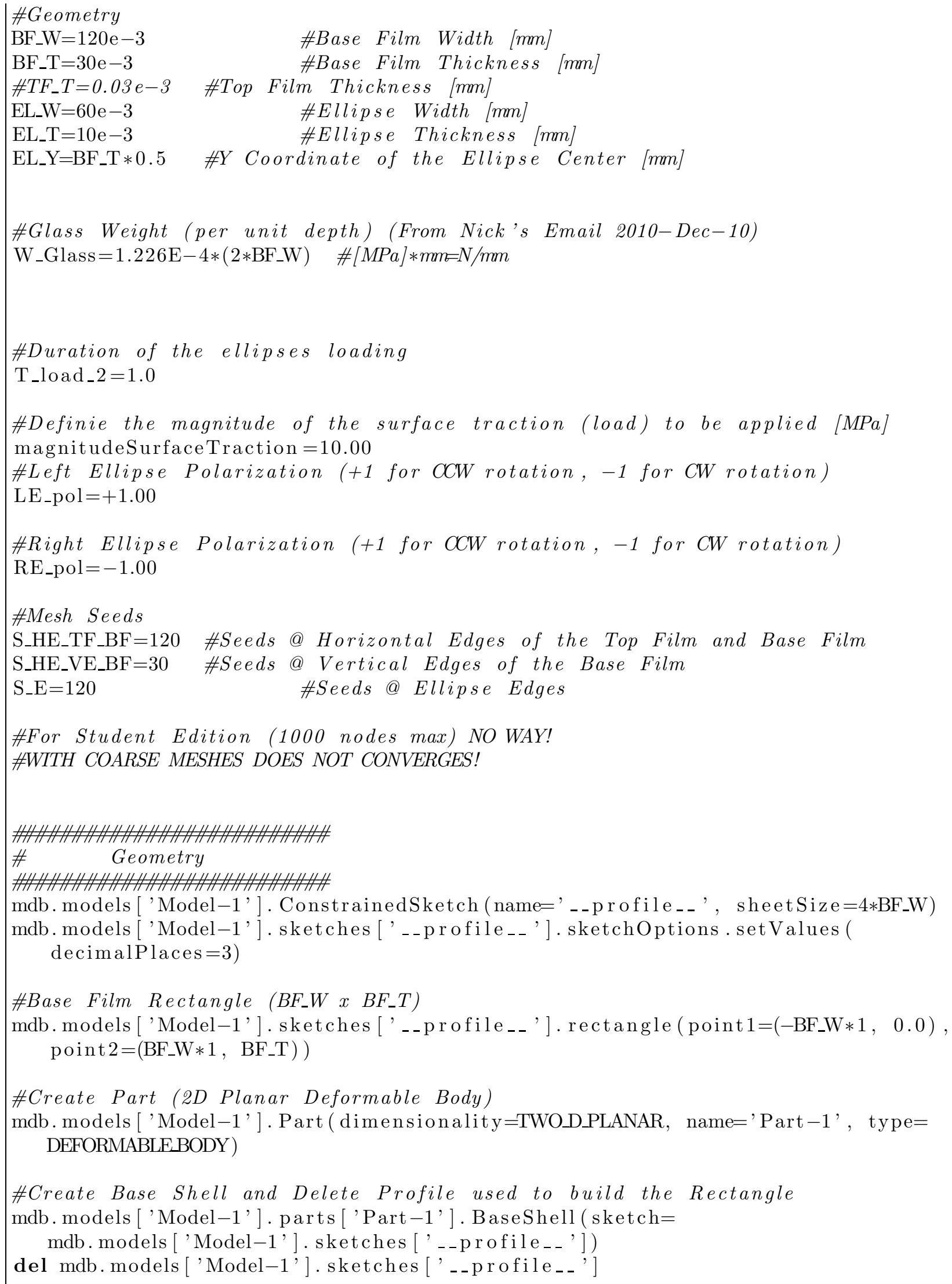


110

\#Partition of the LEFT and RIGHT Ellipses from the Rectangle Begins mdb. models ['Model-1'] . ConstrainedSketch (gridSpacing $=0.006$, name=' _-profile _-', sheetSize $=0.243$, transform $=$ mdb. models ['Model-1']. parts ['Part-1']. MakeSketchTransform( sketchPlane=mdb. models ['Model-1']. parts ['Part-1']. faces [0], sketchPlaneSide=SIDE1, sketchOrientation=RIGHT, origin $=(0.00,0.00,0.0)))$ mdb. models ['Model-1']. sketches [' _- profile__-']. sketchOptions . setValues ( decimalPlaces $=6$ )

mdb. models ['Model-1'] parts ['Part-1'] . projectReferences OntoSketch ( filter= COPLANAREDGES, sketch=mdb. models ['Model-1']. sketches [' _- profile _.']) \#Sketch Ellipse mdb. models ['Model-1'] . sketches [' - - profile_-_']. EllipseBy CenterPerimeter (

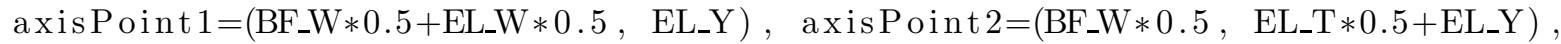
center $=\left(B_{-}-W * 0.5\right.$, EL_Y $\left.)\right)$

mdb. models ['Model-1'] . sketches [' --profile_--']. EllipseByCenterPerimeter( axisPoint $1=\left(-\mathrm{BF}_{-} \mathrm{W} * 0.5+\mathrm{EL} \_\mathrm{W} * 0.5\right.$, EL_Y $)$, axisPoint $2=\left(-\mathrm{BF} \_\mathrm{W} * 0.5, \quad\right.$ EL_T $* 0.5+$ EL_Y ) , center $\left.=\left(-\mathrm{BF}_{-} \mathrm{W} * 0.5, \mathrm{EL} \mathrm{L}_{-} \mathrm{Y}\right)\right)$

\#Do the actual Partition

mdb. models ['Model-1'] parts ['Part-1']. PartitionFaceBySketch (faces= mdb. models ['Model-1']. parts ['Part-1']. faces [0], sketch=mdb. models ['Model-1' ]. sketches [' - profile $_{-}$'] ])

del mdb. models ['Model-1']. sketches [' _- profile_.' ]

\#Partition of the LEFT and RIGHT Ellipses Ends

\#Partition of the LEFT Ellipse

mdb. models ['Model-1'] . parts ['Part-1']. PartitionEdgeByParam(edges= mdb. models ['Model-1'] . parts ['Part-1']. edges. find At $\left(\left(\left(-\mathrm{BF} \_\mathrm{W} * 0.5\right.\right.\right.$, EL_T $* 0.5+$ EL_Y, 0.0), ), ), parameter $=0.5$ )

\#Create of the LEFT Ellipse Top and Bottom Surfaces

mdb. models ['Model-1'] . parts ['Part -1 '] . Surface (name='Ellipse - Left - Top', side $2 \mathrm{Edges}=$ mdb. models ['Model -1 '] ]. parts ['Part -1 '] . edges . find At $(((-\mathrm{BF}-\mathrm{W} * 0.5$, EL_T $\left.\left.\left.* 0.5+E L \_Y, 0.0\right), \quad\right), \quad\right)$ )

mdb. models ['Model-1']. parts ['Part-1']. Surface (name='Ellipse-Left-Bottom ', side2Edges=mdb. models ['Model-1']. parts ['Part-1'] . edges . findAt $(((-B F+W * 0.5$, $\left.\left.\left.-\mathrm{EL} \_\mathrm{T} * 0.5+\mathrm{EL} \_\mathrm{Y}, 0.0\right), \quad\right), \quad\right)$ )

\#Partition of the RIGHT Ellipse

mdb. models ['Model-1'] . parts ['Part-1']. PartitionEdgeByParam(edges= mdb. models ['Model-1'] . parts ['Part-1'] . edges . find At ( ( $B$ B_W $* 0.5$, EL_T*0.5+ EL_Y, 0.0$),$ ), ) , parameter $=0.5)$

\#Create of the RIGHT Ellipse Top and Bottom Surfaces

mdb. models ['Model-1'] . parts ['Part-1'] . Surface (name='Ellipse-Right-Top', side 2 Edges $=$ mdb. models ['Model -1 '] . parts [ 'Part -1 ' $]$. edges . find At $(((\mathrm{BF}-\mathrm{W} * 0.5$, EL_T $\left.\left.\left.* 0.5+\mathrm{EL}_{-} \mathrm{Y}, 0.0\right), \quad\right), \quad\right)$ )

mdb. models ['Model-1']. parts ['Part-1']. Surface (name='Ellipse-Right-Bottom ', side 2 Edges $=$ mdb. models ['Model -1 ']. parts [ 'Part -1 '] . edges . find At ( ( BF_W*0.5, $\left.\left.\left.\left.-\mathrm{EL} \_\mathrm{T} * 0.5+\mathrm{EL} \_\mathrm{Y}, 0.0\right), \quad\right), \quad\right)\right)$

\#Instance Part-1-1 in the Assembly

mdb. models ['Model-1'] . root Assembly . DatumCsysByDefault (CARTESIAN)

mdb. models ['Model-1'] $\operatorname{root}$ Assembly. Instance (dependent=ON, name='Part-1-1', 
part $=$ mdb. models ['Model -1 '] . parts ['Part -1 '] )

mdb. models ['Model-1'] . rootAssembly.regenerate ()

\#Define Left and Right Surfaces

mdb. models ['Model-1']. parts ['Part-1']. Surface (name='LeftSurface', side1Edges= mdb. models ['Model-1'] . parts ['Part-1'] . edges. findAt $\left(\left(\left(-B F_{-} W\right.\right.\right.$, BF_T $\left.* 0.5,0.0\right)$ )$, \quad)$

mdb. models ['Model-1'] . parts ['Part-1']. Surface (name='RightSurface', side1Edges= mdb. models ['Model-1'] . parts ['Part-1'] . edges. find At ( ( BF_W, BF_T*0.5, 0.0), ))

\#Define TOP Surface

mdb. models ['Model-1']. parts ['Part-1']. Surface (name='Top', side1Edges= mdb. models ['Model-1'] . parts ['Part-1'] . edges . find At $\left(\left(\left(-B F_{-} W * 0.99\right.\right.\right.$, BF_T, $0.0)$, ), ))

\#Create Sets TOP, Bottom, Left, Right, PolymerMatrix

mdb. models ['Model-1'].parts ['Part -1 '] . Set ( faces= mdb. models ['Model-1'] . parts ['Part -1 '] . faces . findAt $\left(\left(\left(0, B F \_-T * 0.5\right.\right.\right.$, $0.0),(0.0,0.0,1.0)), \quad)$, name='PolymerMatrix')

mdb. models ['Model-1']. parts ['Part-1']. Set (edges= mdb. models ['Model-1'] . parts ['Part-1'] . edges. findAt $\left(\left(\left(-B F \_W, B F \_T * 0.5,0.0\right)\right.\right.$ ), ), name='Left' )

mdb. models ['Model-1'] parts ['Part-1']. Set (edges= mdb. models ['Model-1'] . parts ['Part-1'] . edges.find At ( ( BF_W, BF_T *0.5, 0.0), ), ), name='Right')

mdb. models ['Model-1'] . parts ['Part -1 '] . Set ( edges= mdb. models ['Model-1'] . parts ['Part-1'] . edges . findAt $(((0$, BF_T, 0.0), $)$, ), name='Top' )

mdb. models ['Model-1'] . parts ['Part-1']. Set (edges= mdb. models ['Model-1'] . parts ['Part-1'] . edges. findAt $(((0.0,0.0,0.0)), \quad$,$) ,$ name $=$ 'Bottom' )

mdb. models ['Model-1'] . rootAssembly . regenerate ()

\section{\#\#\#\#\#\#\#\#\#\#\#\#}

\#

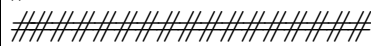

Displacement $B C^{\prime} s$

\#Displacement $B C$ 's

\#Bottom Edge $(U X=U Y=0)$

mdb. models ['Model-1']. DisplacementBC ( amplitude=UNSET, createStepName=' Initial' distributionType=UNIFORM, fieldName=', , localCsys=None, name='Bottom', region=Region ( edges $=$ mdb. models ['Model-1'] . rootAssembly . instances ['Part-1-1'] . edges . find At $(((0.0$, 
220

221

222

223

224

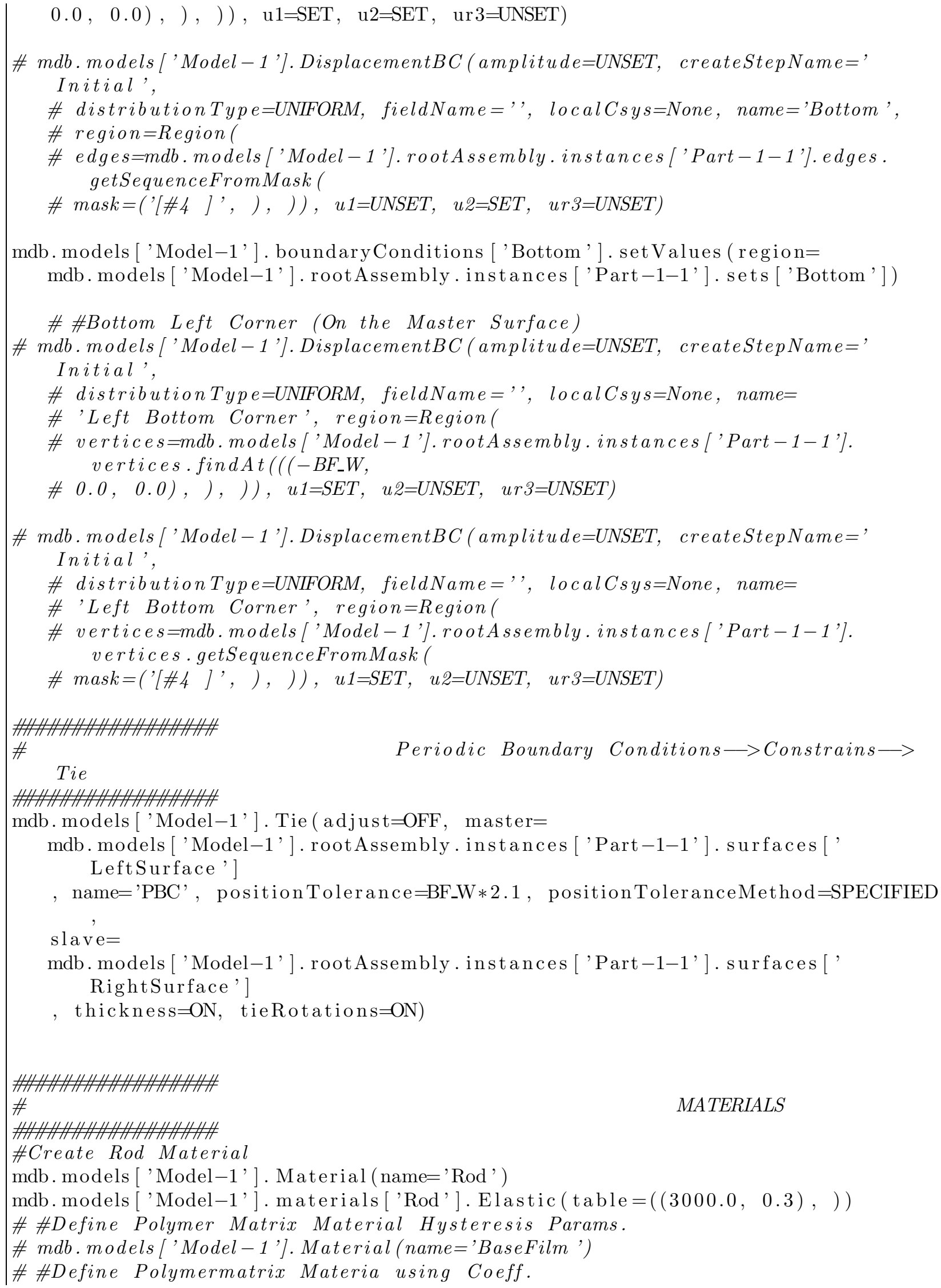




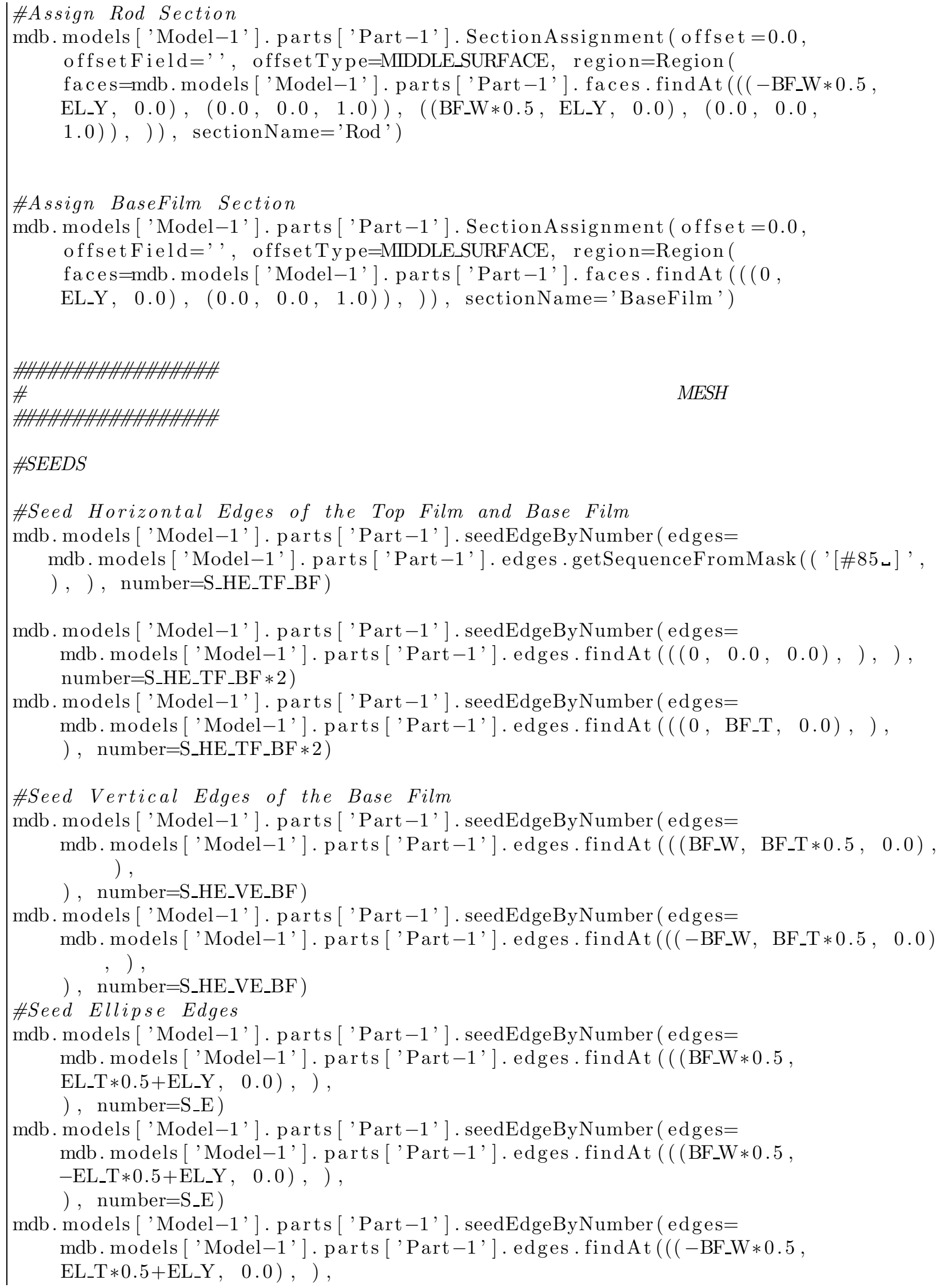


), number=S_E )

mdb. models ['Model-1'] . parts ['Part-1']. seedEdgeByNumber ( edges= mdb. models ['Model-1'] . parts ['Part-1'] . edges . find At $\left(\left(\left(-\mathrm{BF} \_\mathrm{W} * 0.5\right.\right.\right.$, $\left.\left.-\mathrm{EL} \_\mathrm{T} * 0.5+\mathrm{EL} \_\mathrm{Y}, 0.0\right), \quad\right)$,

), number=S_E )

\section{\#MESH CONTROL}

\#Mesh Controls on Base Film, Actuator)

mdb. models ['Model-1'] . parts ['Part-1']. setMeshControls (allowMapped=True, regions $=$ mdb. models ['Model -1 '] . parts ['Part -1 '] . faces . find At $(((-B F-W * 0.5$, EL_Y, 0.0$),(0.0,0.0,1.0)),\left(\left(\mathrm{BF}_{-} \mathrm{W} * 0.5\right.\right.$, EL_Y, 0.0$),(0.0,0.0$, $1.0)$ ), ))

mdb. models ['Model-1'] . parts ['Part-1'] . setMesh Controls (allowMapped=True, regions $=$ mdb. models ['Model -1 '] . parts ['Part -1 ' $]$. faces . find At $(((0$, EL_Y, 0.0$),(0.0,0.0,1.0)), \quad))$

\section{\#ELEMENT TYPES}

\#Element Type CPE4R,CPE3@Mask \#6: Faces 1,2 Top Film, Actuator \#Plane Strain Elements, Reduced Integration

mdb. models ['Model-1']. parts ['Part -1 '] . setElementType ( elem Types $=$ (ElemType ( elem Code $=$ CPEAR, elemLibrary $=$ STANDARD, secondOrderAccuracy $=\mathrm{OFF}$, hourglassControl=DEFAULT, distortionControl=DEFAULT), ElemType( elemCode=CPE3, elemLibrary $=$ STANDARD, distortionControl=DEFAULT $))$, regions $=($ mdb. models ['Model-1'] . parts ['Part -1 '] . faces . find At $(((-\mathrm{BF}-\mathrm{W} * 0.5$, EL_Y, 0.0$),(0.0,0.0,1.0)),\left(\left(\mathrm{BF}_{-} \mathrm{W} * 0.5\right.\right.$, EL_Y, 0.0$),(0.0,0.0$, $1.0)), \quad), \quad)$ )

\#Element Type CPE4RH, CPE3H @ Mask \#1: Face 0 (Base Film)

\#Hybrid, Plane Strain Elements, Reduced Integration \#NOTE: Hybrid elements are needed for INCOMPRESSIBLE material mdb. models ['Model-1'] . parts ['Part -1'] . setElementType ( elem Types $=($ ElemType ( elemCode=CPE4RH, elemLibrary=STANDARD, secondOrderAccuracy $=$ OFF, hourglassControl=DEFAULT), ElemType(elemCode=CPE3H, elemLibrary=STANDARD) ), regions $=($ mdb. models ['Model -1 '] . parts ['Part -1 '] . faces . find At $(((0$, EL_Y, 0.0$),(0.0,0.0,1.0)), \quad), \quad))$

\#CREATE DATUM PLANES FOR FURTHER PARTITIONS TO ALLOW STRUCTURED MESHING mdb. models ['Model-1'] . parts ['Part-1']. DatumPlaneByPrincipalPlane ( offset=BF_T $* 0.5$, principalPlan $=$ XZPLANE)

mdb. models ['Model-1'] . parts ['Part-1'] . DatumPlaneByPrincipalPlane( offset=BF_W $* 0.5$, principalPlan $\mathrm{e}=$ YZPLANE) 
mdb. models ['Model-1'] . parts ['Part-1'] . DatumPlaneByPrincipalPlane( offset=-BF_W $* 0.5$, principalPlan $=$ YZPLANE)

mdb. models ['Model-1'] . parts ['Part-1']. DatumPlaneByPrincipalPlane ( offset $=0.0$, principalPlan $=$ YZPLANE)

\#Switch to COMPRESSED INDEX to do the partitions...these could be defined using "findat" if needed

session . journalOptions. setValues (recoverGeometry=COMPRESSEDINDEX)

\#CREATE FURTHER PARTITIONS TO ALLOW STRUCTURED MESHING

mdb. models ['Model-1'] . parts ['Part -1 '] . PartitionFaceByDatumPlane (datumPlane= mdb. models ['Model-1']. parts ['Part-1']. datums [32], faces= mdb. models ['Model-1'] . parts ['Part-1']. faces . getSequenceFromMask(('[\#7_] ', )$, \quad)$ )

mdb. models ['Model-1'] . parts ['Part-1']. PartitionFaceByDatumPlane (datumPlane= mdb. models ['Model-1'] . parts ['Part -1 '] . datums [34], faces= mdb. models ['Model-1'] . parts ['Part-1']. faces . getSequenceFromMask(('[\#3f $\left.\mathrm{f}_{\mathbf{L}}\right]$ ', )$, \quad)$

mdb. models ['Model-1'] . parts ['Part -1 '] . PartitionFaceByDatumPlane (datumPlane $=$ mdb. models ['Model-1']. parts ['Part-1']. datums [35], faces= mdb. models ['Model-1'] parts ['Part-1']. faces . getSequenceFromMask (('[\#3ff _ ]' ), )

mdb. models ['Model-1'] . parts ['Part-1'] . PartitionFaceByDatumPlane (datumPlane= mdb. models ['Model-1']. parts ['Part -1 ']. datums [33], faces= mdb. models ['Model-1']. parts ['Part-1']. faces .getSequenceFromMask(('[\#fff _ ]' )$, \quad)$

\section{\#SET STRUCTURED MESHING}

mdb. models ['Model-1'] . parts ['Part-1'] . setMeshControls (regions= mdb. models ['Model-1'] . parts ['Part-1'] f faces . getSequenceFromMask (( '[\#ffff $]$ ' ', ), ), technique=STRUCTURED)

\section{\#GENERATE MESH}

mdb. models ['Model-1'] . parts ['Part-1']. generateMesh ()

mdb. models ['Model-1']. rootAssembly.regenerate ()

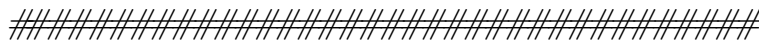

\# Create Analytical Surface (TOP GLASS)

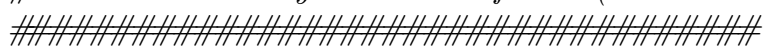

mdb. models ['Model-1'] . ConstrainedSketch (name=' _- profile _-', sheetSize $=0.5)$

mdb. models ['Model-1'] . sketches [' _- profile_-'] . Line ( point1= $\left(-\mathrm{BF}_{-} \mathrm{W} * 1.1,0.0\right)$, point $2=$

$\left.\left(\mathrm{BF} \_\mathrm{W} * 1.1,0.0\right)\right)$

mdb. models ['Model-1']. sketches [' --profile_-']. HorizontalConstraint (entity= mdb. models ['Model-1']. sketches [' _- profile__'] . geometry. findAt $\left(\left(\begin{array}{lll}0.0 & 0.0\end{array}\right)\right.$, ))

mdb. models ['Model-1'] . Part (dimensionality=TWO_D_PLANAR, name='Part-2', type= ANALYTIC_RIGID_SURFACE) 
mdb. models ['Model-1'] . parts ['Part-2']. AnalyticRigidSurf2DPlanar (sketch= mdb. models ['Model-1']. sketches [' - - profile - $_{-}$'])

del mdb. models ['Model-1']. sketches [' _- profile_.'']

\#Define the Reference Point

mdb. models ['Model-1'] . parts ['Part-2']. ReferencePoint (point $=\left(\begin{array}{llll}0.0,0.0,0.0\end{array}\right)$

\#Define Surface

mdb. models ['Model-1'] . parts ['Part-2'] . Surface (name='Glass-Surface', side2Edges $=$ mdb. models ['Model-1'] . parts ['Part-2'] . edges . find At $(((-0.5 *$ BF_W, $0.0,0.0)$, ),

))

mdb. models ['Model-1']. rootAssembly.regenerate ()

\#Move Instance to the top of the film

mdb. models ['Model-1'] . rootAssembly. Instance (dependent $=0 N$, name='Part $-2-1$ ', part $=$ mdb. models ['Model -1 '] . parts ['Part -2 '] )

mdb. models ['Model -1 '] . rootAssembly . translate (instanceList $=($ 'Part $-2-1$ ', $)$, vector $\left.=\left(0.0, \mathrm{BF}_{-} \mathrm{T} * 1.20,0.0\right)\right)$

\#Constrain the glass to be in contact with the film

mdb. models ['Model-1'] $\operatorname{root}$ Assembly.EdgeToEdge (clearance $=0.0$, fixed Axis $=$ mdb. models ['Model-1'] . rootAssembly. instances ['Part-1-1'] . edges . find At ( ( $-0.9 *$ BF_W, BF_T, 0.0$),$ ), flip=OFF, movableAxis $=$ mdb. models ['Model-1'] . rootAssembly. instances ['Part-2-1'] . edges . find At ( ( $\left.\left.\left.-0.9 * \mathrm{BF}_{-} \mathrm{W}, \mathrm{BF} \_\mathrm{T} * 1.20,0.0\right), \quad\right)\right)$

\#Apply $B C$ to the Top Glass (UX=ROTZ=0)

mdb. models ['Model-1']. DisplacementBC (amplitude=UNSET, createStepName=' Initial' distributionType=UNIFORM, fieldName=', , localCsys=None, name='BC-3', region=Region ( referencePoints $=($

mdb. models ['Model-1'] rootAssembly.instances ['Part-2-1'] r referencePoints $[2]$,

)) , u1=SET, u2=UNSET, ur $3=$ SET)

\#Create Step-1: Where the glass weight will be applied

mdb. models ['Model-1']. StaticStep (description='Apply॰Glass_Weight', name=

'Step-1', nlgeom=ON, previous='Initial')

\#Apply Glass Weight Load

mdb. models ['Model-1'] . ConcentratedForce ( cf2 $=-1.0 *$ W_Glass, createStepName $=$

'Step-1', distributionType=UNIFORM, field=', , localCsys=None, name='Load-1

, region=Region $($ referencePoints $=($

mdb. models ['Model-1'] rootAssembly. instances ['Part-2-1']. referencePoints

)) )

$[2]$

\#Create Step-2: Where the ellipses are loaded (constant load, DC voltage)

mdb. models ['Model-1']. StaticStep (description=

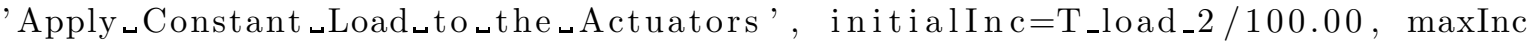
$=\mathrm{T}_{-} \mathrm{load}_{-} 2$,

minInc $=1 / 1000000.00 * T_{\_}$load_2, name='Step -2 ', previous='Step -1 ', timePeriod $=\mathrm{T}_{-} \mathrm{load}_{-} 2$ ) 
472

473

\#Apply Load on Ellipse-Left-Top

mdb. models ['Model-1']. SurfaceTraction (createStepName='Step-2', directionVector

$((0.0,0.0,0.0),(1.0,0.0,0.0))$, distributionType=UNIFORM, field=',

follower $=$ OFF, localCsys=None, magnitude $=-1.00 *$ LE_pol $*$ magnitudeSurfaceTraction, name=

'Load-Ellipse-Left-Top', region=

mdb. models ['Model-1']. rootAssembly. instances ['Part-1-1']. surfaces ['Ellipse - Left-Top']

, traction=GENERAL)

\#Apply Load on Ellipse-Left-Bottom

mdb. models ['Model-1'] . SurfaceTraction (createStepName='Step-2', directionVector $=$

$((0.0,0.0,0.0),(1.0,0.0,0.0))$, distributionType=UNIFORM, field=', ,

follower=OFF, localCsys=None, magnitude=1.00*LE_pol* magnitudeSurfaceTraction, name=

'Load-Ellipse-Left-Bottom', region=

mdb. models ['Model-1'] . rootAssembly. instances ['Part-1-1']. surfaces ['Ellipse -Left-Bottom']

, traction=GENERAL)

\#Apply Load on Ellipse-Right-Top

mdb. models ['Model-1']. SurfaceTraction (createStepName='Step-2', directionVector $=$

$((0.0,0.0,0.0),(1.0,0.0,0.0))$, distributionType=UNIFORM, field=',

follower $=\mathrm{OFF}$, localCsys=None, magnitude $=-1.00 * \mathrm{RE} \_$pol $*$ magnitudeSurfaceTraction, name=

'Load-Ellipse-Right-Top', region=

mdb. models ['Model-1'] . rootAssembly. instances ['Part-1-1']. surfaces ['Ellipse -Right-Top']

, traction=GENERAL)

\#Apply Load on Ellipse-Right-Bottom

mdb. models ['Model-1']. SurfaceTraction (createStepName='Step-2', directionVector $=$

$((0.0,0.0,0.0),(1.0,0.0,0.0))$, distributionType=UNIFORM, field=',

follower=OFF, localCsys=None, magnitude=1.00*RE_pol $*$ magnitudeSurfaceTraction, name=

'Load-Ellipse-Right-Bottom', region= mdb. models ['Model-1'] . rootAssembly. instances ['Part-1-1']. surfaces ['Ellipse -Right-Bottom']

, traction=GENERAL)

\#Create Interaction Property for the Contact betwen Glass and Film ( Frictionless)

mdb. models ['Model-1'] . ContactProperty ('NOFric')

mdb. models ['Model-1']. interactionProperties ['NOFric']. TangentialBehavior ( formulation=FRICTIONLESS )

\#Define Contact Properties

\#WARNING: There are many parameters to play with here!!!

\# mdb. models ['Model-1']. SurfaceToSurfaceContactStd (adjustMethod=NONE,

\# clearanceRegion=None, createStepName='Initial', datumAxis=None, enforcement= 


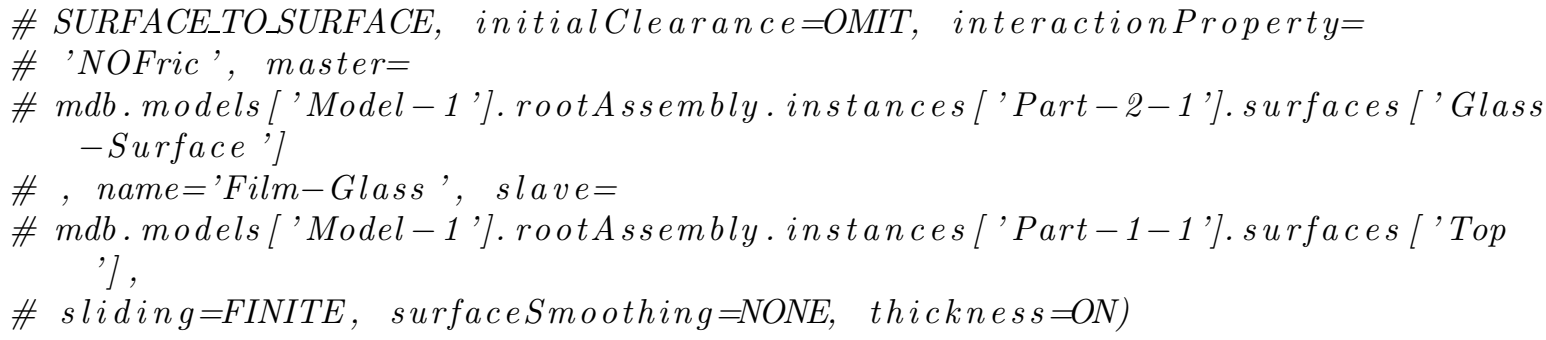




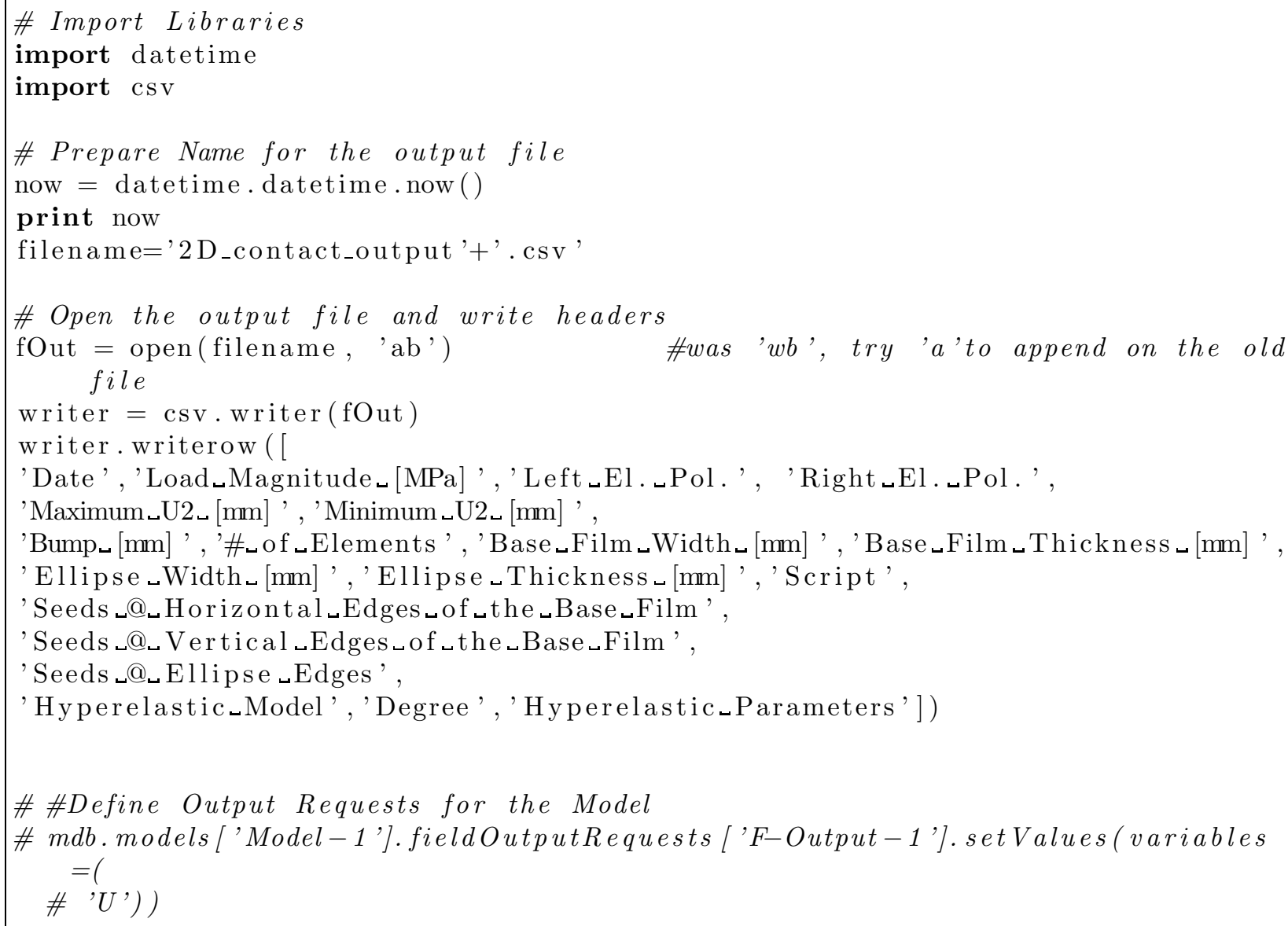


635

minimo $=0.0$

for JJ in range (0,len(frameU.values)): \#Loop over nodes if frameU.values [JJ].data[1]>maximo: maximo=frameU . values $[\mathrm{JJ}]$. data $[1]$

for JJ in range( 0 , len (frameU.values)): \#Loop over nodes if frameU.values [JJ].data $[1]<$ minimo: minimo=frameU. values $[\mathrm{JJ}]$. data $[1]$

print maximo

\# print minimo

now $=$ datetime.datetime.now ()

print now

\# Get Number of elements in the model

numberElements=mdb . models [ 'Model-1'] . rootAssembly . verify MeshQuality ( ANALYSIS_CHECKS) [ 'numElements']

\# Write the results for the current frequency to the output file writer.writerow ([

now.strftime ("\%Y-\%m-\%d_\%H:\%M:\%S"), str ( magnitudeSurfaceTraction ), str (LE_pol), str (RE_pol), str (maximo), str (minimo), str (maximo-minimo),

numberElements ,BF_W, BF_T,EL_W, EL_T, scriptName ,

S_HE_TF_BF , S_HE_VE_BF, S_E ,

odb. materials [ 'BASEFLL'] . hyperelastic.type, odb. materials [ 'BASEFILM'] . hyperelastic.n,

odb. materials ['BASEFILM']. hyperelastic.table])

\# Close the $O D B$ for the current frequency

odb.close ()

\# Close The output file

fOut. close ()

print "\#\#\#\#\#\#\#\#\#\#"

print "\#_Analysis _Done_\#"

print "\#\#\#\#\#\#\#\#\#\#\#"

\section{E.2 Two-Dimensional Model}

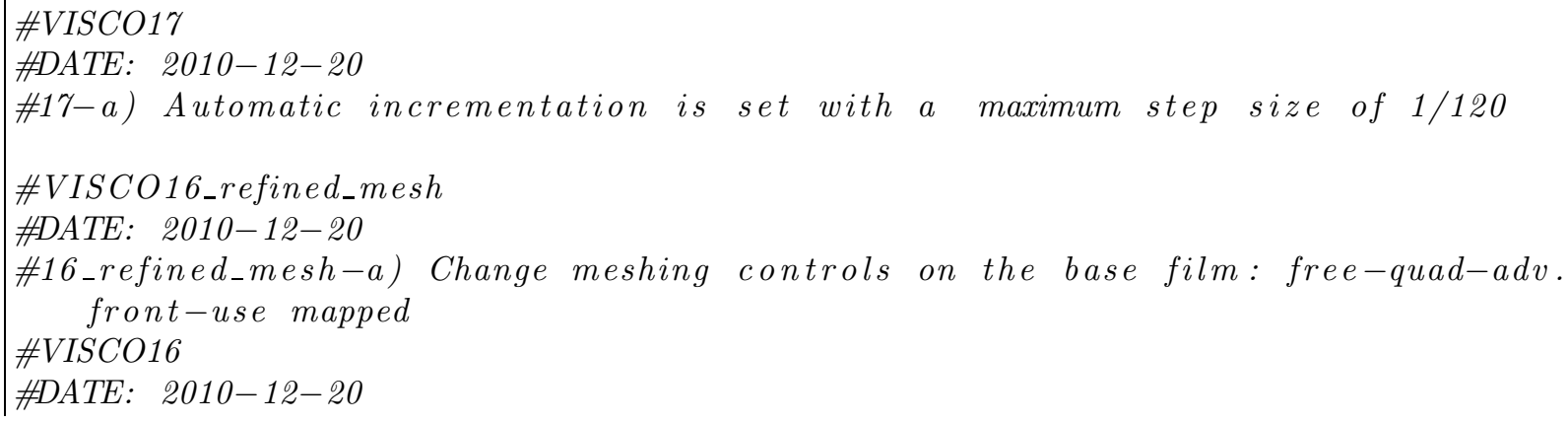




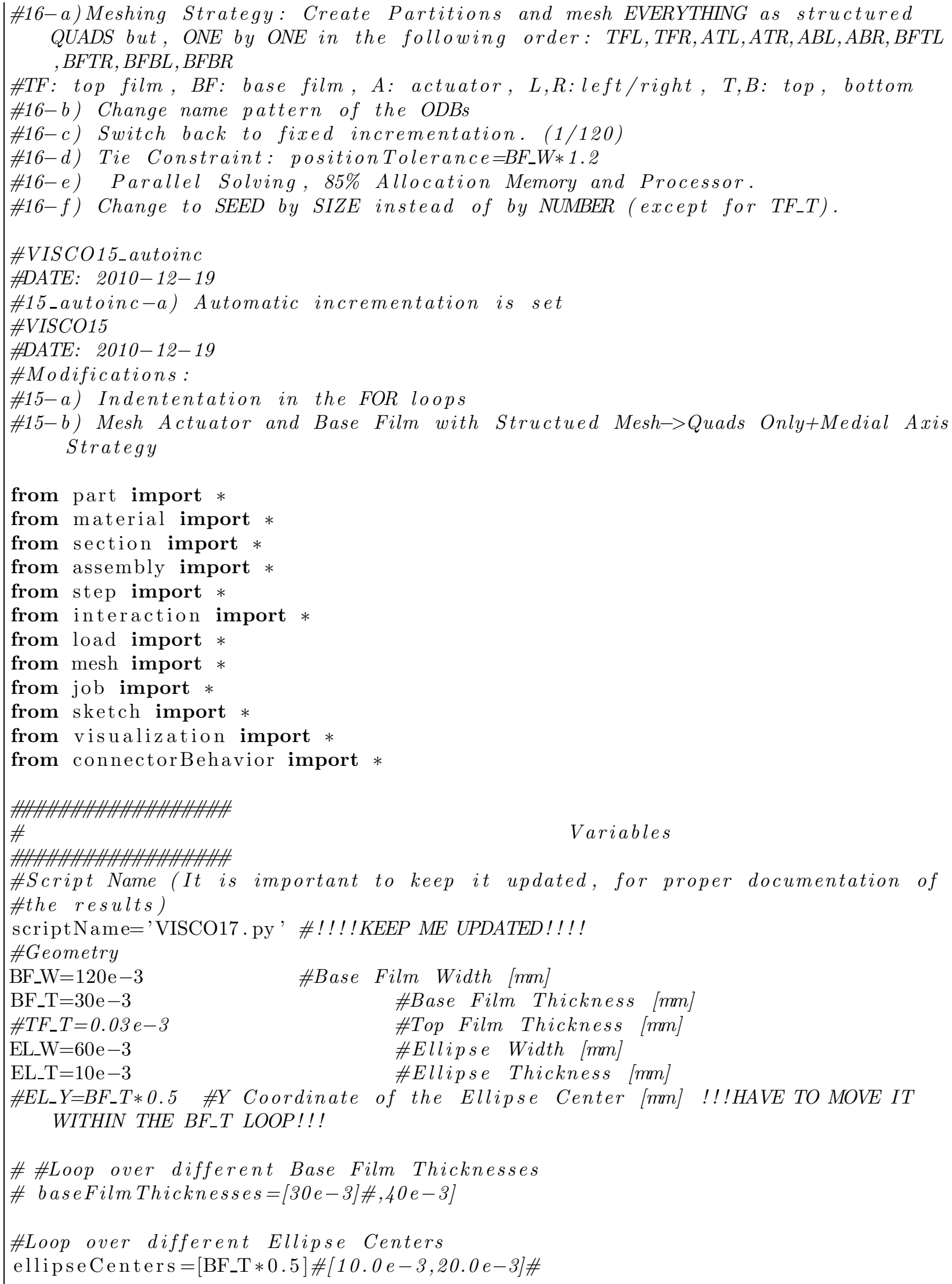




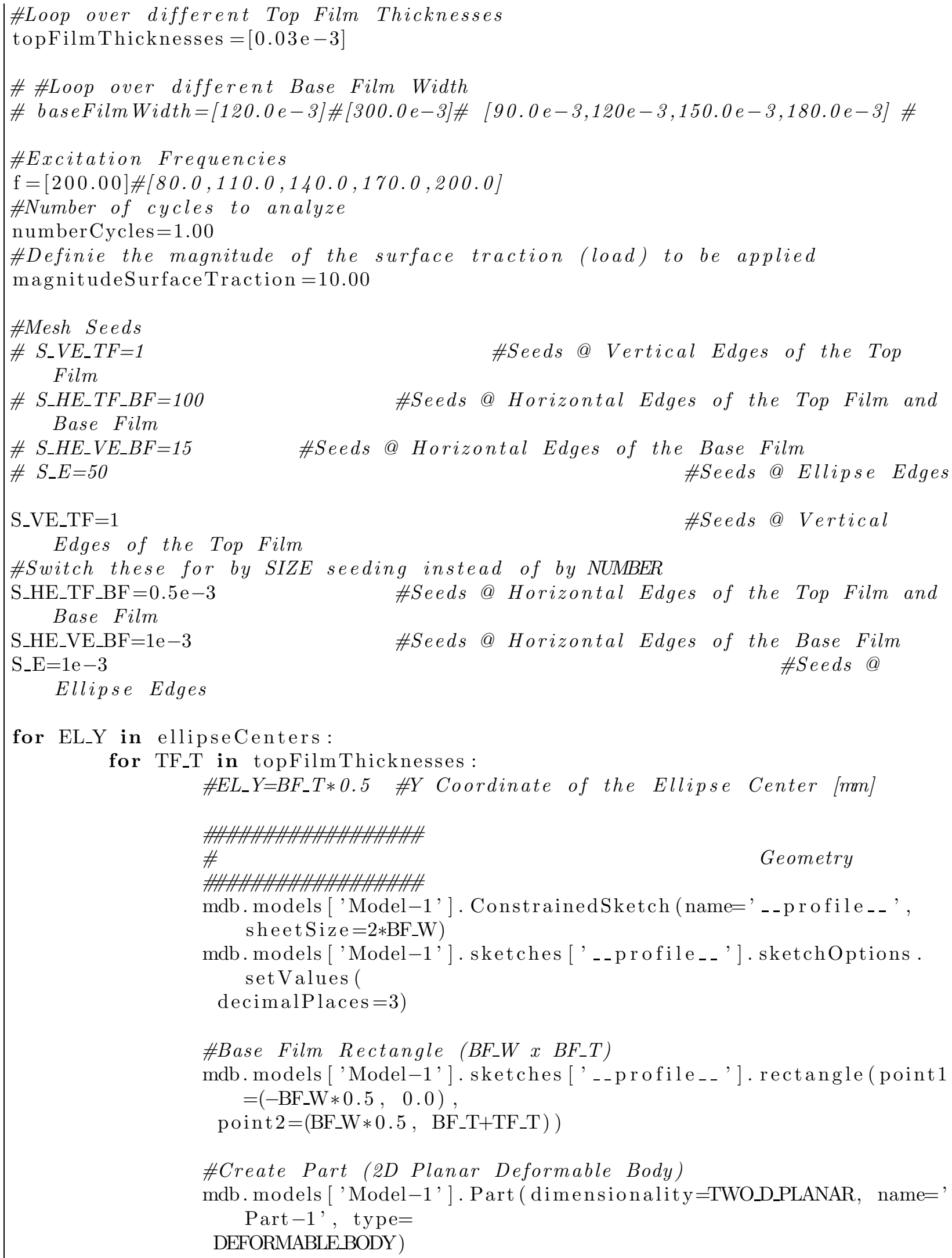


\#Create Base Shell and Delete Profile used to build the Rectangle

mdb. models ['Model-1']. parts ['Part -1 '] . BaseShell ( sketch= mdb. models ['Model-1']. sketches [' _- profile_--'])

del mdb. models['Model-1']. sketches [' -_profile_-']

\#Partition of the Top Film Begins

\#Create Sketch on the rectangle created before (Should be Face "0")

mdb. models ['Model-1'] . ConstrainedSketch ( gridSpacing $=0.006$, name $=$ ' - - profile $\ldots$ ',

sheetSize $=2 *$ BF_W, transform $=$

mdb. models ['Model-1'] . parts ['Part-1']. MakeSketchTransform( sketchPlane $=$ mdb. models ['Model-1']. parts ['Part -1 '] . faces [0], sketchPlaneSide=SIDE1, sketchOrientation $=$ RIGHT, origin $=(0.00$, 0.00

$0.0))$ )

mdb. models ['Model-1']. sketches [' _- profile__-']. sketchOptions. setValues (

decimalPlaces $=6$ )

mdb. models ['Model-1'] . parts ['Part -1 '] . projectReferencesOntoSketch (filter $=$

COPLANAREDGES, sketch=mdb. models ['Model-1']. sketches [' -_profile__' ])

\#Sketch Line

mdb. models ['Model-1'] . sketches [' -_profile__-']. Line ( point $1=(-$ BF_W $* 0.5$, BF_T $)$,

point $2=\left(\mathrm{BF} \_\mathrm{W} * 0.5\right.$, BF_T $\left.)\right)$

\#Do the Actual Partition

mdb. models ['Model-1'] . parts ['Part-1'] . PartitionFaceBySketch ( faces $=$

mdb. models ['Model-1'] . parts ['Part-1'] . faces [0], sketch=mdb. models ['Model-1']. sketches [' _- profile _.'])

del mdb. models ['Model-1']. sketches [' _- profile_.' ']

\#Partition of the Top Film Ends

\#Partition of the Ellipse from the Rectangle Begins

mdb. models ['Model-1'] . ConstrainedSketch ( gridSpacing $=0.006$, name $=$ ' - profile pro $_{-}$,

sheetSize $=0.243$, transform $=$

mdb. models ['Model-1'] . parts ['Part-1'] . MakeSketchTransform ( sketchPlane $=$ mdb. models ['Model-1'] . parts ['Part-1']. faces [0], sketchPlaneSide=SIDE1, sketchOrientation $=$ RIGHT, origin $=(0.00$, $0.00,0.0))$ )

mdb. models ['Model-1']. sketches [' _- profile__-']. sketchOptions. setValues (

decimalPlaces $=6)$

mdb. models ['Model-1']. parts ['Part-1'] . projectReferencesOntoSketch (filter=

COPLANAREDGES, sketch=mdb. models ['Model-1'] . sketches [' -_profile__' ])

\#Sketch Ellipse

mdb. models ['Model-1'] . sketches [' -_profile_-'] .

EllipseBy CenterPerimeter( 
axisPoint $1=\left(\mathrm{EL}_{-} \mathrm{W} * 0.5, \mathrm{EL} \_\mathrm{Y}\right)$, axisPoint $2=\left(0.0, \mathrm{EL} \_\mathrm{T} * 0.5+\mathrm{EL}_{-} \mathrm{Y}\right)$, center $=(0.0$, EL_Y $))$

\#Do the actual Partition

mdb. models ['Model-1']. parts ['Part-1']. PartitionFaceBySketch ( faces $=$

mdb. models ['Model-1']. parts ['Part -1 '] . faces [0], sketch=mdb. models ['Model-1']. sketches [' -_profile__' ])

del mdb. models ['Model-1']. sketches ['_-_profile_-'] \#Partition of the Ellipse Ends

\#Instance Part-1-1 in the Assembly

mdb. models ['Model-1'] . rootAssembly . DatumCsysByDefault ( CARTESIAN)

mdb. models ['Model-1'] . rootAssembly. Instance (dependent $=\mathrm{ON}$, name $={ }^{\prime}$ Part $-1-1$ ',

part $=$ mdb. models [ 'Model -1 '] $\cdot$ parts [ 'Part -1 '] $)$

mdb. models ['Model-1'] . rootAssembly. regenerate ()

\#Partition the Ellipse Edge in halves

mdb. models ['Model-1'] . parts ['Part-1'] . PartitionEdgeByParam( edges $=$

mdb. models ['Model-1'] . parts ['Part-1'] . edges . getSequenceFromMask(('[\#10_] ',

), ), parameter $=0.5)$

mdb. models ['Model-1']. parts ['Part-1']. regenerate ()

\#Define Ellipse-BottomSurface and TopSurface

mdb. models ['Model-1'] . parts ['Part-1']. Surface (name=' Ellipse-

BottomSurface', side2Edges=

mdb. models ['Model-1'] . parts ['Part-1'] . edges . getSequenceFromMask(('[\#10_ _ , ,

)$, \quad)$

mdb. models ['Model-1'] . parts ['Part-1'] . Surface (name=' EllipseTopSurface', side2Edges=mdb. models ['Model-1'] . parts ['Part-1']. edges . getSequenceFromMask (

$\left.\left.\left({ }^{\prime}\left[\# 20_{\bullet}\right] ', \quad\right), \quad\right)\right)$

\#Define Left and Right Surfaces

mdb. models ['Model-1'] . parts ['Part-1']. Surface (name='

LeftSurface', side1Edges=

mdb. models ['Model-1'] . parts ['Part-1']. edges . getSequenceFromMask( ('[\#102 ـ ]',

)$, \quad)$ )

mdb. models ['Model-1']. parts ['Part-1']. Surface (name='

RightSurface', side1Edges= mdb. models ['Model-1'] . parts ['Part-1']. edges . getSequenceFromMask ( ('[\#48 $]$ ', )$, \quad)$ )

\#Create Sets TOP, Bottom, Left, Right, PolymerMatrix mdb. models ['Model-1'] parts ['Part-1']. Set (edges= 
mdb. models ['Model-1']. parts ['Part-1']. edges . getSequenceFromMask ( ('[\#4_ $]$ ',

), ), name='Bottom' )

mdb. models ['Model-1'] . parts ['Part-1']. Set (edges= mdb. models ['Model-1']. parts ['Part-1']. edges . getSequenceFromMask (('[\#80_ ] ',

), ), name='Top')

mdb. models ['Model-1'] parts ['Part -1 '] . Set (edges= mdb. models ['Model-1']. parts ['Part-1']. edges . getSequenceFromMask ( ('[\#102 ـ ] ,

), ) , name='Left')

mdb. models ['Model-1'] parts ['Part-1']. Set (edges= mdb. models ['Model-1'] . parts ['Part-1']. edges . getSequenceFromMask ( ('[\#48 $]_{\text {ـ }}$ ',

), ), name='Right')

mdb. models ['Model-1'] . parts ['Part -1 '] . Set ( faces= mdb. models ['Model-1']. parts ['Part-1']. faces . getSequenceFromMask(('[\#1_u]',

), ), name='PolymerMatrix')

mdb. models ['Model-1']. rootAssembly.regenerate ()

\#Redefine Surfaces, Sets Using Geometry Variables and findAt \#L eft $S$ et

mdb. models ['Model-1'] parts ['Part -1 '] . Set (edges= mdb. models ['Model-1'] . parts ['Part-1'] . edges . find At $\left(\left(\left(-B_{-} W / 2\right.\right.\right.$, BF_T $/ 2,0.0)$

), ), name='Left')

\#Right Set

mdb. models ['Model-1'] . parts ['Part-1']. Set (edges=

mdb. models ['Model-1'] . parts ['Part-1'] . edges . find At ( ( BF_W/2, BF_T $/ 2,0.0), \quad)$,

), name='Right' )

\#Top Set

mdb. models ['Model-1'] . parts ['Part -1 '] . Set (edges= mdb. models ['Model-1'] . parts ['Part-1'] . edges . find At ( ( 0.0 , BF_T+TF_T, 0.0),

), ), name='Top')

\#Ellipse Bottom Surface

mdb. models ['Model-1'] . parts ['Part-1'] . Surface (name=' Ellipse -

BottomSurface',

side2Edges=mdb. models ['Model-1']. parts [ 'Part -1 '] . edges . find At ( ( $(0.0$

EL_Y-EL_T $/ 2,0.0), \quad), \quad))$

\#Ellipse Top Surface

mdb. models ['Model-1'].parts ['Part-1']. Surface (name='Ellipse-

TopSurface',

side2Edges=mdb. models ['Model -1 '] . parts ['Part -1 '] . edges . find At $(((0.0)$

EL_Y+EL_T $/ 2,0.0), \quad), \quad))$

\#Left Surface

mdb. models ['Model-1']. parts ['Part-1']. Surface (name='

LeftSurface', side1Edges=

mdb. models ['Model-1'] . parts ['Part-1'] . edges . find At $(((-B F-W / 2$, BF_T $/ 2,0.0)$, 
\#Create Top Film Material

mdb. models ['Model-1']. Material (name='TopFilm')

mdb. models ['Model-1']. materials ['TopFilm']. Elastic (table $=((70000.0,0.3), \quad))$

\#Create Rod Material

mdb. models ['Model-1'] . Material (name='Rod')

mdb. models ['Model-1'] . materials ['Rod'] . Elastic (table $=((3000.0$, $0.3), \quad)$ )

\#Define Polymer Matrix Material Hysteresis Params.

mdb. models ['Model-1']. Material (name='BaseFilm')

\#Define Polymermatrix Materia using Coeff.

\# mdb. models ['Model-1']. materials ['BaseFilm']. Hyperelastic ( $n$ $=2$, table $=(($

\#-30.8732, 41.5025, 10.3921, - 32.8395, 36.4617, 0.0, 0.0), ) , testData=OFF,

\# type=POLYNOMIAL, volumetricResponse=VOLUMETRIC_DATA)

\# mdb. models ['Model-1']. materials ['BaseFilm']. hyperelastic. Hysteresis (

\# table $=((2.0,0.5,4.5,-1.0), \quad))$

\#Define Base Film Material using Coefficients for ARRUDA-BOYCE MODEL (8-Chain)

\#Hytrel 4056 (TPE)

mdb. models ['Model-1']. Material (name='BaseFilm')

\#Units in $N, m m$

mdb. models ['Model-1']. materials ['BaseFilm']. Hyperelastic(table $=(($

$4.68032146,5311.53,0.0)$, ), testData=OFF, type $=$ ARRUDA_BOYCE,

volumetricResponse $=$ VOLUMETRICDATA)

\# mdb. models ['Model-1']. materials ['BaseFilm']. hyperelastic. UniaxialTestData(

\# table $=((2.83,0.0471),(4.29,0.0833),(5.5,0.127)$, (6.2, $0.163),(6.83$,

\# 0.207), (7.21, 0.243), (7.55, 0.286), (7.74, 0.323), $(7.92,0.366),(8.02$,

\# 0.402), (8.09, 0.446), (8.15, 0.482), (8.18, 0.525), (8.2, $0.562),(8.23$,

$\# 0.605),(8.25,0.641),(8.29,0.685),(8.32,0.721)$, $(8.36,0.765),(8.39$

$\# 0.801),(8.44,0.844),(8.48,0.881),(8.53,0.924)$, $(8.57,0.96),(8.62$

$\# 1.0),(8.69,1.05),(8.73,1.08),(8.79,1.13),(8.84$, $1.16),(8.91,1.21)$,

$\#(8.96,1.24),(9.03,1.29),(9.09,1.32),(9.17,1.37)$, $(9.22,1.4),(9.3$,

$\# 1.45),(9.37,1.48),(9.45,1.53),(9.52,1.56),(9.6$, $1.61),(9.68,1.64)$,

$\#(9.78,1.68),(9.86,1.72),(10.0,1.76),(10.1,1.8)$, $(10.2,1.84),(10.2$,

$\#$ 1.88), (10.4, 1.92), $(10.5,1.96),(10.6,2.0)))$

\#BB Model - Network B Parameters (Hysteretic Behavior) !VERY IMPORTANT The second constant HAS units!!! 1/(sec * MPa**m ) 
mdb. models ['Model-1']. materials ['BaseFilm'] . hyperelastic. Hysteresis (table $=(($

$2.0,0.5,4.5,-1.0), \quad))$

\section{\#\#\#\#\#\#\#\#\#\#\#\#\#\#\# \\ \# \\ SECTIONS \\ \#\#\#\#\#\#\#\#\#\#\#\#\#\#\#}

\#Create Section "Top Film"

mdb. models ['Model-1']. HomogeneousSolidSection( material='

TopFilm', name='TopFilm', thickness=None)

\#Create Section "RoD"

mdb. models [ 'Model-1']. Homogeneous SolidSection( material='Rod', name='Rod',

thickness=None)

\#Create Section "BaseFilm"

mdb. models ['Model-1']. HomogeneousSolidSection( material='

BaseFilm', name=

'BaseFilm', thickness=None)

\#Assign TopFilm Section

mdb. models ['Model-1']. parts ['Part-1']. Section Assignment ( offset $=0.0$,

offsetField=', , offset Type=MIDDLE_SURFACE, region=Region ( faces $=$ mdb. models [ 'Model-1'] . parts [ 'Part-1']. faces . getSequenceFromMask (

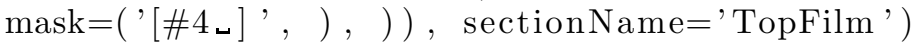

\#Assign BaseFilm Section

mdb. models ['Model-1']. parts ['Part-1']. SectionAssignment ( offset $=0.0$,

offsetField=', , offsetType=MIDDLE_SURFACE, region=Region ( faces $=$ mdb. models ['Model -1 '] . parts ['Part -1 '] . faces . getSequenceFromMask ( mask $\left.\left.\left.=(\text { '[\#1_ }]^{\prime},,\right), \quad\right)\right)$, sectionName='BaseFilm')

\#Assign Rod Section

mdb. models ['Model-1'] . parts ['Part-1']. SectionAssignment ( offset $=0.0$,

offsetField=', , offsetType=MIDDLE_SURFACE, region=Region ( faces $=$ mdb. models ['Model -1 '] . parts ['Part -1 '] ]. faces . getSequenceFromMask ( $\left.\left.\operatorname{mask}=\left({ }^{\prime}\left[\# 2_{\iota}\right] ',,\right), \quad\right)\right)$, sectionName $=$ 'Rod' $)$

\section{\#\#\#\#\#\#\#\#\#\#\#\#\#\#\#}

$\#$

MESH

\#\#\#\#\#\#\#\#\#\#\#\#\#\#\#

\#SEEDS

\#Seed Vertical Edges of the Top Film

mdb. models ['Model-1'] . parts ['Part-1'] . seedEdgeByNumber (edges= 
mdb. models ['Model-1'] . parts ['Part-1']. edges . getSequenceFromMask ( ('[\#140_ _ ] ,

), ) , number=S_VE_TF )

\#Seed Horizontal Edges of the Top Film and Base Film mdb. models ['Model-1'] . parts ['Part-1'] . seedEdgeBySize( edges= mdb. models ['Model-1'] . parts ['Part-1'] . edges . getSequenceFromMask (('[\#85 _ ]',

)$, \quad)$, size=S_HE_TF_BF )

\#Seed Vertical Edges of the Base Film mdb. models ['Model-1'] . parts ['Part -1 '] . seedEdgeBySize (edges= mdb. models ['Model-1'] . parts ['Part-1']. edges .

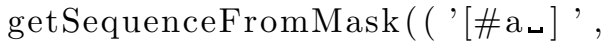

)$, \quad)$, size=S_HE_VE_BF )

\#Seed Ellipse Edges

mdb. models ['Model-1'] . parts ['Part-1'] . seedEdgeBySize( edges= mdb. models ['Model-1'] . parts ['Part-1']. edges . getSequenceFromMask(('[\#30_] ',

), ), size=S_E )

\section{\#MESH CONTROL}

\#Mesh Control@Top Film

mdb. models ['Model-1'] . parts ['Part-1']. setMeshControls ( elemShape=QUAD, regions=

mdb. models ['Model-1'] . parts ['Part-1']. faces . getSequenceFromMask(('[\#4_] ',

), ), technique $=$ STRUCTURED)

\#Mesh Controls on Mask \#3: Faces 0,1 (Base Film, Actuator) \# mdb. models ['Model-1']. parts ['Part-1']. setMeshControls ( allowMapped=True,

\# regions=mdb. models ['Model-1']. parts ['Part-1']. faces. getSequenceFromMask (

\# '[\#3]', ), ))

mdb. models ['Model-1']. parts ['Part-1']. setMeshControls ( alg orith $\mathrm{m}=$ MEDIAL_AXIS, elemShape=QUAD, regions= mdb. models ['Model-1'] . parts ['Part-1']. faces . getSequenceFromMask(('[\#3ـ]', )$, \quad)$

\#ELEMENT TYPES

\#Element Type CPE4R, CPE3@ Mask \#6: Faces 1,2 Top Film, Actuator

\#Plane Strain Elements, Reduced Integration

mdb. models ['Model-1']. parts ['Part-1']. setElementType (elem Types $=($ Elem Type (

elemCode $=$ CPEAR, elemLibrary $=$ STANDARD, secondOrder Accuracy $=$ OFF

hourglassControl=DEFAULT, distortionControl=DEFAULT), ElemType (

elemCode=CPE3, elemLibrary $=$ STANDARD, distortionControl= DEFAULT $)$ ), regions $=($ 
mdb. models ['Model-1']. parts ['Part-1']. faces . getSequenceFromMask ( ('[\#6_ $]$ ',

)$, \quad), \quad)$

\#Element Type CPE4RH, CPE3H @ Mask \#1: Face 0 (Base Film) \#Hybrid, Plane Strain Elements, Reduced Integration \#NOTE: Hybrid elements are needed for INCOMPRESSIBLE material mdb. models ['Model-1'] . parts ['Part-1'] . setElementType (elemTypes $=($ ElemType (

elemCode $=$ CPE4RH, elemLibrary $=$ STANDARD, secondOrderAccuracy $=$ $\mathrm{OFF}$,

hourglassControl=DEFAULT), ElemType( elemCode=CPE3H, elemLibrary $=$ STANDARD) ),

regions $=($ mdb. models ['Model -1 '] . parts ['Part -1 '] . faces . getSequenceFromMask (

,[\#1_]', ), ), ))

\section{\#GENERATE MESH}

\# \#Generate Mesh on Mask\#4:Face 2 (Top Film)

\# mdb. models ['Model-1']. parts ['Part-1']. generateMesh (regions= \# mdb. models ['Model-1']. parts ['Part-1']. faces. getSequenceFromMask (('[\#4]',

\# ), )

\# \#Generate Mesh on Mask\#2:Face 1 (Actuator)

\# mdb. models ['Model-1'].parts ['Part-1'].generateMesh(regions= \# mdb. models ['Model-1']. parts ['Part-1']. faces. getSequenceFromMask (('[\#1]', \# ), ))

\# \#Generate Mesh on Mask\#1:Face O (Base Film) \# mdb. models ['Model-1']. parts ['Part-1'].generateMesh (regions= \# mdb. models ['Model-1']. parts ['Part-1'].faces. getSequenceFromMask (('[\#1]',

\# ), ))

\#mdb. models ['Model-1']. parts ['Part-1']. deleteMesh ()

mdb. models ['Model-1']. parts ['Part-1'].

DatumPlaneByPrincipalPlane ( of f s et=EL_Y, principalPlane $=$ XZPLANE)

mdb. models ['Model-1'] . parts ['Part -1 '] .

DatumPlaneByPrincipalPlane ( off set $=0.0$, principalPlane $=$ YZPLANE)

mdb. models ['Model-1'] . parts ['Part-1'] .

PartitionFaceByDatumPlane (datumPlane $=$ mdb. models ['Model-1']. parts ['Part-1'] . datums [33], faces $=$ mdb. models ['Model-1']. parts ['Part-1']. faces . )$, \quad))$ getSequenceFromMask ( ('[\#7 _ ] ',

mdb. models ['Model-1'] . parts ['Part-1'] .

PartitionFaceByDatumPlane (datumPlane $=$ 
mdb. models ['Model-1'].parts ['Part-1'] . datums [32], faces $=$

mdb. models ['Model-1']. parts ['Part-1']. faces .

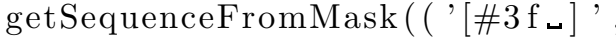
)$, \quad)$ )

mdb. models ['Model-1']. parts ['Part-1']. setMeshControls ( regions= mdb. models ['Model-1'] . parts ['Part-1'] . faces . getSequenceFromMask ( ('[\#210_ $]$ ',

), ), technique=STRUCTURED)

mdb. models ['Model-1'] . parts ['Part -1 '] . setMeshControls (regions= mdb. models ['Model-1']. parts ['Part-1']. faces . getSequenceFromMask(('[\#80 _ ] ', ), ), technique=STRUCTURED)

mdb. models ['Model-1']. parts ['Part-1'] . setMeshControls ( regions= mdb. models ['Model-1']. parts ['Part-1']. faces . getSequenceFromMask ( ('[\#40_ _ , ), ), technique=STRUCTURED)

mdb. models ['Model-1']. parts ['Part-1']. setMeshControls (regions= mdb. models ['Model-1'] . parts ['Part-1']. faces . getSequenceFromMask ( ('[\#1_ ] ', ), ), technique=STRUCTURED)

mdb. models ['Model-1'] . parts ['Part -1 '] . setMeshControls (regions= mdb. models ['Model-1']. parts ['Part-1']. faces . getSequenceFromMask ( ('[\#8 ] ', ), ), technique=STRUCTURED)

mdb. models ['Model-1']. parts ['Part-1']. setMeshControls (regions= mdb. models ['Model-1']. parts ['Part-1']. faces . getSequenceFromMask (('[\#100_ ] ', ), ), technique=STRUCTURED)

mdb. models ['Model-1']. parts ['Part-1']. setMeshControls (regions= mdb. models ['Model-1']. parts ['Part-1']. faces . getSequenceFromMask (('[\#20_ ]', ), ), technique=STRUCTURED)

mdb. models ['Model-1']. parts ['Part-1']. setMeshControls ( regions= mdb. models ['Model-1']. parts ['Part-1']. faces . getSequenceFromMask ( ('[\#2 _ ] ', ), ), technique=STRUCTURED)

mdb. models ['Model-1']. parts ['Part-1']. setMeshControls ( regions= mdb. models ['Model-1']. parts ['Part-1']. faces . getSequenceFromMask(('[\#4_ ] ', ), ), technique=STRUCTURED)

mdb. models ['Model-1'] $\cdot$ parts ['Part -1 '] . generateMesh(regions= mdb. models ['Model-1'] . parts ['Part-1']. faces . getSequenceFromMask ( ('[\#210_ ]', )$, \quad)$ )

mdb. models ['Model-1'] $\cdot$ parts ['Part -1 '] . generateMesh ( regions= mdb. models ['Model-1']. parts ['Part-1']. faces . getSequenceFromMask(('[\#126_ ] ', )$, \quad))$

mdb. models ['Model-1'] . parts ['Part -1 '] . generateMesh(regions= mdb. models ['Model-1']. parts ['Part-1']. faces . getSequenceFromMask ( ('[\#c9_ ]', )$, \quad)$ 
\#Mesh the base film using free meshing mdb. models ['Model-1']. parts ['Part-1'] . setMeshControls ( alg o r it $\mathrm{h} \mathrm{m}=\mathrm{ADVANCING \_ FRONT}$

, elemShape=QUAD, regions= mdb. models ['Model-1']. parts ['Part-1']. faces . getSequenceFromMask ( ('[\#c9 $\left.]_{\text {工 }}\right]$ ', ), ), technique=FREE)

mdb. models ['Model-1'] . parts ['Part-1'] . generateMesh (regions= mdb. models ['Model-1']. parts ['Part-1']. faces . getSequenceFromMask ( ('[\#80 $\left.]_{\text {U }}\right]^{\prime}$, )$, \quad)$ )

mdb. models ['Model-1'] . parts ['Part-1']. generateMesh ( regions= mdb. models ['Model-1']. parts ['Part-1']. faces . getSequenceFromMask ( ('[\#40_ ] ', )$, \quad)$ )

mdb. models ['Model-1']. parts ['Part-1']. generateMesh ( regions= mdb. models ['Model-1']. parts ['Part-1']. faces . )$, \quad)$ getSequenceFromMask ( ('[\#1_ ] ',

mdb. models ['Model-1'] . parts ['Part-1']. generateMesh (regions= mdb. models ['Model-1'] . parts ['Part-1']. faces .

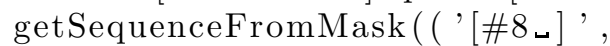
)$, \quad)$

mdb. models ['Model-1'] . rootAssembly . regenerate ()

\#\#\#\#\#\#\#\#\#\#\#\#\#

\#

SOLUTION

\#\#\#\#\#\#\#\#\#\#\#\#\#\#

\#Paste Post Processing Macro

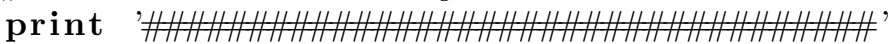

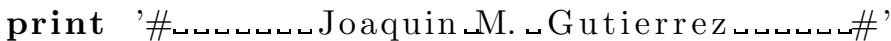

print '\#_West_ Virginia_University _-_2010 _\#'

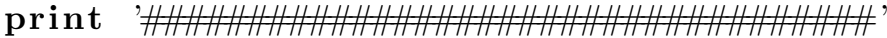

\#To make the Odb commands available to your script, you first need to import the odbAccess module using the following statements:

from odbAccess import *

from abaqusConstants import *

\#To make the material and section Odb commands available to your script, you also need to import the relevant module using the following statements:

from odbMaterial import *

from odbSection import *

\#Import Libraries

import datetime

import $\operatorname{csv}$ 
mdb. models ['Model-1']. StaticStep (description='Apply Load', initialInc=inInc, maxNumInc=int $(200 *$ numberCycles), name='OneCycle', nlgeom $=\mathrm{ON}$, noStop=OFF, previous='Initial', timeIncrementationMethod=FIXED, timePeriod $=$ analysisPeriod)

\# \#WARNING: Changed to Automatic Incrementation. There will no longer be frames at fixed incremental

time. The solver will choose.

mdb. models ['Model-1']. steps ['OneCycle']. setValues ( timeIncrementationMethod $=$ AUTOMATIC)

mdb. models ['Model-1']. steps ['OneCycle']. setValues ( initial Inc=

inInc, $\operatorname{maxInc}=$ inInc, $\operatorname{maxNumInc}=250, \operatorname{minInc}=$ in Inc / 1000.0)

\#Load-One Cycle

\#WARNING: check the stepnames

mdb. models ['Model-1']. SurfaceTraction (amplitude='Sine , createStepName=

'OneCycle', directionVector $=\left(\left(\begin{array}{lll}0.0, & 0.0,0.0\end{array}\right)\right.$, $(1.0,0.0,0.0))$,

distribution Type=UNIFORM, field=' , , follower= OFF, localCsys=None, magnitude=magnitudeSurfaceTraction , name=' TopLoad', region= mdb. models ['Model-1'] . rootAssembly. instances [' Part-1-1']. surfaces ['Ellipse-TopSurface'] , resultant $=\mathrm{ON}$, traction $=$ GENERAL)

mdb. models ['Model-1'] . Load (name='BottomLoad' , objectToCopy= mdb. models ['Model-1']. loads ['TopLoad']) mdb. models ['Model-1']. loads ['BottomLoad']. setValues ( magnitude $=-1.00 *$ magnitudeSurfaceTraction, region= mdb. models ['Model-1'] r root Assembly. instances [' Part-1-1']. surfaces ['Ellipse-BottomSurface '])

\#Regenerate Assembly mdb. models ['Model-1'] . rootAssembly .regenerate ()

\#Create Job

\# jobname=now. strftime ("\%Y-\%m-\%d-\%H_\%M_\%S")

\# mdb.Job(atTime=None, contactPrint=OFF, description $=$ ', echoPrint $=$ OFF, \# explicitPrecision $=$ SINGLE, getMemoryFromAnalysis $=$ True, historyPrint $=$ OFF,

\# memory $=50$, memoryUnits=PERCENTAGE, model=' Model-1', modelPrint=OFF, \# multiprocessing Mode=DEFAULT, name=jobname, nodalOutputPrecision $=$ SINGLE, 
\# numCpus=1, numDomains=1, parallelization MethodExplicit $=$ DOMAIN, queue=None,

\# scratch $=$ ', type $=$ ANALYSIS, userSubroutine $=$ ', waitHours $=0$, waitMinutes $=0$ )

\# Parallel Solving, Allocate More CPU and Memory jobname=now . strftime ( "\%Y_\%m-\%d-\%H_\%M_\%S" )

mdb. Job (atTime $=$ None, contactPrint $=$ OFF, description=, , , echoPrint $=\mathrm{OFF}$, explicitPrecision=SINGLE, getMemoryFromAnalysis=True, historyPrint= $\mathrm{OFF}$, memory $=85$, memoryUnits=PERCENTAGE, model $=$ ' Model-1', modelPrint $=$ OFF, multiprocessing Mode=THREADS, name=jobname , nodalOutputPrecision=SINGLE, numCpus $=4$, numDomains $=4$, parallelization MethodExplicit $=$ DOMAIN, queue $=$ None, scratch $=$ ', , type=ANALYSIS, userSubroutine $=$ ', , waitHours $=0$, waitMinutes $=0$ )

\#Define Output Requests for the Model mdb. models ['Model-1']. fieldOutputRequests ['F-Output-1

'] . setValues ( variables $=($

'IVOL' , 'LE', 's', 'U'))

\#Request Recoverable Strain Energy (SE) at the last increment for the PolymerMatrix Set

mdb. models ['Model-1']. HistoryOutputRequest ( createStepName=' OneCycle', frequency=LAST_INCREMENT, name='H-Output -2 ' , rebar= EXCLUDE, region= mdb. models ['Model-1'] . rootAssembly. instances ['Part $-1-1$ ']. sets ['PolymerMatrix'] , sectionPoints=DEFAULT, variables=('ALLSE', ) )

\#Submit Job

job $=$ mdb. jobs $[$ jobname $]$

job.submit ( consistencyChecking $=\mathrm{OFF}$ )

job.waitFor Completion ()

print" Frequency Solved"

\#Begin the Post-Processing

\#Open The Output Data Base of the Current Job

odb $=$ openOdb $($ path=jobname+' . odb' $)$

myAssembly = odb.rootAssembly 


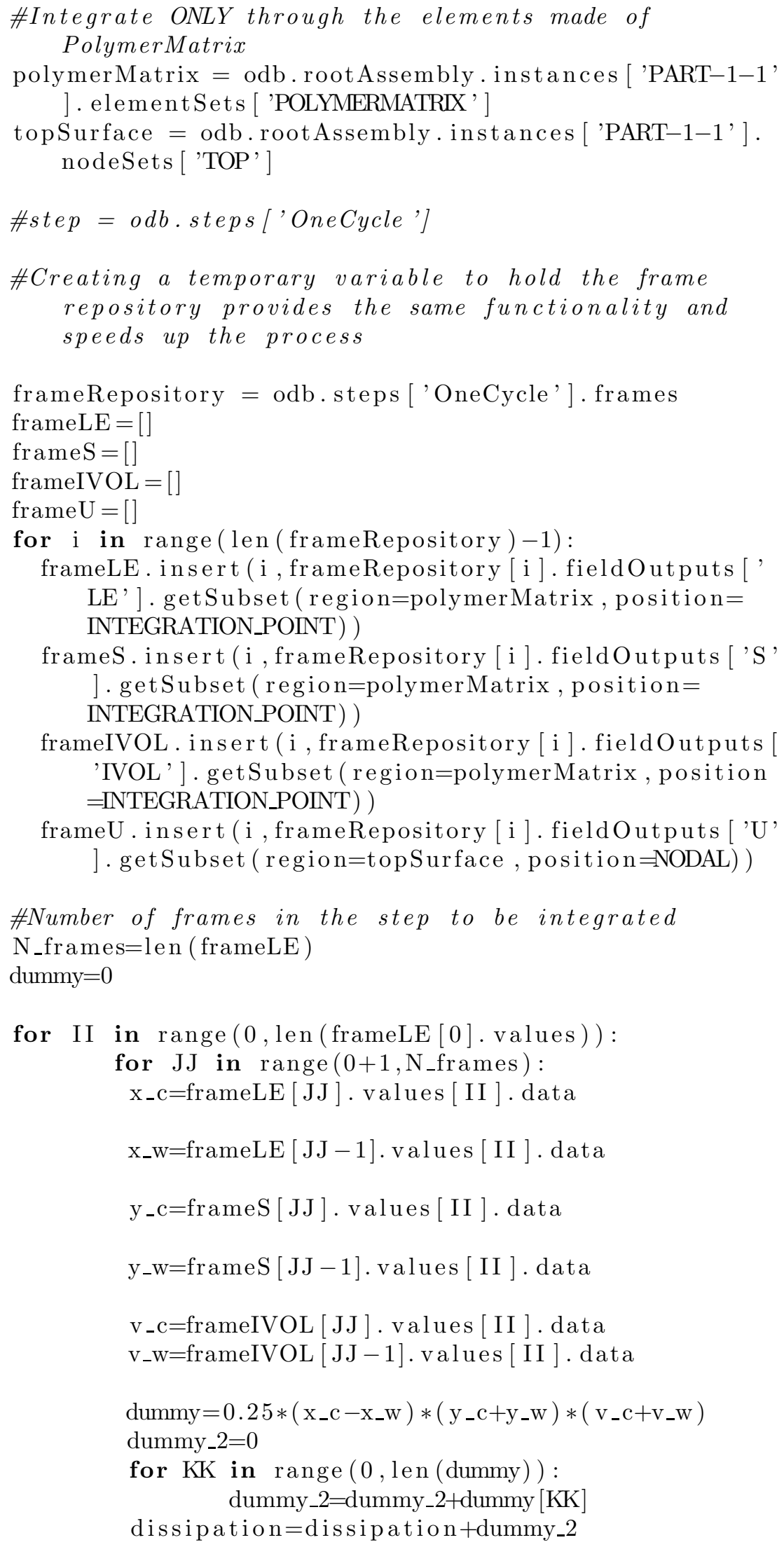




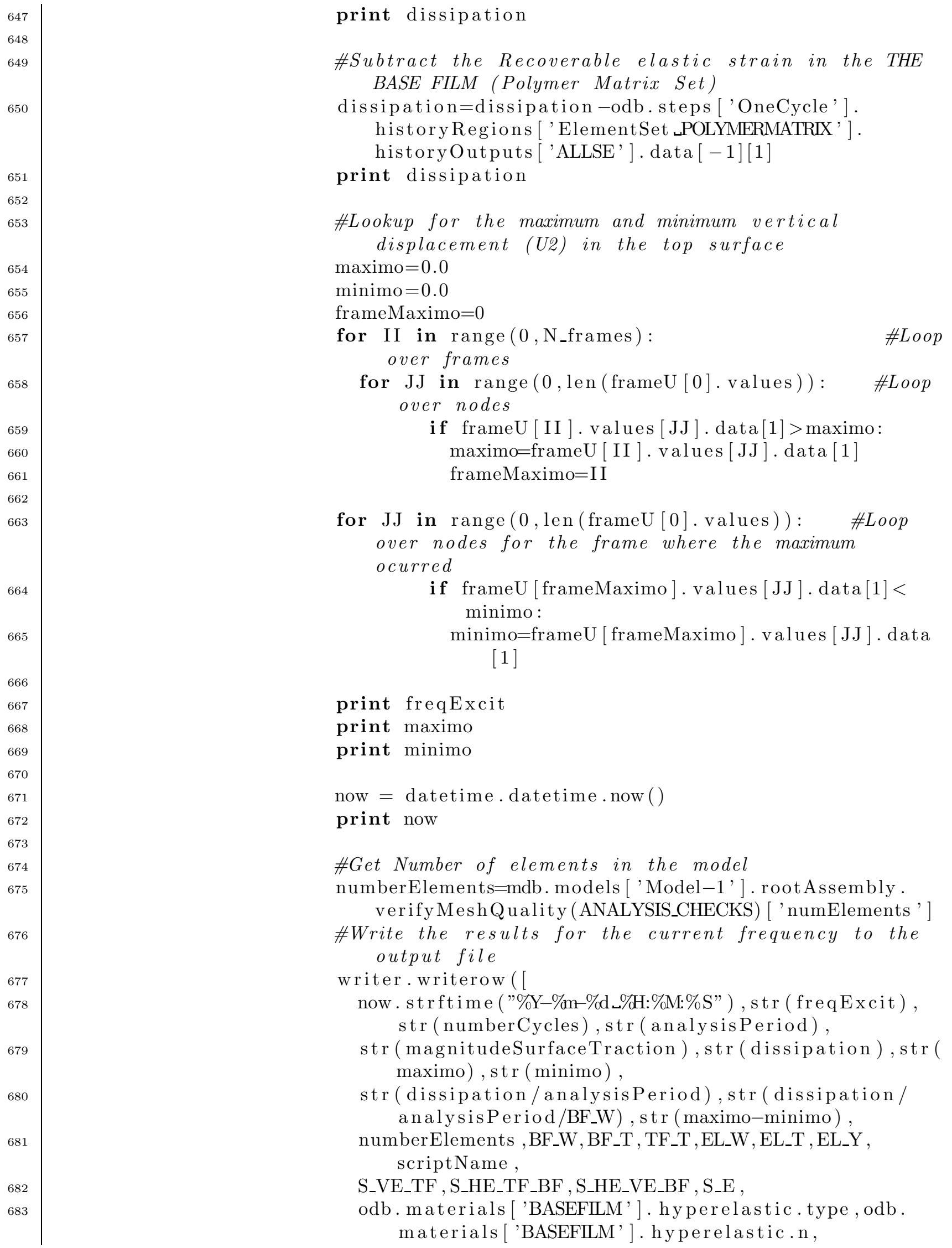




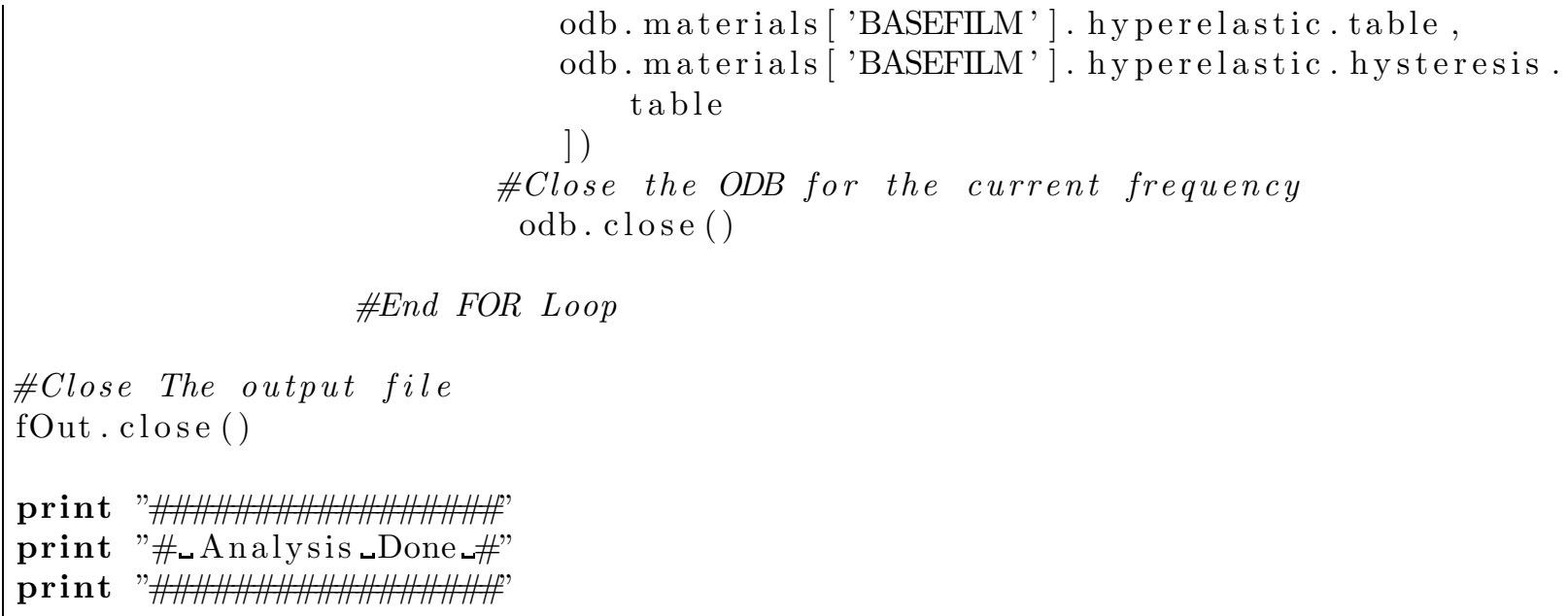

\section{E.3 Three-Dimensional Model}

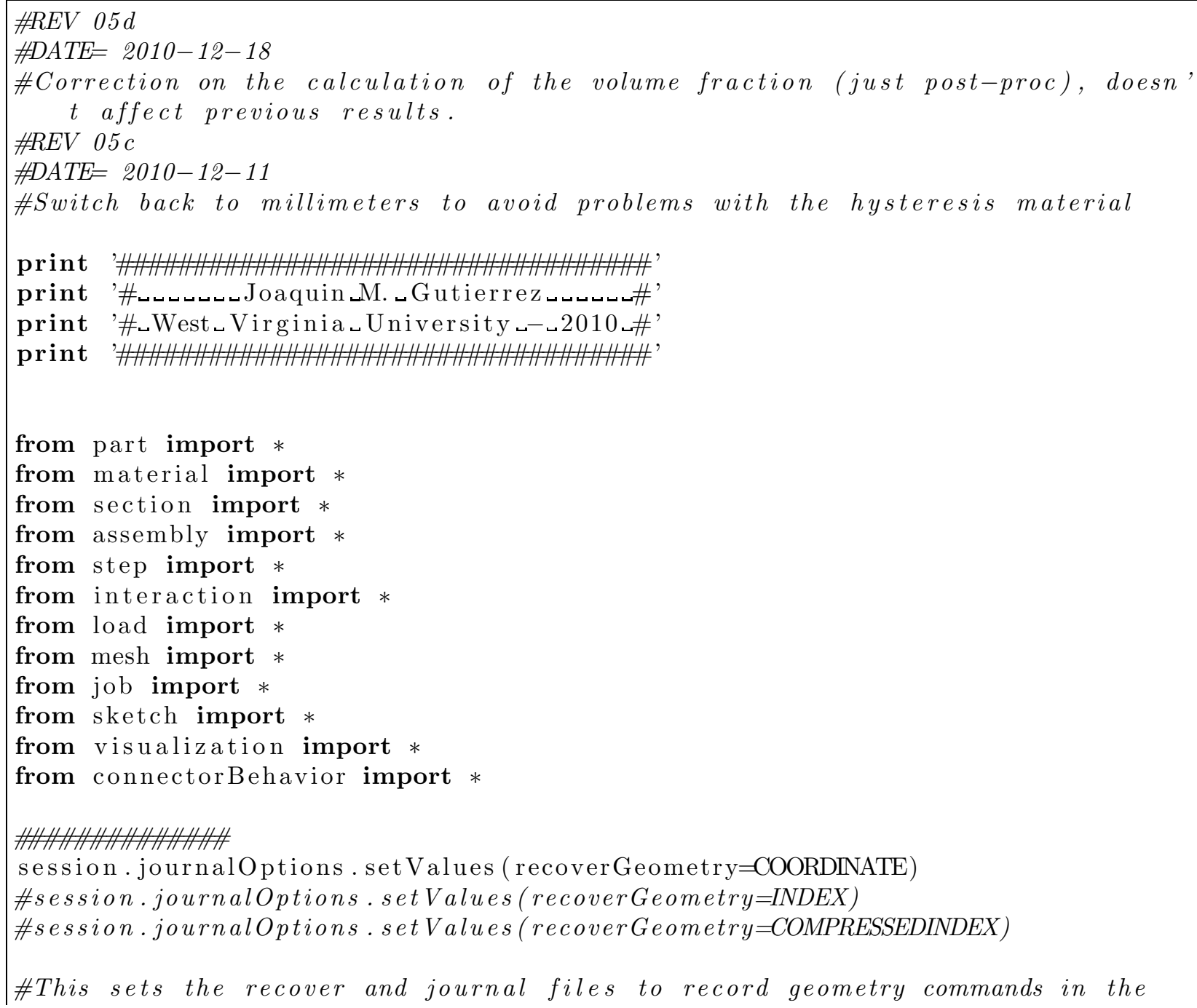


\#form of coordinates instead of geometric indices. This step is very helpful \#if any type of parameterization will be used with the script.

\#\#\#\#\#\#\#\#\#\#\#

\#\#\#\#\#\#\#\#\#\#\#\#\#\#

\#Variables

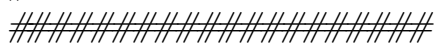

\#S cript Name (It is important to keep it updated, for proper documentation of \#the results)

scriptName=' 3DMODEL05d.py' \#!!!! KEEP ME UPDATED!!!!

\#Geometry (USE .0 to make these figures FLOATS and not INTEGERS!!!!)

BF_W $=60.0 \mathrm{E}-3 * 5.0 \quad$ \#Base Film Width [mm]

BF_T $=30.0 \mathrm{E}-3 \quad$ \#Base Film Thickness [mm]

$\mathrm{BF} \_\mathrm{D}=5.0 \mathrm{E}-3 * 2.5$ \#Base Film Depth [mm] (In the extrusion direction)

TF_T $=0.03 \mathrm{E}-3 \quad$ \#Top Film Thickness [mm]

EL_W=60.0E-3 \#Ellipsoid Major Diameter [mm]

EL_T $=10.0 \mathrm{E}-3 \quad$ \#Ellipsoid Minor Diameter [mm]

\#Mesh Seeds (Input INTEGERS for Number of Seeds and FLOATS for Element Sizes !!!)

\# \#These seeds are to be used in the 6.10 Student Edition (1000 nodes limit)

$\# S_{-} D=2.0$

\# $S_{-} T=2.0$

\#Element size along the DEPTH of the RVE

\# $S_{-} T F=1$

\#Element size along the THICKNESS of the BF

$\# S_{-} W_{-} C=2.0$

$\# S_{-} W_{-} O_{-}$min $=2.0$ BIASED)

\# S_W_O_max=10.0 \#Max Element Size along the OUTER WIDTH of the RVE

\# $S_{-} E=2.0$

\# $S_{-} E_{-} d e v=0.1$ \#Element size along the ELLIPSE the ELLIPSE EDGES \#Deviator factor for curvature control allong

$\mathrm{S} \_\mathrm{D}=1.17857 \mathrm{E}-3 \quad$ \#2.0E-3

\#Element size along the DEPTH of the RVE

$\mathrm{S} \_\mathrm{T}=2.0 \mathrm{E}-3$ the $B F$

$\mathrm{S} \_\mathrm{TF}=1$ THICKNESS of the TF (USE INTEGER!!!) \#Element size along the THICKNESS of

S_W_C $=1.0 \mathrm{E}-3$ of the RVE

S_W_O_min $=1.0 \mathrm{E}-3$ WIDTH of the RVE (BIASED)

S_W_O_max $=20.0 \mathrm{E}-3 \quad$ \#Max Element Size along the OUTER WIDTH of the RVE

$\mathrm{S} \_\mathrm{E}=1.0 \mathrm{E}-3$ ELLIPSE

\#Element size along the

S_E_dev $=0.1$ curvature control allong the ELLIPSE EDGES

\#Deviator factor for

\#Definie the magnitude of the surface traction (load) to be applied (MPa)

magnitudeSurfaceTraction $=10.00$

\#Excitation Frequency

freqExit $=200.00$ 


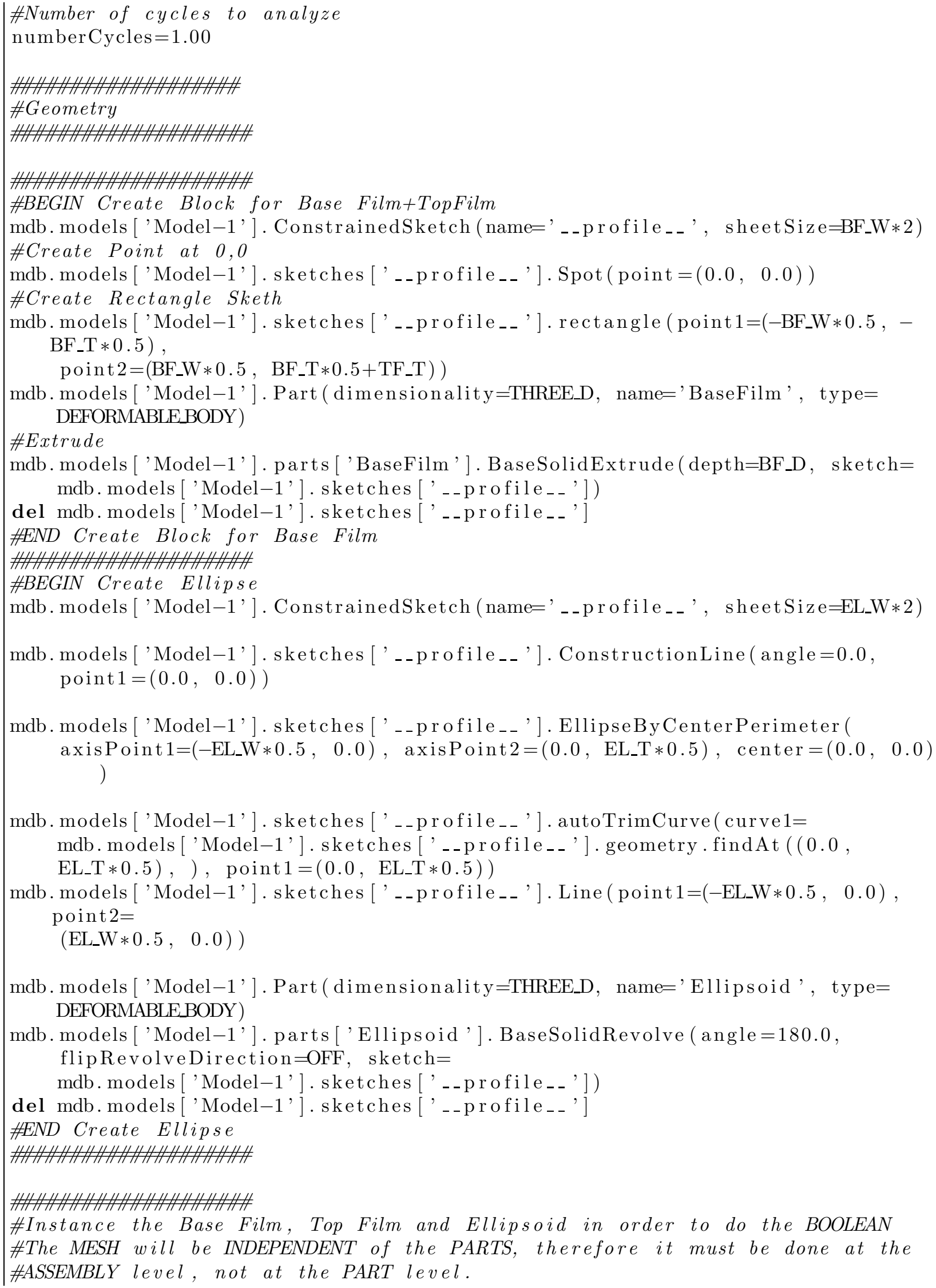


mdb. models ['Model-1'] . root Assembly . DatumCsysByDefault (CARTESIAN)

mdb. models ['Model-1'] rootAssembly. Instance( dependent=OFF, name='BaseFilm -1 ', part $=$ mdb. models ['Model -1 '] . parts ['BaseFilm'])

mdb. models ['Model-1'] rootAssembly. Instance (dependent=OFF, name=' Ellipsoid -1 ', part $=$ mdb. models ['Model-1']. parts ['Ellipsoid'])

mdb. models ['Model-1'] . root Assembly . InstanceFromBooleanMerge (domain=GEOMETRY, instances $=($ mdb. models ['Model -1 '] . rootAssembly . instances ['BaseFilm -1 '], mdb. models ['Model-1']. rootAssembly. instances ['Ellipsoid -1']), keepIntersections $=\mathrm{ON}$, name='RVE', originalInstances $=$ SUPPRESS )

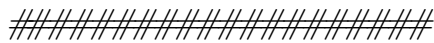

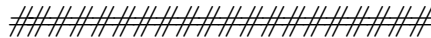

\#BEGIN Create Datum Planes and do Partitions on the RVE

mdb. models ['Model-1'] . parts [ 'RVE'] . DatumPlaneByPrincipalPlane( offset $=0.0$, principalPlane $=$ XZPLANE)

mdb. models ['Model-1']. parts [ 'RVE'] . DatumPlaneByPrincipalPlane( offset $=0.0$, principalPlane $=$ YZPLANE)

mdb. models ['Model-1'] . parts [ 'RVE'] . DatumPlaneByPrincipalPlane( offset=EL_W $* 0.75$, principalPlane $=$ YZPLANE)

mdb. models ['Model-1'] . parts ['RVE' ]. DatumPlaneByPrincipalPlane ( of f set=-EL_W $* 0.75$, principalPlan $\mathrm{e}=$ YZPLANE)

mdb. models [ 'Model-1'] . parts ['RVE'] . DatumPlaneByPrincipalPlane( offset=BF_T $* 0.5$, principalPlane=XZPLANE)

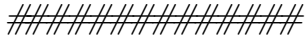

\#S witch to COMPRESSEDINEX notation

session . journalOptions . setValues ( recoverGeometry=COMPRESSEDINDEX) \#\#\#\#\#\#\#\#\#\#\#

\#Partitions using COMPRESSED INDEXES

mdb. models ['Model-1'] . parts [ 'RVE']. PartitionCellBy DatumPlane ( cells= mdb. models ['Model-1'] . parts ['RVE'] . cells .getSequenceFromMask (('[\#3_] ', ), ) , datumPlane $=$ mdb. models ['Model-1'] . parts ['RVE']. datums [3])

mdb. models ['Model-1'] . parts [ 'RVE']. Partition CellByDatumPlane ( cells= mdb. models ['Model-1'] . parts ['RVE'] . cells .getSequenceFromMask (('[\#f_]', ), , datumPlane=mdb. models ['Model -1 '] . parts ['RVE']. datums [2]) mdb. models ['Model-1'] . parts ['RVE'] . Partition CellBy DatumPlane ( cells= mdb. models ['Model-1'] . parts ['RVE'] . cells .getSequenceFromMask (('[\#88_]', ), ), datumPlane $=$ mdb. models ['Model-1']. parts ['RVE']. datums [6])

mdb. models ['Model-1'] . parts ['RVE']. Partition CellBy DatumPlane ( cells= mdb. models ['Model-1'] . parts [ 'RVE'] . cells . getSequenceFromMask (('[\#62 _ ]', ), ), datumPlane $=$ mdb. models ['Model-1']. parts ['RVE']. datums [5])

mdb. models ['Model-1'] . parts [ 'RVE']. Partition CellByDatumPlane ( cells= mdb. models ['Model-1'] . parts [ 'RVE'] . cells .getSequenceFromMask(('[\#1028_] ', ), ), datumPlane=mdb. models ['Model-1'] . parts ['RVE']. datums [4]) 


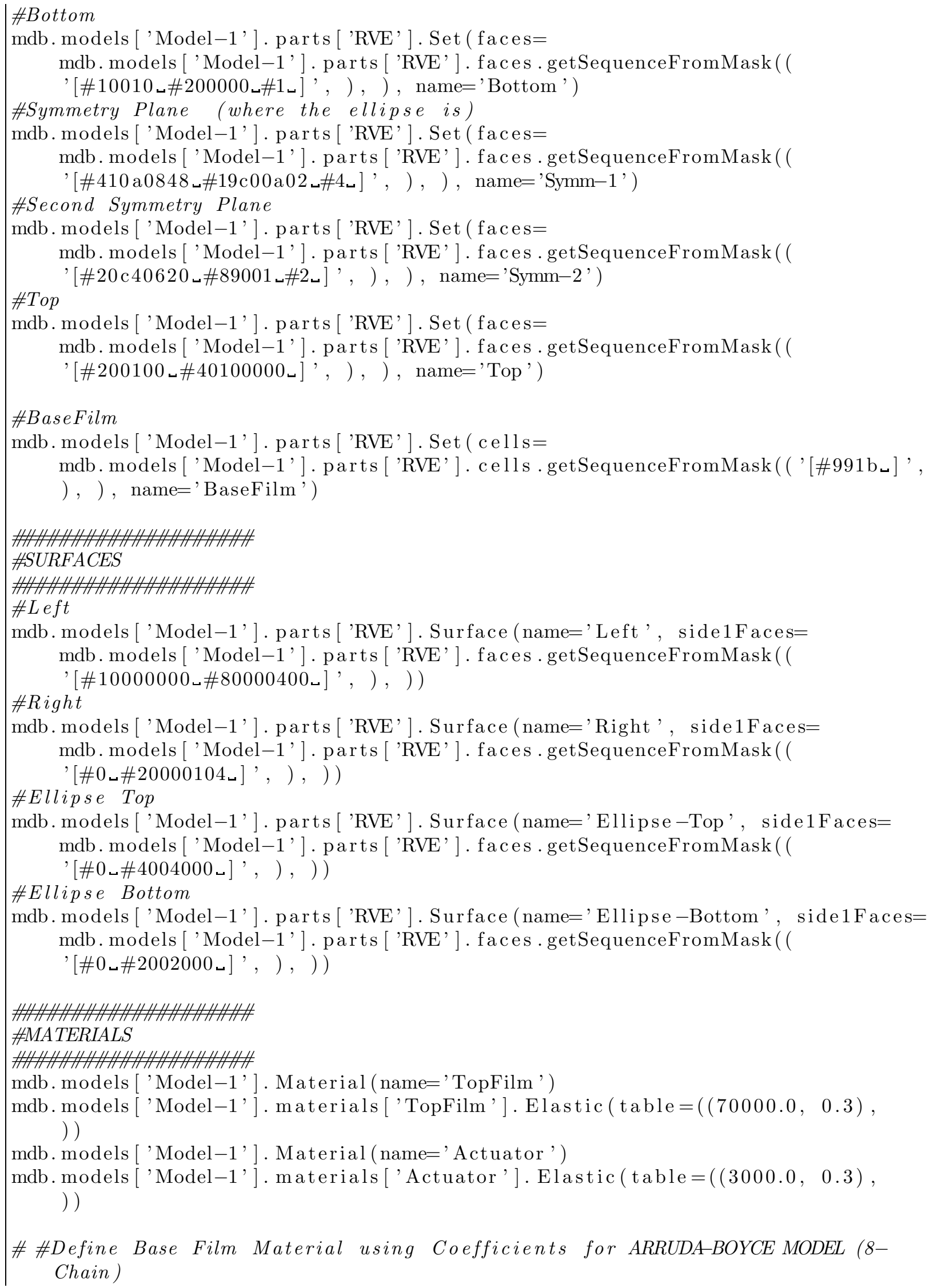


231 \# mdb. models ['Model-1']. Material (name='BaseFilm')

\# mdb. models ['Model-1']. materials ['BaseFilm']. hyperelastic. setValues (table =(( \# 4680320.25, 7083.00147, 0.0), ))

\# \#BB Model - Network B Parameters (Hysteretic Behavior)

\# mdb. models ['Model-1']. materials ['BaseFilm']. hyperelastic. Hysteresis (table $=(($ \# 2.0, 0.5, 4.5, - -1.0), ))

\#Define Base Film Material using Coefficients for ARRUDA-BOYCE MODEL (8-Chain) \#Hytrel 4056 (TPE)

mdb. models ['Model-1']. Material (name='BaseFilm')

\# mdb. models ['Model-1']. materials ['BaseFilm']. Hyperelastic ( table $=(($ \# 4680320.25, 7083.00147, 0.0), ), testData=OFF, type=ARRUDA_BOYCE, \# volumetric Response=VOLUMETRIC_DATA)

\#Units in $N, m m$

mdb. models ['Model-1'] . materials ['BaseFilm']. Hyperelastic ( table $=(($ $4.68032146,5311.53,0.0)$, ), testData=OFF, type=ARRUDA_BOYCE, volumetricResponse=VOLUMETRICDATA)

\# mdb. models ['Model-1']. materials ['BaseFilm ']. hyperelastic. UniaxialTestData( \# table $=((2.83,0.0471),(4.29,0.0833),(5.5,0.127),(6.2,0.163)$, (6.83, $\# 0.207),(7.21,0.243),(7.55,0.286),(7.74,0.323),(7.92,0.366)$, $(8.02$, $\#$ 0.402) $,(8.09,0.446),(8.15,0.482),(8.18,0.525),(8.2,0.562)$, $(8.23$,

$\# 0.605),(8.25,0.641),(8.29,0.685),(8.32,0.721),(8.36,0.765)$, $(8.39$

$\# 0.801),(8.44,0.844),(8.48,0.881),(8.53,0.924),(8.57,0.96)$, $(8.62$,

$\# 1.0),(8.69,1.05),(8.73,1.08),(8.79,1.13),(8.84,1.16),(8.91$, 1.21)

$\#(8.96,1.24),(9.03,1.29),(9.09,1.32),(9.17,1.37),(9.22,1.4)$, (9.3,

$\# 1.45),(9.37,1.48),(9.45,1.53),(9.52,1.56),(9.6,1.61),(9.68$, $1.64)$

$\#(9.78,1.68),(9.86,1.72),(10.0,1.76),(10.1,1.8),(10.2,1.84)$, (10.2,

$\# 1.88),(10.4,1.92),(10.5,1.96),(10.6,2.0)))$

\#BB Model - Network B Parameters (Hysteretic Behavior) !VERY IMPORTANT The second constant HAS units!!! 1/( sec * MPa**m)

mdb. models ['Model-1']. materials ['BaseFilm'] . hyperelastic. Hysteresis ( table $=(($ $2.0,0.5,4.5,-1.0), \quad))$

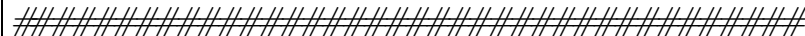

$\#$

\section{SECTIONS}

mdb. models ['Model-1']. HomogeneousSolidSection (material='TopFilm', name= 'TopFilm', thickness=None)

mdb. models ['Model-1']. HomogeneousSolidSection(material='Actuator', name= 'Actuator', thickness=None)

mdb. models ['Model-1']. HomogeneousSolidSection(material='BaseFilm', name= 'BaseFilm', thickness=None) 
\# mdb. models ['Model-1'].parts ['RVE']. SectionAssignment(offset=0.0, offsetField $=$, \# , offset Type=MIDDLE_SURFACE, region=Region ( \# cells=mdb. models ['Model-1']. parts ['RVE']. c ells.getSequenceFromMask (mask $=($ \# '[\#e4 ]', ), )), sectionName='TopFilm', thicknessAssignment=FROM_SECTION )

mdb. models ['Model-1'] . parts ['RVE']. SectionAssignment ( offset =0.0, offsetField=' , offset Type=MIDDLE_SURFACE, region=Region ( c ells $=$ mdb. models ['Model-1'] . parts ['RVE'] . c ells . getSequenceFromMask (mask=( '[\# $\left.4_{\mathbf{L}}\right]$ ', , ), )), sectionName='TopFilm')

\# mdb. models ['Model-1']. parts ['RVE']. SectionAssignment(offset=0.0, offsetField $=$,

\# , offsetType=MIDDLE_SURFACE, region=Region (

\# cells=mdb. models ['Model-1']. parts ['RVE']. cells.getSequenceFromMask (mask $=($

\# '[\#6600]', ), )), sectionName='Actuator', thicknessAssignment= \# FROM_SECTION)

mdb. models ['Model-1']. parts ['RVE']. SectionAssignment ( offset $=0.0$, offsetField=' , offset Type=MIDDLE_SURFACE, region=Region ( c ells $=$ mdb. models ['Model-1'] . parts [ 'RVE'] . cells . getSequenceFromMask ( mask $=($ '[\#6600_ ]', ), )), sectionName='Actuator')

\# mdb. models ['Model-1']. parts ['RVE']. SectionAssignment(offset=0.0, offsetField $=$,

\# , offsetType=MIDDLE_SURFACE, region=Region (

\# cells=mdb. models ['Model-1']. parts ['RVE']. c ells.getSequenceFromMask (mask $=($

\# '[\#991b]', ), )), sectionName='BaseFilm', thicknessAssignment= \# FROM_SECTION)

mdb. models ['Model-1']. parts [ 'RVE']. SectionAssignment ( offset $=0.0$, offsetField=' , offset Type=MIDDLE_SURFACE, region=Region ( c ells $=$ mdb. models ['Model-1'] · parts ['RVE'] . cells . getSequenceFromMask (mask=( '[\#991bь]', ), )), sectionName='BaseFilm')

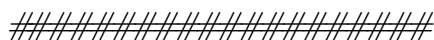

\#MESH Module

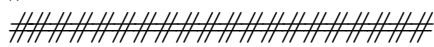

\#Make the RVE Instance as Independent

mdb. models ['Model-1'] . root Assembly . makeIndependent ( instances $=($ mdb. models ['Model-1']. rootAssembly. instances ['RVE-1'], ))

\section{\#SEEDS}

\#Using CompressedIndexes, won't work if the geometry is changed its entities, not its dimensions 
\#Biased SEEDS along the WIDTH

mdb. models ['Model-1'] . rootAssembly . seedEdgeByBias ( bias Method=SINGLE, constraint $=$ FINER, end 1 Edges $=$ mdb. models ['Model-1'] rootAssembly . instances ['RVE-1'] . edges . getSequenceFromMask (

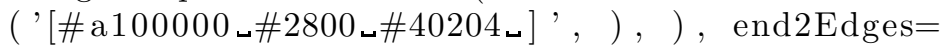
mdb. models ['Model-1'] rootAssembly . instances ['RVE-1'] . edges . getSequenceFromMask (

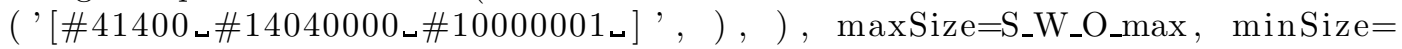
S_W_O_min)

mdb. models ['Model-1'] . rootAssembly . Set ( edges= mdb. models ['Model-1'] r root Assembly . instances [ 'RVE-1'] edges . getSequenceFromMask

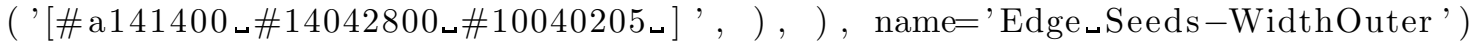

\#Seed the CENTRAL WIDTH

mdb. models ['Model-1'] . rootAssembly . seedEdgeBySize ( constraint=FINER, deviationFactor $=0.1$, edges $=$ mdb. models ['Model-1'] r rootAssembly . instances ['RVE-1'] . edges . getSequenceFromMask( ('[\#5141a000_\#a0920000_\#40000400_]'，），），， s ize=S_W_C)

mdb. models ['Model-1'] . rootAssembly . Set ( edges= mdb. models ['Model-1'] r rootAssembly . instances ['RVE-1'] . edges . getSequenceFromMask (

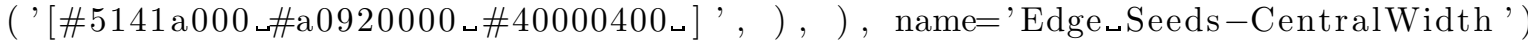

\#Seed along the DEPTH of the RVE

mdb. models ['Model-1'] . rootAssembly . seedEdgeBySize ( constraint=FINER, deviationFactor $=0.1$, edges $=$ mdb. models ['Model-1'] rootAssembly . instances ['RVE-1'] . edges . getSequenceFromMask (

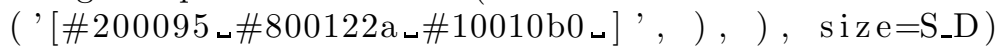

mdb. models ['Model-1'] $\operatorname{rootAssembly} \cdot$ Set ( edges= mdb. models ['Model-1'] r rootAssembly . instances ['RVE-1'] . edges . getSequenceFromMask

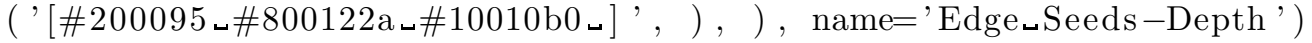

\#Seed the BASE FILM THICKNESS

mdb. models ['Model-1'] . rootAssembly . seedEdgeBySize ( constraint=FINER, deviationFactor $=0.1$, edges $=$ mdb. models ['Model-1'] . root Assembly . instances ['RVE-1']. edges . getSequenceFromMask (

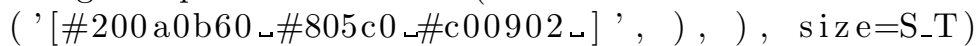

mdb. models ['Model-1'] $\operatorname{rootAssembly} \cdot$ Set ( edges= mdb. models ['Model-1'] rootAssembly . instances ['RVE-1'] . edges . getSequenceFromMask (

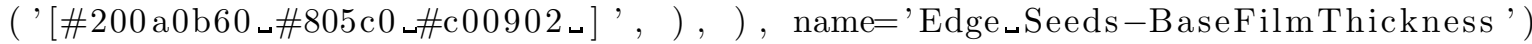

\#Seed the Ellipse

mdb. models ['Model-1'] . rootAssembly . seedEdgeBySize ( constraint=FINER, deviationFactor=S_E_dev, edges= 
374

mdb. models ['Model-1']. rootAssembly. instances ['RVE-1']. edges . getSequenceFromMask (

('[\#0_\#2408000_\#2e1bc000_]', , ), ), size=S_E )

mdb. models ['Model-1'] . rootAssembly . Set ( edges=

mdb. models ['Model-1'] . rootAssembly. instances [ 'RVE-1'] . edges . getSequenceFromMask (

('[\#0ヶ\#2408000ヶ\#2e1bc000ヶ]', , ), ), name='EdgeヶSeeds-Ellipse')

\#SEED the vertical edges (along the thickness) of the TOP FILM

mdb. models ['Model-1'] . rootAssembly. seedEdgeByNumber ( constraint=FINER, edges= mdb. models ['Model-1'] . root Assembly. instances ['RVE-1'] . edges . getSequenceFromMask (

('[\#8400000a_\#40000014_\#200048_]', , ), ), number=S_TF )

mdb. models [ 'Model-1'] . rootAssembly . Set ( edges=

mdb. models ['Model-1']. root Assembly. instances ['RVE-1']. edges . getSequenceFromMask (

('[\#8400000a_\#40000014॰\#200048_]', , ) , ) , name=

'Edge_Seeds-TopFilmThickness' )

\#Change the Element Type for the BaseFilm to Hybrid Formulation \#This needed in order to use incompressible Hyperlastic Material Model \#(C3D8RH): (C) ontinuum (3D) (8) Node (R) educed Integration (H)ybrid mdb. models ['Model-1'] . root Assembly . setElementType (elemTypes $=$ (ElemType ( elem Code $=$ C3D8RH, elemLibrary $=$ STANDARD, second OrderAccuracy $=$ OFF, kinematicSplit=AVERAGESTRAIN, hourglassControl=DEFAULT), ElemType( elemCode $=\mathrm{C} 3 \mathrm{D} 6 \mathrm{H}$, elemLibrary $=$ STANDARD) , ElemType (elemCode $=\mathrm{C} 3 \mathrm{D} 4 \mathrm{H}$, elemLibrary $=$ STANDARD) ) , regions $=($ mdb. models ['Model-1'] . root Assembly . instances ['RVE-1'] . cells . getSequenceFromMask ( $\left.\left.\left.\left({ }^{\prime}\left[\# 991 b_{\mathbf{\iota}}\right] ',,\right), \quad\right), \quad\right)\right)$

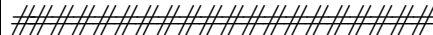

\#MESH the RVE (@INSTANCE level)

mdb. models ['Model-1'] . rootAssembly.generateMesh ( regions $=($ mdb. models ['Model-1']. root Assembly. instances ['RVE-1'], ))

\section{\#\#\#\#\#\#\#\#\#\#\#\#\#\#\#\#\# \#\#和}

$\# B C$ 's

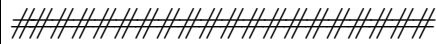

\#Bottom (Glued $\rightarrow U X=U Y=U Z=0$ )

mdb. models ['Model-1']. DisplacementBC ( a mplitude=UNSET, createStepName=' In itial' 'distribution Type=UNIFORM, field Name=', , localCsys=None, name='Bottom', region=mdb. models ['Model-1'] . rootAssembly. instances ['RVE-1'] . sets ['Bottom '

] , u1=SET, u2=SET, u3=SET, ur $1=$ UNSET, ur $2=$ UNSET, ur $3=$ UNSET)

\#Symm-1 ZSYMM (U3=UR1=UR2=0)

mdb. models ['Model-1'] .ZsymmBC( createStepName=' Initial', name='Symm-1', region= mdb. models ['Model-1'] . root Assembly . instances ['RVE-1']. sets ['Symm-1'])

\#Symm-2 ZSYMM (U3=UR1=UR2=0)

mdb. models ['Model-1'] .ZsymmBC( createStepName=' Initial', name='Symm-2', region= mdb. models ['Model-1'] . rootAssembly.instances ['RVE-1'] . sets ['Symm-2']) 


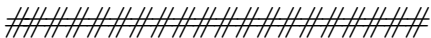

\#Periodic Boundary Conditions $\longrightarrow$ Constrains $\rightarrow$ Tie

\#WARNING 1: It is important to set the offset value correctly! (Base Film Width: BF_W)

\#WARNING 2: SURFACE_TO_SURFACE might be a feature new to ABAQUS 6.10

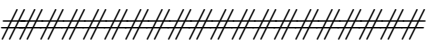

mdb. models ['Model-1'] . Tie ( adjust $=$ OFF, constraintEnfor cement=SURFACE_TO_SURFACE

master $=$

mdb. models ['Model-1'] . rootAssembly. instances ['RVE-1'] . surfaces ['Left'] , name='PBC', positionTolerance=BF_W $* 1.10$, positionToleranceMethod=SPECIFIED slave $=$ mdb. models ['Model-1'] . rootAssembly. instances ['RVE-1'] . surfaces ['Right'], thickness=ON, tieRotations $=\mathrm{OFF}$ )

\section{\#\#\#\#\#\#\#\#\#\#\#\#\#\#\#\#}

$\# L O A D$

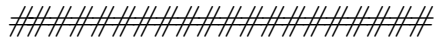

period Excit $=1.00 /$ freqExcit

\#Excitation Period \#Length of the Analysis

analysisPeriod $=$ periodExcit $*$ numberCycte angularFreqExcit $=2.00 * 3.1416 *$ freqExcit \#Angular Frequency of the Excitation inInc=periodExcit / $(120.00)$ \#Time Step Size

\#Create Amplitude

mdb. models ['Model-1'] . Periodic Amplitude $\left(a_{-} 0=0.0\right.$, data $=((0.0,1.0)$,$) ,$ frequency=angularFreqExcit, name='Sine', start $=0.0$, timeSpan=STEP)

\#Create Step 'SinusoidalTorque' to Apply Load

mdb. models ['Model-1'] . StaticStep (description='Apply Sinusoidal_Load', initial In $\mathrm{c}=$ in Inc , maxNumInc $=\operatorname{int}(200 *$ numberCycles $)$, name='SinusoidalTorque', nlgeom $=\mathrm{ON}$, noStop $=$ OFF, previous='Initial', timeIncrementationMethod=FIXED, timePeriod=analysisPeriod)

\#Apply Surface Traction on the Top Half of the Ellipsoid (Positive in the $X$ direction)

mdb. models ['Model-1']. SurfaceTraction (amplitude='Sine', createStepName= 'SinusoidalTorque', directionVector $=((0.0,0.0,0.0),(1.0,0.0,0.0))$, distributionType=UNIFORM, field=', , follower $=$ OFF, localCsys=None, magnitude=magnitudeSurfaceTraction, name='Ellipse-Top', region= mdb. models ['Model-1'] . rootAssembly.instances ['RVE-1'] . surfaces ['EllipseTop'] , resultant $=$ ON, traction=GENERAL)

\#Apply Surface Traction on the Bottom Half of the Ellipsoid (Negative in the $X$ direction)

mdb. models ['Model-1']. SurfaceTraction (amplitude='Sine', createStepName $=$ 'SinusoidalTorque', directionVector $=((0.0,0.0,0.0),(1.0,0.0,0.0))$, distributionType=UNIFORM, field=', , follower $=$ OFF, localCsys=None, magnitude=-magnitudeSurfaceTraction, name='Ellipse-Bottom', region= mdb. models ['Model-1'] root Assembly. instances ['RVE-1'] . surfaces ['EllipseBottom'] , resultant $=\mathrm{ON}$, traction $=$ GENERAL) 
\#Define Output Requests for the Model

mdb. models ['Model-1']. fieldOutputRequests ['F-Output-1'] . setValues (variables =( 'IVOL', 'LE', , 'S', 'U'))

\#Request Recoverable Strain Energy (SE) at the last increment for the BaseFilm Set

mdb. models ['Model-1']. HistoryOutputRequest ( createStepName='SinusoidalTorque', frequency=LAST_INCREMENT, name='H-Output-2', rebar=EXCLUDE, region= mdb. models ['Model-1'] root Assembly. instances ['RVE-1'] . sets ['BaseFilm' ], sectionPoints=DEFAULT, variables $=\left(\right.$ 'ALLSE' $\left.\left.^{\prime}, \quad\right)\right)$

\#Create Job

mdb. Job(atTime=None, contactPrint=OFF, description=', , echoPrint=OFF, explicitPrecision=SINGLE, getMemoryFromAnalysis=True, historyPrint=OFF, memory $=90$, memoryUnits=PERCENTAGE, model='Model-1', modelPrint=OFF, name= 'Job-1', nodalOutputPrecision=SINGLE, queue=None, scratch=' ', type= ANALYSIS , userSubroutine $=$, , , waitHours $=0$, waitMinutes $=0$ ) \#Submit

mdb.jobs ['Job-1']. submit ()

\#Wait for finish

mdb.jobs ['Job-1'] . waitFor Completion()

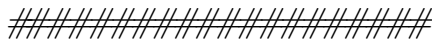

\#Post-Processing

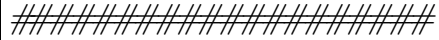

\#Reset Dissipation to Zero at the begining of each loop

dissipation $=0.0$

\#Begin the Post-Processing

\#Open The Output Data Base of the Current Job

$\mathrm{odb}=\operatorname{openOdb}\left(\right.$ path $=$ 'Job $\left.-1 . \mathrm{odb}{ }^{\prime}\right)$

myAssembly $=$ odb.rootAssembly

\#Integrate ONLY through the elements made of PolymerMatrix

BASEFILM $=$ odb.rootAssembly. instances [ 'RVE-1'] . elementSets ['BASEFILM']

$\mathrm{TOP}=$ odb.rootAssembly. instances [ RVE-1']. nodeSets [ 'TOP']

\#Creating a temporary variable to hold the frame repository provides the same functionality and speeds up the process

frameRepository $=$ odb.steps ['SinusoidalTorque']. frames

frameLE $=[]$

frameS $=[]$

frameIVOL $=[]$

frame $\mathrm{U}=[]$

for $\mathrm{i}$ in range(len(frameRepository)-1): 


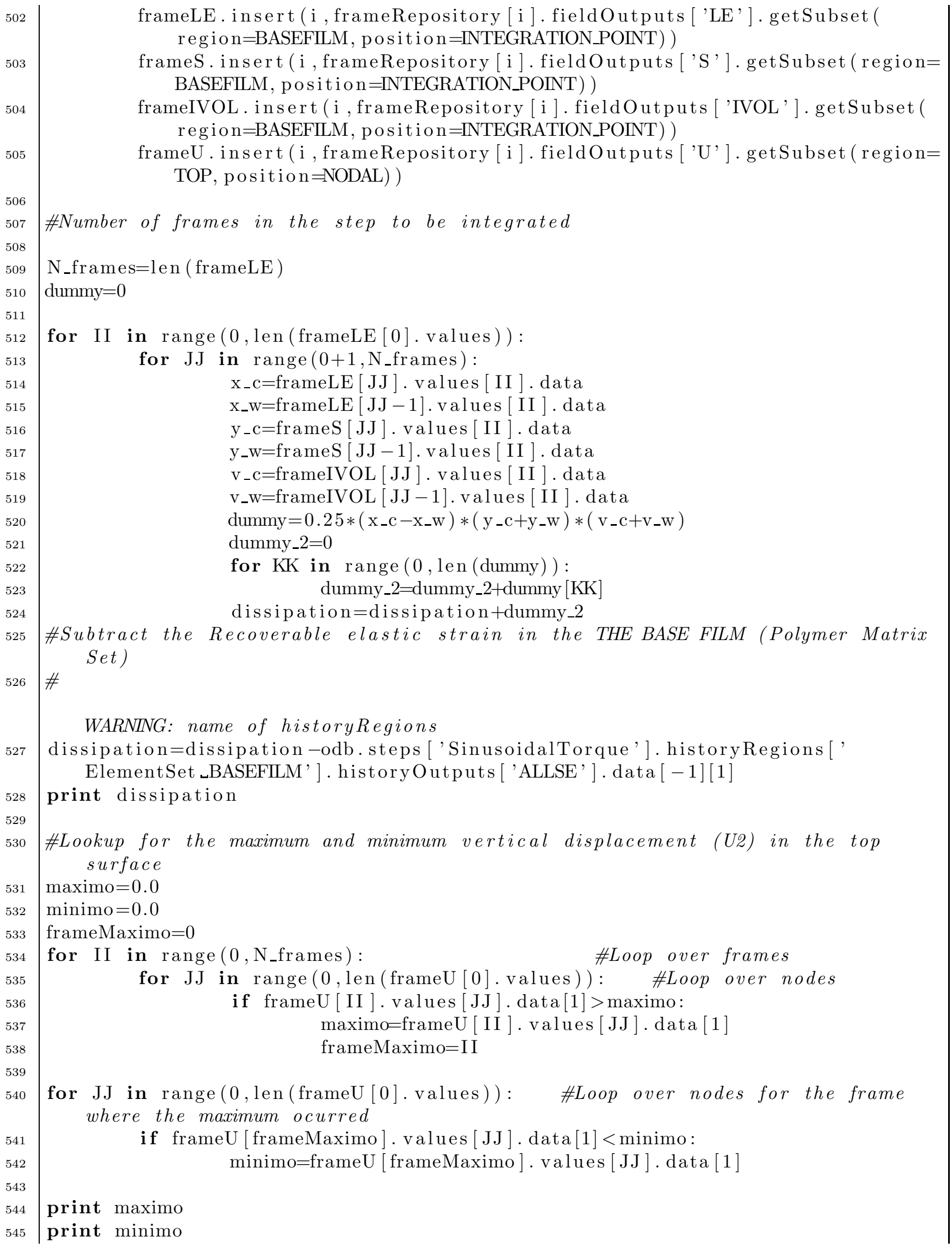




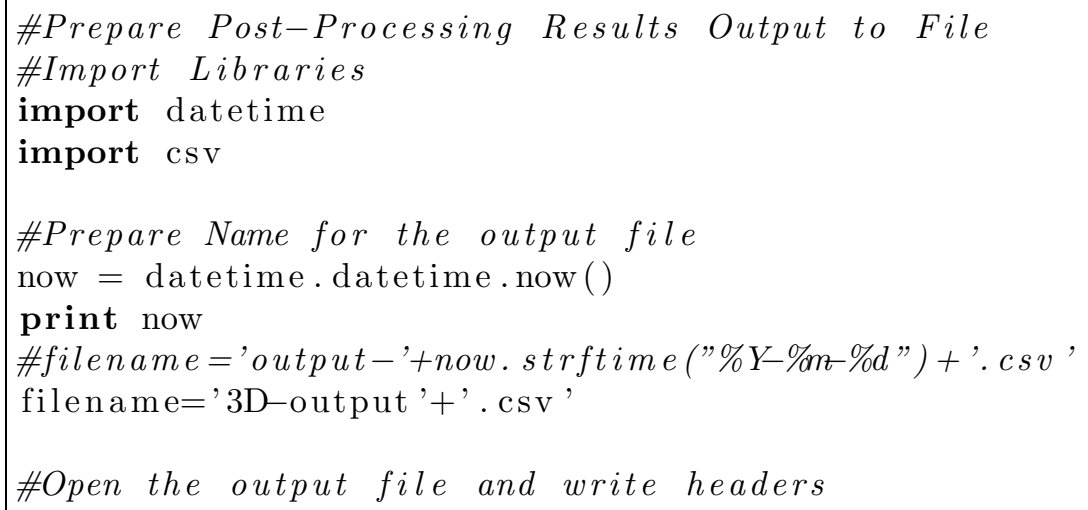


593 fOut.close () 


\section{Appendix F}

\section{Code of Volume Fraction Calculation Script}

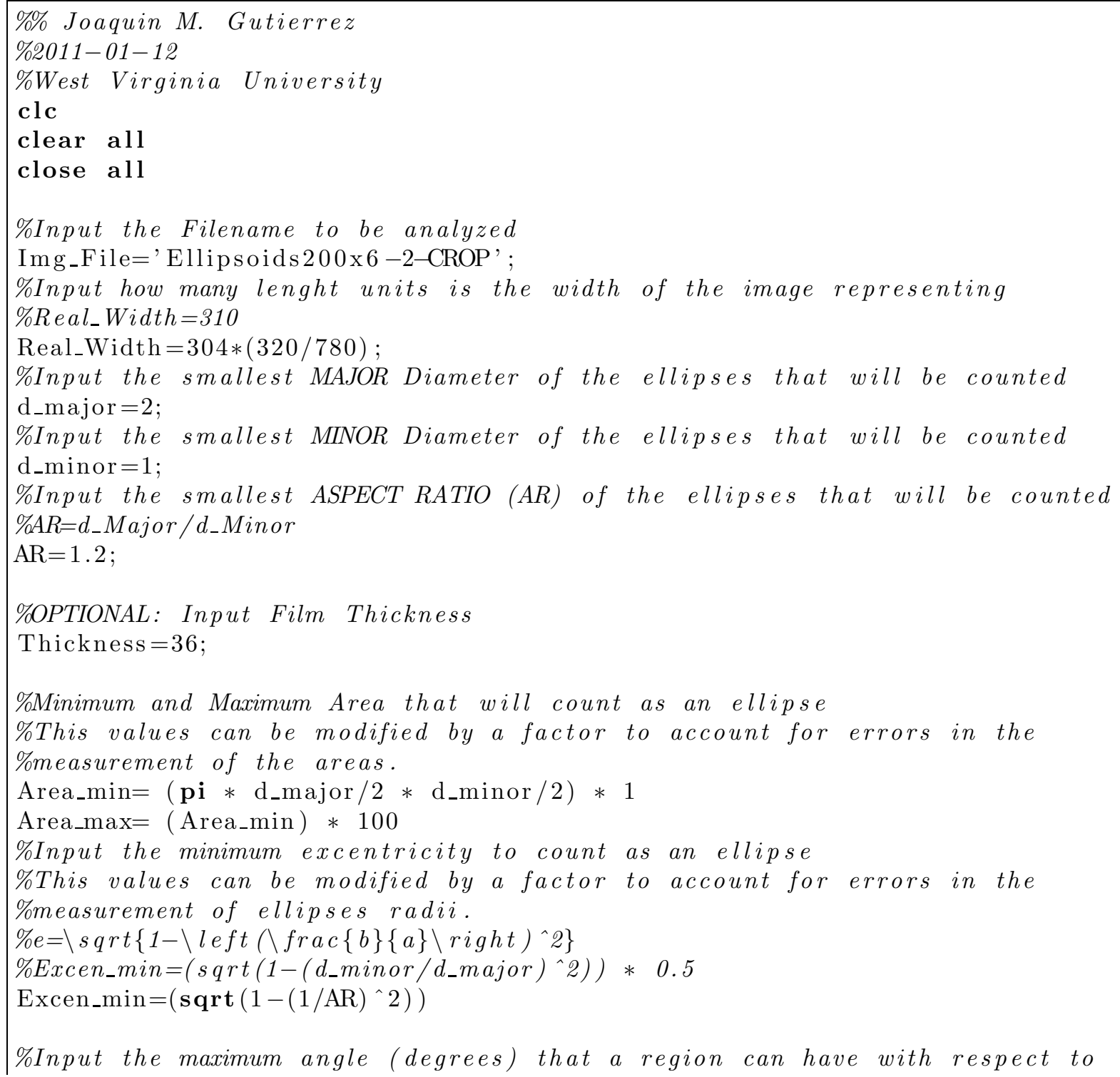




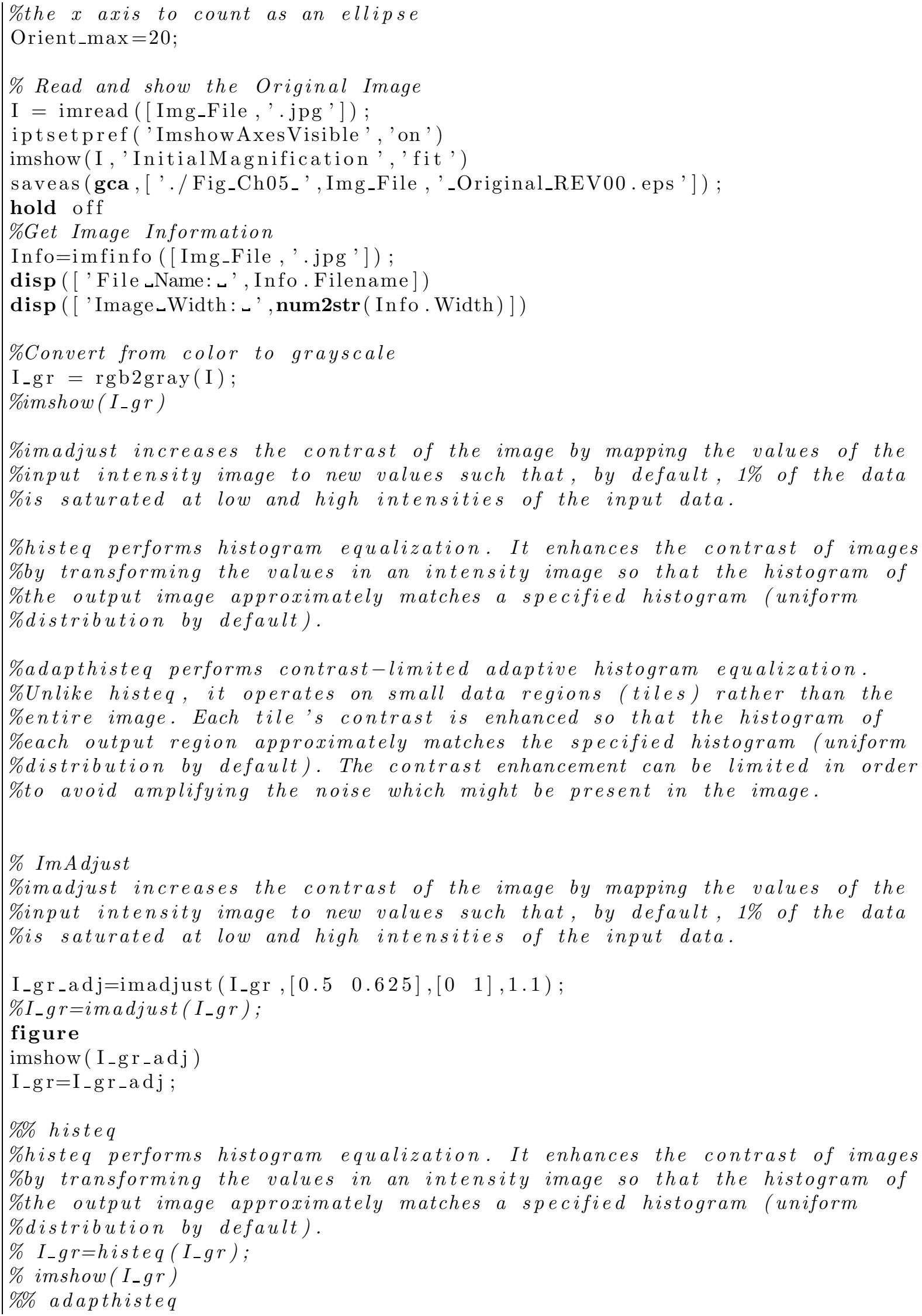




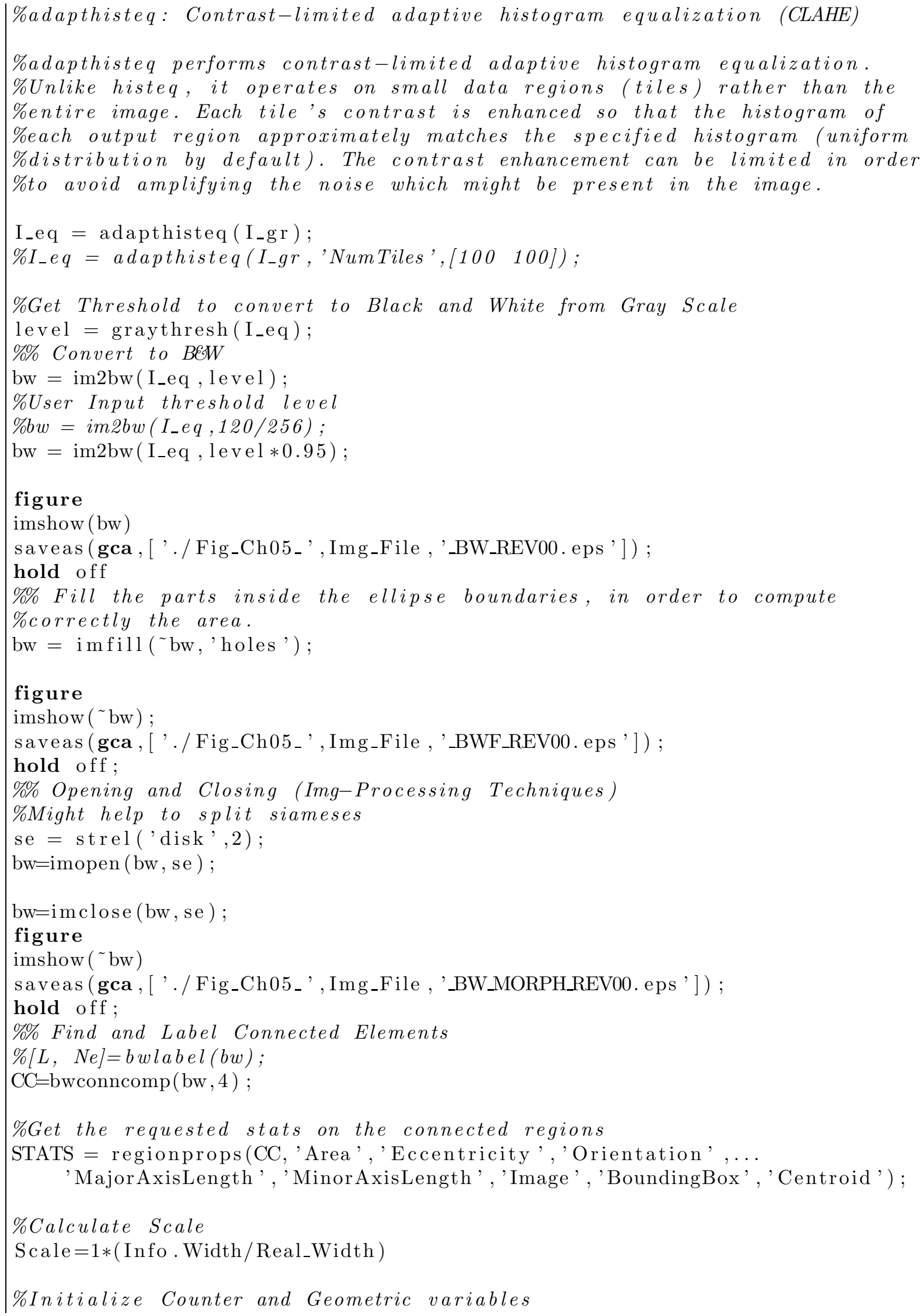


ell_num $=0$;

Areas $=0$

Axes_Major $=0$;

Axes_Minor $=0$;

Orientations $=0$;

$\%$ Create an empty image

bw_filtered=ones (Info. Height, Info. Width);

$\%$ bw_filtered_overlay=rgb2gray (I);

\%oop over all the connected regions found and filter by area an

\%eccentricity (the filter criteria should be changed as needed)

for $\mathrm{n}=1$ : $\mathbf{s i z e}($ STATS, 1$)$

if $\left(\operatorname{STATS}(\mathrm{n})\right.$. Area $>$ Area_min $\left.*\left(\mathrm{Scale}^{\wedge} 2\right)\right) \quad \& \& \ldots$

$\left(\operatorname{STATS}(\mathrm{n})\right.$. Area $<$ Area_max $\left.*\left(\mathrm{Scale}^{\wedge} 2\right)\right) \quad \& \& \ldots$

(STATS $(n)$. Eccentricity $>$ Excen_min) \&\&...

$(\operatorname{abs}(\operatorname{STATS}(\mathrm{n})$. Orientation $)<$ Orient_max $)$

$\% \quad \operatorname{disp}(------------------------------')$

disp (['Region ID: ', num2str(n)])

disp (['Area: ', num2str(STATS(n).Area)])

disp (['MajorAxisLength: ', num2str(STATS(n). MajorAxisLength)])

disp (['MinorAxisLength: ', num2str(STATS(n). MinorAxisLength)])

disp (['Eccentricity: ', num2str(STATS(n). Eccentricity)])

disp (['Orientation: ', num2str(STATS(n). Orientation)])

hold all

\%Show Bounding Box

rectangle ('Position ',STATS(n).BoundingBox, 'LineStyle ', '- ', ...

'EdgeColor ', 'g', , LineWidth ', 1);

\%how the filled ellipse

imshow( 'STATS(n). Image, 'Xdata', [0,STATS(n). BoundingBox(1)], 'Ydata ', [0 , STATS(n). BoundingBox (2)]);

$\%$ Write the filtered images to the file

b w_filtered (round (STATS(n) . BoundingBox (2)) : ...

round $(\operatorname{STATS}(\mathrm{n})$. BoundingBox $(2))+\operatorname{STATS}(\mathrm{n})$. BoundingBox $(4)-1, \ldots$

round $(\operatorname{STATS}(\mathrm{n})$. BoundingBox $(1)): \ldots$

round $(\operatorname{STATS}(\mathrm{n})$. BoundingBox $(1))+\operatorname{STATS}(\mathrm{n})$. BoundingBox $(3)-1)=\ldots$ STATS $(n)$. Image;

\% Write the filtered images on top of the original image

$b w_{-}$filtered_overlay (round(STATS(n). BoundingBox(2)) :... round (STATS (n). BoundingBox (2)) +STATS(n). BoundingBox (4) - 1,... round $(S T A T S(n)$. BoundingBox (1)) : . . . round $(\operatorname{STATS}(n)$. BoundingBox (1) ) $+\operatorname{STATS}(n)$. BoundingBox (3) -1$)=\ldots$ STATS $(n)$. Image;

\%Increase Counter by 1

ell_num=ell_num +1 ;

\%Save the coordinates of the centroids, Area and axes for the \%ellipses within the criteria

Centroids (ell_num , : $=\operatorname{STATS}(\mathrm{n})$. Centroid;

Areas (ell_num $)=\operatorname{STATS}(\mathrm{n})$. Area;

Axes_Major ( ell_num )=STATS(n) . Major AxisLength ;

Axes_Minor (ell_num )=STATS(n) . Minor AxisLength ;

Orientations (ell_num) $=\operatorname{STATS}(\mathrm{n})$. Orientation;

end

end

\%Create new figure to show only the regions that passed the filter

$\%$ figure 


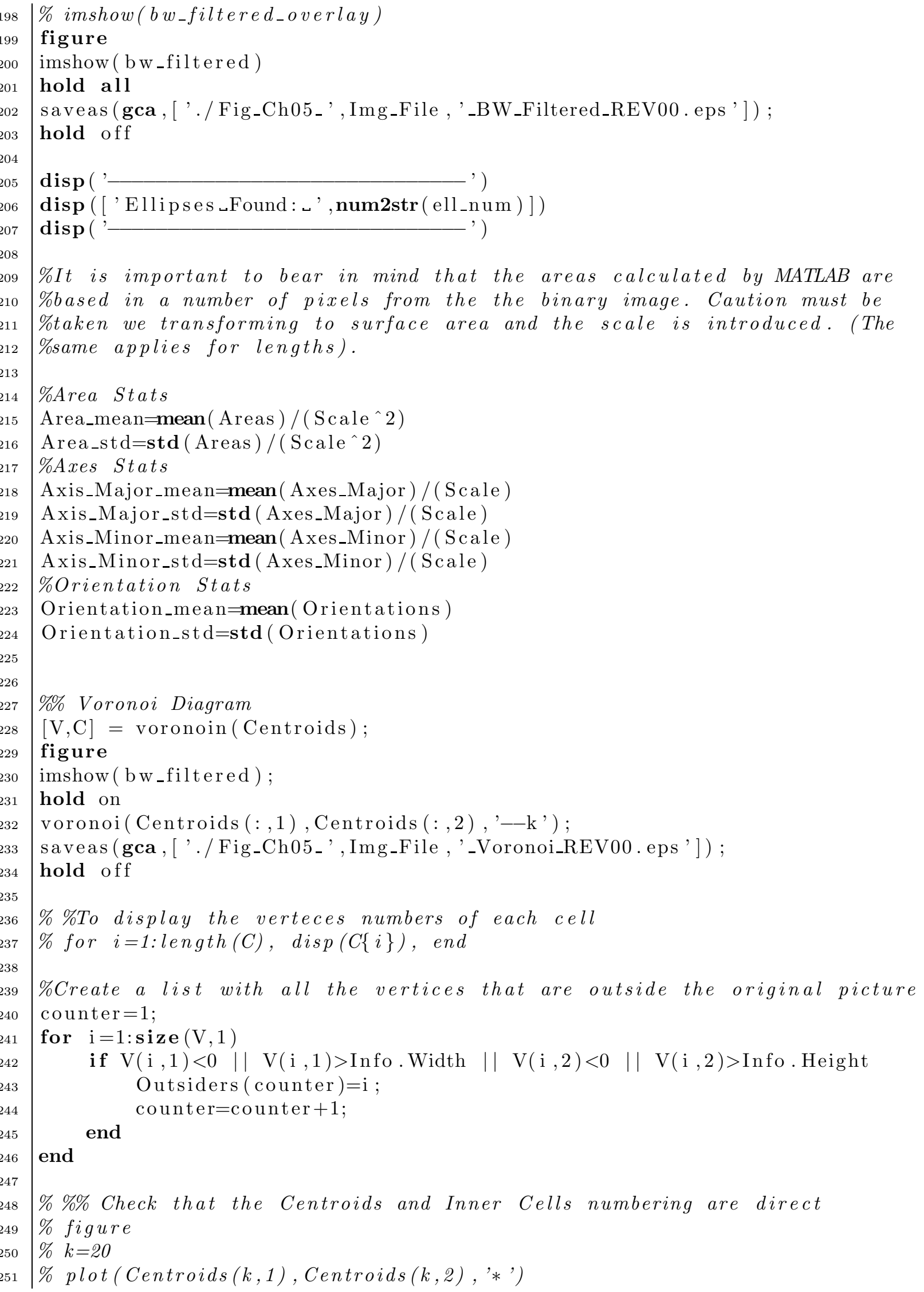




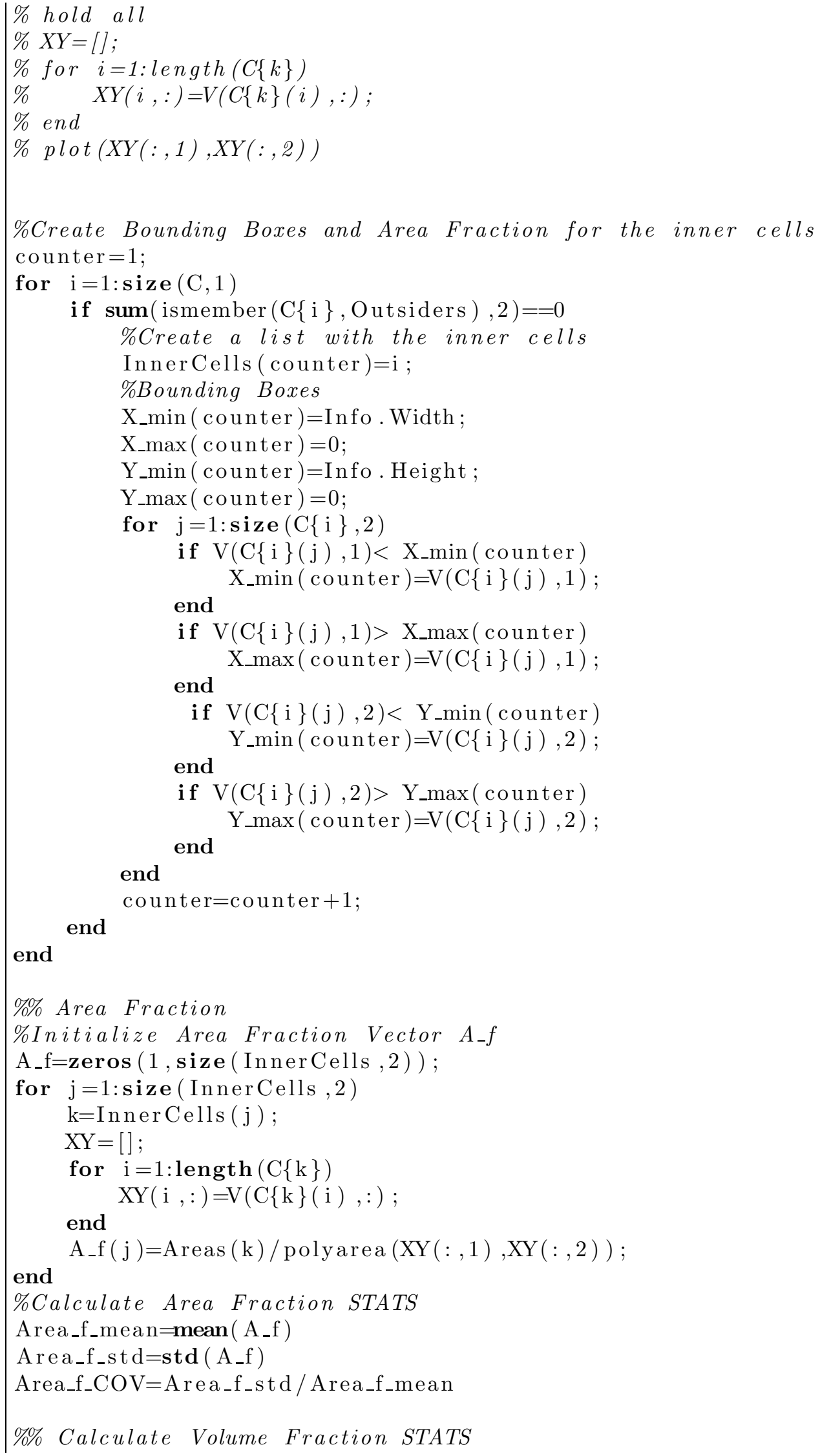




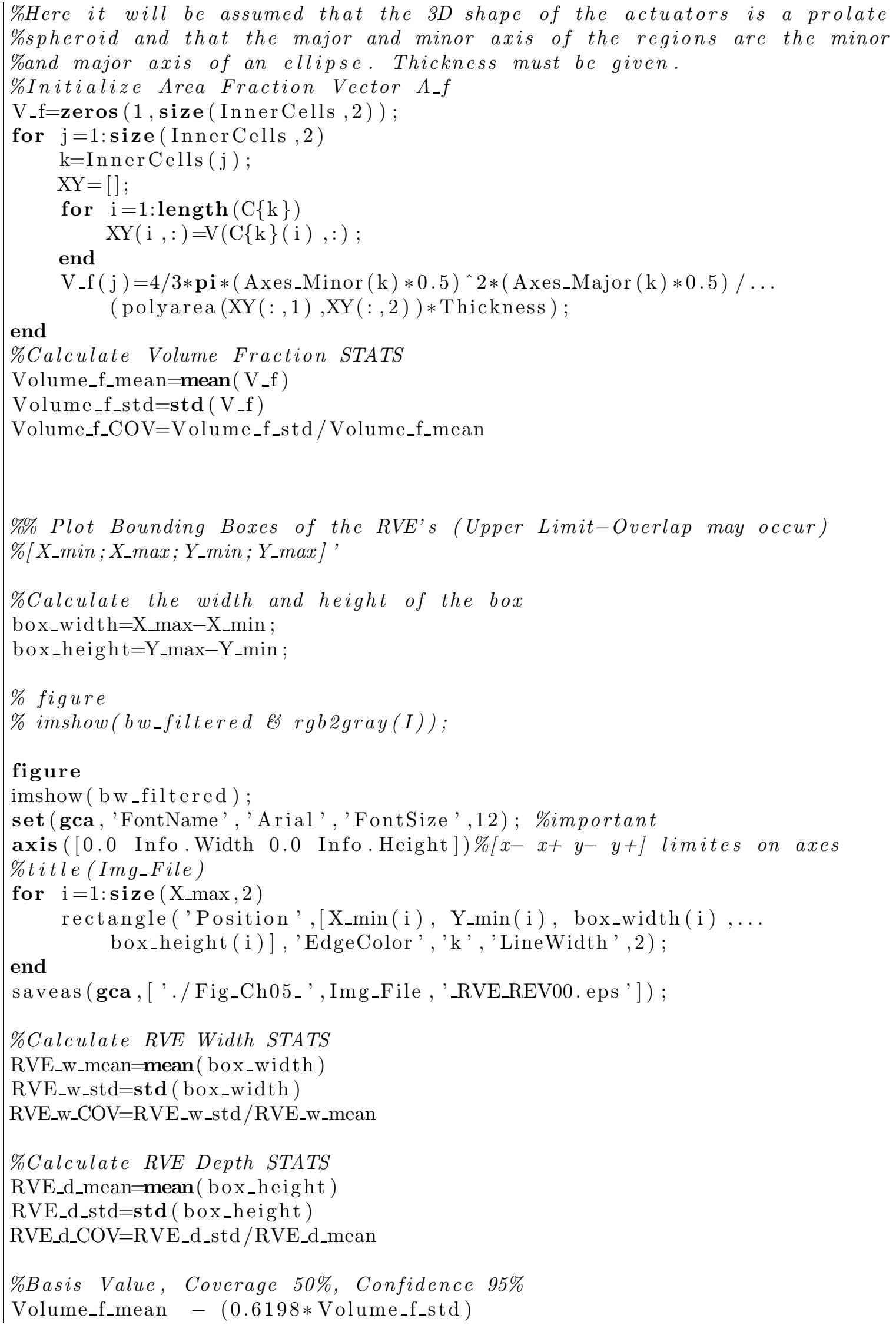




\section{Appendix G}

\section{Companion CD Contents}

\begin{tabular}{|l|l|l|}
\hline Folder & File Name & Description \\
\hline Fit & fit10.m & MATLAB program listed in Appendix A.1 \\
\hline Fit & bb10.m & MATLAB program listed in Appendix A.2 \\
\hline Fit & bb10_error.m & MATLAB program listed in Appendix A.3 \\
\hline Fit & fminsearchcon.m & MATLAB Add-on Minimization Program (by J. D'Errico) \\
\hline AppxB & dissipation.py & Python Script for the dissipation calculation (Appendix B.3) \\
\hline AppxE & CONTACT02c.py & Python Script for ABAQUS - Contact Model (Appendix E.1) \\
\hline AppxE & VISCO17.py & Python Script for ABAQUS - 2D Model (Appendix E.2) \\
\hline AppxE & 3DMODEL05d.py & Python Script for ABAQUS - 3D Model (Appendix E.3) \\
\hline Ch05-REV01/Image A & EC-REV09-1-200x-CROP-B.m & MATLAB program used for the image processing of Image A \\
\hline Ch05-REV01/Image A & 1-200x-CROP-B.jpg & Image A \\
\hline Ch05-REV01/Image B & EC_REV09_6_2_CROP.m & MATLAB program used for the image processing of Image B \\
\hline Ch05-REV01/Image B & Ellipsoids200x6-2-CROP.jpg & Image B \\
\hline
\end{tabular}




\section{Bibliography}

[1] Analysis: Defect 97792. Technical report, ANSYS, 2010.

[2] Helmut Alt, David Hsu, and Jack Snoeyink. Computing the largest inscribed isothetic rectangle. In Proceedings of the Seventh Canadian Conference on Computational Geometry, 1995.

[3] Pedro Areias and Karel Matous. Finite element formulation for modeling nonlinear viscoelastic elastomers. Computer Methods in Applied Mechanics and Engineering, 197(5152):4702 - 4717, 2008.

[4] Ellen M. Arruda and Mary C. Boyce. A three-dimensional constitutive model for the large stretch behavior of rubber elastic materials. Journal of the Mechanics and Physics of Solids, 41(2):389 - 412, 1993.

[5] ASTM. D575-91 Standard Test Methods for Rubber Properties in Compression.

[6] ASTM. D638-10 Standard Method for Tensile Properties of Plastics.

[7] Ever J. Barbero. Finite Element Analysis of Composite Materials. CRC Press, 2007.

[8] Ever J. Barbero. Creep and Fatigue in Polymer Matrix Composites, chapter Timetemperature-age superposition principle for predicting long-term response of linear viscoelastic materials, pages 48-69. Woodhead Publishing, 2010.

[9] M. Berg, O. Cheong, M. Kreveld, and M. Overmars. Computational geometry: algorithms and applications. Springer, third edition, 2008.

[10] Jorgen Bergstrom. http://polymerfem.com.

[11] J.S. Bergstrom. Large Strain Time-Dependent Behavior of Elastomeric Materials. PhD thesis, MIT, 1999.

[12] J.S. Bergstrom. Determination of material parameters for the 8-chain model for use with abaqus, ls-dyna and ansys. Technical report, http://polymerfem.com/, 2002.

[13] J.S. Bergstrom and M.C. Boyce. Constitutive modeling of the large strain timedependent behavior of elastomers. Journal of the Mechanics and Physics of Solids, 46(5):931-954, MAY 1998. 
[14] J.S. Bergstrom and M.C. Boyce. Mechanical behavior of particle filled elastomers. Rubber Chemistry and Technology, 72(4):633-656, SEP-OCT 1999.

[15] J.S. Bergstrom and M.C. Boyce. Large strain time-dependent behavior of filled elastomers. Mechanics of Materials, 32(11):627-644, NOV 2000.

[16] J.S. Bergstrom and M.C. Boyce. Constitutive modeling of the time-dependent and cyclic loading of elastomers and application to soft biological tissues. Mechanics of Materials, 33(9):523-530, SEP 2001.

[17] Allan F. Bower. Applied Mechanics of Solids. CRC Press, 2009.

[18] D. Braess. Finite elements: theory, fast solvers, and applications in elasticity theory. Cambridge University Press, 2007.

[19] Computer Aided Material Preselection by Uniform Standards. http://www . campusplastics.com/.

[20] Darran R. Cairns. Personal communication, 2009-2010.

[21] Darran R. Cairns, Matthew S. Shafran, Konstantinos A. Sierros, Wade W. Huebsch, and Aaron J. Kessman. Stimulus-responsive fluidic dispersions of rod shaped liquid crystal polymer colloids. Materials Letters, 64(10):1133 - 1136, 2010.

[22] Darran R. Cairns, Merwin Sibulki, and Gregory P. Crawforda. Switching dynamics of suspended mesogenic polymer microspheres. Applied Physics Letters, 78(18):2643-2645, 2001.

[23] Husnu Dal. Computational aspects of Bergstrom-Boyce finite viscoelasticity model. In Constitutive models for rubber, editor, Constitutive models for rubber, volume 5. Balkema, 2008.

[24] Husnu Dal and Michael Kaliske. Bergstrom-Boyce model for nonlinear finite rubber viscoelasticity: theoretical aspects and algorithmic treatment for the FE method. Computational Mechanics, 44(6):809-823, November 2009.

[25] Dassault Systèmes. ABAQUS Analysis User's Manual (6.10).

[26] Dassault Systèmes. ABAQUS Theory Manual (6.9).

[27] John D'Errico. http://www.mathworks.com/matlabcentral/fileexchange/ 13469-fminsearchcon, December 2006.

[28] P. D. Gall, W. W. Huebsch, S. D. Hamburg, and A.P. Rothmayer. Dynamic roughness as a means of leading edge separation flow control. Submitted, 2010.

[29] R.C. González, R.E. Woods, and S.L. Eddins. Digital Image processing using MATLAB. Pearson Prentice Hall, 2004. 
[30] J. Hildebrandt, H. Fukaya, and C. J. Martin. Simple uniaxial and uniform biaxial deformation of nearly isotropic incompressible tissues. Biophysical Journal, 9:781791, 1969.

[31] Gerhard A. Holzapfel. Nonlinear Solid Mechanics: A Continuum Approach for Engineering. Wiley, John \& Sons, Incorporated, 2000.

[32] Wade W. Huebsch. Personal communication, 2010.

[33] W.W. Huebsch. Dynamic surface roughness for aerodynamic flow control. In 42nd AIAA Aerospace Sciences Meeting and Exhibit, 2004.

[34] Sheldon (ANSYS) Imaoka and West Virginia University Joaquin M. Gutierrez. Personal communication, November 2009.

[35] Johansson and Runesson. Calibration of the Bergstrom-Boyce model. Research Report 2005:02, Department of Applied Mechanics Division Material \& Computational Mechanics, Chalmers University of Technology, 2005.

[36] Johansson and Runesson. Calibration of a class of non-linear viscoelasticity models with sensitivity assessment based on duality. Int. J. Numer. Meth. Engng., 69(12):2513-2537, 2007.

[37] Goy Teck Lim. Scratch Behavior of Polymers. PhD thesis, Texas A\&M University, 2005.

[38] G.T. Mase, R.E. Smelser, and G.E. Mase. Continuum Mechanics for Engineers. CRC series in computational mechanics and applied analysis. CRC Press, 2009.

[39] B.D. Matheis, W.W. Huebsch, and A.P. Rothmayer. Separation and unsteady vortex shedding from leading edge surface roughness. Technical report, Iowa State University, 2004.

[40] MATLAB. Natick, Massachusetts: The MathWorks Inc.

[41] N. J. Morris. Personal communication, 2010-2011.

[42] N. J. Morris, K. A. Sierros, D. R. Cairns, and S. N. Kukureka. Polymer dispersed liquid crystal films with electrically switchable roughness. (Submitted).

[43] M. Orlowski. A new algorithm for the largest empty rectangle problem. Algorithmica, 5:65-73, 1990. 10.1007/BF01840377.

[44] Matthew S. Shafran. Responsive liquid crystal polymer rods. Master's thesis, College of Engineering and Mineral Resources at West Virginia University, 2008.

[45] L.R.G. Treloar. Physics of Rubber Elasticity (3rd Edition). Oxford University Press, 2009. 
[46] I.M. Ward and J. Sweeney. An introduction to the mechanical properties of solid polymers. Wiley, 2004.

[47] K. Washizu. Variational methods in elasticity and plasticity. International series of monographs on aeronautics and astronautics: Solid and structural mechanics. Pergamon Press, 1974.

[48] Wikipedia. Polyvinyl alcohol. http://en.wikipedia.org/w/index.php? title=Polyvinyl_alcohol\&oldid=396893114, 2010. [Online; accessed 3-January2011].

[49] O.C. Zienkiewicz and R.L. Taylor. The finite element method for solid and structural mechanics. The Finite Element Method. Elsevier Butterworth-Heinemann, 2005.

[50] O.C. Zienkiewicz, R.L. Taylor, and J.Z. Zhu. The finite element method: its basis and fundamentals. The Finite Element Method. Elsevier Butterworth-Heinemann, 2005.

John $\mathrm{H}$. Dasaly Hagen 